\title{
INFERÊNCIA EM MODELOS COM ERROS NAS VARIÁVEIS ATRAVÉS DO MÉTODO DO ESCORE CORRIGIDO
}

\author{
Patricia Cristina Gimenez
}

TESE APRESENTADA

$\mathrm{AO}$

INSTITUTO DE MATEMÁTICA E ESTTATÍSTICA

DA

UNIVERSIDADE DE SÃO PAULO

PARA

OBTENÇÃO DO GRAU

DE

DOUTOR EM ESTATÍSTICA

\author{
Área de Concentração: Estatística \\ Orientador: Prof. Dr. Heleno Bolfarine
}

- São Paulo, julho de 1997 - 


\title{
INFERÊNCIA EM MODELOS COM ERROS NAS VARIÁVEIS ATRAVÉS DO MÉTODO DO ESCORE CORRIGIDO
}

\author{
Este exemplar corresponde à redação final \\ da tese devidamente corrigida e \\ defendida por Patricia Cristina Gimenez \\ e aprovada pela comissão julgadora.
}

São Paulo, 25 de julho de 1997.

Banca examinadora:

- Prof ‥ Drํ․ Heleno Bolfarine (Orientador) - IME - USP

- Profo. Dro. Antonio Carlos Pedroso de Lima - IME - USP

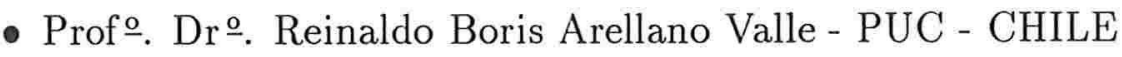

- Prof o. Dr‥ Enrico Colosimo - UFMG

- Profo. Dr ํ. Mauro Sérgio de Freitas Marques -IMECC - UNICAMP 


\section{Agradecimentos}

Ao Prof. Dr. Heleno Bolfarine, pela confiança em mim depositada, pela orientação segura e experiência transmitida durante o desenvolvimento deste trabalho.

Ao Prof. Dr. Enrico Colosimo, pelas valiosas sugestões e discussões em grande parte deste trabalho e por seu indispensável apoio computacional.

Ao IME-USP, por ter me acolhido como mais um de seus estudantes estrangeiros.

À Universidad Nacional de Mar del Plata, por ter me liberado o tempo necessário para a realização do doutorado, e aos meus colegas do Departamento de Matemática da Faculdad de Ciencias Exactas y Naturales, pelo apoio. Muito especialmente, agradeço ao Pedro e à Sonia.

Aos meus colegas do Doutorado em Estatística, em especial à Loretta, ao Vicente, e aos meus compatriotas Viviana, Verônica, Jesús e Carolina, pelo companheirismo e apoio.

Aos meus amigos Sonia e Marcelo, com quem compartilhei todos os momentos de minha vida universitária, pelo carinho e incentivo.

Ao meu irmão José Luis, pelo carinho, estímulo e sobretudo pelos conselhos inteligentes às minhas preocupações.

Ao Cristian, por tanta paciência, compreensão e carinho que tornaram muito feliz este período de minha vida.

Ao Walter, pela paciência e eficiência na digitação desta tese.

À CAPES e à UNMDP, pelo apoio financeiro.

Finalmente, aos meus pais, pelo amor e constante apoio, e porque mais que ninguém torceram e me acompanharam nesta etapa de minha formação. A eles dedico este trabalho. 


\section{Abstract}

In this thesis, we present a general formulation of the corrected score method (Nakamura, 1990) for inference in measurement error models, which encompasses both linear and nonlinear models, functional and structural cases. We present a simple model for which a corrected score function cannot be obtained through differentiating a corrected loglikelihood although it exists. Thus, regularity conditions with no reference to log-likelihood are given under which the corrected score function yields consistent and asymptotically normal estimators. Hypothesis tests based on corrected score estimators are proposed. The asymptotic relative efficiency of these tests to the naive tests and the tests based upon the true covariates is derived, for a particular generalized linear measurement error model class. Applications made to comparative calibration and Weibull regression models yields interesting results. 


\section{Índice}

1 Introdução 1

1.1 Formulação do probler $\ldots \ldots \ldots \ldots \ldots \ldots$

1.2 Objetivos do trabalho $\ldots \ldots \ldots \ldots \ldots \ldots$

1.3 Resumo de conteúdos .................. 6

2 O método do escore co 10

2.1 Introdução . . . . . . . . . . . . . . . . . . 10

2.2 Descrição do método f. . . . . . . . . . . . . . . 13

2.3 Propriedades assintó $\quad$ os estimadores. . . . . . . . . . . . 20

2.3 .1 Condições de $\quad$ ridade . . . . . . . . . . . . . . . . . . 20

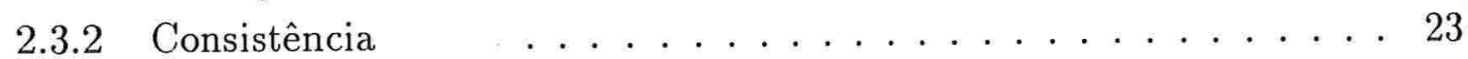

2.3.3 Normalidade ótica . . . . . . . . . . . . . 26

2.3.4 Estimação d iz de covariâncias assintótica . . . . . . . . 28

2.4 Estimação quando : iz de covariâncias do erro é desconhecida . . . . . 31

2.5 Existência e unicidi escore corrigido . . . . . . . . . . . 34

2.6 Exemplos ... . . . . . . . . . . . . 36

2.7 Extensão do métolo ındo a distribuição do erro de medição é normal contaminada . . . . . . . . . . . . . . . . . . . 39

3 Testes de hipóteses $\quad 42$

3.1 Introdução . . . . . . . . . . . . . . . . . . . . . . 42

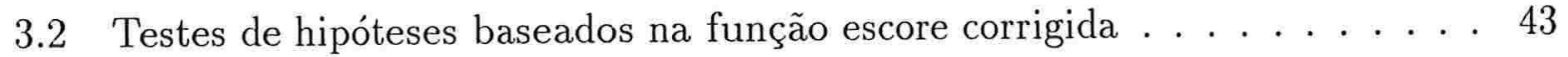

3.2 .1 Um estudo de simulação . . . . . . . . . . . . . . . . . . . 50

3.3 Comparação de testes escore . . . . . . . . . . . . . . . . 53

3.3.1 Testes escore eficiente e "naive" . . . . . . . . . . . . . 55

3.3 .2 Teste escore corrigido . . . . . . . . . . . . . . . . . . . . 67 67

3.4 Comparação de testes de Wald . . . . . . . . . . . . . . . . . . . 69

3.4 .1 Um estudo de simulação . . . . . . . . . . . . . . . . 77 
4 O modelo de calibração comparativa $\quad 79$

4.1 Introdução . . . . . . . . . . . . . . . . . . . . 79

$4.2 \mathrm{O}$ caso $\sigma_{u}^{2}$ conhecida . . . . . . . . . . . . . . . 81

4.2.1 O estimador do escore corrigido, propriedades assintóticas . . . . . 82

$4.2 .2 \mathrm{O}$ modelo de regressão linear simples . . . . . . . . . . . . 86

$4.3 \mathrm{O}$ caso $\lambda_{k}=\sigma_{k}^{2} / \sigma_{u}^{2}, k=1, \ldots, p$, conhecidas . . . . . . . . . . 89

4.3.1 O estimador do escore corrigido, propriedades assintóticas . . . . 89

4.3 .2 O modelo de regressão linear simples . . . . . . . . . . . . . . . . . 93

4.3.3 Comparação de estimadores . . . . . . . . . . . . . . . . 95

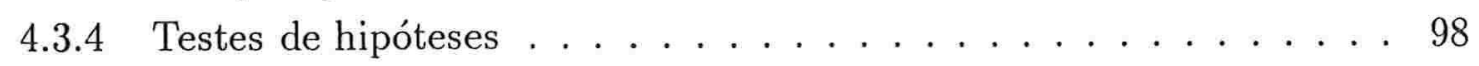

4.3 .5 Ilustração numérica . . . . . . . . . . . . . . . . . . 101

5 O modelo de regressão Weibull 104

5.1 Introdução . . . . . . . . . . . . . . . . . . . . . . . 104

5.2 Estimação . . . . . . . . . . . . . . . . . . . 105

5.2 .1 O estimador do escore corrigido . . . . . . . . . . . 107

5.2 .2 Alguns estimadores propostos na literatura . . . . . . . . . . 108

5.2 .3 Novo estimador . . . . . . . . . . . . . . . . . . . 111

5.2 .4 Um estudo de simulação . . . . . . . . . . . . . . . . . . . . . 112

5.3 Testes de hipóteses . . . . . . . . . . . . . . . . . . . . . . . . . . . . . . . . . . . . . . . . . . . . .

5.3 .1 Testes escore . . . . . . . . . . . . . . . . 122

5.3 .2 Testes de Wald . . . . . . . . . . . . . . . . . . 124

$\begin{array}{ll}\text { Considerações finais } & 127\end{array}$

$\begin{array}{ll}\text { Referências bibliográficas } & 130\end{array}$ 


\section{Capítulo 1}

\section{Introdução}

\subsection{Formulação do problema}

Na literatura estatística, o termo modelo de regressão com erros nas variáveis refere-se a um modelo para uma resposta $y$, em termos de preditores ou covariáveis $z$ que não podem ser medidas exatamente. No lugar do verdadeiro valor da variável preditora, observa-se um estimador $x$ deste valor. Os parâmetros no modelo relacionando $y$ e $z$ não podem ser estimados diretamente ajustando $y$ a $x$, uma vez que os dados observados não correspondem ao modelo postulado.

A maioria das medições físicas em diferentes áreas da ciência tem erro. Um bom exemplo de preditor medido com erro é a pressão sangüínea sistólica, cujo valor verdadeiro difere do observado numa visita médica, devido à variabilidade introduzida pelo operador, hora do dia e outros fatores como estresse do paciente (veja por exemplo, o famoso estudo prospectivo de doenças cardiovasculares de Framingham, Kannel et al., 1986). Outros exemplos incluem quantidades de radiação recebida pelos sobreviventes das bombas atômicas lançadas sobre o Japão (Okajima et al., 1985) e, em geral, as avaliações feitas através de questionários.

Dado que os erros de medição são um fato intrínseco na maioria dos problemas e que, embora trate-se de melhorar a qualidade das medições, uma completa prevenção resulta 
impossível, torna-se necessário então, levar em conta a presença dos erros na análise estatística.

É sabido que, quando o interesse é a relação entre a resposta e as verdadeiras covariáveis, ignorar o erro de medição pode causar estimadores inconsistentes dos parâmetros do modelo (Stefanski, 1985) e, muitas vezes o erro pode viciar seriamente os estimadores. O estimador obtido por algum procedimento usual (mínimos quadrados, máxima verossimilhança, etc.), quando simplesmente as verdadeiras covariáveis não observadas $z$ são substituídas pelas covariáveis observadas, é chamado estimador "naive".

Um dos objetivos da análise de modelos com erros nas variáveis é encontrar estimadores consistentes ou "aproximadamente consistentes". Como exemplo, considere o interesse da epidemiologia na atualidade em obter relações quantitativas entre fatores de risco e doença, priorizando como conseqüência a obtenção de estimadores não viciados. O efeito do erro de medição em testes de hipóteses é outro assunto de interesse.

Um fato a ser levado em conta na análise estatística na presença de erros de medição é o modelo assumido para o erro. O modelo clássico de erros de medição tem sido o mais considerado na literatura, e será assumido ao longo deste trabalho. Se $z$ é o verdadeiro valor do preditor e $x$ é o valor observado, modelamos $x$ como função de $z$. De alguma forma, isto significa considerar que $z$ causa $x$. Por exemplo, $z$ poderia ser o consumo real de cigarros e $x$ o consumo reportado. Neste trabalho consideramos uma estrutura aditiva, assumindo que $x$ é igual a $z$ mais um erro de medição. Outra suposição que será adotada é que, uma vez que o valor de $z$ for observado, nenhuma informação adicional sobre $y$ é obtida observando $x$ (modelo não diferencial). Além disso, num modelo clássico de erros de medição podemos considerar dois submodelos, o modelo funcional, onde assumimos que os verdadeiros valores das covariáveis $z$ são constantes fixas, desconhecidas, e o modelo estrutural quando $z$ é considerada uma variável aleatória com alguma distribuição. Outros modelos e estruturas de erros de medição têm sido estudados na literatura (veja, por 
exemplo, Berkson, 1950, e Carroll et al., 1995).

Existe uma extensa literatura sobre modelos com erros nas variáveis em regressão linear (veja Fuller, 1987, e referências ali citadas). Desde começos dos anos 80, com a publicação dos trabalhos de Prentice (1982), em análise de sobrevivência, Stefanski e Carroll (1985), em regressão logística, e Armstrong (1985), em modelos lineares generalizados, entre outros, tem havido um interesse crescente no estudo de modelos não lineares. A obtenção de estimadores consistentes dos parâmetros desconhecidos em modelos com erros nas variáveis não lineares resulta complicada, mesmo quando o erro de medição é assumido normalmente distribuído, com variância conhecida. A dificuldade é devida ao fato de que as verdadeiras covariáveis desconhecidas devem ser removidas da verossimilhança, seja por integração no caso de um modelo estrutural, seja incluindo-as no espaço paramétrico, no caso de um modelo funcional. Em ambas as situações, os cálculos podem ser bastante complicados, como mencionado nos trabalhos de Stefanski (1985), Wolter e Fuller (1982), Carroll et al. (1984), Schafer (1987) e Whittemore e Keller (1988), dentre outros.

Existe uma grande variedade de técnicas que produzem estimadores "aproximadamen-

te consistentes" em modelos não lineares, fazendo correções explícitas para o vício dos estimadores ou propondo estimadores alternativos sob certas suposições. Vários métodos propostos são apropriados somente para estimação no modelo estrutural, desde que requerem conhecimento da distribuição condicional de $z$ dado $x$. Entre eles podemos citar as aproximações ao estimador de máxima verossimilhança, obtidas por Whittemore e Keller (1988) e Armstrong (1985), e mais recentemente o método de regressão-calibração (Carroll e Stefanski, 1990), que propõe substituir as verdadeiras covariáveis pela esperança condicional de $z$ dado $x$, antes de realizar uma análise usual. Outros métodos exploram a estrutura particular de determinados modelos e produzem estimadores consistentes sem necessidade de suposições sobre a distribuição condicional de $z$ dado $x$. Nesta direção, podemos mencionar o trabalho de Stefanski e Carroll (1987) para modelos lineares gene- 
ralizados, sob uma estrutura aditiva de erros de medição, normalmente distribuídos. Por outro lado, Liang e Liu (1991) sugerem um enfoque baseado em equações de estimação para encontrar estimadores consistentes, diretamente. Estimadores corrigidos são obtidos por Stefanski (1985), baseado em expansões de Taylor do vício assintótico do estimador "naive". Estes estimadores podem ser facilmente obtidos sem suposições sobre a distribuição das verdadeiras covariáveis, somente a estrutura do erro de medição (aditiva ou multiplicativa) é requerida. Na "Statistics in Medicine", v.8, (1989), são desenvolvidos vários métodos aplicados a estudos reais.

Nakamura (1990), introduziu uma técnica de estimação baseada na idéia de função escore corrigida. Não são necessárias suposições sobre a distribuição das verdadeiras covariáveis, que podem ser aleatórias como num modelo estrutural ou constantes fixas desconhecidas como num modelo funcional. O método requer uma estrutura aditiva de erros de medição, normalmente distribuídos. Além disso, assume-se que a matriz de covariâncias associada ao erro é conhecida, ou um estimador da mesma é disponível. A função escore corrigida depende somente das covariáveis observadas e dos parâmetros estruturais do modelo. É definida como uma função cuja esperança com respeito à distribuição do erro de medição coincide com a função escore usual baseada nas verdadeiras covariáveis desconhecidas. Nakamura assume também que esta função corresponde ao gradiente de uma log-verossimilhança corrigida. No entanto, um escore corrigido pode ser obtido sem referência a uma log-verossimilhança corrigida. Segue da definição que todo escore corrigido é não viciado, ou seja, tem esperança zero quando avaliado no verdadeiro valor do parâmetro. Sob condições de regularidade apropriadas, esta propriedade implica na existência de estimadores consistentes e assintoticamente normais, dos parâmetros do modelo. Esses resultados são enunciados em Nakamura (1990) baseados na existência de uma log-verossimilhança corrigida, sem apresentar condições de regularidade e provas rigorosas. 
A maior parte da literatura estatística em modelos com erros nas variáveis não lineares concentra-se na avaliação dos efeitos dos erros na estimação. Contudo, os antecedentes dados acima mostram que poucos métodos produzem estimadores consistentes dos parâmetros e permitem o estudo unificado dos casos funcional e estrutural. Sendo a maioria dos estimadores "aproximadamente consistentes", métodos de reamostragem são usados para fazer inferência. A dificuldade na obtenção de resultados assintóticos para serem usados em conjunção com tais estimadores tem concentrado o estudo de testes de hipóteses no comportamento de testes "naive". No contexto de testes de hipóteses, "naive" significa que o erro de medição é ignorado e, simplesmente, o valor não observado $z$ da covariável é substituído pelo valor observado $x$, num teste que é válido quando $z$ é observado. O problema de testes de hipóteses em modelos lineares generalizados é considerado nos trabalhos de Tosteson e Tsiatis (1988) e Stefanski e Carroll (1990).

\subsection{Objetivos do trabalho}

Motivados pelos fatos mencionados acima, o objetivo geral deste trabalho é a inferência estatística baseada no método do escore corrigido, para modelos de regressão com erros nas variáveis. A idéia é apresentar a metodologia baseada na função escore corrigida (Nakamura, 1990), sem referência à existência de uma função de log-verossimilhança corrigida, e tratar de forma unificada os modelos funcional e estrutural. Baseados na consistência e normalidade assintótica dos estimadores, testes de hipóteses podem ser propostos. A aplicação do método é considerada em dois modelos de interesse. Assim, os objetivos específicos desta tese podem ser resumidos como segue:

(1) apresentar condições de regularidade apropriadas e provas de consistência e normalidade assintótica dos estimadores do escore corrigido. Estas condições são propostas sem referência à função de log-verossimilhança corrigida e resultam ser diferentes das estabelecidas para $M$-estimadores. Além disso, permitem a consideração dos 
modelos funcional e estrutural de forma unificada;

(2) propor alguns testes assintóticos tipo razão de verossimilhança, escore e de Wald, baseados na função escore corrigida;

(3) calcular as eficiências relativas assintóticas dos testes propostos com relação a testes "naive", para uma classe particular de modelos lineares generalizados estruturais (a família Tweedie, com média log-linear);

(4) aplicar a metodologia do escore corrigido para inferência no modelo de calibração comparativa funcional e estrutural;

(5) estudar o comportamento do estimador do escore corrigido e outros considerados na literatura para um modelo paramétrico de tempos de vida com dados censurados (o modelo de regresão Weibull, no caso estrutural).

\subsection{Resumo de conteúdos}

Em termos de atingir os objetivos definidos acima, este trabalho é organizado em cinco capítulos.

No Capítulo 2, apresentamos a metodologia baseada na função escore corrigida. Mostramos que a suposição básica de obter um escore corrigido como derivada de uma log-verossimilhança corrigida (Nakamura, 1990) pode falhar num modelo simples, como o modelo de regressão linear com razão de variâncias conhecida. Provamos consistência e normalidade assintótica dos estimadores, sob condições de regularidade apropriadas que permitem tratar de forma unificada os modelos funcional e estrutural. As condições de regularidade propostas estabelecem suposições mais fracas no comportamento da seqüência de parâmetros incidentais no caso do modelo funcional, do que as encontradas na literatura. Além disso, são diferentes das estabelecidas para $M$-estimadores. Estimação da matriz 
de covariâncias assintótica dos estimadores também é considerada. Para a aplicação da técnica do escore corrigido, precisamos em primeiro lugar da existência do escore corrigido; e, além disso, normalidade do erro e conhecimento da matriz de covariâncias do erro. Contudo, neste capítulo também discutimos como estas restrições podem ser evitadas de alguma forma. Obviamente, o problema da existência do escore corrigido é a limitação mais séria, que só poderia ser contornada considerando algum tipo de "escore corrigido aproximado", implicando portanto na obtenção de estimadores "aproximadamente consistentes". Por outro lado, quando a matriz de covariâncias do erro não pode ser assumida conhecida, a disponibilidade de medidas repetidas nas verdadeiras covariáveis proporciona um estimador da mesma. Os resultados assintóticos são modificados nesse caso. A existência do escore corrigido depende fortemente da normalidade do erro. No entanto, mostramos como a consideração de uma distribuição normal contaminada para o erro de medição permite a aplicação da técnica levando em conta a presença de possíveis "outliers" presentes nas covariáveis. Finalmente, os exemplos mostram que a técnica pode ser aplicada a vários modelos de interesse.

O Capítulo 3 é dedicado ao estudo de testes de hipóteses. Baseados nos resultados assintóticos do Capítulo 2, são propostos cinco testes assintóticos, tipo razão de verossimilhança, escore e de Wald. Comparações entre os testes baseados na metodologia do escore corrigido e testes "naive" produzem resultados similares aos encontrados nos trabalhos de Tosteson e Tsiatis (1988) e Stefanski e Carroll (1990), onde testes "naive" são comparados com outros testes alternativos. Dado que o escore corrigido nem sempre existe, precisamos determinar em primeiro lugar uma classe de modelos que admitam escore corrigido, sobre a qual considerar as comparações. Assim, mostramos que para a família Tweedie de modelos (Jørgensen, 1987) com média log-linear, estruturais, testes "naive" são apropriados quando testamos a hipótese de não associação entre uma variável resposta e uma covariável. Um outro resultado interessante para esta família de modelos é 
obtido quando assumimos que as verdadeiras covariáveis são normalmente distribuídas. $\mathrm{O}$ fator de atenuação no estimador, como conseqüência do erro de medição, pode ser obtido explicitamente e coincide com o fator conhecido para o modelo de regressão linear. Embora o efeito da atenuação tenha sido observado, na literatura não encontramos cálculos explícitos do fator de atenuação fora do modelo de regressão linear. Finalmente, alguns estudos de simulação ilustram o comportamento dos testes para amostras pequenas, sob diferentes valores da variância do erro.

No Capítulo 4, aplicamos a técnica do escore corrigido para fazer inferência no modelo de calibração comparativa, estrutural e funcional. Mostramos como o comportamento sob a seqüência de parâmetros incidentais no modelo funcional é determinado pelas condições de regularidade impostas para a validade dos resultados assintóticos. Notamos que essas suposições sobre o comportamento das verdadeiras covariáveis são mais fracas do que as estabelecidas na literatura para o modelo de regressão linear. Duas condições de identificabilidade são consideradas para este modelo; a variância do erro $\sigma_{u}^{2}$, conhecida, e as razões de variâncias $\lambda_{k}=\sigma_{k}^{2} / \sigma_{u}^{2}, k=1, \ldots, p$, conhecidas. No primeiro caso, o estimador do escore corrigido é facilmente obtido maximizando a log-verossimilhança corrigida. Por outro lado, mostramos que a segunda condição de identificabilidade implica num modelo para o qual o método do escore corrigido pode ainda ser aplicado, sem considerar $\sigma_{u}^{2}$ conhecida, nem estimada previamente como assumido na formulação do método, no Capítulo 2. A variância do erro pode ser estimada juntamente com os outros parâmetros do modelo. O estimador é facilmente obtido por um método iterativo, no entanto, deve-se ter cuidado especial com o problema da existência de raízes múltiplas do escore corrigido. No caso $p=2$, soluções explícitas são encontradas. Os estimadores são comparados com os estimadores de momentos e de máxima verossimilhança. A matriz de covariâncias assintótica é obtida sob as duas condições de identificabilidade. No cálculo desta matriz, a suposição de existência do quarto momento é requerida no lugar de normalidade. Testes de Wald 
e escore baseados no escore corrigido são propostos para testar a igualdade das linhas de calibração. O modelo de regressão linear simples é derivado como caso particular sob ambas as condições de identificabilidade. Nesse caso, os estimadores do escore corrigido coincidem com os estimadores consistentes encontrados na literatura, sejam de máxima verossimilhança ou de momentos.

No Capítulo 5, estudamos o modelo de regressão Weibull estrutural na presença de observações censuradas. O interesse aqui é comparar o estimador do escore corrigido com outros propostos na literatura, através de um estudo de simulação. Além disso, propomos um novo estimador, baseado no cálculo explícito do vício assintótico do estimador "naive", que resulta consistente na ausência de censura. Resultados sobre testes de hipóteses, análogos aos apresentados no Capítulo 3 são obtidos para o modelo Weibull. 


\section{Capítulo 2}

\section{O método do escore corrigido}

\subsection{Introdução}

O método do escore corrigido para estimação em modelos com erros nas variáveis foi proposto por Nakamura (1990). Uma vantagem significativa deste enfoque é sua aplicabilidade tanto a modelos funcionais quanto estruturais. No entanto, é particularmente apropriado para modelos funcionais devido ao fato de que a função escore corrigida não envolve as verdadeiras covariáveis desconhecidas ou parâmetros incidentais do modelo. Conseqüentemente, esses parâmetros de perturbação não precisam ser estimados para a obtenção dos estimadores dos parâmetros estruturais do modelo.

O problema da estimação dos parâmetros estruturais na presença de um grande número de parâmetros "nuisance" foi primeiro considerado por Neyman e Scott (1948). Eles mostraram que os estimadores de máxima verossimilhança (EMV) dos parâmetros estruturais do modelo podem ser inconsistentes. Por outro lado, a matriz de covariâncias assintótica não é necessariamente dada pela inversa da matriz de informação de Fisher, como mostrado por Patefield $(1977,1978)$.

Stefanski e Carroll (1985) mostram que, em regressão logística, os EMV dos parâmetros estruturais em um modelo funcional são inconsistentes e difíceis de serem obtidos. A inconsistência de EMV em modelos funcionais também foi observada por Theobald e 
Mallinson (1978).

O fracasso do método de máxima verossimilhança em modelos funcionais para se obter estimadores consistentes tem motivado a busca de enfoques alternativos para estimação.

Vários trabalhos têm se concentrado na obtenção de equações de estimação não viciadas que não dependem dos parâmetros incidentais do modelo. Em alguns casos é possível explorar a estrutura particular do modelo. Assim, condicionando em certas estatísticas suficientes para os parâmetros "nuisance", equações de estimação não viciadas que podem levar a estimadores consistentes, são obtidas a partir da distribuição condicional. Nesta direção podemos citar os trabalhos de Andersen (1970) e Stefanski e Carroll (1987).

A técnica do escore corrigido precisa, em geral, de uma estrutura aditiva de erros de medição, do conhecimento da matriz de covariâncias associada ao erro, ou de um estimador desta matriz, e, obviamente, da existência da função escore corrigida. Por sua vez, a suposição de normalidade no erro de medição, permite obter condições suficientes para a existência e unicidade da função escore corrigida. A propriedade fudamental que define uma função escore corrigida é o fato de que sua esperança com respeito à distribuição dos erros de medição, coincide com a função escore usual baseada nas verdadeiras covariáveis desconhecidas. Esta propriedade é a chave para provar a consistência e a normalidade assintótica do estimador obtido como raiz do escore corrigido.

O escore corrigido proposto por Nakamura é definido como o gradiente de uma função de log-verossimilhança corrigida. Esta definição implica na suposição de que a diferenciação da log-verossimilhança corrigida com respeito aos parâmetros estruturais do modelo e, a esperança condicional com respeito à distribuição do erro de medição dadas as variáveis resposta e as verdadeiras covariáveis, sejam operações permutáveis. No entanto, num modelo simples e prático, como o modelo de regressão linear simples, normal, com razão de variâncias conhecida, é fácil ver que o escore corrigido não pode ser obtido como o gradiente de uma log-verossimilhança corrigida, mesmo existindo esta função. Contudo, 
um escore corrigido pode ser encontrado diretamente a partir do escore usual baseado nas verdadeiras covariáveis.

O escore corrigido considerado neste trabalho é construído sem referência a uma função de log-verossimilhança corrigida, mas baseado diretamente no escore usual, função das verdadeiras covariáveis. Definida desta forma, a técnica resulta mais geral do que a proposta por Nakamura. $\checkmark$

Segue da definição que todo escore corrigido é também um escore não viciado, i.e., tem esperança zero quando avaliado no verdadeiro valor do parâmetro. Stefanski e Carroll (1987) consideram escores não viciados, obtidos condicionando em certas estatísticas suficientes dependentes dos parâmetros. É claro que um escore não viciado não é necessariamente um escore corrigido, como também foi notado por Nakamura (1990).

$\mathrm{Na}$ Seção 2.2 apresentamos o método sob a forma de um modelo funcional. As modificações para o caso estrutural são imediatas e serão mencionadas no decorrer da exposição.

Na Seção 2.3 são estudadas as propriedades assintóticas dos estimadores no caso em que a matriz de covariâncias do erro de medição é conhecida. São estabelecidas condições de regularidade suficientes para as provas da consistência e normalidade assintótica. A estimação consistente da matriz de covariâncias assintótica dos estimadores, também é discutida. As modificações necessárias aos resultados assintóticos, quando um estimador da matriz de covariâncias do erro é disponível, são dadas na Seção 2.4. É considerada a situação particular em que este estimador é obtido a partir de medições replicadas das verdadeiras covariáveis.

A técnica fica bem definida uma vez que possa ser provada a existência e unicidade da função escore corrigida. Uma tal função nem sempre existe. Condições para existência e unicidade no caso em que a distribuição do erro de medição é normal, são apresentadas na Seção 2.5, baseado no trabalho de Stefanski (1989).

O método pode ser aplicado a uma ampla classe de modelos lineares generalizados. 
Alguns exemplos são apresentados na Seção 2.6.

Como conseqüência dos resultados da Seção 2.5, o método do escore corrigido dependeria fortemente da suposição de normalidade no erro de medição. Entretanto, é sabido que a inferência estatística baseada na distribuição normal é vulnerável a "outliers". A distribuição normal contaminada produz um modelo apropriado quando os dados nas covariáveis observadas são contaminados com uma pequena fração de "outliers". Na Seção 2.7 é dada uma extensão do método do escore corrigido, supondo uma distribuição normal contaminada para o erro de medição.

\subsection{Descrição do método}

Consideremos $y$ um vetor $r \times 1$ de variáveis resposta, $z$ um vetor $q \times 1$ de covariáveis e $\theta$ um vetor $p \times 1$ de parâmetros desconhecido que desejamos estimar, pertencente ao espaço paramétrico $\Theta$.

A inferência será baseada numa amostra de $n$ observações independentes. Denotaremos por $Z=\left(z_{1}, \ldots, z_{n}\right)^{\prime}$, a matriz de covariáveis e por $Y=\left(y_{1}, \ldots, y_{n}\right)^{\prime}$, a matriz de variáveis resposta.

Seja

$$
\ell(\theta ; Z, Y)=\sum_{i=1}^{n} \ell_{i}\left(\theta ; z_{i}, y_{i}\right)
$$

a função de log-verossimilhança de $\theta$, dados $Z$ e $Y$,

$$
U(\theta ; Z, Y)=\frac{\partial}{\partial \theta} \ell(\theta ; Z, Y)
$$

e

$$
I(\theta ; Z, Y)=-\frac{\partial}{\partial \theta} U(\theta ; Z, Y)
$$

a função escore e a matriz de informação observada, respectivamente, desde que as derivadas existam, $\forall \theta \in \mathcal{F}, \forall Y, Z$, onde $\mathcal{F}$ é um subconjunto aberto e conexo de $\Theta$. 
Sob condições de regularidade apropriadas (veja por exemplo Serfling, 1980), o estimador de máxima verossimilhança $(\mathrm{EMV}), \hat{\theta}_{Z}$, pode ser obtido via maximização da log-verossimilhança, o que implica na resolução da equação de estimação

$$
U(\theta ; Z, Y)=0
$$

É sabido (Serfling, 1980) que, sob condições de regularidade apropriadas, $\widehat{\theta}_{Z}$ é consistente e assintoticamente normal, com vetor de médias $\theta_{0}$ e matriz de covariâncias assintótica dada por $\left\{E\left[I\left(\theta_{0} ; Z, Y\right)\right]\right\}^{-1}$, onde $\theta_{0}$ é o verdadeiro valor do parâmetro. Este fato é baseado fortemente na seguinte propriedade do escore:

$$
E\left[U\left(\theta_{0} ; Z, Y\right)\right]=0
$$

sendo $\theta_{0}$ o único valor de $\theta$ que anula a esperança acima.

Consideremos agora que $z$ não pode ser observada diretamente por estar sujeita a erro de medição, e denotemos por $x$ ao valor observado de $z$.

Num modelo com erros aditivos, escrevemos

$$
x_{i}=z_{i}+u_{i}, \quad i=1, \ldots, n,
$$

onde $u_{i}$ é o erro de medição associado à $i$-ésima observação. Os $u_{i}$ 's são vetores aleatórios independentes, independentes dos $z_{i}$ 's, com

$$
E\left[u_{i} \mid z_{i}, y_{i}\right]=0 \quad \text { e } \quad E\left[u_{i} u_{i}^{\prime} \mid z_{i}, y_{i}\right]=\Sigma_{u}, \quad i=1, \ldots, n
$$

onde $\Sigma_{u}$ é uma matriz $q \times q$ positiva semidefinida, que assumiremos conhecida. A situação onde $\Sigma_{u}$ é desconhecida será considerada na Seção 2.4 .

Quando as covariáveis $z_{i}$ 's são constantes desconhecidas, um modelo funcional é obtido; se as $z_{i}$ 's são vetores aleatórios independentes e identicamente distribuídos, temos um modelo estrutural. 
A função escore "naive" é obtida simplesmente substituindo $Z$ por $X$ na função escore usual. O EMV "naive" de $\theta_{0}$, que denotaremos por $\hat{\theta}_{X}$, será solução da equação $U(\theta ; X, Y)=0$.

Em geral, $E\left[U\left(\theta_{0} ; X, Y\right)\right] \neq 0$ e, conseqüentemente, $\widehat{\theta}_{X}$ não será necessariamente consistente.

Stefanski (1985) estuda um método para avaliar o vício de $\widehat{\theta}_{X}$ e constrói um estimador com menor vício.

Um estimador consistente de $\theta$ poderia ser obtido como solução de uma equação de estimação não viciada. Nakamura (1990) propõe obter tal função da maneira seguinte: seja $\ell^{*}(\theta ; X, Y)$ uma função das variáveis observadas e do parâmetro desconhecido, tal que

$$
E\left[\ell^{*}(\theta ; X, Y) \mid Z, Y\right]=\ell(\theta ; Z, Y)
$$

$\ell^{*}(\theta ; X, Y)$ é chamada função de log-verossimilhança corrigida.

Se $\ell^{*}(\theta ; X, Y)$ for duas vezes diferenciável em $\mathcal{F}$, definem-se

$$
U^{*}(\theta ; X, Y)=\frac{\partial}{\partial \theta} \ell^{*}(\theta ; X, Y)
$$

e

$$
I^{*}(\theta ; X, Y)=-\frac{\partial}{\partial \theta} U^{*}(\theta ; X, Y)
$$

a função escore corrigida e a matriz de informação observada corrigida, respectivamente.

Se $E[\cdot \mid Z, Y]$ e $\frac{\partial}{\partial \theta}$ forem operações permutáveis,

$$
E\left[U^{*}(\theta ; X, Y) \mid Z, Y\right]=U(\theta ; Z, Y), \forall \theta \in \mathcal{F}
$$

O valor $\widehat{\theta}$ tal que $U^{*}(\widehat{\theta} ; X, Y)=0$ e $I^{*}(\widehat{\theta} ; X, Y)$ é positiva definida, é dito estimador do escore corrigido de $\theta$.

Das propriedades (2.2) e (2.4), temos que

$$
E\left[U^{*}\left(\theta_{0} ; X, Y\right)\right]=E\left[E\left[U^{*}\left(\theta_{0} ; X, Y\right) \mid Z, Y\right]\right]=E\left[U\left(\theta_{0} ; Z, Y\right)\right]=0
$$


i.e., $U^{*}(\theta ; X, Y)$ é um escore não viciado. Esta última propriedade e condições de regularidade apropriadas garantem a consistência e a normalidade assintótica de $\hat{\theta}$. No entanto, note que se $E[\cdot \mid Z, Y]$ e $\frac{\partial}{\partial \theta}$ não forem operações permutáveis, a propriedade (2.4) não seria obtida e, conseqüentemente, $U^{*}(\theta ; X, Y)$ não seria necessariamente não viciado. Esta situação é ilustrada no exemplo seguinte para um modelo simples e prático.

Exemplo 2.1 Modelo de regressão linear simples com razão de variâncias conhecida

Considere o modelo representado pelas equações

$$
\begin{aligned}
& y_{i}=\alpha+\beta z_{i}+\varepsilon_{i} \\
& x_{i}=z_{i}+u_{i}
\end{aligned}
$$

$\operatorname{com} \varepsilon_{i} \stackrel{\text { iid }}{\sim} N\left(0, \sigma_{\varepsilon}^{2}\right)$ e $u_{i} \stackrel{\text { iid }}{\sim}\left(0, \sigma_{u}^{2}\right), i=1, \ldots, n$.

Uma das suposições que torna o modelo identificável é o conhecimento da ṛ̇zão $\lambda=$ $\sigma_{\varepsilon}^{2} / \sigma_{u}^{2}$. Sem perda de generalidade, $\lambda$ pode ser tomada igual a 1 , de modo que $\sigma_{\varepsilon}^{2}=$ $\sigma_{u}^{2}=\phi$. O parâmetro estrutural do modelo é $\theta=(\alpha, \beta, \phi)^{\prime}$ e os parâmetros incidentais $Z=\left(z_{1}, \ldots, z_{n}\right)^{\prime}$.

A função de log-verossimilhança "não observada" é dada por

$$
\ell(\theta ; Z, Y)=\sum_{i=1}^{n} \ell_{i}\left(\theta ; z_{i}, y_{i}\right)
$$

onde

$$
\ell_{i}\left(\theta ; z_{i}, y_{i}\right)=\text { cte. }-\frac{1}{2} \log \phi-\frac{1}{2 \phi}\left(y_{i}-\alpha-\beta z_{i}\right)^{2}, i=1, \ldots, n
$$

de modo que a função escore "não observada" é dada por

$$
U(\theta ; Z, Y)=\sum_{i=1}^{n} U_{i}\left(\theta ; z_{i}, y_{i}\right)
$$

onde $U_{i}=\left(U_{i \alpha}, U_{i \beta}, U_{i \phi}\right)^{\prime}$, com

$$
U_{i \alpha}=\frac{\partial \ell_{i}}{\partial \alpha}=\frac{1}{\phi}\left(y_{i}-\alpha-\beta z_{i}\right)
$$




$$
\begin{aligned}
U_{i \beta} & =\frac{\partial \ell_{i}}{\partial \beta}=\frac{1}{\phi}\left(y_{i}-\alpha-\beta z_{i}\right) z_{i}, \\
U_{i \phi} & =\frac{\partial \ell_{i}}{\partial \phi}=-\frac{1}{2 \phi}+\frac{1}{2 \phi^{2}}\left(y_{i}-\alpha-\beta z_{i}\right)^{2} .
\end{aligned}
$$

É fácil ver que a função de log-verossimilhança corrigida é

$$
\ell^{*}(\theta ; X, Y)=\sum_{i=1}^{n} \ell_{i}^{*}\left(\theta ; x_{i}, y_{i}\right)
$$

onde

$$
\ell_{i}^{*}\left(\theta ; x_{i}, y_{i}\right)=\text { cte. }-\frac{1}{2} \log \phi-\frac{1}{2 \phi}\left(y_{i}-\alpha-\beta x_{i}\right)^{2}+\frac{\beta^{2}}{2}
$$

$\ell^{*}$ é a única função satisfazendo $E\left[\ell^{*}(\theta ; X, Y) \mid Z, Y\right]=\ell(\theta ; Z, Y)$, pois $x_{i}$ e $x_{i}^{2}-\phi$ são os únicos estimadores não viciados de $z_{i}$ e $z_{i}^{2}$, respectivamente.

A função escore corrigida é dada por

$$
U^{*}(\theta ; X, Y)=\sum_{i=1}^{n} U_{i}^{*}\left(\theta ; x_{i}, y_{i}\right)
$$

onde

$$
\begin{aligned}
U_{i \alpha}^{*} & =\frac{\partial \ell_{i}^{*}}{\partial \alpha}=\frac{1}{\phi}\left(y_{i}-\alpha-\beta x_{i}\right), \\
U_{i \beta}^{*} & =\frac{\partial \ell_{i}^{*}}{\partial \beta}=\frac{1}{\phi}\left(y_{i}-\alpha-\beta x_{i}\right) x_{i}+\beta \\
U_{i \phi}^{*} & =\frac{\partial \ell_{i}^{*}}{\partial \phi}=-\frac{1}{2 \phi}+\frac{1}{2 \phi^{2}}\left(y_{i}-\alpha-\beta x_{i}\right)^{2} .
\end{aligned}
$$

Contudo, note que

$$
E\left[U_{i \phi}^{*}\left(\theta ; x_{i}, y_{i}\right) \mid z_{i}, y_{i}\right]=U_{i \phi}\left(\theta ; z_{i}, y_{i}\right)+\frac{\beta^{2}}{2 \phi}
$$

de modo que $E\left[U_{i \phi}^{*}\left(\theta ; x_{i}, y_{i}\right) \mid z_{i}, y_{i}\right] \neq U_{i \phi}\left(\theta ; z_{i}, y_{i}\right), \forall \beta \neq 0$, conseqüentemente a propriedade (2.4) não é verificada.

Dado que a propriedade (2.4) e condições de regularidade apropriadas são suficientes para se obter um escore não viciado, neste trabalho definiremos a função escore corrigida da seguinte maneira: 
Definição 2.1 Uma função $U^{*}(\theta ; X, Y)$ é dita função escore corrigida se

$$
E\left[U^{*}(\theta ; X, Y) \mid Z, Y\right]=U(\theta ; Z, Y), \quad \forall \theta \in \mathcal{F}
$$

Sob certas condições de regularidade, nas próximas seções será provada a existência de uma seqüência consistente e assintoticamente normal $\hat{\theta}_{n}$, de soluções da equação

$$
U^{*}(\theta ; X, Y)=0
$$

\section{Observações}

(1) Uma função escore corrigida, no sentido da Definição 2.1, não é necessariamente o gradiente de uma log-verossimilhança corrigida $\ell^{*}$. Portanto, não é verdade que a

$=\quad$ equação (2.5) defina $\widehat{\theta}$ unicamente como aquele valor de $\theta$ que maximiza $\ell^{*}$. No caso de raízes múltiplas, a escolha da raiz apropriada deve ser feita de maneira cuidadosa, pois podem existir seqüências de soluções que não são consistentes. Algumas possíveis soluções para este problema são discutidas em Stefanski e Carroll (1987) e Stefanski (1989), e serão consideradas no Capítulo 4.

(2) Se a matriz $\Sigma_{u}$ for desconhecida, $U^{*}$ dependerá também deste parâmetro. Indicaremos isto escrevendo $U^{*}\left(\theta, \Sigma_{u} ; X, Y\right)$ quando for necessário enfatizar esta dependência.

(3) Uma das características atrativas do método do escore corrigido é sua aplicabilidade tanto a modelos estruturais quanto funcionais. Note-se que não são colocadas suposições sobre as verdadeiras covariáveis $Z$. Contudo, o método resulta particularmente apropriado para modelos funcionais devido ao fato de que a equação (2.5) não envolve os verdadeiros valores $\left(z_{i}\right)$ ou parâmetros incidentais do modelo. Desta maneira, os $\left(z_{i}\right)$ não precisarão ser estimados para a obtenção dos estimadores dos parâmetros estruturais do modelo. 
(4) Uma função escore corrigida nem sempre existe. O problema da existência e unicidade de uma tal função será discutido na Seção 2.5. Assumiremos no que segue que tal função existe.

(5) Segue da Definição 2.1 que toda função escore corrigida é não viciada, ou seja, $E\left[U^{*}\left(\theta_{0} ; X, Y\right)\right]=0$. A recíproca não é verdadeira, Stefanski e Carroll (1987) consideram escores não viciados obtidos condicionando em certas estatísticas suficientes dependentes dos parâmetros.

(6) A técnica baseada no escore corrigido também pode ser aplicada quando em ausência de erro de medição, o parâmetro de interesse é estimado por um $M$-estimador que resulta consistente. Consideremos então uma outra função $\psi(\theta ; Z, Y)$ no lugar do escore, com a propriedade que $E\left[\psi\left(\theta_{0} ; Z, Y\right)\right]=0$. Um estimador consistente de $\theta$ pode ser obtido na presença de erro de medição, como solução da equação $\psi^{*}(\theta ; X, Y)=0$, desde que exista $\psi^{*}(\theta ; X, Y)$ tal que $E\left[\psi^{*}(\theta ; X, Y) \mid Z, Y\right]=\psi(\theta ; Z, Y), \forall \theta$.

Para completar a notação, seja

$$
U^{*}(\theta ; X, Y)=\sum_{i=1}^{n} U_{i}^{*}\left(\theta ; x_{i}, y_{i}\right)
$$

onde $U_{i}^{*}\left(\theta ; x_{i}, y_{i}\right)=\left(U_{i 1}^{*}\left(\theta ; x_{i}, y_{i}\right), \ldots, U_{i p}^{*}\left(\theta ; x_{i}, y_{i}\right)\right)^{\prime}, i=1, \ldots, n$

$$
I^{*}(\theta ; X, Y)=\sum_{i=1}^{n} I_{i}^{*}\left(\theta ; x_{i}, y_{i}\right)
$$

sendo $I_{i}^{*}\left(\theta ; x_{i}, y_{i}\right)$ a matriz $p \times p$ cujo elemento $(k, l)$ é dado por

$$
I_{i k l}^{*}\left(\theta ; x_{i}, y_{i}\right)=-\frac{\partial}{\partial \theta_{l}} U_{i k}^{*}\left(\theta ; x_{i}, y_{i}\right), k, l=1, \ldots, p, i=1, \ldots, n
$$

Note que as matrizes $I_{i}^{*}\left(\theta ; x_{i}, y_{i}\right)$ não precisam ser simétricas. Por simplicidade de notação, escreveremos no que segue, simplesmente $U^{*}(\theta), U_{i}^{*}(\theta), I^{*}(\theta), I_{i}^{*}(\theta)$ e $I_{i k l}^{*}(\theta), i=1, \ldots, n$, 
$k, l=1, \ldots, p$. Sejam

$$
\begin{aligned}
& \bar{U}_{n}^{*}(\theta)=\frac{1}{n} U^{*}(\theta)=\frac{1}{n} \sum_{i=1}^{n} U_{i}^{*}(\theta), \\
& \bar{\Gamma}_{n}^{*}(\theta)=\frac{1}{n} I^{*}(\theta)=\frac{1}{n} \sum_{i=1}^{n} I_{i}^{*}(\theta), \\
& \bar{\Lambda}_{n}(\theta)=\frac{1}{n} E\left[I^{*}(\theta)\right]=\frac{1}{n} \sum_{i=1}^{n} E\left[I_{i}^{*}(\theta)\right] \\
& \bar{\Gamma}_{n}(\theta)=\frac{1}{n} \sum_{i=1}^{n} E\left[U_{i}^{*}(\theta) U_{i}^{*}(\theta)^{\prime}\right] .
\end{aligned}
$$

No caso do modelo funcional, as matrizes $\bar{\Lambda}_{n}$ e $\bar{\Gamma}_{n}$ dependerão também da seqüência de parâmetros incidentais $Z=\left(z_{1}, \ldots, z_{n}\right)^{\prime}$. Para o modelo estrutural $\bar{\Lambda}_{n}=\Lambda$ e $\bar{\Gamma}_{n}=$ $\Gamma$, independentes de $n$, mas dependerão de $\theta$ e outros parâmetros que caracterizam a distribuição das covariáveis $z_{i}$.

Por simplicidade, escreveremos simplesmente $\bar{\Lambda}_{n}(\theta)$ e $\bar{\Gamma}_{n}(\theta)$, lembrando sempre a dependência destas matrizes em outros parâmetros envolvidos no modelo, seja este funcional ou estrutural.

\subsection{Propriedades assintóticas dos estimadores}

Mostraremos a seguir que, sob condições de regularidade convenientes, a equação $U^{*}(\theta)=$ 0 tem uma raiz consistente e assintoticamente normal.

\subsubsection{Condições de regularidade}

Condições de regularidade suficientes para a validade dos resultados assintóticos não são apresentadas no trabalho de Nakamura (1990), assumindo-se que a teoria de $M$ estimadores (Huber, 1981) pode ser aplicada.

O modelo funcional, particularmente, requer um tratamento rigoroso das condições de regularidade e provas das propriedades assintóticas dos estimadores. 
Vários autores têm proposto diferentes condições de regularidade sob as quais o enfoque usual de máxima verossimilhança no caso i.i.d. produz estimadores consistentes e assintoticamente normais (veja por exemplo Norden, 1973).

Num modelo funcional, a suposição de observações identicamente distribuídas não é verificada. Para cada $i$, a distribuição de $\left(y_{i}, x_{i}\right)$ dependerá do parâmetro estrutural $\theta$ e do parâmetro incidental $z_{i}$. Na literatura, tem sido estudada a situação onde as observações resultam independentes mas não identicamente distribuídas. No entanto, na maioria dos casos discutidos, não é considerada a existência de parâmetros incidentais (veja Hoadley, 1971, Philippou e Roussas, 1975, e Weiss, 1971, 1973, 1975). Mak (1982) propõe uma técnica de estimação via o enfoque usual de máxima verossimilhança, na presença de parâmetros incidentais, e estabelece condições de regularidade que levam em conta este fato.

Num modelo funcional, é claro que qualquer convergência aos verdadeiros valores dependerá do comportamento assintótico assumido para a seqüência de parâmetros incidentais $\left(z_{i}\right)$. Portanto, as condições de regularidade impostas serão bastante diferentes das usuais no caso i.i.d.

Assumimos que as derivadas

$$
I_{i k l}^{*}\left(\theta ; x_{i}, y_{i}\right)=-\frac{\partial}{\partial \theta_{l}} U_{i k}^{*}\left(\theta ; x_{i}, y_{i}\right), k, l=1, \ldots, p
$$

existem quase certamente, $\forall i$.

C1. Para cada $\theta \in \mathcal{F}$, existe $\delta>0$ e uma função $d_{i k l}\left(=d_{i k l}\left(x_{i}, y_{i}\right)\right)$ tal que

$$
\begin{gathered}
\left|I_{i k l}^{*}\left(\theta^{*}\right)\right|<d_{i k l}, \text { q.c. } \\
i=1, \ldots, n ; k, l=1, \ldots, p, \forall \theta^{*} \in B_{\theta, \delta}=\left\{\theta^{*}:\left\|\theta^{*}-\theta\right\|<\delta\right\} \subset \mathcal{F}, \mathrm{e} \\
0<\limsup \frac{1}{n} \sum_{i=1}^{n} E\left[d_{i k l}^{2}\right]<\infty .
\end{gathered}
$$


C2. $0<\liminf \operatorname{det}\left(\bar{\Lambda}_{n}\left(\theta_{0}\right)\right), \quad 0<\liminf \left\|\bar{\Lambda}_{n}^{-1}\left(\theta_{0}\right)\right\| \leq \limsup \left\|\bar{\Lambda}_{n}^{-1}\left(\theta_{0}\right)\right\|<\infty$, onde para uma matriz $A=\left[a_{i j}\right],\|A\|=\left(\sum_{i} \sum_{j} a_{i j}^{2}\right)^{1 / 2}$ e $\operatorname{det} A \operatorname{denota}$ o determinante de $A$.

C3. Dado $\varepsilon>0$, existe $\delta>0$ tal que

$$
\limsup \frac{1}{n}\left|\sum_{i=1}^{n} E\left[\sup _{\theta \in B_{\theta_{0} ; \delta}} I_{i k l}^{*}(\theta)-I_{i k l}^{*}\left(\theta_{0}\right)\right]\right|<\varepsilon,
$$

$k, l=1, \ldots, p$. Uma condição análoga é assumida com lim inf no lugar de limsup.

C4. Existe $\gamma>0$ tal que, quando $n \rightarrow \infty$,

$$
\frac{1}{n^{1+\frac{\gamma}{2}}} \sum_{i=1}^{n} E\left[\left|U_{i k}^{*}\left(\theta_{0}\right)\right|\right]^{2+\gamma} \rightarrow 0, \quad k=1, \ldots, p .
$$

C5. $0<\liminf \frac{1}{n} \sum_{i=1}^{n} E\left[U_{i k}^{*}\left(\theta_{0}\right) U_{i l}^{*}\left(\theta_{0}\right)\right] \leq \limsup \frac{1}{n} \sum_{i=1}^{n} E\left[U_{i k}^{*}\left(\theta_{0}\right) U_{i l}^{*}\left(\theta_{0}\right)\right]<\infty, k, l=$ $1, \ldots, p$.

\section{Observações}

(1) Se as segundas derivadas parciais de $U_{i}^{*}(\theta)$ existem q.c., então, a condição C3 pode ser substituída por $\mathrm{C}^{*}$, mais fácil de verificar em algumas situações,

C3*. Existe uma função $d_{i k l m}\left(=d_{i k l m}\left(x_{i}, y_{i}\right)\right)$ tal que

$$
\begin{gathered}
\left|\frac{\partial^{2} U_{i k}^{*}(\theta)}{\partial \theta_{l} \partial \theta_{m}}\right|<d_{i k l m} \quad \text { q.c. }, \\
k, l, m=1, \ldots, p, i=1, \ldots n, \forall \theta \in B_{\theta_{0}, \delta}, \text { e } \\
\limsup \frac{1}{n} \sum_{i=1}^{n} E\left[\left|d_{i k l m}\right|\right]<\infty .
\end{gathered}
$$

(2) Num modelo funcional, as propriedades assintóticas do estimador do escore corrigido claramente dependerão do comportamento assintótico dos $z_{i 0}$, verdadeiros $z_{i}$. 
Este comportamento é determinado pelas condições C1 - C5, desde que as matrizes envolvidas dependem de $z_{i 0}$. Note que não é assumido que as matrizes $\bar{\Lambda}_{n}$ e $\bar{\Gamma}_{n}$ convergem a limite nenhum. As condições C2 e C5, respectivamente, determinam o comportamento assintótico destas matrizes.

(3) A condição C4 é estabelecida para a aplicação do Teorema Central do Limite de Liapunov.

(4) No caso de um modelo estrutural, onde $\left(y_{i}, x_{i}\right), i=1, \ldots, n$, são observações i.i.d., as condições de regularidade ficam simplificadas, pois $\bar{\Lambda}_{n}=\Lambda$ e $\bar{\Gamma}_{n}=\Gamma, \forall n$. C4 não será necessária, dado que o Teorema Central do Limite para v.a.i.i.d. poderá ser aplicado.

No que segue, são estudadas as propriedades assintóticas dos estimadores, assumindo um modelo funcional. As modificações necessárias no caso de um modelo estrutural são imediatas.

\subsubsection{Consistência}

Sob as condições de regularidade C1 - C5, provaremos a seguir a existência e unicidade de uma solução consistente da equação $U^{*}(\theta)=0$. A prova é baseada numa modificação da estabelecida por Foutz (1977), para existência e unicidade do EMV no caso i.i.d, que propõe uma aplicação do Teorema da Função Inversa (Rudin, 1964).

O seguinte lema sobre matrizes aleatórias (Mak, 1982) será necessário para a prova da consistência.

Lema 2.1 Seja $\left\{A_{n}\right\}_{n=1}^{\infty}$ uma seqüência de matrizes não singulares $p \times p$ e suponha que existe uma constante $K$ tal que $\left\|A_{n}^{-1}\right\|<K, \forall n$ suficientemente grande. Se $\left\{E_{n}\right\}_{n=1}^{\infty}$ é uma seqüência de matrizes aleatórias tais que $E_{n}-A_{n} \stackrel{\mathrm{p}}{\longrightarrow} 0$, então $E_{n}^{-1}$ existe com probabilidade tendendo a um, quando $n \rightarrow \infty, e\left(E_{n}-A_{n}\right) E_{n}^{-1} \stackrel{\mathrm{p}}{\longrightarrow} 0$. 
Prova: Para uma matriz $A$, considere a norma

$$
\|A\|_{M}=\sup \{\|A X\|:\|X\| \leq 1\}
$$

de modo que $\|A\|_{M} \leq\|A\|$ (Rudin, 1964).

Como $E_{n}-A_{n} \stackrel{\mathrm{p}}{\longrightarrow} 0,\left\|E_{n}-A_{n}\right\| \stackrel{\mathrm{p}}{\longrightarrow} 0$ e $\left\|E_{n}-A_{n}\right\|_{M} \stackrel{\mathrm{p}}{\longrightarrow} 0$, portanto

$$
\left\|E_{n}-A_{n}\right\|<\frac{1}{K}<\frac{1}{\left\|A_{n}^{-1}\right\|}<\frac{1}{\left\|A_{n}^{-1}\right\|_{M}}
$$

com probabilidade tendendo a um, quando $n \rightarrow \infty$. Então, pelo Teorema 9.8(a) (Rudin, 1964), $E_{n}^{-1}$ existe com probabilidade tendendo a um, quando $n \rightarrow \infty$.

É fácil ver que

$$
\left\|E_{n}^{-1}\right\| \leq\left(\left\|A_{n}^{-1}\right\|-\left\|E_{n}-A_{n}\right\|\right)^{-1}<2 K
$$

com probabilidade tendendo a um, quando $n \rightarrow \infty$. Portanto,

$$
\left\|\left(E_{n}-A_{n}\right) E_{n}^{-1}\right\| \leq\left\|E_{n}-A_{n}\right\|\left\|E_{n}^{-1}\right\| \stackrel{\mathrm{p}}{\longrightarrow} 0,
$$

quando $n \rightarrow \infty$. Segue então que

$$
\left(E_{n}-A_{n}\right) E_{n}^{-1} \stackrel{\mathrm{p}}{\longrightarrow} 0 .
$$

Teorema 2.1 Se as condições C1 - C3 e C5 são satisfeitas, então, com probabilidade tendendo a um quando $n \rightarrow \infty, \bar{U}_{n}^{*}(\theta)=0$ tem uma raiz consistente $\hat{\theta}_{n}$. $\hat{\theta}_{n}$ é única no sentido de que, se $\tilde{\theta}_{n}$ é outra raiz consistente, então $\widehat{\theta}_{n}=\tilde{\theta}_{n}$ com probabilidade tendendo a um quando $n \rightarrow \infty$.

Prova: C2 implica que existe $K:\left\|\bar{\Lambda}_{n}^{-1}\left(\theta_{0}\right)\right\|<K, \forall n$ suficientemente grande.

De C1 e a desigualdade de Chebychev temos que existe $\delta>0$ tal que $\forall \theta \in B_{\theta_{0}, \delta}$

$$
\bar{I}_{n}^{*}(\theta)-\bar{\Lambda}_{n}(\theta) \stackrel{\mathrm{p}}{\longrightarrow} 0,
$$


quando $n \rightarrow \infty$, onde $B_{\theta_{0}, \delta}=\left\{\theta:\left\|\theta-\theta_{0}\right\|<\delta\right\}$. Portanto, do Lema 2.1, segue que $\left[\bar{I}_{n}^{*}\left(\theta_{0}\right)\right]^{-1}$ existe com probabilidade tendendo a um, quando $n \rightarrow \infty$, e

$$
\left\|\left[\bar{I}_{n}^{*}\left(\theta_{0}\right)\right]^{-1}\right\|<2 K .
$$

Seja $\lambda=\frac{1}{4 K}$, então, de (2.11) temos que $\lambda<2 \lambda_{n}$ com probabilidade tendendo a um, quando $n \rightarrow \infty$, onde

$$
\lambda_{n}=\frac{1}{4\left\|\left[\bar{I}_{n}^{*}\left(\theta_{0}\right)\right]^{-1}\right\|} .
$$

Por outro lado, C3 implica que

$$
\left\|\bar{\Lambda}_{n}(\theta)-\bar{\Lambda}_{n}\left(\theta_{0}\right)\right\|<\frac{\lambda}{2}
$$

$\forall \theta \in B_{\theta_{0}, \delta} ; \delta$ suficientemente pequeno.

Então, de (2.10) e (2.12) segue que, com probabilidade tendendo a um quando $n \rightarrow \infty$,

$$
\begin{aligned}
\left\|\bar{I}_{n}^{*}(\theta)-\bar{I}_{n}^{*}\left(\theta_{0}\right)\right\| & \leq\left\|\bar{I}_{n}^{*}(\theta)-\bar{\Lambda}_{n}(\theta)\right\|+\left\|\bar{\Lambda}_{n}\left(\theta_{0}\right)-\bar{I}_{n}^{*}\left(\theta_{0}\right)\right\|+\left\|\bar{\Lambda}_{n}(\theta)-\bar{\Lambda}_{n}\left(\theta_{0}\right)\right\| \\
& <\lambda<2 \lambda_{n}
\end{aligned}
$$

$\forall \theta \in B_{\theta_{0}, \delta} ; \delta$ suficientemente pequeno.

Aplicando o Teorema da Função Inversa a $\bar{U}_{n}^{*}$ (veja Rudin, 1964, para uma prova do teorema), temos que

(a) $\bar{U}_{n}^{*}$ é uma função um a um de $B_{\theta_{0}, \delta}$ em $\bar{U}_{n}^{*}\left(B_{\theta_{0}, \delta}\right)$,

(b) $\bar{U}_{n}^{*}\left(B_{\theta_{0}, \delta}\right) \supset B_{\bar{U}_{n}^{*}\left(\theta_{0}\right), \lambda_{n} \lambda} \supset B_{\bar{U}_{n}^{*}\left(\theta_{0}\right), \frac{\lambda \delta}{2}}$

com probabilidade tendendo a um quando $n \rightarrow \infty$.

Do fato $E\left[\bar{U}_{n}^{*}\left(\theta_{0}\right)\right]=0$, de C5 e a desigualdade de Chebychev, segue que

$$
\bar{U}_{n}^{*}\left(\theta_{0}\right) \stackrel{\mathrm{p}}{\longrightarrow} 0
$$

de modo que

$$
\left\|\bar{U}_{n}^{*}\left(\theta_{0}\right)-0\right\|<\frac{\lambda \delta}{2}
$$


com probabilidade tendendo a um quando $n \rightarrow \infty$. Portanto, $0 \in B_{\bar{U}_{n}^{*}\left(\theta_{0}\right), \frac{\lambda \delta}{2}}$ e por (b) $0 \in \bar{U}_{n}^{*}\left(B_{\theta_{0}, \delta}\right)$.

Considere a função inversa

$$
{\overline{U_{n}}}^{*^{-1}}: \bar{U}_{n}^{*}\left(B_{\theta_{0}, \delta}\right) \rightarrow B_{\theta_{0}, \delta}
$$

esta função está bem definida pelo fato (a). Como $0 \in \bar{U}_{n}^{*}\left(B_{\theta_{0}, \delta}\right)$, concluímos que $\bar{U}_{n}^{*^{-1}}(0) \in B_{\theta_{0}, \delta}$, com probabilidade tendendo a um quando $n \rightarrow \infty$. Dado que $\delta$ pode ser tomado arbitrariamente pequeno, $\bar{U}_{n}^{*-1}(0) \stackrel{\mathrm{p}}{\longrightarrow} \theta_{0}$, e podemos tomar $\widehat{\theta}_{n}=\bar{U}_{n}^{*^{-1}}(0)$ como a seqüência consistente.

Como $\bar{U}_{n}^{*}$ é um a um em $B_{\theta_{0}, \delta}$, qualquer outra seqüência de raízes $\left\{\tilde{\theta}_{n}\right\}: \bar{U}_{n}^{*}\left(\tilde{\theta}_{n}\right)=0$, necessariamente cai fora de $B_{\theta_{0}, \delta}$, com probabilidade tendendo a um quando $n \rightarrow \infty$, implicando que $\tilde{\theta}_{n}$ não é consistente.

\subsubsection{Normalidade assintótica}

Teorema 2.2 Seja $\widehat{\theta}_{n}$ uma raiz consistente da equação $\bar{U}_{n}^{*}(\theta)=0$ e $\theta_{0}$ o verdadeiro valor do parâmetro. Então, sob as condições de regularidade $C 1-C 5, \hat{\theta}_{n}$ é assintoticamente normal com vetor de médias $\theta_{0}$ e matriz de covariâncias assintótica dada por $n^{-1} \Omega_{n}$, onde

$$
\Omega_{n}=\left\{\bar{\Lambda}_{n}\left(\theta_{0}\right)\right\}^{-1} \bar{\Gamma}_{n}\left(\theta_{0}\right)\left\{\bar{\Lambda}_{n}\left(\theta_{0}\right)^{\prime}\right\}^{-1}
$$

Prova: Do Teorema do Valor Médio para uma função de $\mathbb{R}^{n}$ em $\mathbb{R}^{m}$ temos que $\forall h=$ $\left(h_{1}, \ldots, h_{p}\right)^{\prime} \in \mathbb{R}^{p}$

$$
\sqrt{n} h^{\prime} \bar{\Gamma}_{n}^{-1 / 2}\left(\theta_{0}\right) \bar{U}_{n}^{*}\left(\theta_{0}\right)=\sqrt{n} h^{\prime} \bar{\Gamma}_{n}^{-1 / 2}\left(\theta_{0}\right) \bar{I}_{n}^{*}\left(\tilde{\theta}_{n}\right)\left(\hat{\theta}_{n}-\theta_{0}\right),
$$

pois $\bar{U}_{n}^{*}\left(\hat{\theta}_{n}\right)=0$, onde $\left\|\tilde{\theta}_{n}-\theta_{0}\right\| \leq\left\|\hat{\theta}_{n}-\theta_{0}\right\|$.

Note que

$$
\sqrt{n} h^{\prime} \bar{\Gamma}_{n}^{-1 / 2}\left(\theta_{0}\right) \bar{U}_{n}^{*}\left(\theta_{0}\right)=\sum_{i=1}^{n} \frac{1}{\sqrt{n}} h^{\prime} \bar{\Gamma}_{n}^{-1 / 2}\left(\theta_{0}\right) U_{i}^{*}\left(\theta_{0}\right)=\sum_{i=1}^{n} W_{i},
$$


onde $W_{i}=\frac{1}{\sqrt{n}} h^{\prime} \bar{\Gamma}_{n}^{-1 / 2}\left(\theta_{0}\right) U_{i}^{*}\left(\theta_{0}\right), i=1, \ldots, n$, são variáveis aleatórias independentes, com $E\left[W_{i}\right]=0$, desde que $E\left[U_{i}^{*}\left(\theta_{0}\right)\right]=0, i=1, \ldots, n$, e $v_{n}^{2}=\sum_{i=1}^{n} \operatorname{Var}\left[W_{i}\right]=h^{\prime} h$.

Seja $\gamma>0$

$$
\begin{aligned}
\frac{1}{v_{n}^{2+\gamma} \sum_{i=1}^{n} E\left[\left|W_{i}\right|^{2+\gamma}\right]} & =\frac{1}{\left(h^{\prime} h\right)^{1+\frac{\gamma}{2}}} \sum_{i=1}^{n} E\left[\left|\frac{1}{\sqrt{n}} h^{\prime} \bar{\Gamma}_{n}^{-1 / 2}\left(\theta_{0}\right) U_{i}^{*}\left(\theta_{0}\right)\right|^{2+\gamma}\right] \\
& =\frac{1}{\left(h^{\prime} h\right)^{1+\frac{\gamma}{2}} n^{1+\frac{\gamma}{2}}} \sum_{i=1}^{n} E\left[\left|h^{\prime} \bar{\Gamma}_{n}^{-1 / 2}\left(\theta_{0}\right) U_{i}^{*}\left(\theta_{0}\right)\right|^{2+\gamma}\right] \\
& \leq \frac{\left\|h^{\prime} \bar{\Gamma}_{n}^{-1 / 2}\left(\theta_{0}\right)\right\|^{2+\gamma}}{\left(h^{\prime} h\right)^{1+\frac{\gamma}{2}} n^{1+\frac{\gamma}{2}}} \sum_{i=1}^{n} E\left[\left\|U_{i}^{*}\left(\theta_{0}\right)\right\|^{2+\gamma}\right] \\
& \leq \frac{\text { cte. }}{n^{1+\frac{\gamma}{2}}} \sum_{i=1}^{n} \sum_{k=1}^{p} E\left[\left|U_{i k}^{*}\left(\theta_{0}\right)\right|^{2+\gamma}\right] \rightarrow 0,
\end{aligned}
$$

quando $n \rightarrow \infty$, pela condição C4. A última desigualdade segue da condição C5 e do resultado (8a), p.149, de Rao (1973).

Portanto, pelo Teorema Central do Limite de Liapunov,

$$
\frac{\sum_{i=1}^{n} W_{i}}{v_{n}} \stackrel{\mathcal{D}}{\longrightarrow} N(0,1)
$$

equivalentemente,

$$
\sqrt{n} h^{\prime} \bar{\Gamma}_{n}^{-1 / 2}\left(\theta_{0}\right) \bar{U}_{n}^{*}\left(\theta_{0}\right) \stackrel{\mathcal{D}}{\longrightarrow} N\left(0, h^{\prime} h\right),
$$

e, portanto, de (2.13) segue que

$$
\bar{\Gamma}_{n}^{-1 / 2}\left(\theta_{0}\right) \bar{I}_{n}^{*}\left(\tilde{\theta}_{n}\right) \sqrt{n}\left(\widehat{\theta}_{n}-\theta_{0}\right) \stackrel{\mathcal{D}}{\longrightarrow} N_{p}(0, I) .
$$

Dado $\varepsilon>0$ e $\delta$ como na condição $\mathrm{C} 3$, com $\varepsilon / 2$ no lugar de $\varepsilon$, temos, da condição $\mathrm{C} 1$ e da desigualdade de Chebychev, que

$$
P\left\{\left|\frac{1}{n} \sup _{\theta \in B_{\theta_{0}, \delta}} I_{i k l}^{*}(\theta)-\frac{1}{n} E\left[\sup _{\theta \in B_{\theta_{0}, \delta}} I_{i k l}^{*}(\theta)\right]\right|<\frac{\varepsilon}{2}\right\} \rightarrow 1,
$$

quando $n \rightarrow \infty$. Um resultado análogo vale quando sup é substituído por inf.

De (2.15) e do fato de que $\hat{\theta}_{n} \stackrel{\mathrm{p}}{\longrightarrow} \theta_{0}$, quando $n \rightarrow \infty$, segue que

$$
\begin{aligned}
& P\left\{\left|\frac{1}{n} \sum_{i=1}^{n} E\left[I_{i k l}^{*}\left(\theta_{0}\right)\right]-\frac{1}{n} \sum_{i=1}^{n} I_{i k l}^{*}\left(\tilde{\theta}_{n}\right)\right|<\varepsilon\right\} \\
\geq & P\left\{\left|\frac{1}{n} \sum_{i=1}^{n} \inf _{\theta \in B_{\theta_{0}, \delta}} I_{i k l}^{*}(\theta)-\frac{1}{n} \sum_{i=1}^{n} E\left[\inf _{\theta \in B_{\theta_{0}, \delta}} I_{i k l}^{*}(\theta)\right]\right|<\frac{\varepsilon}{2},\right.
\end{aligned}
$$




$$
\left.\left|\frac{1}{n} \sum_{i=1}^{n} \sup _{\theta \in B_{\theta_{0}, \delta}} I_{i k l}^{*}(\theta)-\frac{1}{n} \sum_{i=1}^{n} E\left[\sup _{\theta \in B_{\theta_{0}, \delta}} I_{i k l}^{*}(\theta)\right]\right|<\frac{\varepsilon}{2},\left\|\widehat{\theta}_{n}-\theta_{0}\right\|<\delta\right\} \rightarrow 1,
$$

quando $n \rightarrow \infty$, de modo que

$$
P\left\{\left|\frac{1}{n} \sum_{i=1}^{n} E\left[I_{i k l}^{*}\left(\theta_{0}\right)\right]-\frac{1}{n} \sum_{i=1}^{n} I_{i k l}^{*}\left(\tilde{\theta}_{n}\right)\right|<\varepsilon\right\} \rightarrow 1,
$$

quando $n \rightarrow \infty$. Portanto,

$$
\bar{\Lambda}_{n}\left(\theta_{0}\right)-\bar{I}_{n}^{*}\left(\tilde{\theta}_{n}\right) \stackrel{\mathrm{p}}{\longrightarrow} 0,
$$

quando $n \rightarrow \infty$.

(2.16), C2 e o Lema 2.1 implicam que $\left[\bar{T}_{n}^{*}\left(\tilde{\theta}_{n}\right)\right]^{-1}$ existe e

$$
\left[\bar{\Lambda}_{n}\left(\theta_{0}\right)-\bar{I}_{n}^{*}\left(\tilde{\theta}_{n}\right)\right]\left[\bar{I}_{n}^{*}\left(\tilde{\theta}_{n}\right)\right]^{-1} \stackrel{\mathrm{p}}{\longrightarrow} 0
$$

quando $n \rightarrow \infty$, portanto,

$$
\begin{aligned}
& \bar{\Gamma}_{n}^{-1 / 2}\left(\theta_{0}\right) \bar{\Lambda}_{n}\left(\theta_{0}\right)\left[\bar{I}_{n}^{*}\left(\tilde{\theta}_{n}\right)\right]^{-1} \bar{\Gamma}_{n}^{1 / 2}\left(\theta_{0}\right) \\
= & I+\bar{\Gamma}_{n}^{-1 / 2}\left(\theta_{0}\right)\left[\bar{\Lambda}_{n}\left(\theta_{0}\right)-\bar{I}_{n}^{*}\left(\widetilde{\theta}_{n}\right)\right]\left[\bar{I}_{n}^{*}\left(\tilde{\theta}_{n}\right)\right]^{-1} \bar{\Gamma}_{n}^{1 / 2}\left(\theta_{0}\right) \stackrel{\mathrm{p}}{\longrightarrow} I,
\end{aligned}
$$

quando $n \rightarrow \infty$, pela condição C5 e (2.17).

Finalmente, de (2.14) e (2.18), temos que

$$
\begin{aligned}
& \bar{\Gamma}_{n}^{-1 / 2}\left(\theta_{0}\right) \bar{\Lambda}_{n}\left(\theta_{0}\right) \sqrt{n}\left(\hat{\theta}_{n}-\theta_{0}\right) \\
= & \left\{\bar{\Gamma}_{n}^{-1 / 2}\left(\theta_{0}\right) \bar{\Lambda}_{n}\left(\theta_{0}\right)\left[\bar{I}_{n}^{*}\left(\tilde{\theta}_{n}\right)\right]^{-1} \bar{\Gamma}_{n}\left(\theta_{0}\right)\right\}\left\{\bar{\Gamma}_{n}^{-1 / 2}\left(\theta_{0}\right) \bar{I}_{n}^{*}\left(\tilde{\theta}_{n}\right) \sqrt{n}\left(\hat{\theta}_{n}-\theta_{0}\right)\right\} \stackrel{\mathcal{D}}{\longrightarrow} N_{p}(0, I),
\end{aligned}
$$

o que prova o teorema.

\subsubsection{Estimação da matriz de covariâncias assintótica}

A seguir mostraremos que $\left\{\bar{I}_{n}^{*}(\widehat{\theta})\right\}^{-1} \bar{S}_{n}(\hat{\theta})\left\{\bar{I}_{n}^{*}(\hat{\theta})^{\prime}\right\}^{-1}$ é um estimador consistente de $\left\{\bar{\Lambda}_{n}\left(\theta_{0}\right)\right\}^{-1} \bar{\Gamma}_{n}\left(\theta_{0}\right)\left\{\bar{\Lambda}_{n}\left(\theta_{0}\right)^{\prime}\right\}^{-1}$, onde

$$
\bar{S}_{n}(\theta)=\frac{1}{n} \sum_{i=1}^{n} U_{i}^{*}(\theta) U_{i}^{*}(\theta)^{\prime}
$$


e

$$
\bar{I}_{n}^{*}(\theta)=\frac{1}{n} \sum_{i=1}^{n} I_{i}^{*}(\theta) .
$$

Dado que não estamos assumindo que as matrizes $\bar{\Lambda}_{n}$ e $\bar{\Gamma}_{n}$ convergem a limite nenhum, precisaremos do seguinte lema:

Lema 2.2 Seja $\left\{A_{n}\right\}_{n=1}^{\infty}$ uma seqüência de matrizes não singulares $p \times p$ e suponha que existe uma constante $K$ tal que $\left\|A_{n}^{-1}\right\|<K, \forall n$ suficientemente grande, e $\left\{B_{n}\right\}_{n=1}^{\infty}$ uma seqüência de matrizes $p \times p$ com $0<\liminf \left(B_{n}\right)_{k l} \leq \lim \sup \left(B_{n}\right)_{k l}<\infty, k, l=1, \ldots, p$. Se $\left\{E_{n}\right\}_{n=1}^{\infty} e\left\{D_{n}\right\}_{n=1}^{\infty}$ são seqüências de matrizes aleatórias tais que $E_{n}-A_{n} \stackrel{\mathrm{p}}{\longrightarrow} 0$ e $D_{n}-B_{n} \stackrel{\mathrm{p}}{\longrightarrow} 0$, quando $n \rightarrow \infty$, então

$$
E_{n}^{-1} D_{n}\left(E_{n}^{\prime}\right)^{-1}-A_{n}^{-1} B_{n}\left(A_{n}^{\prime}\right)^{-1} \stackrel{\mathrm{p}}{\longrightarrow} 0
$$

quando $n \rightarrow \infty$.

Prova: Como $E_{n}-A_{n} \stackrel{\mathrm{p}}{\longrightarrow} 0$, segue do Lema 2.1 que $\left(E_{n}-A_{n}\right) E_{n}^{-1} \stackrel{\mathrm{p}}{\longrightarrow} 0$, equivalentemente,

$$
A_{n} E_{n}^{-1} \stackrel{\mathrm{p}}{\longrightarrow} I \quad \text { e } \quad E_{n} A_{n}^{-1} \stackrel{\mathrm{p}}{\longrightarrow} I
$$

de modo que $A_{n} E_{n}^{-1}\left(D_{n}-B_{n}\right)\left(E_{n}^{\prime}\right)^{-1} A_{n}^{\prime} \stackrel{\mathrm{p}}{\longrightarrow} 0$, pois $D_{n}-B_{n} \stackrel{\mathrm{p}}{\longrightarrow} 0$; equivalentemente,

$$
A_{n} E_{n}^{-1} D_{n}\left(E_{n}^{\prime}\right)^{-1} A_{n}^{\prime}-A_{n} E_{n}^{-1} B_{n}\left(E_{n}^{\prime}\right)^{-1} A_{n}^{\prime} \stackrel{\mathrm{p}}{\longrightarrow} 0 .
$$

Por outro lado,

$$
D_{n}-A_{n} A_{n}^{-1} B_{n}\left(A_{n}^{\prime}\right)^{-1} A_{n}^{\prime} \stackrel{\mathrm{p}}{\longrightarrow} 0 .
$$

De (2.20) e (2.21) segue que

$$
A_{n}\left[E_{n}^{-1} D_{n}\left(E_{n}^{\prime}\right)^{-1}-A_{n}^{-1} B_{n}\left(A_{n}^{\prime}\right)^{-1}\right] A_{n}^{\prime}+\left(D_{n}-A_{n} E_{n}^{-1} B_{n}\left(E_{n}^{\prime}\right)^{-1} A_{n}^{\prime}\right) \stackrel{\mathrm{p}}{\longrightarrow} 0 .
$$

(2.19) implica que

$$
E_{n} A_{n}^{-1} D_{n}-D_{n} \stackrel{\mathrm{p}}{\longrightarrow} 0 \quad \text { e } \quad B_{n}-B_{n}\left(E_{n}^{\prime}\right)^{-1} A_{n}^{\prime} \stackrel{\mathrm{p}}{\longrightarrow} 0,
$$


e portanto

$$
\left[E_{n} A_{n}^{-1} D_{n}-B_{n}\left(E_{n}^{\prime}\right)^{-1} A_{n}^{\prime}\right]+\left(B_{n}-D_{n}\right) \stackrel{\mathrm{p}}{\longrightarrow} 0
$$

Assim, $E_{n} A_{n}^{-1} D_{n}-B_{n}\left(E_{n}^{\prime}\right)^{-1} A_{n}^{\prime} \stackrel{\mathrm{p}}{\longrightarrow} 0$, pois $B_{n}-D_{n} \stackrel{\mathrm{p}}{\longrightarrow} 0$; ou equivalentemente por (2.19)

$$
D_{n}-A_{n} E_{n}^{-1} B_{n}\left(E_{n}^{\prime}\right)^{-1} A_{n}^{\prime} \stackrel{\mathrm{p}}{\longrightarrow} 0 .
$$

Segue de (2.22) que

$$
A_{n}\left[E_{n}^{-1} D_{n}\left(E_{n}^{\prime}\right)^{-1}-A_{n}^{-1} B_{n}\left(A_{n}^{\prime}\right)^{-1}\right] A_{n}^{\prime} \stackrel{\mathrm{p}}{\longrightarrow} 0 .
$$

Denotando $C_{n}=E_{n}^{-1} D_{n}\left(E_{n}^{\prime}\right)^{-1}-A_{n}^{-1} B_{n}\left(A_{n}^{\prime}\right)^{-1}$, de $(2.23)$ e o fato que $\left\|A_{n}^{-1}\right\|<K, \forall n$ suficientemente grande, temos que

$$
\left\|C_{n}\right\| \leq\left\|A_{n}^{-1}\right\|\left\|A_{n} C_{n} A_{n}^{\prime}\right\|\left\|\left(A_{n}^{\prime}\right)^{-1}\right\| \stackrel{\mathrm{p}}{\longrightarrow} 0
$$

Portanto, $C_{n} \stackrel{\mathrm{p}}{\longrightarrow} 0$.

Agora, a condição C5, a lei fraca dos grandes números e o fato que $\widehat{\theta} \stackrel{\mathrm{p}}{\longrightarrow} \theta_{0}$, implicam que

$$
\bar{S}_{n}(\widehat{\theta})-\bar{\Gamma}_{n}\left(\theta_{0}\right) \stackrel{\mathrm{p}}{\longrightarrow} 0 .
$$

Por outro lado, das condições C1, C3, a desigualdade de Chebychev e o fato que $\hat{\theta} \stackrel{\mathrm{P}}{\longrightarrow} \theta_{0}$, temos que

$$
\bar{I}_{n}^{*}(\widehat{\theta})-\bar{\Lambda}_{n}\left(\theta_{0}\right) \stackrel{\mathrm{p}}{\longrightarrow} 0
$$

Portanto, de (2.24), (2.25), as condições C2 e C5 e o resultado do Lema 2.2 temos que $\left\{\bar{I}_{n}^{*}(\widehat{\theta})\right\}^{-1} \bar{S}_{n}(\hat{\theta})\left\{\bar{I}_{n}^{*}(\hat{\theta})^{\prime}\right\}^{-1}$ é um estimador consistente de $\left\{\bar{\Lambda}_{n}\left(\theta_{0}\right)\right\}^{-1} \bar{\Gamma}_{n}\left(\theta_{0}\right)\left\{\bar{\Lambda}_{n}\left(\theta_{0}\right)^{\prime}\right\}^{-1}$, chamado de "estimador sanduíche". 


\subsection{Estimação quando a matriz de covariâncias do erro é desconhecida}

Quando $\Sigma_{u}$ é desconhecida são necessários dados adicionais para estimar $\Sigma_{u}$ consistentemente. Por exemplo, medições replicadas dos $z_{i}$ 's podem ser utilizadas para obter um estimador $\widehat{\Sigma}_{u}$. Conseqüentemente, devemos avaliar como a incerteza sobre a variância do erro de medição afetará a precisão do estimador $\hat{\theta}$.

Um problema de interesse ocorre quando para cada $i=1, \ldots, n$ existem $k_{i}$ réplicas independentes de $x_{i}, x_{i j}=z_{i}+u_{i j}, j=1, \ldots, k_{i}$. A situação mais comum é que muitos dos dados não sejam replicados $\left(k_{i}=1\right)$ e outros tenham somente uma réplica $\left(k_{i}=2\right)$ (veja Fuller, 1995). Se $u_{i j}, j=1, \ldots, k_{i}$, são independentes com distribuição $N\left(0, \Sigma_{u}\right)$, então $\bar{x}_{i}=\frac{1}{k_{i}} \sum_{j=1}^{k_{i}} x_{i j}$ tèm distribuição $N\left(z_{i}, \Sigma_{u} / k_{i}\right)$.

Denotemos por $\gamma=\operatorname{Vech}\left(\Sigma_{u}\right)$, onde "vech" é o vetor coluna contendo os elementos distintos de uma matriz simétrica. Suponha que $\widehat{\gamma}=\operatorname{Vech}\left(\widehat{\Sigma}_{u}\right)$ é solução de

$$
\sum_{i=1}^{n} \phi_{i}\left(\gamma ;\left\{x_{i j}\right\}_{j=1}^{k_{i}}\right)=0
$$

onde $E\left[\phi_{i}\left(\gamma_{0} ;\left\{x_{i j}\right\}_{j=1}^{k_{i}}\right)\right]=0, i=1, \ldots, n$, sendo $\gamma_{0}$ o verdadeiro valor do parâmetro $\gamma$.

Seja $\tau=\left(\gamma^{\prime}, \theta^{\prime}\right)^{\prime}$ e

$$
\psi_{i}\left(\tau ;\left\{x_{i j}\right\}_{j=1}^{k_{i}}, y_{i}\right)=\left(\begin{array}{c}
\phi_{i}\left(\gamma ;\left\{x_{i j}\right\}_{j=1}^{k_{i}}\right) \\
U_{i}^{*}\left(\gamma / k_{i}, \theta ; \bar{x}_{i}, y_{i}\right)
\end{array}\right)
$$

onde $\sum_{i=1}^{n} U_{i}^{*}\left(\gamma / k_{i}, \theta ; \bar{x}_{i}, y_{i}\right)$ é o escore corrigido com $\bar{x}_{i}$. no lugar de $x_{i}$ e $\gamma / k_{i}$ no lugar de $\gamma$. Segue então que

$$
E\left[\psi_{i}\left(\tau_{0} ;\left\{x_{i j}\right\}_{j=1}^{k_{i}}, y_{i}\right)\right]=0
$$

onde $\tau_{0}=\left(\gamma_{0}^{\prime}, \theta_{0}^{\prime}\right)^{\prime}$.

Desta forma, podemos provar que existe $\widehat{\tau}=\left(\hat{\gamma}^{\prime}, \hat{\theta}^{\prime}\right)^{\prime}$, solução de

$$
\sum_{i=1}^{n} \psi_{i}\left(\tau ;\left\{x_{i j}\right\}_{j=1}^{k_{i}}, y_{i}\right)=0
$$


consistente e assintoticamente normal. Conseqüentemente, $\hat{\theta}$, solução de

$$
\sum_{i=1}^{n} U_{i}^{*}\left(\hat{\gamma} / k_{i}, \theta ; \bar{x}_{i}, y_{i}\right)=0
$$

onde $\hat{\gamma}$ é solução de (2.26), também o será.

Com efeito, sejam

$$
\tilde{\Lambda}_{n}(\tau)=\frac{1}{n} \sum_{i=1}^{n} E\left[-\frac{\partial \psi_{i}}{\partial \tau}\right] \quad \text { e } \quad \tilde{\Gamma}_{n}(\tau)=\frac{1}{n} \sum_{i=1}^{n} E\left[\psi_{i}(\tau) \psi_{i}(\tau)^{\prime}\right] .
$$

Note que, particionando $\widetilde{\Lambda}_{n}$ e $\widetilde{\Gamma}_{n}$ de acordo com as dimensões de $\gamma$ e $\theta$, temos que

$$
\tilde{\Lambda}_{n}=\left(\begin{array}{cc}
\bar{A}_{n} & 0 \\
\bar{B}_{n} & \bar{\Lambda}_{n}
\end{array}\right) \text {. e } \widetilde{\Gamma}_{n}=\left(\begin{array}{cc}
\bar{C}_{n} & \bar{D}_{n} \\
\bar{D}_{n}^{\prime} & \bar{\Gamma}_{n}
\end{array}\right)
$$

onde

$$
\begin{aligned}
& \bar{A}_{n}=\frac{1}{n} \sum_{i=1}^{n} E\left[-\frac{\partial \phi_{i}}{\partial \gamma}\right], \\
& \bar{B}_{n}=\frac{1}{n} \sum_{i=1}^{n} E\left[-\frac{\partial U_{i}^{*}}{\partial \gamma}\right], \\
& \bar{C}_{n}=\frac{1}{n} \sum_{i=1}^{n} E\left[\phi_{i} \phi_{i}^{\prime}\right], \\
& \bar{D}_{n}=\frac{1}{n} \sum_{i=1}^{n} E\left[\phi_{i} U_{i}^{*^{\prime}}\right],
\end{aligned}
$$

$\operatorname{com} \bar{\Lambda}_{n}$ e $\bar{\Gamma}_{n}$ definidas em (2.8) e (2.9), respectivamente.

Sob condições de regularidade análogas às formuladas na Seção 2.3.1, com $\psi_{i},-\frac{\partial \psi_{i}}{\partial \tau}$, $\tilde{\Lambda}_{n}$ e $\tilde{\Gamma}_{n}$ no lugar de $U_{i}^{*},-\frac{\partial U_{i}^{*}}{\partial \theta}, \bar{\Lambda}_{n}$ e $\bar{\Gamma}_{n}$, respectivamente, podemos enunciar o seguinte teorema sobre a distribuição assintótica de $\widehat{\theta}$.

Teorema 2.3 Seja $\hat{\tau}=\left(\widehat{\gamma}^{\prime}, \widehat{\theta}^{\prime}\right)^{\prime}$ raiz consistente de (2.27), então $\hat{\theta}$ é assintoticamente normal com média $\theta_{0}$ e matriz de covariâncias assintótica dada por $n^{-1} \tilde{\Omega}_{n}$, com

$$
\tilde{\Omega}_{n}=\bar{\Lambda}_{n}^{-1}\left\{\bar{\Gamma}_{n}-\bar{B}_{n} \bar{A}_{n}^{-1} \bar{D}_{n}-\bar{D}_{n}^{\prime} \bar{A}_{n}^{-1} \bar{B}_{n}^{\prime}+\bar{B}_{n} \bar{A}_{n}^{-1} \bar{C}_{n}\left(\bar{A}_{n}^{\prime}\right)^{-1} \bar{B}_{n}^{\prime}\right\}\left(\bar{\Lambda}_{n}^{\prime}\right)^{-1}
$$

onde todas as matrizes estão avaliadas em $\tau_{0}=\left(\gamma_{0}^{\prime}, \theta_{0}^{\prime}\right)^{\prime}$. 
Prova: Da mesma forma que foi mostrado no Teorema 2.2, temos que $\widehat{\tau}$ é assintoticamente normal com média $\tau_{0}$ e matriz de covariâncias assintótica dada por $n^{-1}\left\{\widetilde{\Lambda}_{n}\left(\tau_{0}\right)\right\}^{-1} \widetilde{\Gamma}_{n}\left(\tau_{0}\right)$ $\left\{\tilde{\Lambda}_{n}\left(\tau_{0}\right)^{\prime}\right\}^{-1}$. De modo que a matriz de covariâncias assintótica de $\hat{\theta}, n^{-1} \widetilde{\Omega}_{n}$, é dada pela submatriz $p \times p$, inferior direita.

Depois de alguma álgebra, temos que

$$
\widetilde{\Omega}_{n}=\bar{\Lambda}_{n}^{-1}\left\{\bar{\Gamma}_{n}-\bar{B}_{n} \bar{A}_{n}^{-1} \bar{D}_{n}-\bar{D}_{n}^{\prime} \bar{A}_{n}^{-1} \bar{B}_{n}^{\prime}+\bar{B}_{n} \bar{A}_{n}^{-1} \bar{C}_{n}\left(\bar{A}_{n}^{\prime}\right)^{-1} \bar{B}_{n}^{\prime}\right\}\left(\bar{\Lambda}_{n}^{\prime}\right)^{-1}
$$

Note que $\bar{A}_{n}^{-1} \bar{C}_{n}\left(\bar{A}_{n}^{\prime}\right)^{-1}$ corresponde à matriz de covariâncias assintótica de $\widehat{\gamma}$.

Considere como caso particular $\hat{\gamma}$, o estimador usual da análise de componentes de variância, ou seja, $\widehat{\gamma}$ é solução de (2.26) com

$$
\phi_{i}\left(\gamma ;\left\{x_{i j}\right\}_{j=1}^{k_{i}}\right)=\sum_{j=1}^{k_{i}} \operatorname{Vech}\left[\left(x_{i j}-\bar{x}_{i}\right)\left(x_{i j}-\bar{x}_{i}\right)^{\prime}\right]-\left(k_{i}-1\right) \widehat{\gamma}
$$

onde $\bar{x}_{i}=\frac{1}{k_{i}} \sum_{j=1}^{k_{i}} x_{i j}$

Corolário 2.1 Seja $\hat{\gamma}$ solução de (2.26) com $\phi_{i}$ dado por (2.28), então $\hat{\theta}$ é assintoticamente normal com média $\theta_{0}$ e matriz de covariâncias assintótica dada por

$$
n^{-1} \bar{\Lambda}_{n}^{-1}\left\{\bar{\Gamma}_{n}+\bar{B}_{n} \bar{A}_{n}^{-1} \bar{C}_{n}\left(\bar{A}_{n}^{\prime}\right)^{-1} \bar{B}_{n}^{\prime}\right\}\left(\bar{\Lambda}_{n}^{\prime}\right)^{-1}
$$

Prova: É sabido que para observações distribuídas normalmente, a média amostral e a matriz de covariâncias amostral são independentes. De modo que $\widehat{\Sigma}_{u}$ e, portanto, $\hat{\gamma}=$ $\operatorname{Vech}\left(\hat{\Sigma}_{u}\right)$ são independentes de todos os termos $\left(y_{i}, x_{i}\right)$; conseqüentemente $\bar{D}_{n}=0$. Assim, a matriz de covariâncias assintótica de $\theta$ é dada por (2.29).

O estimador "sanduiche" da matriz de covariâncias assintótica de $\hat{\theta}$ pode ser construído de maneira similar àquela considerada na Seção 2.3.4. Com efeito, sejam

$$
\bar{R}_{n}(\widehat{\gamma})=\frac{\sum_{i=1}^{n} d_{i} d_{i}^{\prime}}{\left[\sum_{i=1}^{n}\left(k_{i}-1\right)\right]^{2}}
$$


onde $d_{i}=\sum_{j=1}^{k_{i}} \operatorname{Vech}\left[\left(x_{i j}-\bar{x}_{i}\right)\left(x_{i j}-\bar{x}_{i} \cdot\right)^{\prime}\right]-\left(k_{i}-1\right) \hat{\gamma}$, estimador consistente da matriz de covariâncias assintt́otica de $\hat{\gamma}$, e

$$
\bar{V}_{n}(\widehat{\tau})=\frac{1}{n} \sum_{i=1}^{n}\left(-\frac{\partial U_{i}^{*}(\widehat{\tau})}{\partial \gamma}\right),
$$

estimador consistente de $\bar{B}_{n}$. Então, temos que

$$
n^{-1}\left\{\bar{I}_{n}^{*}(\widehat{\tau})\right\}^{-1}\left\{\bar{S}_{n}(\widehat{\tau})+\bar{V}_{n}(\widehat{\tau}) \bar{R}_{n}(\widehat{\gamma}) \bar{V}_{n}(\widehat{\tau})^{\prime}\right\}\left\{\bar{I}_{n}^{*}(\widehat{\tau})^{\prime}\right\}^{-1}
$$

com $\bar{S}_{n}(\widehat{\tau})$ e $\bar{I}_{n}^{*}(\widehat{\tau})$ definidas como na Seção 2.3.4, é um estimador consistente da matriz de covariâncias assintótica de $\widehat{\theta}$, dada em (2.29).

\subsection{Existência e unicidade do escore corrigido}

Dada a função escore usual $U(\theta ; Z, Y)$, consideremos o problema de encontrar $U^{*}(\theta ; X, Y)$ tal que

$$
E\left[U^{*}(\theta ; X, Y) \mid Z, Y\right]=U(\theta ; Z, Y)
$$

No que segue, assumiremos $x$ unidimensional; os resultados para $x$ vetor aleatório podem ser estendidos sem dificuldade.

No caso em que $x_{i}=z_{i}+u_{i}$, com $u_{i} \stackrel{\text { iid }}{\sim} N\left(0, \sigma_{u}^{2}\right)$, o problema de achar uma função escore corrigida, nada mais é que o problema de encontrar um estimador não viciado de uma função não linear da média de uma variável normalmente distribuída. As variáveis normais são as $x_{i}$, que condicionadas em $z_{i}$ têm distribuição $N\left(z_{i}, \sigma_{u}^{2}\right)$. Procuramos um estimador $U_{i}^{*}\left(\theta ; x_{i}, y_{i}\right)$ não viciado de $U_{i}\left(\theta ; z_{i}, y_{i}\right)$, que é uma função não linear da média $z_{i}$, ou seja,

$$
E\left[U_{i}^{*}\left(\theta ; x_{i}, y_{i}\right) \mid z_{i}, y_{i}\right]=U_{i}\left(\theta ; z_{i}, y_{i}\right), i=1, \ldots, n
$$

Para simplificar a notação, chamemos

$$
\begin{aligned}
U(\theta ; z, y) & =g(z), \\
U^{*}(\theta ; x, y) & =g^{*}(x),
\end{aligned}
$$


$\operatorname{com} x \sim N\left(z, \sigma^{2}\right)$.

Dada $g(\cdot)$, a questão é determinar condições necessárias à existência de $g^{*}(\cdot)$, tal que

$$
E\left[g^{*}(x)\right]=g(z)
$$

Este problema é estudado em Stefanski (1989).

É claro que se (2.30) vale para alguma função $g(\cdot)$, a completividade de $x$ implica que $g^{*}(\cdot)$ é única quase certamente. Uma aplicação da teoria da transformada de Fourier permite obter condições necessárias à existência de $g^{*}(\cdot)$. De fato, (2.30) implica que

$$
\int_{-\infty}^{\infty} g^{*}(x) \frac{1}{\sqrt{2 \pi} \sigma} e^{-\frac{1}{2 \sigma^{2}}(x-z)^{2}} d x=g(z)
$$

considerando $z=i \sigma^{2} t$, temos que

$$
\frac{1}{\sqrt{2 \pi} \sigma} \int_{-\infty}^{\infty} g^{*}(x) e^{-\frac{1}{2}\left(x^{2}-2 i x \sigma^{2} t-\sigma^{4} t^{2}\right)} d x=g\left(i \sigma^{2} t\right)
$$

i.e.,

$$
\frac{1}{\sqrt{2 \pi} \sigma} \int_{-\infty}^{\infty} g^{*}(x) e^{-\frac{x^{2}}{2 \sigma^{2}}} e^{i x t} d x=\sigma e^{-\frac{\sigma^{2} t^{2}}{2}} g\left(i \sigma^{2} t\right)
$$

de modo que

$$
g^{*}(x) e^{-\frac{x^{2}}{2 \sigma^{2}}}=\frac{1}{\sqrt{2 \pi}} \int_{-\infty}^{\infty} \sigma e^{-\frac{\sigma^{2} t^{2}}{2}} g\left(i \sigma^{2} t\right) e^{-i x t} d t,
$$

portanto, $g^{*}(x) e^{-\frac{x^{2}}{2 \sigma^{2}}}$ é a transformada de Fourier da função $\sigma e^{-\frac{\sigma^{2} t^{2}}{2}} g\left(i \sigma^{2} t\right)$. Para que exista a transformada é condição necessária que $g\left(i \sigma^{2} t\right)$ seja função inteira da variável complexa $t$. Além disso, é fácil ver que $\lim _{|t| \rightarrow \infty} e^{-\frac{\sigma^{2} t^{2}}{2}} g\left(i \sigma^{2} t\right)=0$ e se $\left|e^{-\frac{\sigma^{2} t^{2}}{2}} g\left(i \sigma^{2} t\right)\right|$ é integrável em $(-\infty, \infty)$, então $g^{*}(\cdot)$ é real e contínua, $e^{-\frac{x^{2}}{2 \sigma^{2}}} g^{*}(x)$ é limitada e

$$
g^{*}(x)=\int_{-\infty}^{\infty} \frac{1}{\sigma} \phi\left(\frac{t-i x}{\sigma}\right) g(-i t) d t,
$$

onde $\phi$ é a densidade da normal estandar (veja Kolmogorov e Fomin, 1979). Em alguns casos, a equação (2.31) permite encontrar $g^{*}(\cdot)$ em forma fechada. 
Por outro lado, sob certas condições, $g^{*}(\cdot)$ admite uma representação em série. De fato, como $g(\cdot)$ deve ser função inteira, temos a seguinte representação

$$
g(z)=\sum_{k=0}^{\infty} \frac{g^{(k)}(0)}{k !} z^{k} .
$$

Considere $H_{k}(x)$ o $k$-ésimo polinômio de Hermite (Cramer, 1957) e defina $P_{k}(x)=$ $\sigma^{k} H_{k}(x \mid \sigma)$. Pode ser provado que $E\left[P_{k}(x)\right]=z^{k}$. Definindo

$$
g^{*}(x)=\sum_{k=0}^{\infty} \frac{g^{(k)}(0)}{k !} P_{k}(x)
$$

e assumindo que as operações a seguir são justificáveis,

$$
\begin{aligned}
E\left[g^{*}(x)\right] & =E\left[\sum_{k=0}^{\infty} \frac{g^{(k)}(0)}{k !} P_{k}(x)\right] \\
& =\sum_{k=0}^{\infty} \frac{g^{(k)}(0)}{k !} E\left[P_{k}(x)\right] \\
& =\sum_{k=0}^{\infty} \frac{g^{(k)}(0)}{k !} z^{k} \\
& =g(z) .
\end{aligned}
$$

Contudo, na prática só um número finito de termos em (2.32) serão considerados. Quando $\sigma^{2}$ é pequeno, soluções aproximadas ao problema (2.30), com boas propriedades, podem ser obtidas considerando finitos termos em (2.32). Para maiores detalhes, veja Stefanski (1989).

\subsection{Exemplos}

A função escore corrigida pode ser facilmente determinada quando o escore usual depende de $z$ só através de exponenciais, potências e produtos dos mesmos.

Considere uma classe de modelos com função de log-verossimilhança da forma

$$
\ell(\theta ; Z, Y)=\sum_{i=1}^{n} \ell_{i}\left(\theta ; z_{i}, y_{i}\right)
$$


onde $\theta=\left(\alpha, \beta^{\prime}\right)^{\prime} \mathrm{e}$

$$
\ell(\theta ; z, y)=c_{0}(\alpha, y)+\sum_{k=1}^{2}\left\{c_{k}(\alpha, y)\left(\beta^{\prime} z\right)^{k}+d_{k}(\alpha, y) \exp \left(b_{k} \beta^{\prime} z\right)\right\}
$$

com $b_{k}$ constante.

Alguns modelos de regressão desta classe são:

- Normal linear com média $\alpha+\beta^{\prime} z$, variância $\phi$,

$$
c_{0}=-\log \sqrt{2 \pi}-\log \sqrt{\phi}-\frac{1}{2 \phi}(y-\alpha)^{2}, c_{1}=\frac{1}{\phi}(y-\alpha), c_{2}=-\frac{1}{2 \phi}, d_{1}=d_{2}=0 .
$$

- Poisson com média $\exp \left(\alpha+\beta^{\prime} z\right)$, variância $\exp \left(\alpha+\beta^{\prime} z\right)$,

$$
c_{0}=y \alpha-\log y !, c_{1}=y, c_{2}=0, d_{1}=-\exp (\alpha), b_{1}=1, d_{2}=0 .
$$

- Gama com média $\exp \left(\alpha+\beta^{\prime} z\right)$, variância $\phi \exp \left(2\left(\alpha+\beta^{\prime} z\right)\right)$,

$$
\begin{aligned}
& c_{0}=\phi^{-1} \log \left(\phi^{-1}\right)-\phi^{-1} \alpha+\left(\phi^{-1}-1\right) \log y-\log \Gamma\left(\phi^{-1}\right), c_{1}=-\phi^{-1}, c_{2}=0, \\
& d_{1}=-\phi^{-1} y \exp (-\alpha), b_{1}=-1, d_{2}=0 .
\end{aligned}
$$

- Normal inverso com média $\exp \left(\alpha+\beta^{\prime} z\right)$, variância $\phi \exp \left(3\left(\alpha+\beta^{\prime} z\right)\right)$,

$$
\begin{aligned}
& c_{0}=-\frac{1}{2} \log \left(2 \pi y^{3}\right)+\frac{1}{2} \log \phi^{-1}-\frac{\phi^{-1}}{2 y}, c_{1}=c_{2}=0, \\
& d_{1}=\phi^{-1} \exp (-\alpha), b_{1}=-1, d_{2}=-\frac{\phi^{-1} y}{2} \exp (-2 \alpha), d_{2}=-2 .
\end{aligned}
$$

- Valor extremo com média $\alpha+\beta^{\prime} z-\phi \psi(1)$ (onde $\psi(\cdot)$ é a função digama), variância $\frac{1}{6} \pi^{2} \phi^{2}$

$$
c_{0}=\log \phi^{-1}+\frac{y-\alpha}{\phi}, c_{1}=-\frac{1}{\phi}, c_{2}=0, d_{1}=\exp \left(\frac{y-\alpha}{\phi}\right), b_{1}=-\frac{1}{\phi}, d_{2}=0 .
$$

A função escore é dada por $U(\theta ; Z, Y)=\sum_{i=1}^{n} U_{i}\left(\theta ; z_{i}, y_{i}\right)$, onde

$$
U_{i}\left(\theta ; z_{i}, y_{i}\right)=\left(U_{i \alpha}\left(\theta ; z_{i}, y_{i}\right), U_{i \beta}\left(\theta ; z_{i}, y_{i}\right)^{\prime}\right)^{\prime}
$$


com

$$
\begin{aligned}
U_{i \alpha}\left(\theta ; z_{i}, y_{i}\right) & =\frac{\partial}{\partial \alpha} \ell_{i}\left(\theta ; z_{i}, y_{i}\right) \\
& =\frac{\partial}{\partial \alpha} c_{0}\left(\alpha ; y_{i}\right)+\sum_{k=1}^{2}\left\{\frac{\partial}{\partial \alpha} c_{k}\left(\alpha ; y_{i}\right)\left(\beta^{\prime} z_{i}\right)^{k}+\frac{\partial}{\partial \alpha} d_{k}\left(\alpha, y_{i}\right) \exp \left(b_{k} \beta^{\prime} z_{i}\right)\right\}
\end{aligned}
$$

e

$$
\begin{aligned}
U_{i \beta}\left(\theta ; z_{i}, y_{i}\right) & =\frac{\partial}{\partial \beta} \ell_{i}\left(\theta ; z_{i}, y_{i}\right) \\
& =\sum_{k=1}^{2}\left\{k c_{k}\left(\alpha ; y_{i}\right)\left(\beta^{\prime} z_{i}\right)^{k-1} z_{i}+b_{k} d_{k}\left(\alpha ; y_{i}\right) \exp \left(b_{k} \beta^{\prime} z_{i}\right) z_{i}\right\}
\end{aligned}
$$

Suponha $x=z+u \operatorname{com} u \sim N\left(0, \Sigma_{u}\right), \Sigma_{u}$ conhecida, de modo que $x \mid z \sim N\left(z, \Sigma_{u}\right)$.

Na determinação de $U^{*}(\theta ; X, Y)$, faremos uso das seguintes identidades, obtidas a partir da função geradora de momentos da variável $x$ :

$$
\begin{aligned}
E\left[\exp \left(\beta^{\prime} x\right) \mid z\right] & =\exp \left(\beta^{\prime} z+\xi\right) \\
E\left[x \exp \left(\beta^{\prime} x\right) \mid z\right] & =\left(z+\Sigma_{u} \beta\right) \exp \left(\beta^{\prime} z+\xi\right), \\
E\left[x x^{\prime} \exp \left(\beta^{\prime} x\right) \mid z\right] & =\left\{\Sigma_{u}+\left(z+\Sigma_{u} \beta\right)\left(z+\Sigma_{u} \beta\right)^{\prime}\right\} \exp \left(\beta^{\prime} z+\xi\right),
\end{aligned}
$$

onde $\xi=\frac{\beta^{\prime} \Sigma_{u} \beta}{2}$.

Usando estas identidades é fácil ver que o escore corrigido é dado por $U^{*}(\theta ; X, Y)=$ $\sum_{i=1}^{n} U_{i}^{*}\left(\theta ; x_{i}, y_{i}\right)$, onde $U_{i}^{*}\left(\theta ; x_{i}, y_{i}\right)=\left(U_{i \alpha}^{*}\left(\theta ; x_{i}, y_{i}\right), U_{i \beta}^{*}\left(\theta ; x_{i}, y_{i}\right)^{\prime}\right)^{\prime}$, com

$$
\begin{aligned}
U_{i \alpha}^{*}\left(\theta ; x_{i}, y_{i}\right)= & \frac{\partial}{\partial \alpha} c_{0}\left(\alpha, y_{i}\right)+\sum_{k=1}^{2}\left\{\frac{\partial}{\partial \alpha} c_{k}\left(\alpha ; y_{i}\right)\left(\beta^{\prime} x_{i}\right)^{k}+\frac{\partial}{\partial \alpha} d_{k}\left(\alpha ; y_{i}\right) \exp \left(b_{k} \beta^{\prime} x_{i}-b_{k}^{2} \xi\right)\right\} \\
& -\frac{\partial}{\partial \alpha} c_{2}\left(\alpha, y_{i}\right) \beta^{\prime} \Sigma_{u} \beta
\end{aligned}
$$

e

$$
\begin{aligned}
U_{i \beta}^{*}\left(\theta ; x_{i}, y_{i}\right)= & c_{1}\left(\alpha, y_{i}\right) x_{i}+2 c_{2}\left(\alpha ; y_{i}\right)\left(x_{i} x_{i}^{\prime}-\Sigma_{u}\right) \beta \\
& +\sum_{k=1}^{2} b_{k} d_{k}\left(\alpha, y_{i}\right)\left(x_{i}-b_{k} \Sigma_{u} \beta\right) \exp \left(b_{k} \beta^{\prime} x_{i}-b_{k}^{2} \xi\right)
\end{aligned}
$$


A seguir é apresentado um exemplo onde o escore corrigido não existe.

Considere um modelo de regressão logística simples com erros nas variáveis (veja Carroll et al., 1994, e Stefanski e Carroll, 1985), onde

$$
\operatorname{pr}(y=1 \mid z)=F(\alpha+\beta z), \quad F(t)=\frac{1}{1+e^{-t}} .
$$

A função escore é dada por

$$
U(\theta ; Z, Y)=\sum_{i=1}^{n}\left\{y_{i}-F\left(\alpha+\beta z_{i}\right)\right\}\left(1, z_{i}\right)^{\prime}
$$

Como foi visto na Seção 2.5, para encontrar o escore corrigido, se existir, é necessário estimar $F(\alpha+\beta y)$ e $z F(\alpha+\beta z)$ sem vício. Conseqüentemente, estas funções devem ser funções inteiras da variável complexa $t$. Como $F(t)=\frac{1}{1+e^{-t}}$ tem singularidades em múltiplos de $i \pi$, concluímos que não existe escore corrigido, $\forall(\alpha, \beta)$. Contudo, escores corrigidos aproximados podem ser encontrados para este modelo (veja Stefanski, 1989).

\subsection{Extensão do método quando a distribuição do er- | ro de medição é normal contaminada}

Como foi visto na Seção 2.5, a existência do escore corrigido depende fortemente da suposição de normalidade sobre o erro de medição. No entanto, é sabido que a inferência baseada na distribuição normal é vulnerável à presença de "outliers" nos dados que provêm de distribuições com caudas mais pesadas.

Nesta seção propomos o uso da distribuição normal contaminada sugerida por Tukey (1960), como alternativa à normal, por ter caudas mais pesadas e, portanto, "acomodar" possíveis "outliers" presentes nas covariáveis observadas.

Quando o erro de medição segue uma distribuição normal contaminada, escores corrigidos podem ser facilmente obtidos para modelos onde o escore usual depende de $z$ através de exponenciais e potências. Como no caso de normalidade (Seção 2.6), a chave para iden- 
tificar o escore corrigido é o uso de identidades baseadas na função geradora de momentos de uma normal contaminada.

Para simplificar a exposição, apresentamos os resultados para o caso de uma normal contaminada univariada.

Considere $x=z+u$, onde a função de densidade de $u$ é dada por

$$
f(u)=(1-\varepsilon) \phi(u)+\varepsilon \phi\left(\frac{u}{\gamma}\right),
$$

onde $\phi$ é a densidade da normal padrão, $\varepsilon$ conhecido.

Desta forma, com $\varepsilon$ pequeno (por exemplo, $\varepsilon=0,05$ ), obtemos um modelo apropriado quando a distribuição da covariável $x$ é contaminada com uma pequena fração de "outliers".

Segue que $x \mid z$ tem densidade dada por

$$
f(x \mid z)=(1-\varepsilon) \phi(x-z)+\varepsilon \phi\left(\frac{x-z}{\gamma}\right),
$$

de modo que $E[x \mid z]=z$ e $\operatorname{Var}[x \mid z]=(1-\varepsilon)+\varepsilon \gamma^{2}$.

Em princípio, suponha $\gamma$ conhecida. Para modelos com função de log-verossimilhança da forma (2.33), o escore corrigido pode ser facilmente obtido usando as seguintes identidades para a função geradora de momentos da variável $x$ :

$$
\begin{aligned}
E[\exp (\beta x) \mid z]= & (1-\varepsilon) \exp \left(\beta z+\frac{\beta^{2}}{2}\right)+\varepsilon \exp \left(\beta z+\frac{\beta^{2} \gamma^{2}}{2}\right) \\
E[x \exp (\beta x) \mid z]= & (1-\varepsilon)(z+\beta) \exp \left(\beta z+\frac{\beta^{2}}{2}\right)+\varepsilon\left(z+\beta \gamma^{2}\right) \exp \left(\beta z+\frac{\beta^{2} \gamma^{2}}{2}\right) \\
E\left[x^{2} \exp (\beta x) \mid z\right]= & (1-\varepsilon)\left[1+(z+\beta)^{2}\right] \exp \left(\beta z+\frac{\beta^{2}}{2}\right) \\
& +\varepsilon\left[\gamma^{2}+\left(z+\beta \gamma^{2}\right)^{2}\right] \exp \left(\beta z+\frac{\beta^{2} \gamma^{2}}{2}\right)
\end{aligned}
$$

Como exemplo, encontramos o escore corrigido para o modelo de regressão Poisson com média $\exp (\alpha+\beta z)$ e variância $\exp (\alpha+\beta z)$. 
A função de log-verossimilhança é dada por

$$
\ell(\theta ; Z, Y)=\sum_{i=1}^{n}\left\{-\exp \left(\alpha+\beta z_{i}\right)+y_{i}\left(\alpha+\beta z_{i}\right)-\log y_{i} !\right\}
$$

onde $\theta=(\alpha, \beta)^{\prime}$ e a função escore é $U^{*}(\theta ; Z, Y)=\left(U_{\alpha}(\theta ; Z, Y), U_{\beta}(\theta ; Z, Y)\right)^{\prime}$, com

$$
\begin{aligned}
& U_{\alpha}(\theta ; Z, Y)=\sum_{i=1}^{n}\left\{-\exp \left(\alpha+\beta z_{i}\right)+y_{i}\right\} \\
& U_{\beta}(\theta ; Z, Y)=\sum_{i=1}^{n}\left\{-\exp \left(\alpha+\beta z_{i}\right) z_{i}+y_{i} z_{i}\right\}
\end{aligned}
$$

Usando as identidades (2.37) e (2.38), é fácil ver que o escore corrigido é $U^{*}(\theta ; X, Y)=$ $\left(U_{\alpha}^{*}(\theta ; X, Y), U_{\beta}^{*}(\theta ; X, Y)\right)^{\prime}$, onde

$$
\begin{aligned}
& U_{\alpha}^{*}(\theta ; X, Y)=\sum_{i=1}^{n}\left\{-\exp \left(\alpha+\beta x_{i}\right) a^{-1}+y_{i}\right\} \\
& U_{\beta}^{*}(\theta ; X, Y)=\sum_{i=1}^{n}\left\{-\exp \left(\alpha+\beta x_{i}\right)\left[x a^{-1}-b a^{-2} \beta\right]+y_{i} x_{i}\right\}
\end{aligned}
$$

com

$$
\begin{aligned}
a & =(1-\varepsilon) \exp \left(\frac{\beta^{2}}{2}\right)+\varepsilon \exp \left(\frac{\beta^{2} \gamma^{2}}{2}\right) \\
\mathrm{e} \quad b & =(1-\varepsilon) \exp \left(\frac{\beta^{2}}{2}\right)+\varepsilon \exp \left(\frac{\beta^{2} \gamma^{2}}{2}\right) \gamma^{2}
\end{aligned}
$$

Se $\gamma$ é desconhecida, é possível obter um estimador $\widehat{\gamma}$ a partir de medições replicadas das $z_{i}$ 's. Se $x_{i j}=x_{i}+u_{i j}, i=1, \ldots, n, j=1, \ldots, k_{i}$, onde $u_{i j}$ se distribui como uma normal contaminada com média 0 e variância igual a $(1-\varepsilon)+\varepsilon \gamma^{2}$, o estimador usual da análise de componentes de variância é

$$
\hat{\gamma}^{2}=\frac{\sum_{i=1}^{n} \sum_{j=1}^{k_{i}}\left(x_{i j}-\bar{x}_{i}\right)^{2}-\sum_{i=1}^{n}\left(k_{i}-1\right)(1-\varepsilon)}{\varepsilon \sum_{i=1}^{n}\left(k_{i}-1\right)},
$$

onde $\bar{x}_{i}$. $=\frac{1}{k_{i}} \sum_{j=1}^{k_{i}} x_{i j}$, ou seja, $\hat{\gamma}^{2}$ é solução da equação $\sum_{i=1}^{n} \phi_{i}\left(\gamma ;\left\{x_{i j}\right\}_{j=1}^{k_{i}}\right)=0$, com $\phi_{i}\left(\gamma ;\left\{x_{i j}\right\}_{j=1}^{k_{i}}\right)=\sum_{j=1}^{k_{i}}\left(x_{i j}-\bar{x}_{i}\right)^{2}-\left(k_{i}-1\right)\left[(1-\varepsilon)+\varepsilon \gamma^{2}\right]$, e os resultados da Seção 2.4 se aplicam também neste caso. 


\section{Capítulo 3}

\section{Testes de hipóteses}

\subsection{Introdução}

Na literatura o estudo de testes de hipóteses em modelos com erros nas variáveis tem se concentrado na comparação de testes de associação entre uma variável resposta escalar $y$ e uma covariável $z$, em modelos lineares generalizados. Uma forma de medir a perda de eficiência quando uma estatística de teste naive $T_{X}$ é usada no lugar da estatística usual $T_{Z}$ quando $z$ é realmente observada, é pela eficiência relativa assintótica de $T_{X}$ com respeito a $T_{Z}$ para alternativas locais, que denotaremos por $\operatorname{ARE}\left(T_{X}: T_{Z}\right)$ (Cox e Hinkley, 1974).

Tosteson e Tsiatis (1988) comparam o poder local do teste escore eficiente, o teste escore "naive" e o teste obtido quando $z$ é realmente observada, para testar a hipótese de associação assumindo várias estruturas de erros de medição. Diferentemente dos testes de Wald e de razão de verossimilhança, testes escore não requerem estimadores de máxima verossimilhança irrestritos e portanto são mais facilmente obtidos. Stefanski e Carroll (1990) investigam o comportamento do teste de Wald obtido utilizando o estimador corrigido de Stefanski (1985) que apresenta um vício menor do que o estimador "naive" mais ainda é inconsistente. Considerando uma estrutura aditiva de erros de medição, o teste baseado no estimador corrigido tem o mesmo poder local do que o teste de Wald "naive". Lagakos (1988) estuda o comportamento de testes de Wald em modelos lineares e testes 
escore nos modelos de regressão logística e de riscos proporcionais de Cox, considerando diferentes estruturas de erros de medição e assumindo uma distribuição conjunta arbitrária para $z$ e $x$. Um resultado interessante, provado por Lagakos (1988) para estes tipos de testes e por Tosteson e Tsiatis (1988) para testes escore em modelos lineares generalizados com uma única covariável, estabelece que a eficiência relativa assintótica do teste "naive" com respeito ao teste obtido se $z$ fosse realmente observada, é simplesmente o quadrado do coeficiente de correlação entre $x$ e $z$.

Assumindo que o método do escore corrigido apresentado no Capítulo 2 pode ser aplicado produzindo estimadores consistentes e assintoticamente normais para os parâmetros de um modelo, testes dos tipos razão de verossimilhança, escore e Wald podem ser construídos a partir dos estimadores e sua distribuição assintótica.

Na Seção 3.2 são propostos cinco testes assintóticos para provar hipóteses de interesse baseado no método de estimação do escore corrigido.

Nas Seções 3.3 e 3.4 comparamos os testes escore e de Wald, com distribuição assintótica qui-quadrado, construídos na Seção 3.2, com os respectivos testes "naive", para modelos lineares generalizados estruturais. A aplicação da técnica do escore corrigido nos leva à consideração de uma classe particular de modelos lineares generalizados, a classe Tweedie (Jørgensen, 1987) com média log-linear, e o modelo de regressão linear normal. Para estes modelos o escore corrigido e a variância assintótica dos estimadores são facilmente obtidos. Por outro lado, também mostramos que, para essa classe de modelos, o vício assintótico do estimador "naive" pode ser obtido explicitamente.

\subsection{Testes de hipóteses baseados na função escore corrigida}

Assumindo que o método do escore corrigido apresentado no Capítulo 2 pode ser aplicado, nesta seção são propostos cinco testes assintóticos para provar hipóteses de interesse, 
baseados nas propriedades assintóticas dos estimadores, obtidas na Seção 2.3.

Considere a partição do vetor de parâmetros $\theta \in \mathbb{R}^{p} \operatorname{como} \theta=\left(\lambda^{\prime}, \psi^{\prime}\right)^{\prime}$, onde $\psi$ é o parâmetro de interesse, de dimensão $s \times 1$, e $\lambda$ é um parâmetro "nuisance", de dimensão $(p-s) \times 1$.

Suponha que dadas as observações $\left(y_{1}, x_{1}\right), \ldots,\left(y_{n}, x_{n}\right)$, queremos testar a hipótese nula $H_{0}: \psi=\psi_{0}$ na presença do parâmetro "nuisance" $\lambda$.

Assumimos que quando $n$ cresce para infinito as matrizes $\bar{\Lambda}_{n}(\theta)$ e $\bar{\Gamma}_{n}(\theta)$ definidas em (2.8) e (2.9), convergem para as matrizes positivas definidas $\Lambda(\theta)$ e $\Gamma(\theta)$, respectivamente.

Usamos a seguinte notação para as matrizes particionadas segundo as dimensões de $\lambda$ e $\psi$ :

$$
\Lambda(\theta)=\left(\begin{array}{cc}
\Lambda_{\lambda \lambda}(\theta) & \Lambda_{\lambda \psi}(\theta) \\
\Lambda_{\psi \lambda}(\theta) & \Lambda_{\psi \psi}(\theta)
\end{array}\right), \quad \Gamma(\theta)=\left(\begin{array}{cc}
\Gamma_{\lambda \lambda}(\theta) & \Gamma_{\lambda \psi}(\theta) \\
\Gamma_{\psi \lambda}(\theta) & \Gamma_{\psi \psi}(\theta)
\end{array}\right),
$$

$\operatorname{com} \Lambda_{\lambda \lambda}(\theta)$ de dimensão $(p-s) \times(p-s)$ e assim por diante.

Analogamente, a função escore corrigida $U^{*}(\theta)$ pode ser particionada como

$$
U^{*}(\theta)=\left(\begin{array}{c}
U_{\lambda}^{*}(\theta) \\
U_{\psi}^{*}(\theta)
\end{array}\right)
$$

Sejam $\hat{\theta}=\left(\hat{\lambda}^{\prime}, \widehat{\psi}^{\prime}\right)^{\prime}$ e $\widehat{\theta}_{0}=\left(\hat{\lambda}_{0}^{\prime}, \psi_{0}^{\prime}\right)^{\prime}$ estimadores consistentes de $\theta$, satisfazendo

$$
\begin{aligned}
U^{*}(\widehat{\theta}) & =0, \\
U_{\lambda}^{*}\left(\hat{\theta}_{0}\right) & =0,
\end{aligned}
$$

ou seja, $\hat{\theta}$ e $\hat{\theta}_{0}$ são os estimadores corrigidos irrestrito e restrito a $H_{0}$, respectivamente.

As seguintes estatísticas podem ser definidas:

$$
\begin{aligned}
W & =n\left(\widehat{\psi}-\psi_{0}\right)^{\prime} \hat{\Lambda}_{\psi \psi . \lambda}\left(\hat{\theta}_{0}\right)\left(\hat{\psi}-\psi_{0}\right), \\
Q & =n^{-1} U_{\psi}^{*}\left(\hat{\theta}_{0}\right)^{\prime} \hat{\Lambda}_{\psi \psi . \lambda}^{-1}\left(\hat{\theta}_{0}\right) U_{\psi}^{*}\left(\hat{\theta}_{0}\right), \\
W_{c} & =n\left(\hat{\psi}-\psi_{0}\right)^{\prime} \hat{\Omega}_{\psi \psi}^{-1}\left(\widehat{\theta}_{0}\right)\left(\widehat{\psi}-\psi_{0}\right), \\
Q_{c} & =n^{-1} U_{\psi}^{*}\left(\hat{\theta}_{0}\right)^{\prime} \hat{\Lambda}_{\psi \psi \cdot \lambda}^{-1}\left(\hat{\theta}_{0}\right) \hat{\Omega}_{\psi \psi}^{-1}\left(\hat{\theta}_{0}\right) \hat{\Lambda}_{\psi \psi . \lambda}^{-1}\left(\hat{\theta}_{0}\right) U_{\psi}^{*}\left(\hat{\theta}_{0}\right),
\end{aligned}
$$


onde $\widehat{\Lambda}_{\psi \psi . \lambda}\left(\hat{\theta}_{0}\right)$ e $\widehat{\Omega}_{\psi \psi}\left(\hat{\theta}_{0}\right)$ são estimadores consistentes das matrizes $\Lambda_{\psi \psi . \lambda}\left(\theta_{0}\right)$ e $\Omega_{\psi \psi}\left(\theta_{0}\right)$, dadas por

$$
\begin{aligned}
\Lambda_{\psi \psi . \lambda}\left(\theta_{0}\right) & =\Lambda_{\psi \psi}\left(\theta_{0}\right)-\Lambda_{\psi \lambda}\left(\theta_{0}\right) \Lambda_{\lambda \lambda}^{-1}\left(\theta_{0}\right) \Lambda_{\lambda \psi}\left(\theta_{0}\right), \\
\Omega_{\psi \psi}\left(\theta_{0}\right) & =\left(\Lambda^{-1}\left(\theta_{0}\right) \Gamma\left(\theta_{0}\right) \Lambda^{-1}\left(\theta_{0}\right)^{\prime}\right)_{\psi \psi},
\end{aligned}
$$

respectivamente.

Quando o escore corrigido corresponde ao gradiente de uma log-verossimilhança corrigida $\ell^{*}(\theta)$, isto é,

$$
U^{*}(\theta)=\frac{\partial \ell^{*}(\theta)}{\partial \theta}
$$

é possível definir um teste de razão de verossimilhança corrigida. Nesse caso, $\hat{\theta}$ maximiza globalmente $\ell^{*}(\theta)$ e $\widehat{\theta}_{0}$ maximiza $\ell^{*}(\theta)$ sob $H_{0}$.

A estatística da razão de verossimilhança corrigida é definida por

$$
L=2\left\{\ell^{*}(\widehat{\theta})-\ell^{*}\left(\widehat{\theta}_{0}\right)\right\}
$$

As estatísticas $W, Q$ e $L$ têm a mesma distribuição assintótica sob a hipótese nula. $W_{c}$ e $Q_{c}$ também são assintoticamente equivalentes. Esses resultados são apresentados no seguinte teorema.

Teorema 3.1 Se as condições do Teorema 2.2 são satisfeitas, então:

$$
W \stackrel{\mathcal{D}}{\longrightarrow} \sum_{i=1}^{s} \mu_{i} V_{i}
$$

onde $V_{1}, \ldots, V_{s}$ são variáveis aleatórias independentes com distribuição $\chi_{1}^{2}$ e $\mu_{1}, \ldots, \mu_{s}$ são os autovalores da matriz

$$
\Lambda_{\psi \psi . \lambda}\left(\theta_{0}\right) \Omega_{\psi \psi}\left(\theta_{0}\right)
$$

Além disso, $W$ é assintoticamente equivalente a $Q$, e a $L$ no caso em que (3.9) é válido.

$$
W_{c} \stackrel{\mathcal{D}}{\longrightarrow} \chi_{s}^{2}
$$

com $Q_{c}$ assintoticamente equivalente $a W_{c}$. 
Prova: Sejam $W^{*}, Q^{*}, W_{c}^{*}$ e $Q_{c}^{*}$ definidas como $W, Q, W_{c}$ e $Q_{c}$, respectivamente, com $\Lambda_{\psi \psi . \lambda}\left(\theta_{0}\right)$ e $\Omega_{\psi \psi}\left(\theta_{0}\right)$ no lugar dos estimadores $\hat{\Lambda}_{\psi \psi}\left(\hat{\theta}_{0}\right)$ e $\hat{\Omega}_{\psi \psi}\left(\hat{\theta}_{0}\right)$.

Se mostrarmos que, sob $H_{0}, W^{*}, Q^{*}, L \stackrel{\mathcal{D}}{\longrightarrow} \sum_{i=1}^{n} \mu_{i} V_{i}$ e $W_{c}^{*}, Q_{c}^{*} \stackrel{\mathcal{D}}{\longrightarrow} \chi_{s}^{2}$, estaremos provando (a) e (b) do teorema, respectivamente. De fato, se $W^{*} \stackrel{\mathcal{D}}{\longrightarrow} \sum_{i=1}^{n} \mu_{i} V_{i}$, chamando $v=\sqrt{n}\left(\widehat{\psi}-\psi_{0}\right)$, temos que

$$
\frac{W}{W^{*}}=\frac{v^{\prime} \widehat{\Lambda}_{\psi \psi \cdot \lambda}\left(\widehat{\theta}_{0}\right) v}{v^{\prime} \Lambda_{\psi \psi \cdot \lambda}\left(\theta_{0}\right) v} .
$$

De modo que, usando o Teorema de Courant (veja, por exemplo, Sen e Singer, 1993), segue que

$$
\lambda_{s} \leq \frac{W}{W^{*}} \leq \lambda_{1}
$$

onde $\lambda_{1}$ e $\lambda_{s}$ representam o primeiro e o $s$-ésimo autovalor da matriz $\widehat{\Lambda}_{\psi \psi \cdot \lambda}\left(\hat{\theta}_{0}\right) \Lambda_{\psi \psi \cdot \lambda}\left(\theta_{0}\right)$. Como $\widehat{\Lambda}_{\psi \psi \cdot \lambda}\left(\hat{\theta}_{0}\right) \stackrel{\mathrm{p}}{\longrightarrow} \Lambda_{\psi \psi \cdot \lambda}\left(\theta_{0}\right)$, temos que $\widehat{\Lambda}_{\psi \psi \cdot \lambda}\left(\hat{\theta}_{0}\right) \Lambda_{\psi \psi \cdot \lambda}^{-1}\left(\theta_{0}\right) \stackrel{\mathrm{p}}{\longrightarrow} I_{s}$ e, conseqüentemente, $\lambda_{1} \stackrel{\mathrm{p}}{\longrightarrow} 1 \mathrm{e} \lambda_{s} \stackrel{\mathrm{p}}{\longrightarrow} 1$. Portanto, $\stackrel{W}{W^{*}} \stackrel{\mathrm{p}}{\longrightarrow} 1 \mathrm{e}$, escrevendo $W=W^{*} \frac{W}{W^{*}}$, concluímos pelo Teorema de Slutsky que $W \stackrel{\mathcal{D}}{\longrightarrow} \sum_{i=1}^{s} \mu_{i} V_{i}$. Analogamente, provamos que $Q^{*}, W_{c}^{*}$ e $Q_{c}^{*}$ são assintoticamente equivalentes a $Q, W_{c}$ e $Q_{c}$, respectivamente.

Provaremos a seguir (a).

Uma expansão de $U^{*}\left(\theta_{0}\right)$ em torno do ponto $\hat{\theta}$ produz

$$
\frac{1}{\sqrt{n}} U^{*}\left(\theta_{0}\right)=\frac{1}{\sqrt{n}} U^{*}(\widehat{\theta})+\bar{I}_{n}^{*}(\tilde{\theta}) \sqrt{n}\left(\hat{\theta}-\theta_{0}\right),
$$

onde $\tilde{\theta}$ é tal que $\left\|\tilde{\theta}-\theta_{0}\right\|<\left\|\widehat{\theta}-\theta_{0}\right\|$.

A partir do Teorema 2.2, temos que

$$
\sqrt{n}\left(\hat{\theta}-\theta_{0}\right)=O_{p}(1)
$$

de modo que, de (3.1), (3.12), (3.13) e (2.16), segue que

$$
\frac{1}{\sqrt{n}} U^{*}\left(\theta_{0}\right)=\Lambda\left(\theta_{0}\right) \sqrt{n}\left(\hat{\theta}-\theta_{0}\right)+o_{p}(1) \text {. }
$$

Analogamente, uma expansão de $U^{*}\left(\theta_{0}\right)$ em torno do ponto $\hat{\theta}_{0}$ produz

$$
\frac{1}{\sqrt{n}} U^{*}\left(\theta_{0}\right)=\frac{1}{\sqrt{n}} U^{*}\left(\hat{\theta}_{0}\right)+\Lambda\left(\theta_{0}\right) \sqrt{n}\left(\hat{\theta}_{0}-\theta_{0}\right)+o_{p}(1) .
$$


Das expansões (3.14) e (3.15), é possivel obter a seguinte relação entre $\hat{\lambda}_{0}$ e $\hat{\lambda}$ :

$$
\hat{\lambda}_{0}=\hat{\lambda}+\Lambda_{\lambda \lambda}^{-1}\left(\theta_{0}\right) \Lambda_{\lambda \psi}\left(\theta_{0}\right)\left(\widehat{\psi}-\psi_{0}\right)
$$

(veja, por exemplo, Cox e Hinkley, 1974). Assim, de (3.2), (3.14), (3.15) e (3.16), temos que

$$
\begin{aligned}
& \sqrt{n}\left[\Lambda_{\psi \psi}\left(\theta_{0}\right)-\Lambda_{\psi \lambda}\left(\theta_{0}\right) \Lambda_{\lambda \lambda}^{-1}\left(\theta_{0}\right) \Lambda_{\lambda \psi}\left(\theta_{0}\right)\right]\left(\widehat{\psi}-\psi_{0}\right) \\
& =\frac{1}{\sqrt{n}} U_{\psi}^{*}\left(\hat{\theta}_{0}\right)+o_{p}(1)
\end{aligned}
$$

ou seja,

$$
\sqrt{n}\left(\widehat{\psi}-\psi_{0}\right)=\frac{1}{\sqrt{n}} \Lambda_{\psi \psi . \lambda}^{-1}\left(\theta_{0}\right) U_{\psi}^{*}\left(\hat{\theta}_{0}\right)+o_{p}(1),
$$

$\operatorname{com} \Lambda_{\psi \psi \cdot \lambda}\left(\theta_{0}\right)$ definida em (3.7).

Substituindo (3.17) na expressão para $W^{*}$, temos

$$
W^{*}=n^{-1} U_{\psi}^{*}\left(\widehat{\theta}_{0}\right)^{\prime} \Lambda_{\psi \psi \cdot \lambda}^{-1}\left(\theta_{0}\right) U_{\psi}^{*}\left(\hat{\theta}_{0}\right)+o_{p}(1)
$$

ou seja,

$$
W^{*}=Q^{*}+o_{p}(1)
$$

de modo que $W^{*}$ e $Q^{*}$ resultam assintoticamente equivalentes.

Se (3.9) for válida, expandindo em série de Taylor a função $\ell^{*}\left(\theta_{0}\right)$ em torno do ponto $\widehat{\theta}$, obtemos

$$
2\left\{\ell^{*}\left(\theta_{0}\right)-\ell^{*}(\widehat{\theta})\right\}=-n\left(\hat{\theta}-\theta_{0}\right)^{\prime} \bar{T}_{n}^{*}(\tilde{\theta})\left(\hat{\theta}-\theta_{0}\right),
$$

onde $\tilde{\theta}$ é tal que $\left\|\widetilde{\theta}-\theta_{0}\right\|<\left\|\widehat{\theta}-\theta_{0}\right\|$.

De (3.13) e (2.16), temos que

$$
2\left\{\ell^{*}\left(\theta_{0}\right)-\ell^{*}(\widehat{\theta})\right\}=-n\left(\widehat{\theta}-\theta_{0}\right)^{\prime} \Lambda\left(\theta_{0}\right)\left(\widehat{\theta}-\theta_{0}\right)+o_{p}(1) .
$$

De forma análoga, obtemos

$$
\begin{aligned}
2\left\{\ell^{*}\left(\theta_{0}\right)-\ell^{*}\left(\hat{\theta}_{0}\right)\right\} & =-n\left(\hat{\theta}_{0}-\theta_{0}\right)^{\prime} \Lambda\left(\theta_{0}\right)\left(\hat{\theta}_{0}-\theta_{0}\right)+o_{p}(1) \\
& =-n\left(\hat{\lambda}_{0}-\lambda_{0}\right)^{\prime} \Lambda_{\lambda \lambda}\left(\theta_{0}\right)\left(\hat{\lambda}_{0}-\lambda_{0}\right)+o_{p}(1),
\end{aligned}
$$


onde a última igualdade segue da definição de $\widehat{\theta}_{0}=\left(\widehat{\lambda}_{0}^{\prime}, \psi_{0}^{\prime}\right)^{\prime}$. Portanto, a partir de (3.18) e (3.19), temos que

$$
\begin{aligned}
L & =2\left\{\ell^{*}(\hat{\theta})-\ell^{*}\left(\hat{\theta}_{0}\right)\right\} \\
& =n\left(\hat{\theta}-\theta_{0}\right)^{\prime} \Lambda\left(\theta_{0}\right)\left(\hat{\theta}-\theta_{0}\right)-n\left(\hat{\lambda}_{0}-\lambda_{0}\right)^{\prime} \Lambda_{\lambda \lambda}\left(\theta_{0}\right)\left(\hat{\lambda}_{0}-\lambda_{0}\right)+o_{p}(1)
\end{aligned}
$$

Substituindo (3.16) em (3.20) e levando em conta o fato de que a matriz $\Lambda\left(\theta_{0}\right)$ é simétrica quando (3.9) é válida, isto é, $\Lambda_{\lambda \psi}=\Lambda_{\psi \lambda}^{\prime}$, temos que

$$
L=n\left(\widehat{\psi}-\psi_{0}\right)^{\prime} \Lambda_{\psi \psi \cdot \lambda}\left(\theta_{0}\right)\left(\widehat{\psi}-\psi_{0}\right)+o_{p}(1)
$$

ou seja,

$$
L=W^{*}+o_{p}(1)
$$

de modo que $L$ e $W^{*}$ resultam assintoticamente equivalentes.

Para encontrar a distribuição assintótica de $W^{*}, Q^{*}$ e $L$, é mais fácil trabalhar com $W^{*}$. De fato, a partir do Teorema 2.2 , tem-se

$$
\sqrt{n}\left(\hat{\theta}-\theta_{0}\right) \stackrel{\mathcal{D}}{\longrightarrow} N_{p}\left(0, \Omega\left(\theta_{0}\right)\right)
$$

onde $\Omega\left(\theta_{0}\right)=\Lambda^{-1}\left(\theta_{0}\right) \Gamma\left(\theta_{0}\right) \Lambda^{-1}\left(\theta_{0}\right)$ e, conseqüentemente,

$$
\sqrt{n}\left(\hat{\psi}-\psi_{0}\right) \stackrel{\mathcal{D}}{\longrightarrow} N_{s}\left(0, \Omega_{\psi \psi}\left(\theta_{0}\right)\right)
$$

portanto, $W^{*} \stackrel{\mathcal{D}}{\longrightarrow} \chi_{(k)}^{2}$, onde $k$ é o parâmetro de não centralidade dado por

$$
k=\operatorname{traço}\left\{\Lambda_{\psi \psi \cdot \lambda}\left(\theta_{0}\right) \Omega_{\psi \psi}\left(\theta_{0}\right)\right\} .
$$

Usando propriedades da distribuição qui-quadrado não central segue que

$$
W^{*} \stackrel{\mathcal{D}}{\longrightarrow} \sum_{i=1}^{s} \mu_{i} V_{i}
$$

onde $V_{1}, \ldots, V_{s}$ são variáveis aleatórias independentes com distribuição $\chi_{1}^{2}$ e $\mu_{1}, \ldots, \mu_{s}$ são os autovalores da matriz $\Lambda_{\psi \psi \cdot \lambda}\left(\theta_{0}\right) \Omega_{\psi \psi}\left(\theta_{0}\right)$ (veja, por exemplo, Rao, 1973). Isto conclui a prova de a). 
Para a prova de (b) temos, a partir de (3.21), que

$$
W_{c}^{*}=n\left(\widehat{\psi}-\psi_{0}\right) \Omega_{\psi \psi}^{-1}\left(\theta_{0}\right)\left(\widehat{\psi}-\psi_{0}\right)
$$

tem distribuição assintótica $\chi_{s}^{2}$, sob $H_{0}$. Usando (3.17) é fácil ver que $Q_{c}^{*}$ é assintoticamente equivalente a $W_{c}^{*}$.

\section{Observações}

(1) Se a matriz dada em (3.11) é igual à matriz identidade, então $W \stackrel{\mathcal{D}}{\longrightarrow} \chi_{s}^{2}$, como no caso da estatística de Wald clássica. Portanto, se o produto $\Lambda \Omega$ é aproximadamente igual à identidade, a distribuição assintótica de $L, W$ e $Q$ pode ser aproximada por uma distribuição $\chi_{s}^{2}$.

(2) Se a matriz $\Lambda$ é diagonal em blocos, isto é, $\Lambda_{\psi \lambda}=0$, então a matriz dada em (3.11) é igual a $\Gamma_{\psi \psi} \Lambda_{\psi \psi}^{-1}$. Em particular, $\Gamma_{\psi \psi} \Lambda_{\psi \psi}^{-1}$ é apropriada quando $p-s=0$, ou seja, quando não existem parâmetros "nuisance".

(3) Se $\Lambda_{\psi \lambda}=0$ e $s=1$, então a matriz dada em (3.11) é igual à razão $\Gamma_{\psi \psi} / \Lambda_{\psi \psi}$, que atua como um fator de correção da distribuição $\chi_{1}^{2}$.

(4) Estimadores consistentes das matrizes $\Lambda\left(\theta_{0}\right)$ e $\Gamma\left(\theta_{0}\right)$ foram considerados na Seção 2.3.4. Qualquer estimador consistente de $\theta_{0}$ poderia ser usado no lugar de $\hat{\theta}_{0}$. Contudo, $\widehat{\theta}_{0}$ é o estimador mais simples, especialmente quando usamos as estatísticas escore $Q$ e $Q_{c}$, pois o modelo paramétrico só precisa ser ajustado sob a hipótese nula. Na prática também será necessário calcular os quantis da distribuição de $\sum_{i=1}^{s} \widehat{\mu}_{i} V_{i}$, onde $\widehat{\mu}_{i}$ são os autovalores do estimador da matriz $\Lambda_{\psi \psi \cdot \lambda}\left(\theta_{0}\right) \Omega_{\psi \psi}\left(\theta_{0}\right)$. Se $s>1$, podem ser usados os algoritmos dados por Marazzi (1980) e Griffths e Hill (1985). Outra forma seria simular a partir da distribuição de $\sum_{i=1}^{s} \mu_{i} V_{i}$, pois variáveis com distribuição qui-quadrado podem ser facilmente simuladas. Embora a 
distribuição de $\sum_{i=1}^{s} \mu_{i} V_{i}$ seja não trivial, poderia ser aproximada por $\widehat{\mu} \chi_{s}^{2}$, onde $\widehat{\mu}=\operatorname{traço} \frac{\left(\widehat{\Lambda}_{\psi \psi . \lambda}\left(\widehat{\theta}_{0}\right) \widehat{\Omega}_{\psi \psi}\left(\widehat{\theta}_{0}\right)\right)}{s}$ (veja, por exemplo, Rao e Scott, 1981).

(5) Quando a matriz de covariâncias do erro $\Sigma_{u}$ é desconhecida, os resultados assintóticos obtidos na Seção 2.4 devem ser considerados para a construção das estatísticas dos testes.

(6) Note que se $s=1, \mu_{1}=\Lambda_{\psi \psi \cdot \lambda}\left(\theta_{0}\right) \Omega_{\psi \psi}\left(\theta_{0}\right)$, de modo que

$$
\frac{W}{\widehat{\mu}_{1}}=\frac{n\left(\hat{\psi}-\psi_{0}\right)^{2}}{\widehat{\Omega}_{\psi \psi}\left(\hat{\theta}_{0}\right)}=W_{c} \quad \text { e } \quad \frac{Q}{\widehat{\mu}_{1}}=\frac{n^{-1} U_{\psi}^{*}\left(\hat{\theta}_{0}\right)^{2}}{\widehat{\Lambda}_{\psi \psi . \lambda}\left(\hat{\theta}_{0}\right) \hat{\Omega}_{\psi \psi}\left(\hat{\theta}_{0}\right)}=Q_{c} .
$$

Pqrtanto, $\underset{\hat{\mu}_{1}}{W}=W_{c}$ e $\frac{Q}{\widehat{\mu}_{1}}=Q_{c}$, com distribuição assintótica $\chi_{1}^{2}$.

(7) O comportamento assintótico da estatística da razão de verossimilhança corrigida é similar ao encontrado para a estatística da razão de verossimilhança usual, quando um modelo incorreto é usado na construção do teste (veja Foutz e Srivastava, 1977, Royall, 1986, Rotnitzky e Jewell, 1990).

\subsubsection{Um estudo de simulação}

Uma simulação de Monte Carlo foi realizada para comparar o poder e o tamanho empíricos dos testes $L, W$ e $Q$ definidos em (3.10), (3.3) e (3.4), respectivamente.

O estudo é baseado num modelo de regressão exponencial.

A verdadeira covariável $z_{i}$ foi gerada como uma normal padrão, i.e., $z_{i} \sim N(0,1)$.

A covariável observada $x_{i}$ foi gerada condicionada na verdadeira covariável $z_{i}$, segundo o modelo $x_{i}=z_{i}+u_{i}$, onde $u_{i} \sim N\left(0, \sigma_{u}^{2}\right)$.

A variável $y_{i}$, condicionada em $z_{i}$ foi gerada como uma variável aleatória exponencial com parâmetro $\exp \left(\alpha+\beta z_{i}\right)$, com $\alpha=0$. A hipótese nula de interesse foi $\beta=0$.

1.000 replicações foram geradas para cada simulação, para o tamanho amostral $n=20$ e para valores de $\sigma_{u}^{2}$ iguais a $0.0,0.1,0.3$ e 0.5 . 
As simulações foram feitas no Laboratório de Computação Científica Avançada (LCCA) da USP, utilizando programas em FORTRAN e sub-rutinas do IMSL.

Note que, de acordo com a observação (6) da seção anterior, temos que $\frac{W}{\widehat{\mu}_{1}}=W_{c}$ e $\frac{Q}{\widehat{\mu}_{1}}=Q_{c}$.

Na Tabela 3.1 apresentamos os níveis empíricos dos testes para um nível nominal de $5 \%$. Ainda para uma amostra pequena $(n=20)$ os tamanhos empíricos dos testes de razão de verossimilhança e escore estão próximos dos valores nominais. O mesmo não acontece com o teste de Wald.

Tabela 3.1: Tamanhos empíricos dos testes para um nível nominal de $5 \%$.

\begin{tabular}{c|ccc}
$\sigma_{u}^{2}$ & $L$ & $W$ & $Q$ \\
\hline 0.0 & 5.6 & 12.0 & 6.6 \\
0.1 & 5.7 & 12.2 & 6.9 \\
0.3 & 5.6 & 12.6 & 6.8 \\
0.5 & 5.4 & 10.4 & 6.4
\end{tabular}

Um ajuste foi feito usando as distribuições das estatísticas, de forma que o tamanho empírico corresponda a 5\%. Na Figura 3.1 a), b), c) e d) apresentamos a curva de poder dos testes para valores de $\sigma_{u}^{2}$ iguais a $0.0,0.1,0.3$ e 0.5 , respectivamente. Observamos que o teste de Wald perde mais poder que os outros quando a variância do erro cresce. $\mathrm{O}$ teste de razão de verossimilhança tem um melhor comportamento que o teste escore. 
a)

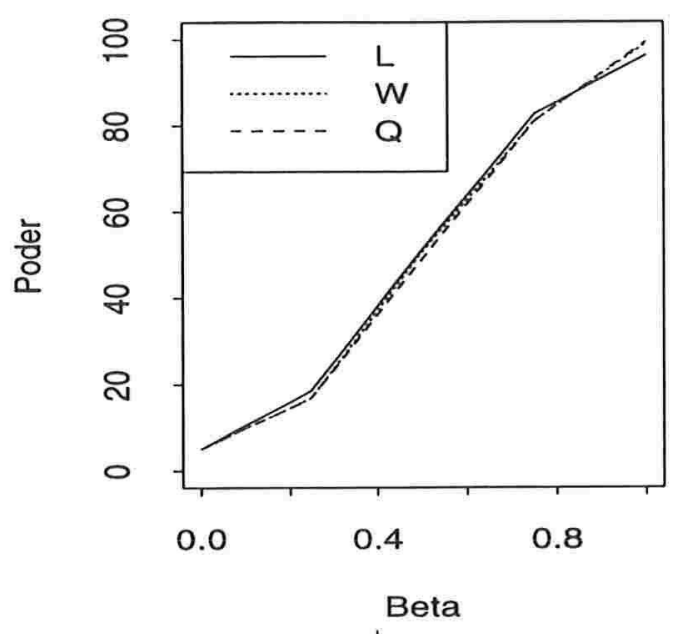

c)

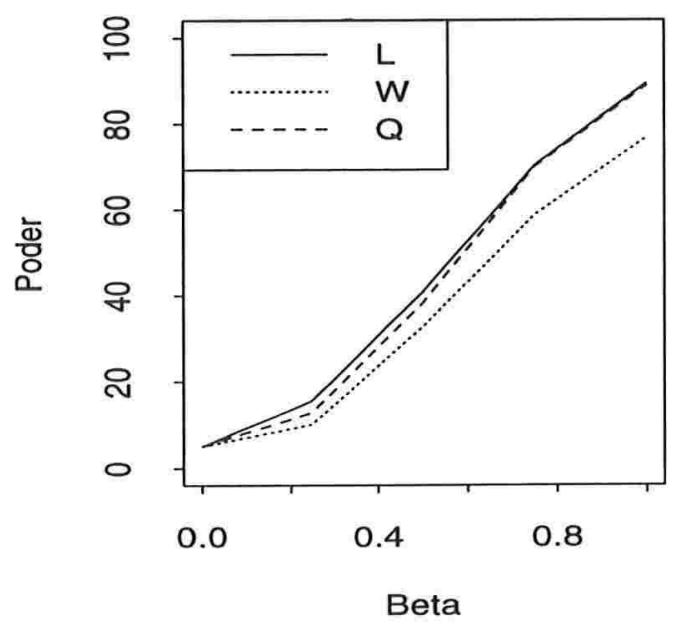

b)

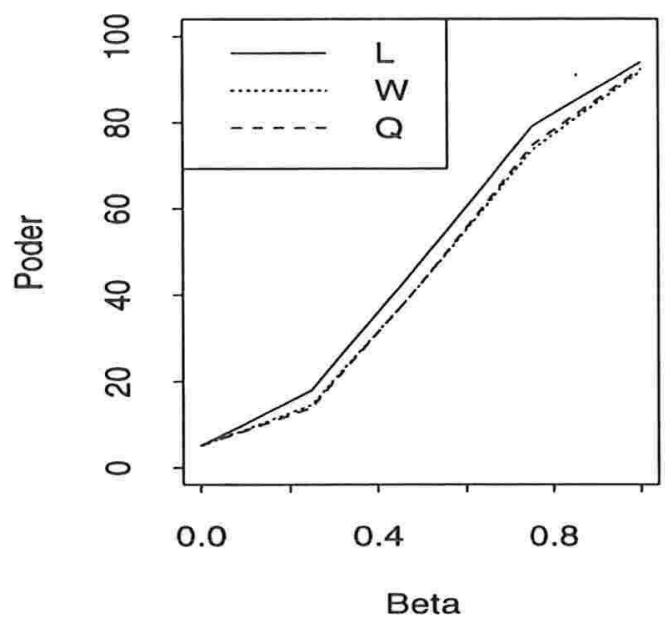

d)

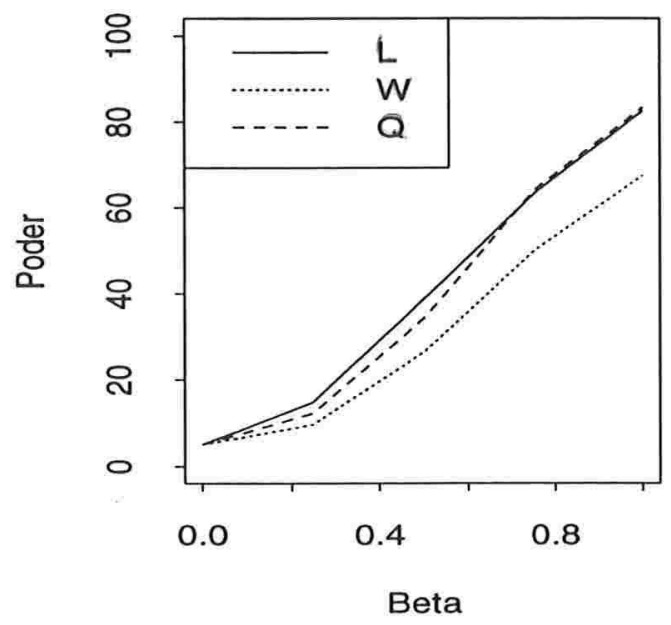

Figura 3.1: Curvas de poder dos testes $L, W$ e $Q$. a) $\sigma_{u}^{2}=0.0$, b) $\sigma_{u}^{2}=0.1$, c) $\sigma_{u}^{2}=0.3$, d) $\sigma_{u}^{2}=0.5$. 


\subsection{Comparação de testes escore}

Diferentemente dos testes de Wald e da razão de verossimilhança, os testes escore não requerem estimadores de máxima verossimilhança irrestritos e portanto são mais fácéĺs de serem obtidos. Como veremos nesta seção, existem situações onde o uso de testes escore "naive" pode ser justificado.

Considere um modelo onde $y$ é uma variável resposta escalar e $z$ é o vetor das verdadeiras covariáveis. Assumiremos

(i) y está relacionada com $z$ através de um modelo linear generalizado (McCullagh e Nelder, 1989) com $E[y \mid z]=\mu\left(\alpha+\beta^{\prime} z\right)$ e $\operatorname{Var}[y \mid z]=\phi V(\mu)$, onde $\mu(\cdot)$ é uma função monótona, $\theta=\left(\alpha, \beta^{\prime}\right)^{\prime}$ é o vetor de parâmetros desconhecido de dimensão $p=s+1$ e $\phi>0$ é o parâmetro de dispersão conhecido. A densidade condicional de $y$ dado $z, f(y \mid z)$, pertence à família exponencial uniparamétrica.

(ii) No lugar de $z$, um vetor $x$ é observado com a propriedade que

$$
f(y \mid z, x)=f(y \mid z)
$$

isto é, $x$ é condicionalmente independente da resposta $y$, dado $z$. Em outras palavras, uma vez que o valor de $z$ foi observado, nenhuma informação adicional sobre $y$ é obtida observando-se $x$.

As verdadeiras covariáveis $z$ são vetores aleatórios com média $\mu$ e matriz de covariâncias $\Sigma_{z}$.

O interesse é testar a associação entre $y$ e $z$, ou equivalentemente testar a hipótese $H_{0}: \beta=0$, na presença do parâmetro "nuisance" $\alpha$. Na notação da Seção 3.2 seria $\psi=\beta$ $\mathrm{e} \lambda=\alpha$.

Se as verdadeiras covariáveis $\left\{z_{i}\right\}_{i=1}^{n}$ fossem observadas, a estatística escore eficiente (Rao, 1973) seria baseada na densidade $f(y \mid z)$. Quando as $\left\{z_{i}\right\}_{i=1}^{n}$ não podem ser obser- 
vadas a estatística escore eficiente é baseada na densidade $f(y \mid x)$. Sob as suposições (i) e (ii), Tosteson e Tsiatis (1988) comparam o poder local do teste escore eficiente com o do teste "naive" e do teste que seria obtido se as verdadeiras covariáveis fossem realmente observadas.

Note que a suposição (ii) permite considerar várias estruturas de erros de medição. Em particular, em Tosteson e Tsiatis (1988) o interesse é considerar as covariáveis observadas como valores fixos. Este tipo de estrutura para o erro de medição é aplicável especialmente em estudos de laboratório. Como um exemplo, considere um experimento com herbicidas em que uma quantidade $x$ de herbicida foi aplicada numa planta. No entanto, a quantidade real $z$ absorvida pela planta é diferente de $x$, devido a erros de aplicação e ao próprio processo de absorção, Portanto, a concentração medida $x$ é fixada pelo planejamento do experimento e a verdadeira concentração $z$ flutua aleatoriamente em torno da concentração prefixada. Berkson (1950) descreve experimentos deste tipo.

Nesta seção o interesse é comparar o comportamento dos testes considerados em Tosteson e Tsiatis (1988), com o do teste escore corrigido $Q_{c}$, definido em (3.6), para uma classe particular de modelos lineares generalizados, onde o escore corrigido possa ser encontrado (lembremos que o escore corrigido pode não existir para alguns modelos - veja Seção 2.6). Além disso, o método do escore corrigido depende fortemente da suposição de uma estrutura aditiva de erros de medição, onde o erro é normalmente distribuído. Conseqüentemente, as seguintes suposições serão requeridas, além de (i) e (ii),

(iii) Considere $\mu\left(\alpha+\beta^{\prime} z\right)=\exp \left(\alpha+\beta^{\prime} z\right)$ e $V(\mu)=\mu^{k}, k \in \mathbb{R} \backslash(0,1)$. Estas suposições descrevem a família de modelos Tweedie (veja Jørgensen, 1987, para uma discussão sobre esta classe de modelos), com média log-linear, que resumimos na seguinte tabela: 


\begin{tabular}{ll}
\hline Distribuição & $k$ \\
\hline Estável extrema & $k<0$ \\
Normal & $k=0$ \\
Poisson & $k=1$ \\
Poisson composta & $1<k<2$ \\
Gama & $k=2$ \\
Estável positiva & $2<k<3$ \\
Normal inversa & $k=3$ \\
Estável positiva & $k>3$ \\
\hline
\end{tabular}

Na Seção 3.3.2 encontramos o escore corrigido para esta classe de modelos.

(iv) Considere uma estrutura aditiva de erros de medição, isto é,

$$
x=z+u
$$

onde $u$ é um erro de medição independente de $z$ e $y$, com média 0 e matriz de covariâncias $\Sigma_{u}$.

(v) $u \sim N\left(0, \Sigma_{u}\right), \Sigma_{u}$ conhecida ou estimada.

Dado que o objetivo é comparar o comportamento assintótico do teste escore corrigido $Q_{c}$, cuja construção depende da suposição (iv), com o dos testes escore eficiente e "naive", na Seção 3.3.1 apresentamos os resultados de Tosteson e Tsiatis (1988) sob as suposições (i), (ii) e (iv). Ou seja, diferentemente de Tosteson e Tsiatis (1988), nosso interesse é considerar $z$ e conseqüentemente $x$ variáveis aleatórias. Na Seção 3.3.2 o teste escore corrigido $Q_{c}$ é obtido sob as suposições (i) - (v).

\subsubsection{Testes escore eficiente e "naive"}

Quando as verdadeiras covariáveis $\left\{z_{i}\right\}_{i=1}^{n}$ são observadas, a estatística escore eficiente (Rao, 1973) para testar $H_{0}: \beta=0$, com $\alpha$ como parâmetro "nuisance" é baseada na densidade $f\left(y_{i} \mid z_{i}\right)$. Seja $\ell(\theta)=\sum_{i=1}^{n} \ell_{i}(\theta)=\sum_{i=1}^{n} \log f\left(y_{i} \mid z_{i}\right)$ a função de log-verossimilhança. 
Da teoria de modelos lineares generalizados (McCullagh e Nelder, 1989) tem-se que a função escore é dada por $U(\theta)=\sum_{i=1}^{n} U_{i}(\theta)$, com

$$
U_{i}(\theta)=\frac{\partial \ell_{i}(\theta)}{\partial \theta}=\left(\frac{\partial \ell_{i}(\theta)}{\partial \alpha}, \frac{\partial \ell_{i}(\theta)}{\partial \beta^{\prime}}\right)^{\prime}=\left(U_{i \alpha}(\theta), U_{i \beta}(\theta)^{\prime}\right)^{\prime},
$$

onde

$$
\begin{aligned}
& U_{i \alpha}(\theta)=q_{i}\left(\alpha+\beta^{\prime} z_{i}\right) \\
& U_{i \beta}(\theta)=q_{i}\left(\alpha+\beta^{\prime} z_{i}\right) z_{i},
\end{aligned}
$$

sendo

$$
q_{i}\left(\alpha+\beta^{\prime} z_{i}\right)=\frac{1}{\phi} \dot{\mu}\left(\alpha+\beta^{\prime} z_{i}\right) V^{-1}\left\{\mu\left(\alpha+\beta^{\prime} z_{i}\right)\right\}\left\{y_{i}-\mu\left(\alpha+\beta^{\prime} z_{i}\right)\right\}
$$

com $\dot{\mu}(\cdot)$ denotando a primeira derivada de $\mu(\cdot)$.

Assim, a estatística escore eficiente é baseada em

$$
T_{Z}=\sum_{i=1}^{n} U_{i \beta}\left(\widehat{\theta}_{0}\right)=\sum_{i=1}^{n} q_{i}\left(\widehat{\alpha}_{0}\right) z_{i}
$$

onde $\hat{\theta}_{0}=\left(\hat{\alpha}_{0}, 0^{\prime}\right)^{\prime}$, com $\hat{\alpha}_{0}$ satisfazendo

$$
\sum_{i=1}^{n} U_{i \alpha}\left(\widehat{\theta}_{0}\right)=\sum_{i=1}^{n} q_{i}\left(\widehat{\alpha}_{0}\right)=0
$$

Se as $\left\{z_{i}\right\}_{i=1}^{n}$ não podem ser observadas, a estatística escore eficiente é baseada na função de densidade marginal

$$
\begin{aligned}
f\left(y_{i} \mid x_{i}\right) & =\int f\left(y_{i}, z_{i} \mid x_{i}\right) d z_{i}=\int f\left(y_{i} \mid z_{i}\right) f\left(z_{i} \mid x_{i}\right) d z_{i} \\
& =E_{x_{i}}\left[f\left(y_{i} \mid z_{i}\right)\right]
\end{aligned}
$$

onde a segunda igualdade segue da suposição (ii) e $E_{x_{i}}[\cdot]$ denota a esperança com respeito à densidade $f\left(z_{i} \mid x_{i}\right)$.

Sejam

$$
\tilde{\ell}_{i}(\theta)=\log f\left(y_{i} \mid x_{i}\right)
$$


e

$$
\tilde{U}_{i}(\theta)=\frac{\partial \tilde{\ell}_{i}(\theta)}{\partial \theta}=\left(\frac{\partial \tilde{\ell}_{i}(\theta)}{\partial \alpha}, \frac{\partial \tilde{\ell}_{i}(\theta)}{\partial \beta^{\prime}}\right)^{\prime}=\left(\tilde{U}_{i \alpha}(\theta), \tilde{U}_{i \beta}(\theta)^{\prime}\right)^{\prime},
$$

a estatística escore eficiente é dada por

$$
T_{\widetilde{X}}=\sum_{i=1}^{n} \widetilde{U}_{i \beta}\left(\tilde{\theta}_{0}\right)
$$

onde $\tilde{\theta}_{0}=\left(\widetilde{\alpha}_{0}, 0^{\prime}\right)^{\prime}$, com $\widetilde{\alpha}_{0}$ satisfazendo

$$
\sum_{i=1}^{n} \tilde{U}_{i \alpha}\left(\tilde{\theta}_{0}\right)=0
$$

No lema a seguir mostramos que $\widetilde{\alpha}_{0}=\widehat{\alpha}_{0}$ e que $T_{\widetilde{X}}$ pode ser facilmente obtida a partir da expressão para $T_{Z}$.

Lema 3.1 Sob as suposições (i) e (ii),

$$
T_{\widetilde{X}}=\sum_{i=1}^{n} q_{i}\left(\widehat{\alpha}_{0}\right) E_{x_{i}}\left[z_{i}\right]
$$

\section{Prova:}

$$
\begin{aligned}
\tilde{U}_{i \alpha}\left(\tilde{\theta}_{0}\right) & =\left[\frac{\partial}{\partial \alpha} \log f\left(y_{i} \mid x_{i}\right)\right]_{\theta=\tilde{\theta}_{0}}=\left[\frac{1}{f\left(y_{i} \mid x_{i}\right)} \frac{\partial}{\partial \alpha} f\left(y_{i} \mid x_{i}\right)\right]_{\theta=\tilde{\theta}_{0}} \\
& =\left[\frac{1}{f\left(y_{i} \mid z_{i}\right)} \frac{\partial}{\partial \alpha} \int f\left(y_{i} \mid z_{i}\right) f\left(z_{i} \mid x_{i}\right) d z_{i}\right]_{\theta=\tilde{\theta}_{0}} \\
& =\left[\frac{1}{f\left(y_{i} \mid z_{i}\right)} \int f\left(z_{i} \mid x_{i}\right) \frac{\partial}{\partial \alpha} f\left(y_{i} \mid z_{i}\right) d z_{i}\right]_{\theta=\tilde{\theta}_{0}} \\
& =\left[\frac{1}{f\left(y_{i} \mid z_{i}\right)} \int f\left(z_{i} \mid x_{i}\right)\left[\frac{\partial}{\partial \alpha} \log f\left(y_{i} \mid z_{i}\right)\right] f\left(y_{i} \mid z_{i}\right) d z_{i}\right]_{\theta=\tilde{\theta}_{0}} \\
& =\int\left[\frac{\partial}{\partial \alpha} \log f\left(y_{i} \mid z_{i}\right)\right]_{\theta=\tilde{\theta}_{0}} f\left(z_{i} \mid x_{i}\right) d z_{i} \\
& =\left[\frac{\partial}{\partial \alpha} \log f\left(y_{i} \mid z_{i}\right)\right]_{\theta=\tilde{\theta}_{0}} \\
& =q_{i}\left(\tilde{\alpha}_{0}\right) \\
& =U_{i \alpha}\left(\tilde{\theta}_{0}\right) .
\end{aligned}
$$


Assim, $\sum_{i=1}^{n} \tilde{U}_{i \alpha}\left(\tilde{\theta}_{0}\right)=0$ se e somente se $\sum_{i=1}^{n} U_{i \alpha}\left(\tilde{\theta}_{0}\right)=0$. Segue de (3.25) que $\tilde{\theta}_{0}=\hat{\theta}_{0}$ ou seja, $\widetilde{\alpha}_{0}=\widehat{\alpha}_{0}$.

De forma análoga pode ser mostrado que

$$
\begin{aligned}
\tilde{U}_{i \beta}\left(\tilde{\theta}_{0}\right) & =\tilde{U}_{i \beta}\left(\hat{\theta}_{0}\right)=\int\left[\frac{\partial}{\partial \beta} \log f\left(y_{i} \mid z_{i}\right)\right]_{\theta=\hat{\theta}_{0}} f\left(z_{i} \mid x_{i}\right) d z_{i} \\
& =\int q_{i}\left(\hat{\alpha}_{0}\right) z_{i} f\left(z_{i} \mid x_{i}\right) d z_{i} \\
& =q_{i}\left(\hat{\alpha}_{0}\right) E_{x_{i}}\left[z_{i}\right],
\end{aligned}
$$

o que prova o lema.

Portanto, temos que $T_{\widetilde{X}}$ pode ser obtida substituindo $E_{x_{i}}\left[z_{i}\right]$ no lugar de $z_{i}$ na expressão para $T_{Z}$. Note que $T_{\widetilde{X}}$ requer o conhecimento de $E_{x_{i}}\left[z_{i}\right]$. No que segue assumiremos $E_{x_{i}}\left[z_{i}\right]$ conhecida. Se $E_{x_{i}}\left[z_{i}\right]$ depende de um parâmetro de dimensão finita $\tau$, de modo que $E_{x_{i}}\left[z_{i}\right]=g\left(\tau ; x_{i}\right)$, é fácil ver que, se $\widehat{\tau}$ é um estimador de $\tau$ tal que $\widehat{\tau}-\tau=o_{p}\left(n^{-1 / 2}\right)$, substituindo $\widehat{E}_{x_{i}}\left[z_{i}\right]=g\left(\widehat{\tau} ; x_{i}\right)$ no lugar de $E_{x_{i}}\left[z_{i}\right]$ em $(3.26)$, obtemos um teste assintoticamente equivalente.

A estatística escore "naive" é baseada em $T_{X}$ e obtida substituindo $x_{i}$ no lugar de $z_{i}$ na expressão para $T_{Z}$, isto é,

$$
T_{X}=\sum_{i=1}^{n} q_{i}\left(\widehat{\alpha}_{0}\right) x_{i}
$$

Mostraremos a seguir que as estatísticas $n^{-1 / 2} T_{Z}, n^{-1 / 2} T_{X}$ e $n^{-1 / 2} T_{\widetilde{X}}$ têm distribuição assintótica normal sob $H_{0}: \beta=0$ e sob uma seqüência de alternativas $\beta^{(n)}$ tais que $n^{1 / 2} \beta^{(n)} \rightarrow \Delta$, onde $\|\Delta\|>0$, com matrizes de covariância que denotaremos por $C_{Z}, C_{X}$ e $C_{\widetilde{X}}$, respectivamente. Se estas matrizes são consistentemente estimadas por $\widehat{C}_{Z}, \widehat{C}_{X}$ e $C_{\widetilde{X}}$, então as estatísticas definidas por

$$
\begin{aligned}
Q_{Z} & =n^{-1} T_{Z}^{\prime} \widehat{C}_{Z}^{-1} T_{Z}, \\
Q_{X} & =n^{-1} T_{X}^{\prime} \widehat{C}_{X}^{-1} T_{X}, \\
Q_{\widetilde{X}} & =n^{-1} T_{\widetilde{X}}^{\prime} \widehat{C}_{\widetilde{X}}^{-1} T_{\widetilde{X}},
\end{aligned}
$$


terão distribuição assintótica qui-quadrado com $s$ graus de liberdade sob $H_{0}$. Os parâmetros de não centralidade de $Q_{Z}, Q_{X}$ e $Q_{\widetilde{X}}$ sob a seqüência de alternativas poderão ser usados no cálculo das eficiências relativas assintóticas.

Proposição 3.1 Dadas as suposições (i), (ii) e (iv),

(a) sob $H_{0}$

$$
n^{-1 / 2} T_{Z} \stackrel{\mathcal{D}}{\longrightarrow} N\left(0, C_{Z}\right), n^{-1 / 2} T_{X} \stackrel{\mathcal{D}}{\longrightarrow} N\left(0, C_{X}\right), n^{-1 / 2} T_{\widetilde{X}} \stackrel{\mathcal{D}}{\longrightarrow} N\left(0, C_{\tilde{X}}\right),
$$

(b) sob uma seqüência de alternativas $\beta^{(n)}: n^{1 / 2} \beta^{(n)} \rightarrow \Delta,\|\Delta\|>0$,

$$
\begin{aligned}
& n^{-1 / 2} T_{Z} \stackrel{\mathcal{D}}{\longrightarrow} N\left(m_{Z}, C_{Z}\right), n^{-1 / 2} T_{X} \stackrel{\mathcal{D}}{\longrightarrow} N\left(m_{X}, C_{X}\right), n^{-1 / 2} T_{\widetilde{X}} \stackrel{\mathcal{D}}{\longrightarrow} N\left(m_{\widetilde{X}}, C_{\widetilde{X}}\right), \\
& \text { onde } C_{Z}=c \Sigma_{z}, C_{X}=c\left(\Sigma_{z}+\Sigma_{u}\right), C_{\widetilde{X}}=c \operatorname{Var}\left[E_{x}[z]\right], m_{Z}=\Sigma_{z} \Delta, m_{X}=\Sigma_{z} \Delta, \\
& m_{\tilde{X}}=\operatorname{Var}\left[E_{x}[z]\right] \Delta, \operatorname{com} c=\frac{1}{\phi}\{\dot{\mu}(\alpha)\}^{2} V^{-1}\{\mu(\alpha)] .
\end{aligned}
$$

Prova: (a) Seja $\theta_{0}=(\alpha, 0)^{\prime}$, supondo $\alpha$ conhecido definimos

$$
t_{Z}=\sum_{i=1}^{n} q_{i}(\alpha) z_{i}, t_{X}=\sum_{i=1}^{n} q_{i}(\alpha) x_{i}, t_{\widetilde{X}}=\sum_{i=1}^{n} q_{i}(\alpha) E_{x_{i}}\left[z_{i}\right] \quad \text { e } \quad t=\sum_{i=1}^{n} q_{i}(\alpha) .
$$

Por expansões em séries de Taylor, podemos expressar $T_{Z}, T_{X}$ e $T_{\widetilde{X}}$ como combinações lineares de $t_{Z}, t_{X}, t_{\widetilde{X}}$ e $t$, de modo que as distribuições assintóticas das estatísticas $n^{-1 / 2} T_{Z}$, $n^{-1 / 2} T_{X}$ e $n^{-1 / 2} T_{\widetilde{X}}$ podem ser obtidas a partir da distribuição assintótica de $t_{Z}, t_{X}, t_{\widetilde{X}}$ e $t$.

Da definição de $q_{i}$ em (3.23), é fácil ver que $E\left[q_{i}(\alpha) \mid z_{i}\right]=0$, de modo que $E\left[q_{i}(\alpha) z_{i}\right]=$ $E\left[q_{i}(\alpha) x_{i}\right]=E\left[q_{i}(\alpha) E_{x_{i}}\left[z_{i}\right]\right]=E\left[q_{i}(\alpha)\right]=0$. Por outro lado,

$$
\operatorname{Var}\left[q_{i}(\alpha) z_{i} \mid z_{i}\right]=\operatorname{Var}\left[q_{i}(\alpha) \mid z_{i}\right] z_{i} z_{i}^{\prime}=\frac{1}{\phi}\{\dot{\mu}(\alpha)\}^{2} V^{-1}\{\mu(\alpha)\} z_{i} z_{i}^{\prime}
$$


de modo que $\operatorname{Var}\left[q_{i}(\alpha) z_{i}\right]=c E\left(z_{i} z_{i}^{\prime}\right)$, onde $c=\frac{1}{\phi}\{\dot{\mu}(\alpha)\}^{2} V^{-1}\{\mu(\alpha)\}$. Analogamente encontram-se as variâncias de $q_{i}(\alpha) x_{i}, q_{i}(\alpha) E_{x_{i}}\left[z_{i}\right]$ e $q_{i}(\alpha)$, e as covariâncias correspondentes. Assim, a matriz de covariâncias de $q_{i}(\alpha)\left(z_{i}^{\prime}, x_{i}^{\prime}, E_{x_{i}}\left[z_{i}\right]^{\prime}, 1\right)^{\prime}$ pode ser escrita como

$$
C=\left(\begin{array}{llll}
C_{1} & C_{2} & C_{3} & C_{4} \\
C_{2}^{\prime} & C_{5} & C_{2}^{\prime} & C_{6} \\
C_{3}^{\prime} & C_{2} & C_{3} & C_{4} \\
C_{4}^{\prime} & C_{6}^{\prime} & C_{4}^{\prime} & C_{7}
\end{array}\right)
$$

onde $C_{1}=C_{2}=c\left(\Sigma_{z}+\mu \mu^{\prime}\right), C_{3}=c\left(\operatorname{Var}\left[E_{x}[z]\right]+\mu \mu^{\prime}\right), C_{4}=C_{6}=c \mu, C_{5}=c\left(\Sigma_{z}+\Sigma_{u}+\right.$ $\left.\mu \mu^{\prime}\right), C_{7}=c$.

Portanto, quando $\beta=0$, pelo Teorema Central do Limite Multivariado,

$$
n^{-1 / 2}\left(t_{Z}^{\prime}, t_{X}^{\prime}, t_{\widetilde{X}}^{\prime}, t\right)^{\prime} \stackrel{\mathcal{D}}{\longrightarrow} N=\left(N_{1}^{\prime}, N_{2}^{\prime}, N_{3}^{\prime}, N_{4}\right)^{\prime}
$$

onde $N$ é normal multivariada com vetor de médias 0 e matriz de covariâncias $C$, sendo $N_{1}, N_{2}, N_{3}$ e $N_{4}$ normais multivariadas com média 0 e matrizes de covariâncias $C_{1}, C_{5}$, $C_{3}$ e $C_{7}$, respectivamente. Expandindo em séries de Taylor, temos

$$
n^{-1 / 2} \sum_{i=1}^{n} U_{i}\left(\hat{\theta}_{0}\right)=n^{-1 / 2} \sum_{i=1}^{n} U_{i}\left(\theta_{0}\right)+n^{-1 / 2} \sum_{i=1}^{n} \frac{\partial}{\partial \theta} U_{i}\left(\theta^{*}\right)\left(\hat{\theta}_{0}-\theta_{0}\right)
$$

onde $\theta^{*}=\left(\alpha^{*}, 0\right), \operatorname{com}\left|\alpha^{*}-\alpha\right|<\left|\widehat{\alpha}_{0}-\alpha\right|$.

A expressão (3.33) pode ser reescrita como

$$
n^{-1 / 2} \sum_{i=1}^{n} U_{i \alpha}\left(\widehat{\theta}_{0}\right)=n^{-1 / 2} \sum_{i=1}^{n} U_{i \alpha}\left(\theta_{0}\right)+n^{-1 / 2} \sum_{i=1}^{n} \frac{\partial}{\partial \alpha} U_{i \alpha}\left(\theta^{*}\right)\left(\widehat{\alpha}_{0}-\alpha\right)
$$

e

$$
n^{-1 / 2} \sum_{i=1}^{n} U_{i \beta}\left(\hat{\theta}_{0}\right)=n^{-1 / 2} \sum_{i=1}^{n} U_{i \beta}\left(\theta_{0}\right)+n^{-1 / 2} \sum_{i=1}^{n} \frac{\partial}{\partial \beta} U_{i \alpha}\left(\theta^{*}\right)\left(\widehat{\alpha}_{0}-\alpha\right),
$$

de modo que, a partir de (3.25) e das definições de $t_{Z}, T_{Z}$ e $t$, temos que

$$
0=n^{-1 / 2} t+n^{-1 / 2} \sum_{i=1}^{n} \frac{\partial}{\partial \alpha} U_{i \alpha}\left(\theta^{*}\right)\left(\widehat{\alpha}_{0}-\alpha\right)
$$

e

$$
n^{-1 / 2} T_{Z}=n^{-1 / 2} t_{Z}+n^{-1 / 2} \sum_{i=1}^{n} \frac{\partial}{\partial \beta} U_{i \alpha}\left(\theta^{*}\right)\left(\widehat{\alpha}_{0}-\alpha\right)
$$


Como $\widehat{\alpha}_{0}$ é consistente quando $\beta=0$, pela lei fraca dos grandes números, temos que

$$
\begin{array}{ll}
\frac{1}{n} \sum_{i=1}^{n} \frac{\partial}{\partial \alpha} U_{i \alpha}\left(\theta^{*}\right) \stackrel{\mathrm{p}}{\longrightarrow} E\left[\frac{\partial}{\partial \alpha} U_{i \alpha}\left(\theta_{0}\right)\right] & =-C_{7}, \\
\frac{1}{n} \sum_{i=1}^{n} \frac{\partial}{\partial \beta} U_{i \alpha}\left(\theta^{*}\right) \stackrel{\mathrm{p}}{\longrightarrow} E\left[\frac{\partial}{\partial \beta} U_{i \alpha}\left(\theta_{0}\right)\right] & =-C_{4} .
\end{array}
$$

Portanto, usando (3.35) e (3.36), a expressão (3.34) pode ser reescrita como

$$
0=n^{-1 / 2} t-C_{7} \sqrt{n}\left(\widehat{\alpha}_{0}-\alpha\right)+o_{p}(1)
$$

$\mathrm{e}$

$$
n^{-1 / 2} T_{Z}=n^{-1 / 2} t_{Z}-C_{4} \sqrt{n}\left(\hat{\alpha}_{0}-\alpha\right)+o_{p}(1) .
$$

Substituindo (3.37) em (3.38), temos que

$$
n^{-1 / 2} T_{Z}=n^{-1 / 2}\left(t_{Z}-C_{4} C_{7}^{-1} t\right)+o_{p}(1)
$$

Analogamente, prova-se que

$$
\begin{aligned}
& n^{-1 / 2} T_{X}=n^{-1 / 2}\left(t_{X}-C_{6} C_{7}^{-1} t\right)+o_{p}(1) \\
& n^{-1 / 2} T_{\tilde{X}}=n^{-1 / 2}\left(t_{\widetilde{X}}-C_{4} C_{7}^{-1} t\right)+o_{p}(1) .
\end{aligned}
$$

As expressões (3.39), (3.40) e (3.41), junto com (3.32) e o Teorema de Cramer-Wold, implicam que, sob $H_{0}$,

$$
\begin{array}{ll}
n^{-1 / 2} T_{Z} & \stackrel{\mathcal{D}}{\longrightarrow} N\left(0, C_{Z}\right), \\
n^{-1 / 2} T_{X} & \stackrel{\mathcal{D}}{\longrightarrow} N\left(0, C_{X}\right), \\
n^{-1 / 2} T_{\widetilde{X}} \stackrel{\mathcal{D}}{\longrightarrow} N\left(0, C_{\tilde{X}}\right),
\end{array}
$$

onde

$$
\begin{aligned}
& C_{Z}=C_{1}-C_{4} C_{7}^{-1} C_{4}^{\prime}=c \Sigma_{z}, \\
& C_{X}=C_{5}-C_{6} C_{7}^{-1} C_{6}^{\prime}=c\left(\Sigma_{z}+\Sigma_{u}\right), \\
& C_{\tilde{X}}=C_{3}-C_{4} C_{7}^{-1} C_{4}^{\prime}=c \operatorname{Var}\left[E_{x}[z]\right] .
\end{aligned}
$$


(b) Sob $\beta=\beta^{(n)}, \hat{\alpha}$ também é consistente, de modo que as representações (3.39), (3.40) e (3.41) continuam valendo.

Sejam $t_{Z}^{(n)}, t_{X}^{(n)}, t_{\widetilde{X}}^{(n)}$ e $t^{(n)}$ definidas como $t_{Z}, t_{X}, t_{\widetilde{X}}$ e $t$ com $q_{i}(\alpha)$ substituído por $q_{i}\left(\alpha+\beta^{(n)^{\prime}} z_{i}\right)$. Pode ser mostrado, aplicando novamente o Teorema Central do Limite Multivaridado, que

$$
n^{-1 / 2}\left(t_{Z}^{(n)^{\prime}}, t_{X}^{(n)^{\prime}}, t_{\widetilde{X}}^{(n)^{\prime}}, t^{(n)}\right)^{\prime} \stackrel{\mathcal{D}}{\longrightarrow} N
$$

com $N$ como em (3.32).

Novamente, expansões em séries de Taylor podem ser utilizadas para mostrar que

$$
\begin{aligned}
& n^{-1 / 2} \sum_{i=1}^{n} U_{i \alpha}\left(\theta_{0}\right)=n^{-1 / 2} \sum_{i=1}^{n} U_{i \alpha}\left(\theta^{(n)}\right)-\frac{1}{n} \sum_{i=1}^{n} \frac{\partial}{\partial \beta^{\prime}} U_{i \alpha}\left(\theta^{*}\right) n^{1 / 2} \beta^{(n)}, \\
& n^{-1 / 2} \sum_{i=1}^{n} U_{i \beta}\left(\theta_{0}\right)=n^{-1 / 2} \sum_{i=1}^{n} U_{i \beta}\left(\theta^{(n)}\right)-\frac{1}{n} \sum_{i=1}^{n} \frac{\partial}{\partial \beta^{\prime}} U_{i \beta}\left(\theta^{*}\right) n^{1 / 2} \beta^{(n)},
\end{aligned}
$$

onde $\theta^{(n)}=\left(\alpha, \beta^{(n)^{\prime}}\right)^{\prime}$ e $\theta^{*}=\left(\alpha, \beta^{*^{\prime}}\right)^{\prime}$ com $\left\|\beta^{*}\right\|<\left\|\beta^{(n)}\right\|$. Pela lei fraca dos grandes números,

$$
\frac{1}{n} \sum_{i=1}^{n} \frac{\partial}{\partial \beta^{\prime}} U_{i \alpha}\left(\theta^{*}\right) \stackrel{\mathrm{p}}{\longrightarrow}-C_{4}^{\prime}
$$

e

$$
\frac{1}{n} \sum_{i=1}^{n} \frac{\partial}{\partial \beta^{\prime}} U_{i \beta}\left(\theta^{*}\right) \stackrel{\mathrm{p}}{\longrightarrow}-C_{1} .
$$

de modo que as expressões (3.43) podem ser reescritas como

$$
\begin{aligned}
n^{-1 / 2} t & =n^{-1 / 2} t^{(n)}+C_{4}^{\prime} n^{1 / 2} \beta^{(n)}+o_{p}(1), \\
n^{-1 / 2} t_{Z} & =n^{-1 / 2} t_{Z}^{(n)}+C_{1} n^{1 / 2} \beta^{(n)}+o_{p}(1) .
\end{aligned}
$$

Substituindo (3.44) e (3.45) em (3.39), temos que

$$
n^{-1 / 2} T_{Z}=n^{-1 / 2}\left(t_{Z}^{(n)}-C_{4} C_{7}^{-1} t^{(n)}\right)+\left(C_{1}-C_{4} C_{7}^{-1} C_{4}^{\prime}\right) n^{1 / 2} \beta^{(n)}+o_{p}(1) .
$$

Como $n^{1 / 2} \beta^{(n)} \rightarrow \Delta$, de (3.46), (3.42) e o Teorema de Cramer-Wold, segue que, sob uma seqüência de alternativas $\beta^{(n)}$,

$$
n^{-1 / 2} T_{Z} \stackrel{\mathcal{D}}{\longrightarrow} N\left(m_{Z}, C_{Z}\right)
$$


onde $m_{Z}=\left(C_{1}-C_{4} C_{7}^{-1} C_{4}^{\prime}\right) \Delta=c \Sigma_{z} \Delta$.

Analogamente, prova-se que sob a seqüencia de alternativas $\beta^{(n)}$,

$$
\begin{array}{ll}
n^{-1 / 2} T_{X} & \stackrel{\mathcal{D}}{\longrightarrow} N\left(m_{X}, C_{X}\right), \\
n^{-1 / 2} T_{\tilde{X}} \stackrel{\mathcal{D}}{\longrightarrow} N\left(m_{\tilde{X}}, C_{\tilde{X}}\right),
\end{array}
$$

onde

$$
m_{X}=\left(C_{2}-C_{6} C_{7}^{-1} C_{4}^{\prime}\right) \Delta=c \Sigma_{z} \Delta
$$

$\mathrm{e}$

$$
m_{\tilde{X}}=\left(C_{3}-C_{4} C_{7}^{-1} C_{4}^{\prime}\right) \Delta=c \operatorname{Var}\left[E_{x}[z]\right] \Delta .
$$

Denotando por $\widehat{C}_{Z}, \widehat{C}_{X}$ e $\widehat{C}_{\widetilde{X}}$ os estimadores de $C_{Z}, C_{X}$ e $C_{\widetilde{X}}$, sob a seqüência de alternativas temos que

$$
\begin{aligned}
Q_{Z} & =n^{-1} T_{Z}^{\prime} \widehat{C}_{Z}^{-1} T_{Z} \stackrel{\mathcal{D}}{\longrightarrow} \chi^{2}\left(M_{Z}\right), \\
Q_{X} & =n^{-1} T_{X}^{\prime} \widehat{C}_{X}^{-1} T_{X} \stackrel{\mathcal{D}}{\longrightarrow} \chi^{2}\left(M_{X}\right), \\
Q_{\tilde{X}} & =n^{-1} T_{\widetilde{X}}^{\prime} \widehat{C}_{\widetilde{X}}^{-1} T_{\tilde{X}} \stackrel{\mathcal{D}}{\longrightarrow} \chi^{2}\left(M_{\tilde{X}}\right),
\end{aligned}
$$

onde os parâmetros de não centralidade $M_{Z}, M_{X}$ e $M_{\tilde{X}}$ estão dados por

$$
\begin{aligned}
M_{Z} & =\Delta^{\prime}\left(C_{1}-C_{4} C_{7}^{-1} C_{4}^{\prime}\right) \Delta=c \Delta^{\prime} \Sigma_{z} \Delta, \\
M_{X} & =\Delta^{\prime}\left(C_{2}^{\prime}-C_{4} C_{7}^{-1} C_{6}^{\prime}\right)\left(C_{5}-C_{6} C_{7}^{-1} C_{6}^{\prime}\right)^{-1}\left(C_{2}-C_{6} C_{7}^{-1} C_{4}^{\prime}\right) \Delta \\
& =c \Delta^{\prime} \Sigma_{z}\left(\Sigma_{z}+\Sigma_{u}\right)^{-1} \Sigma_{z} \Delta, \\
M_{\widetilde{X}} & =\Delta^{\prime}\left(C_{3}-C_{4} C_{7}^{-1} C_{4}^{\prime}\right) \Delta=c \Delta^{\prime} \operatorname{Var}\left[E_{x}[z]\right] \Delta .
\end{aligned}
$$

Proposição 3.2 Dadas as suposições (i), (ii) e (iv),

$$
\operatorname{ARE}\left(T_{\tilde{X}}: T_{Z}\right) \leq 1, \operatorname{ARE}\left(T_{X}: T_{Z}\right) \leq 1 \quad \text { e } \operatorname{ARE}\left(T_{X}: T_{\tilde{X}}\right) \leq 1
$$


Prova: As eficiências relativas assintóticas são dadas por $\operatorname{ARE}\left(T_{\widetilde{X}}: T_{Z}\right)=\frac{M_{\tilde{x}}}{M_{Z}}, \operatorname{ARE}\left(T_{X}\right.$ : $\left.T_{Z}\right)=\frac{M_{X}}{M_{Z}}$ e $\operatorname{ARE}\left(T_{X}: T_{\tilde{X}}\right)=\frac{M_{X}}{M_{\tilde{X}}}$ (veja por exemplo, Cox e Hinkley, 1973).

De $(3.50)$ e (3.52), temos que

$$
M_{Z}-M_{\widetilde{X}}=c \Delta^{\prime}\left(\Sigma_{z}-\operatorname{Var}\left[E_{x}[z]\right]\right) \Delta=c \Delta^{\prime} E\left[\operatorname{Var}_{x}[z]\right] \Delta \geq 0
$$

de modo que $\operatorname{ARE}\left(T_{\widetilde{X}}: T_{Z}\right) \leq 1$

Em termos da distribuição normal multivariada $N$ definida em (3.32), os parâmetros de não centralidade $M_{Z}, M_{X}$ e $M_{\tilde{X}}$ podem ser escritos, usando as fórmulas das variâncias condicionais para vetores aleatórios normais (veja Anderson, 1984) como

$$
\begin{aligned}
M_{Z} & =\Delta^{\prime} \operatorname{Var}\left(N_{1} \mid N_{4}\right) \Delta, \\
M_{X} & \left.=\Delta^{\prime} \operatorname{Var}\left(N_{1} \mid N_{4}\right)-\operatorname{Var}\left(N_{1} \mid N_{4}, N_{2}\right)\right\} \Delta \\
& \left.=\Delta^{\prime} \operatorname{Var}\left(N_{3} \mid N_{4}\right)-\operatorname{Var}\left(N_{3} \mid N_{4}, N_{2}\right)\right\} \Delta, \\
M_{\tilde{X}} & =\Delta^{\prime} \operatorname{Var}\left(N_{3} \mid N_{4}\right) \Delta,
\end{aligned}
$$

de modo que (3.53) e (3.54) implicam $\frac{M_{X}}{M_{Z}} \leq 1$ e (3.55) e (3.56) implicam $\frac{M_{X}}{M_{\tilde{X}}} \leq 1$.

Corolário 3.1 Se $s=1$, então $\operatorname{ARE}\left(T_{X}: T_{Z}\right)=\operatorname{corr}(z, x)^{2}$ e $\operatorname{ARE}\left(T_{X}: T_{\tilde{X}}\right)=$ $\operatorname{corr}\left(E_{x}[z], x\right)^{2}$.

Prova: Se $s=1$, temos que $z \sim\left(\mu, \sigma_{z}^{2}\right)$ e $u \sim\left(0, \sigma_{u}^{2}\right)$, então

$$
M_{Z}=c \Delta^{2} \sigma_{z}^{2}, M_{X}=c \Delta^{2} \frac{\sigma_{z}^{4}}{\sigma_{z}^{2}+\sigma_{u}^{2}} \quad \text { e } \quad M_{\widetilde{X}}=c \Delta^{2} \operatorname{Var}\left[E_{x}[z]\right]
$$

de modo que

$$
\operatorname{ARE}\left(T_{X}: T_{Z}\right)=\frac{\sigma_{z}^{2}}{\sigma_{z}^{2}+\sigma_{u}^{2}}=\operatorname{corr}(z, x)^{2}
$$

e

$$
\operatorname{ARE}\left(T_{X}: T_{\widetilde{X}}\right)=\frac{\sigma_{z}^{4}}{\left(\sigma_{z}^{2}+\sigma_{u}^{2}\right) \operatorname{Var}\left[E_{x}[z]\right]}=\operatorname{corr}\left(E_{x}[z], x\right)^{2},
$$

desde que $\operatorname{Cov}(z, x)=\operatorname{Cov}\left(E_{x}[z], x\right)=\sigma_{z}^{2}$. 
Para a construção das estatísticas $Q_{Z}, Q_{X}$ e $Q_{\widetilde{X}}$, precisamos estimar consistentemente as matrizes $C_{Z}, C_{X}$ e $C_{\widetilde{X}}$, onde $C_{Z}=c \Sigma_{z}, C_{X}=c\left(\Sigma_{z}+\Sigma_{u}\right)$ e $C_{\tilde{X}}=c \operatorname{Var}\left[E_{x}(z)\right]$, sendo $c=\frac{1}{\phi}\{\dot{\mu}(\alpha)\}^{2} V^{-1}\{\mu(\alpha)\}$.

Dado que $z_{i} \stackrel{\text { iid }}{\sim}\left(\mu, \Sigma_{z}\right), i=1, \ldots, n$, independentes dos erros de medição $u_{i}$, onde $u_{i} \stackrel{\text { iid }}{\sim}\left(0, \Sigma_{u}\right)$, medições replicadas das $z_{i}$ 's podem ser usadas para obter estimadores de $\Sigma_{u}$ e $\Sigma_{z}$, e conseqüentemente estimadores de $C_{Z}$ e $C_{X}$.

Suponha que dispomos de $k_{i}$ medições replicadas de $x_{i}$, ou seja,

$$
x_{i j}=z_{i}+u_{i j}, \quad j=1, \ldots, k_{i}, i=1, \ldots, n .
$$

Assim, os parâmetros $\Sigma_{u}$ e $\Sigma_{z}$ podem ser estimados usando as fórmulas da análise de componentes variância,

$$
\hat{\Sigma}_{u}=\frac{\sum_{i=1}^{n} \sum_{j=1}^{k_{i}}\left(x_{i j}-\bar{x}_{i .}\right)\left(x_{i j}-\bar{x}_{i .}\right)^{\prime}}{\sum_{i=1}^{n}\left(k_{i}-1\right)}
$$

e

$$
\widehat{\Sigma}_{z}=\frac{\left\{\sum_{i=1}^{n} k_{i}\left(\bar{x}_{i}-\bar{x}\right)\left(\bar{x}_{i}-\bar{x}\right)^{\prime}\right\}-(n-1) \hat{\Sigma}_{u}}{\sum_{i=1}^{n} k_{i}-\sum_{i=1}^{n} k_{i}^{2} / \sum_{i=1}^{n} k_{i}}
$$

onde

$$
\bar{x}_{i} .=\frac{1}{k_{i}} \sum_{j=1}^{k_{i}} x_{i j} \quad \text { e } \quad \bar{x}=\frac{\sum_{i=1}^{n} k_{i} x_{i}}{\sum_{i=1}^{n} k_{i}}=\widehat{\mu} .
$$

Estimadores consistentes de $C_{Z}$ e $C_{X}$ são dados por $\widehat{C}_{Z}=\hat{c} \widehat{\Sigma}_{z}$ e $\widehat{C}_{X}=\widehat{c}\left(\widehat{\Sigma}_{z}+\widehat{\Sigma}_{u}\right)$, respectivamente, onde $\hat{c}=\frac{1}{\phi}\left\{\dot{\mu}\left(\hat{\alpha}_{0}\right)\right\}^{2} V^{-1}\left\{\mu\left(\hat{\alpha}_{0}\right)\right\}$, com $\hat{\alpha}_{0}$ solução de (3.25).

Para a construção da estatística $Q_{\tilde{X}}$ precisamos do conhecimento de $E_{x}[z]$. Considere como exemplo o caso em que $z \sim N\left(\mu, \Sigma_{z}\right)$ e $u \sim N\left(0, \Sigma_{u}\right)$, de modo que $E_{x}[z]=$ $\mu+\Sigma_{z}\left(\Sigma_{z}+\Sigma_{u}\right)^{-1}(x-\mu)=\mu\left[I_{s}-\Sigma_{z}\left(\Sigma_{z}+\Sigma_{u}\right)^{-1}\right]+\Sigma_{z}\left(\Sigma_{z}+\Sigma_{u}\right)^{-1} x$, e portanto $\operatorname{Var}\left[E_{x}[z]\right]=$ $\Sigma_{z}\left(\Sigma_{z}+\Sigma_{u}\right)^{-1} \Sigma_{z}$. Então, um estimador de $C_{\widetilde{X}}$ é dado por $\widehat{C}_{\tilde{X}}=\widehat{c} \widehat{\Sigma}_{z}\left(\widehat{\Sigma}_{z}+\widehat{\Sigma}_{u}\right)^{-1} \widehat{\Sigma}_{z}$. Assim,

$$
\begin{aligned}
T_{\widetilde{X}} & =\sum_{i=1}^{n} q_{i}\left(\widehat{\alpha}_{0}\right) \widehat{E}_{x_{i}}\left[z_{i}\right] \\
& =\sum_{i=1}^{n} q_{i}\left(\widehat{\alpha}_{0}\right)\left\{\hat{\mu}\left[I_{s}-\widehat{\Sigma}_{z}\left(\widehat{\Sigma}_{z}+\widehat{\Sigma}_{u}\right)^{-1}\right]+\widehat{\Sigma}_{z}\left(\widehat{\Sigma}_{z}+\widehat{\Sigma}_{u}\right)^{-1} x_{i}\right\}
\end{aligned}
$$




$$
\begin{aligned}
& =\hat{\Sigma}_{z}\left(\hat{\Sigma}_{z}+\hat{\Sigma}_{u}\right)^{-1} \sum_{i=1}^{n} q_{i}\left(\hat{\alpha}_{0}\right) x_{i} \\
& =\hat{\Sigma}_{z}\left(\hat{\Sigma}_{z}+\hat{\Sigma}_{u}\right)^{-1} T_{X}
\end{aligned}
$$

desde que $\sum_{i=1}^{n} q_{i}\left(\hat{\alpha}_{0}\right)=0$ por (3.25). Portanto,

$$
Q_{\widetilde{X}}=n^{-1} T_{\widetilde{X}}^{\prime} \widehat{C}_{\widetilde{X}}^{-1} T_{\widetilde{X}}=n^{-1} T_{X}^{\prime} \widetilde{c}^{-1}\left(\widehat{\Sigma}_{z}+\widehat{\Sigma}_{u}\right)^{-1} T_{X}=Q_{X}
$$

isto é, quando $z$ e $u$ são normalmente distribuídas as estatísticas $Q_{\widetilde{X}}$ e $Q_{X}$ coincidem, $\forall n$. $\mathrm{Na}$ verdade, este resultado pode ser mostrado sempre que $E_{x}[z]$ seja função linear de $x$.

Proposição 3.3 Se $E_{x}[z]=a+B x$, onde a é um vetor $s \times 1$ e $B$ uma matriz $s \times s$, definida positiva, então $Q_{\tilde{X}}=Q_{X}$.

Prova: $\operatorname{Var}\left[E_{x}[z]\right]=B \operatorname{Var}(x) B^{\prime}=B\left(\Sigma_{z}+\Sigma_{u}\right) B^{\prime}$, de modo que $\widehat{C}_{\widetilde{X}}=\hat{c} \widehat{B}\left(\widehat{\Sigma}_{z}+\widehat{\Sigma}_{u}\right) \widehat{B}^{\prime}$, onde $\widehat{B}$ é um estimador consistente da matriz $B$. Assim,

$$
\begin{aligned}
T_{\widetilde{X}} & =\sum_{i=1}^{n} q_{i}\left(\widehat{\alpha}_{0}\right) \widehat{E}_{x_{i}}\left[z_{i}\right]=\sum_{i=1}^{n} q_{i}\left(\widehat{\alpha}_{0}\right)\left(\widehat{a}+\widehat{B} x_{i}\right) \\
& =\hat{a} \sum_{i=1}^{n} q_{i}\left(\widehat{\alpha}_{0}\right)+\widehat{B} \sum_{i=1}^{n} q_{i}\left(\widehat{\alpha}_{0}\right) x_{i} \\
& =\hat{B} T_{X} .
\end{aligned}
$$

Portanto,

$$
\begin{aligned}
Q_{\tilde{X}} & =n^{-1} T_{\widetilde{X}}^{\prime} \widehat{C}_{\widetilde{X}}^{-1} T_{\widetilde{X}}=n^{-1} T_{X}^{\prime} \widehat{B}^{\prime} \widehat{c}^{-1}\left(\hat{B}^{\prime}\right)^{-1}\left(\widehat{\Sigma}_{z}+\widehat{\Sigma}_{u}\right)^{-1}(\widehat{B})^{-1} \hat{B} T_{X} \\
& =n^{-1} T_{X}^{\prime} \widehat{c}^{-1}\left(\widehat{\Sigma}_{z}+\widehat{\Sigma}_{u}\right)^{-1} T_{X}=Q_{X}
\end{aligned}
$$

Sob uma estrutura de erros de medição mais geral do que a considerada aqui, Tosteson e Tsiatis (1988) notam que, se $E_{x}[z]$ for função linear de $x$, então, $M_{X} / M_{\widetilde{X}}=1$. Assim, o teste "naive" $Q_{X}$ e o teste escore eficiente $Q_{\widetilde{X}}$ são assintoticamente equivalentes (Lindley, 1947, dá condições sob as quais $E_{x}[z]$ é linear). Na Proposição 3.3 mostramos que sob uma estrutura aditiva de erros de medição, um resultado mais forte pode ser obtido, isto é, $Q_{X}=Q_{\widetilde{X}}, \forall n$. 


\subsubsection{Teste escore corrigido}

A estatística escore corrigido $Q_{c}$, definida em (3.6) é dada por

$$
Q_{c}=n^{-1} T_{c}^{\prime} \hat{C}_{c}^{-1} T_{c}
$$

onde

$$
T_{c}=U_{\beta}^{*}\left(\widehat{\theta}_{c 0}\right)=\sum_{i=1}^{n} U_{i \beta}^{*}\left(\widehat{\theta}_{c 0}\right),
$$

$\operatorname{com} \hat{\theta}_{c 0}=\left(\widehat{\alpha}_{c 0}, 0^{\prime}\right)^{\prime}$ satisfazendo $\sum_{i=1}^{n} U_{i \alpha}^{*}\left(\hat{\theta}_{c 0}\right)=0$, sendo $U^{*}(\theta)$ o escore corrigido, e

$$
\widehat{C}_{c}=\widehat{\Lambda}_{\beta \beta . \alpha}\left(\hat{\theta}_{c 0}\right) \hat{\Omega}_{\beta \beta}^{-1}\left(\hat{\theta}_{c 0}\right) \widehat{\Lambda}_{\beta \beta . \alpha}\left(\hat{\theta}_{c 0}\right)
$$

(veja a Seção 3.2).

A seguir mostramos que sob as suposições (i) - (v) a função escore corrigida $U^{*}(\theta)$ pode ser facilmente obtida.

Lembrando a notação da Seção 3.3.1, o escore usual é dado por

$$
U(\theta)=\sum_{i=1}^{n} U_{i}(\theta)=\sum_{i=1}^{n}\left(U_{i \alpha}(\theta), U_{i \beta}(\theta)^{\prime}\right)^{\prime},
$$

com

$$
\begin{aligned}
& U_{i \alpha}=q_{i}\left(\alpha+\beta^{\prime} z_{i}\right), \\
& U_{i \beta}=q_{i}\left(\alpha+\beta^{\prime} z_{i}\right) z_{i},
\end{aligned}
$$

e sob a suposição (iii),

$$
q_{i}\left(\alpha+\beta^{\prime} z_{i}\right)=\frac{1}{\phi} \exp \left[(1-k)\left(\alpha+\beta^{\prime} z_{i}\right)\right]\left[y_{i}-\exp \left(\alpha+\beta^{\prime} z_{i}\right)\right]
$$

Dadas as suposições (iv) e (v) e as identidades (2.35) e (2.36) da Seção 2.6, para a função geradora de momentos de uma variável normal, é fácil mostrar que

$$
\begin{aligned}
U_{i \alpha}^{*}(\theta)= & \frac{1}{\phi}\left\{\exp \left[(1-k)\left(\alpha+\beta^{\prime} x_{i}\right)-\xi_{u}\right] y_{i}-\exp \left[(2-k)\left(\alpha+\beta^{\prime} x_{i}\right)-\gamma_{u}\right]\right\}, \\
U_{i \beta}^{*}(\theta)= & \frac{1}{\phi}\left\{\exp \left[(1-k)\left(\alpha+\beta^{\prime} x_{i}\right)-\xi_{u}\right]\left[x_{i}-(1-k) \Sigma_{u} \beta\right] y_{i}\right. \\
& \left.-\exp \left[(2-k)\left(\alpha+\beta^{\prime} x_{i}\right)-\gamma_{u}\right]\left[x_{i}-(2-k) \Sigma_{u} \beta\right]\right\},
\end{aligned}
$$


onde $\xi_{u}=\frac{(1-k)^{2}}{2} \beta^{\prime} \Sigma_{u} \beta$ e $\gamma_{u}=\frac{(2-k)^{2}}{2} \beta^{\prime} \Sigma_{u} \beta$.

$\mathrm{Na}$ proposição a seguir mostramos que a estatística $T_{c}$ definida em (3.57) e a estatística "naive" $T_{X}$ definida em (3.27) coincidem, $\forall n$.

Proposição 3.4 Sob as suposições (i) - (v), $T_{c}=T_{X}, \forall n$, onde $T_{c}$ e $T_{X}$ são definidas em (3.57) e (3.27), respectivamente. Conseqüentemente, $Q_{c}=Q_{X}, \forall n$.

Prova: $T_{c}=\sum_{i=1}^{n} U_{i \beta}^{*}\left(\widehat{\theta}_{c 0}\right)$, onde $\widehat{\theta}_{c 0}=\left(\widehat{\alpha}_{c 0}, 0^{\prime}\right)^{\prime}$ satisfaz $\sum_{i=1}^{n} U_{i \alpha}^{*}\left(\widehat{\theta}_{c 0}\right)=0$. Sob a suposição (iii),

$$
\begin{aligned}
\sum_{i=1}^{n} U_{i \alpha}^{*}\left(\widehat{\theta}_{c 0}\right) & =\sum_{i=1}^{n} \frac{1}{\phi}\left\{\exp \left[(1-k) \widehat{\alpha}_{c 0}\right] y_{i}-\exp \left[(2-k) \widehat{\alpha}_{c 0}\right]\right\} \\
& =\sum_{i=1}^{n} \frac{1}{\phi}\left\{\exp \left[(1-k) \widehat{\alpha}_{c 0}\right]\left[y_{i}-\exp \left(\widehat{\alpha}_{c 0}\right)\right]\right\}=0
\end{aligned}
$$

e por $(3.25)$,

$$
\sum_{i=1}^{n} U_{i \alpha}\left(\hat{\theta}_{0}\right)=\sum_{i=1}^{n} \frac{1}{\phi}\left\{\exp \left[(1-k) \widehat{\alpha}_{0}\right]\left[y_{i}-\exp \left(\hat{\alpha}_{0}\right)\right]=0,\right.
$$

de modo que $\widehat{\alpha}_{c 0}=\widehat{\alpha}_{0}$ e, portanto, $\widehat{\theta}_{c 0}=\widehat{\theta}_{0}$. Assim,

$$
\begin{aligned}
T_{c} & =\sum_{i=1}^{n} U_{i \beta}^{*}\left(\hat{\theta}_{c 0}\right)=\sum_{i=1}^{n} U_{i \beta}^{*}\left(\hat{\theta}_{0}\right)=\sum_{i=1}^{n} \frac{1}{\phi}\left\{\exp \left[(1-k) \widehat{\alpha}_{0}\right] y_{i}-\exp \left[(2-k) \widehat{\alpha}_{0}\right]\right\} x_{i} \\
& =\sum_{i=1}^{n} \frac{1}{\phi}\left\{\exp \left[(1-k) \hat{\alpha}_{0}\right]\left[y_{i}-\exp \left(\widehat{\alpha}_{0}\right)\right]\right\} x_{i} \\
& =T_{X} .
\end{aligned}
$$

Portanto, para a família de modelos definida pela suposição (iii), $T_{c}=T_{X}$. Conseqüentemente, a estatística do escore "naive" $Q_{X}$ e a estatística do escore corrigido $Q_{c}$ coincidem $\forall n$, quando testamos $H_{0}: \beta=0$, com $\alpha$ como parâmetro "nuisance". Juntando a isto a Proposição 3.3 , temos que sob estas suposições $Q_{X}=Q_{\widetilde{X}}=Q_{c}$.

Observação: É fácil ver que o resultado da Proposição 3.4 também vale para o modelo de regressão linear normal (note que este modelo pertence à família Tweedie, mas $E[y \mid z]=$ 
$\left.\mu\left(\alpha+\beta^{\prime} z\right)=\alpha+\beta^{\prime} z\right)$. Para este modelo

$$
U_{i \alpha}=\frac{1}{\phi}\left\{y_{i}-\left(\alpha+\beta^{\prime} z_{i}\right)\right\}
$$

$\mathrm{e}$

$$
U_{i \beta}=\frac{1}{\phi}\left\{y_{i}-\left(\alpha+\beta^{\prime} z_{i}\right)\right\} z_{i}
$$

de modo que

$$
U_{i \alpha}^{*}=\frac{1}{\phi}\left\{y_{i}-\left(\alpha+\beta^{\prime} x_{i}\right)\right\}
$$

$\mathrm{e}$

$$
U_{i \beta}^{*}=\frac{1}{\phi}\left\{y_{i}-\left(\alpha+\beta^{\prime} x_{i}\right)\right\} x_{i}+\frac{1}{\phi} \Sigma_{u} \beta .
$$

Assim, $\sum_{i=1}^{n} U_{i \alpha}^{*}\left(\hat{\theta}_{c 0}\right)=\sum_{i=1}^{n} U_{i \alpha}\left(\hat{\theta}_{c 0}\right)$ e portanto $\hat{\theta}_{c 0}=\hat{\theta}_{0}$ e $\sum_{i=1}^{n} U_{i \beta}^{*}\left(\hat{\theta}_{c 0}\right)=\sum_{i=1}^{n} U_{i \beta}^{*}\left(\hat{\theta}_{0}\right)=$ $\sum_{i=1}^{n} U_{i \beta}\left(\hat{\theta}_{0}\right)$.

\subsection{Comparação de testes de Wald}

Como na Seção 3.3.2, precisamos das suposições (i) - (v). Além disso, consideremos

$$
z_{i} \stackrel{\text { iid }}{\sim} N\left(\mu, \Sigma_{z}\right)
$$

Sob esta última condição o vício do estimador "naive" será obtido explicitamente.

Se o interesse é testar $H_{0}: \beta=0$, na presença do parâmetro "nuisance" $\alpha$, dado um estimador $\widehat{\beta}$ de $\beta$, tal que sob $H_{0}$

$$
n^{1 / 2} \widehat{\beta} \stackrel{\mathcal{D}}{\longrightarrow} N(0, D)
$$

e $\widehat{D}$ é estimador consistente de $D$, a estatística do teste de Wald é dada por

$$
W=n \widehat{\beta}^{\prime} \widehat{D}^{-1} \widehat{\beta}
$$

O objetivo nesta seção é comparar o comportamento assintótico do teste de Wald obtido quando as verdadeiras covariáveis $z_{i}$ são realmente observadas, com o teste de Wald "naive" e o teste de Wald corrigido $W_{c}$, definido em (3.5). Quando as $\left\{z_{i}\right\}_{i=1}^{n}$ são realmente 
observadas, denotaremos por $\hat{\theta}_{Z}=\left(\widehat{\alpha}_{Z}, \widehat{\beta}_{Z}^{\prime}\right)^{\prime}$ o EMV de $\theta$, ou seja, $\hat{\theta}_{Z}$ é solução da equação

$$
\sum_{i=1}^{n} U_{i}(\theta)=\sum_{i=1}^{n}\left(U_{i \alpha}(\theta), U_{i \beta}(\theta)^{\prime}\right)^{\prime}=\sum_{i=1}^{n} q_{i}\left(\alpha+\beta^{\prime} z_{i}\right)\left(1, z_{i}^{\prime}\right)^{\prime}=0,
$$

onde $q_{i}\left(\alpha+\beta^{\prime} z_{i}\right)$ é dada por (3.58).

A função escore "naive" é dada por

$$
\sum_{i=1}^{n} q_{i}\left(\alpha+\beta^{\prime} x_{i}\right)\left(1, x_{i}^{\prime}\right)^{\prime}
$$

e o EMV "naive", que denotaremos por $\widehat{\theta}_{X}=\left(\widehat{\alpha}_{X}, \widehat{\beta}_{X}^{\prime}\right)^{\prime}$ é solução da equação

$$
\sum_{i=1}^{n} q_{i}\left(\alpha+\beta^{\prime} x_{i}\right)\left(1, x_{i}^{\prime}\right)^{\prime}=0
$$

Em geral, $\hat{\theta}_{X}$ é assintoticamente viciado.

Um estimador consistente de $\theta$ pode ser obtido pelo método do escore corrigido. Denotaremos por $\widehat{\theta}_{c}=\left(\widehat{\alpha}_{c}, \widehat{\beta}_{c}^{\prime}\right)^{\prime}$, o estimador do escore corrigido, ou seja, $\hat{\theta}_{c}$ é solução da equação

$$
\sum_{i=1}^{n} U_{i}^{*}(\theta)=\sum_{i=1}^{n}\left(U_{i \alpha}^{*}(\theta), U_{i \beta}^{*}(\theta)^{\prime}\right)^{\prime}=0,
$$

onde $U_{i \alpha}^{*}(\theta)$ e $U_{i \beta}^{*}(\theta)$ são dadas por $(3.59)$ e $(3.60)$, respectivamente.

Denotaremos por $W_{Z}, W_{X}$ e $W_{c}$ as estatísticas de teste da forma (3.62) baseadas nos estimadores $\widehat{\beta}_{Z}, \widehat{\beta}_{X}$ e $\widehat{\beta}_{c}$, respectivamente. Note que $W_{c}$ corresponde à estatística do teste de Wald definida em (3.5).

No lema seguinte, que será útil no cálculo das eficiências relativas assintóticas, mostramos que para a família de modelos descritos pela suposição (iii), o vício assintótico do estimador "naive" pode ser obtido explicitamente.

Lema 3.2 Sob as suposições $(i)-(v)$ e (3.61), $\hat{\theta}_{X} \stackrel{\mathrm{p}}{\longrightarrow} \theta^{*}$, onde $\theta^{*}=\left(\alpha^{*}, \beta^{*^{\prime}}\right)^{\prime}$, com $\beta^{*}=R \beta, \alpha^{*}=\alpha+\mu^{\prime}\left(I_{s}-R\right) \beta+\frac{1}{2} \beta^{\prime} \Sigma_{z}\left(I_{s}-R\right) \beta$ e $R=\left(\Sigma_{z}+\Sigma_{u}\right)^{-1} \Sigma_{z}$, sendo $\theta=\left(\alpha, \beta^{\prime}\right)^{\prime}$ o verdadeiro valor do parâmetro. 
Prova: $\hat{\theta}_{X} \stackrel{\mathrm{p}}{\longrightarrow} \theta^{*}, \operatorname{com} \theta^{*}=\left(\alpha^{*}, \beta^{*^{\prime}}\right)^{\prime}$ satisfazendo

$$
E\left[q_{i}\left(\alpha^{*}+\beta^{*^{\prime}} x_{i}\right)\left(1, x_{i}^{\prime}\right)^{\prime}\right]=0, i=1, \ldots, n,
$$

onde a esperança é tomada sob o verdadeiro valor do parâmetro $\theta=\left(\alpha, \beta^{\prime}\right)^{\prime}$ (Stefanski, 1985). A equação (3.63) implica em

$$
\begin{aligned}
& E\left[q_{i}\left(\alpha^{*}+\beta^{*^{\prime}} x_{i}\right)\right]=0, \\
& E\left[q_{i}\left(\alpha^{*}+\beta^{*^{\prime}} x_{i}\right) x_{i}\right]=0,
\end{aligned}
$$

onde $q_{i}\left(\alpha^{*}+{\beta^{*^{\prime}}} x_{i}\right)=\frac{1}{\phi} \exp \left[(1-k)\left(\alpha^{*}+{\beta^{*^{\prime}}} x_{i}\right)\right]\left[y_{i}-\exp \left(\alpha^{*}+{\beta^{*^{\prime}}} x_{i}\right)\right]$. (3.64) e (3.65) podem ser escritas como

$$
\begin{gathered}
a-b=0 \\
a\left\{\mu+\Sigma_{z}\left[(1-k) \beta^{*}+\beta\right]+(1-k) \Sigma_{u} \beta^{*}\right\}-b\left\{\mu+(2-k) \Sigma_{z} \beta^{*}+(2-k) \Sigma_{u} \beta^{*}\right\}=0
\end{gathered}
$$

onde

$$
a=\exp \left\{(1-k) \alpha^{*}+\alpha+\mu^{\prime}\left[(1-k) \beta^{*}+\beta\right]+\xi_{u}^{*}+\psi_{z}^{*}\right\}
$$

e

$$
b=\exp \left\{(2-k)\left(\alpha^{*}+\beta^{*^{\prime}} \mu\right)+\gamma_{u}^{*}+\gamma_{z}^{*}\right\}
$$

com

$$
\begin{aligned}
& \xi_{u}^{*}=\frac{(1-k)^{2}}{2} \beta^{*^{\prime}} \Sigma_{u} \beta^{*}, \quad \gamma_{u}^{*}=\frac{(2-k)^{2}}{2} \beta^{*^{\prime}} \Sigma_{u} \beta^{*}, \\
& \psi_{z}^{*}=\frac{1}{2}\left[(1-k) \beta^{*}+\beta\right]^{\prime} \Sigma_{z}\left[(1-k) \beta^{*}+\beta\right], \quad \gamma_{z}^{*}=\frac{(2-k)^{2}}{2} \beta^{*^{\prime}} \Sigma_{z} \beta^{*} .
\end{aligned}
$$

Resolvendo (3.66) e (3.67), encontramos

$$
\beta^{*}=R \beta
$$

e

$$
\alpha^{*}=\alpha+\mu^{\prime}\left(I_{s}-R\right) \beta+\frac{1}{2} \beta^{\prime} \Sigma_{z}\left(I_{s}-R\right) \beta,
$$


onde $R=\left(\Sigma_{z}+\Sigma_{u}\right)^{-1} \Sigma_{z}$

Note que o vício do estimador "naive" do parâmetro $\beta$ comporta-se de acordo com a fórmula de "correção por atenuação" do modelo de regressão linear (Fuller, 1987).

Se o verdadeiro valor do parâmetro é $\theta=\theta_{0}=\left(\alpha, 0^{\prime}\right)^{\prime}$, então $\beta^{*}=0$ e $\alpha^{*}=\alpha$, de modo que sob $H_{0}, \theta^{*}=\theta_{0}$.

A seguir encontramos a distribuição assintótica das estatísticas $n^{1 / 2} \widehat{\beta}_{Z}, n^{1 / 2} \widehat{\beta}_{X}$ e $n^{1 / 2} \widehat{\beta}_{c}$ sob $H_{0}$ e sob uma seqüência de alternativas $\beta^{(n)}$ tais que $n^{1 / 2} \beta^{(n)} \rightarrow \Delta$, onde $\|\Delta\|>0$.

Proposição 3.5 Dadas as suposições (i) - (v) e (3.61),

(a) sob $H_{0}$,

$$
n^{1 / 2} \widehat{\beta}_{Z} \stackrel{\mathcal{D}}{\longrightarrow} N\left(0, D_{Z}\right), n^{1 / 2} \widehat{\beta}_{X} \stackrel{\mathcal{D}}{\longrightarrow} N\left(0, D_{X}\right) \quad \text { e } n^{1 / 2} \widehat{\beta}_{c} \stackrel{\mathcal{D}}{\longrightarrow} N\left(0, D_{c}\right),
$$

(b) sob uma seqüência de alternativas $\beta^{(n)}: n^{1 / 2} \beta^{(n)} \rightarrow \Delta,\|\Delta\|>0$,

$$
\begin{aligned}
& \qquad n^{1 / 2} \widehat{\beta}_{Z} \stackrel{\mathcal{D}}{\longrightarrow} N\left(\Delta, D_{Z}\right), n^{1 / 2} \widehat{\beta}_{X} \stackrel{\mathcal{D}}{\longrightarrow} N\left(R \Delta, D_{X}\right) \quad \text { e } n^{1 / 2} \widehat{\beta}_{c} \stackrel{\mathcal{D}}{\longrightarrow} N\left(\Delta, D_{c}\right), \\
& \text { onde } D_{Z}=d \Sigma_{z}^{-1}, D_{X}=d\left(\Sigma_{z}+\Sigma_{u}\right)^{-1} \text { e } D_{c}=d\left(I_{s}+\Sigma_{z}^{-1} \Sigma_{u}\right) \Sigma_{z}^{-1}, \text { com } R= \\
& \left(\Sigma_{z}+\Sigma_{u}\right)^{-1} \Sigma_{z} \text { ed } d=\phi \exp [(k-2) \alpha] .
\end{aligned}
$$

Prova: (a) Quando $z$ for realmente observada é sabido que $\widehat{\theta}_{Z}$ é tal que, sob $H_{0}, \widehat{\theta}_{Z} \stackrel{\mathrm{p}}{\longrightarrow} \theta_{0}$ e

$$
n^{1 / 2}\left(\hat{\theta}_{Z}-\theta_{0}\right) \stackrel{\mathcal{D}}{\longrightarrow} N\left(0, K^{-1}\left(\theta_{0}\right)\right)
$$

onde

$$
K\left(\theta_{0}\right)=E\left[-\dot{q}(\alpha)\left(1, z^{\prime}\right)^{\prime}\left(1, z^{\prime}\right)\right]=E[-\dot{q}(\alpha)]\left(\begin{array}{cc}
1 & \mu^{\prime} \\
\mu & \Sigma_{z}+\mu \mu^{\prime}
\end{array}\right)
$$

onde

$$
\dot{q}(\alpha)=\frac{1}{\phi} \exp [(1-k) \alpha](1-k)[y-\exp (\alpha)]-\frac{1}{\phi} \exp [(2-k) \alpha]
$$


Assim,

$$
K^{-1}\left(\theta_{0}\right)=\{E[-\dot{q}(\alpha)]\}^{-1}\left(\begin{array}{cc}
1+\mu^{\prime} \Sigma_{z}^{-1} \mu & -\mu^{\prime} \Sigma_{z}^{-1} \\
-\Sigma_{z}^{-1} \mu & \Sigma_{z}^{-1}
\end{array}\right) .
$$

É fácil ver que $E[-\dot{q}(\alpha)]=\frac{1}{\phi} \exp [(2-k) \alpha]$, de modo que sob $H_{0}$

$$
n^{1 / 2} \widehat{\beta}_{Z} \stackrel{\mathcal{D}}{\longrightarrow} N\left(0, D_{Z}\right),
$$

onde

$$
D_{Z}=\phi \exp [(k-2) \alpha] \Sigma_{z}^{-1}
$$

Usando o Lema 3.2 , sob $H_{0}$, temos que $\hat{\theta}_{X} \stackrel{\mathrm{p}}{\longrightarrow} \theta_{0}$, onde $\hat{\theta}_{X}$ é o EMV "naive". Pelo resultado mostrado em Stefanski (1985) temos que, sob $H_{0}$,

$$
n^{1 / 2}\left(\hat{\theta}_{X}-\theta_{0}\right) \stackrel{\mathcal{D}}{\longrightarrow} N\left(0, H^{-1}\left(\theta_{0}\right) G\left(\theta_{0}\right) H^{-1}\left(\theta_{0}\right)\right)
$$

onde

$$
H\left(\theta_{0}\right)=E\left[\dot{q}(\alpha)\left(1, x^{\prime}\right)^{\prime}\left(1, x^{\prime}\right)\right]=E[\dot{q}(\alpha)]\left(\begin{array}{cc}
1 & \mu^{\prime} \\
\mu & \Sigma_{z}+\Sigma_{u}+\mu \mu^{\prime}
\end{array}\right)
$$

e

$$
G\left(\theta_{0}\right)=E\left[q^{2}(\alpha)\left(1, x^{\prime}\right)^{\prime}\left(1, x^{\prime}\right)\right]=E\left[q^{2}(\alpha)\right]\left(\begin{array}{cc}
1 & \mu^{\prime} \\
\mu & \Sigma_{z}+\Sigma_{u}+\mu \mu^{\prime}
\end{array}\right),
$$

onde $\dot{q}(\alpha)$ é dada por $(3.68)$ e $q^{2}(\alpha)=\frac{1}{\phi^{2}} \exp [2(1-k) \alpha][y-\exp (\alpha)]^{2}$. Assim,

$$
H^{-1}\left(\theta_{0}\right) G\left(\theta_{0}\right) H^{-1}\left(\theta_{0}\right)=\{E[\dot{q}(\alpha)]\}^{-2} E\left[q^{2}(\alpha)\right]\left(\begin{array}{cc}
1+\mu^{\prime}\left(\Sigma_{z}+\Sigma_{u}\right)^{-1} \mu & -\mu^{\prime}\left(\Sigma_{z}+\Sigma_{u}\right)^{-1} \\
-\left(\Sigma_{z}+\Sigma_{u}\right)^{-1} \mu & \left(\Sigma_{z}+\Sigma_{u}\right)^{-1}
\end{array}\right),
$$

sendo $E\left[q^{2}(\alpha)\right]=\frac{1}{\phi} \exp [(2-k) \alpha]$, temos que, sob $H_{0}$,

$$
n^{1 / 2} \widehat{\beta}_{X} \stackrel{\mathcal{D}}{\longrightarrow} N\left(0, D_{X}\right)
$$

onde

$$
D_{X}=\phi \exp [(k-2) \alpha]\left(\Sigma_{z}+\Sigma_{u}\right)^{-1} .
$$

Seja $\widehat{\theta}_{c}$ o estimador do escore corrigido e consideremos em princípio que $\Sigma_{u}$ é conhecida. Na Seção 2.3 do Capítulo 2 foi mostrado que, sob $H_{0}, \widehat{\theta}_{c} \stackrel{\mathrm{p}}{\longrightarrow} \theta_{0} \mathrm{e}$

$$
n^{1 / 2}\left(\hat{\theta}_{c}-\theta_{0}\right) \stackrel{\mathcal{D}}{\longrightarrow} N\left(0, \Lambda^{-1}\left(\theta_{0}\right) \Gamma\left(\theta_{0}\right) \Lambda^{-1}\left(\theta_{0}\right)\right),
$$


onde

$$
\Lambda\left(\theta_{0}\right)=\frac{1}{n} \sum_{i=1}^{n} E\left[-\frac{\partial U_{i}^{*}}{\partial \theta}\left(\theta_{0}\right)\right] \quad \text { e } \quad \Gamma\left(\theta_{0}\right)=\frac{1}{n} \sum_{i=1}^{n} E\left[U_{i}^{*}\left(\theta_{0}\right) U_{i}^{*}\left(\theta_{0}\right)^{\prime}\right] .
$$

De (3.59) e (3.60), temos que

$$
\frac{\partial U_{i}^{*}}{\partial \theta}\left(\theta_{0}\right)=\frac{1}{\phi}\left[(1-k) \exp [(1-k) \alpha] y_{i}-(2-k) \exp [(2-k) \alpha]\left(\begin{array}{cc}
1 & x_{i}^{\prime} \\
x_{i} & x_{i} x_{i}^{\prime}-\Sigma_{u}
\end{array}\right)\right.
$$

e

$$
\begin{aligned}
U_{i}^{*}\left(\theta_{0}\right) U_{i}^{*}\left(\theta_{0}\right)^{\prime}= & \frac{1}{\phi}\left[\exp [2(1-k) \alpha] y_{i}^{2}-2 \exp [(3-k) \alpha] y_{i}\right. \\
& +\exp [2(2-k) \alpha]\left(\begin{array}{cc}
1 & x_{i}^{\prime} \\
x_{i} & x_{i} x_{i}^{\prime}
\end{array}\right)
\end{aligned}
$$

de modo que

$$
\grave{\Lambda}\left(\theta_{0}\right)=\frac{1}{\phi} \exp [(2-k) \alpha]\left(\begin{array}{cc}
1 & \mu^{\prime} \\
\mu & \Sigma_{z}+\mu \mu^{\prime}
\end{array}\right)
$$

e

$$
\Gamma\left(\theta_{0}\right)=\frac{1}{\phi} \exp [(2-k) \alpha]\left(\begin{array}{cc}
1 & \mu^{\prime} \\
\mu & \Sigma_{z}+\Sigma_{u}+\mu \mu^{\prime}
\end{array}\right) .
$$

Assim,

$$
\begin{aligned}
& \Lambda^{-1}\left(\theta_{0}\right) \Gamma\left(\theta_{0}\right) \Lambda^{-1}\left(\theta_{0}\right) \\
& =\phi \exp [(k-2) \alpha]\left(\begin{array}{cc}
1+\mu^{\prime} \Sigma_{z}^{-1} \mu+\mu^{\prime} \Sigma_{z}^{-1} \Sigma_{u} \Sigma_{z}^{-1} \mu & -\mu^{\prime} \Sigma_{z}^{-1}\left(I_{p}+\Sigma_{u} \Sigma_{z}^{-1}\right) \\
-\left(I_{p}+\Sigma_{z}^{-1} \Sigma_{u}\right) \Sigma_{z}^{-1} \mu & \left(I_{p}+\Sigma_{z}^{-1} \Sigma_{u}\right) \Sigma_{z}^{-1}
\end{array}\right) .
\end{aligned}
$$

Portanto, sob $H_{0}$,

$$
n^{1 / 2} \widehat{\beta}_{c} \stackrel{\mathcal{D}}{\longrightarrow} N\left(0, D_{c}\right),
$$

$\operatorname{com} D_{c}=\phi \exp [(k-2) \alpha]\left(I_{s}+\Sigma_{z}^{-1} \Sigma_{u}\right) \Sigma_{z}^{-1}$.

No caso em que $\Sigma_{u}$ é desconhecida, na Seção 2.4 do Capítulo 2 foi provado que a matriz de covariâncias assintótica de $\widehat{\theta}_{c}$ é dada por

$$
\Lambda^{-1}\left(\theta_{0}\right)\left\{\Gamma\left(\theta_{0}\right)-B\left(\theta_{0}\right) A^{-1}\left(\theta_{0}\right) D\left(\theta_{0}\right)-D\left(\theta_{0}\right)^{\prime} A^{-1}\left(\theta_{0}\right) B\left(\theta_{0}\right)^{\prime}+B\left(\theta_{0}\right) \operatorname{Var}(\widehat{\gamma}) B\left(\theta_{0}\right)^{\prime}\right\} \Lambda^{-1}\left(\theta_{0}\right)
$$


onde $\gamma=\operatorname{Vech}\left(\Sigma_{u}\right), \hat{\gamma}$ é um estimador consistente de $\gamma$ e $B\left(\theta_{0}\right)=\frac{1}{n} \sum_{i=1}^{n} E\left[\frac{\partial U_{i}^{*}}{\partial \gamma}\left(\theta_{0}\right)\right]$. A partir de (3.59) e (3.60), temos que

$$
\begin{aligned}
\frac{\partial U_{i \alpha}^{*}(\theta)}{\partial \Sigma_{u}}= & \frac{1}{2 \phi}\left\{-\exp \left[(1-k)\left(\alpha+\beta^{\prime} x_{i}\right)-\xi_{u}\right] y_{i}(1-k)^{2}\right. \\
& \left.+\exp \left[(2-k)\left(\alpha+\beta^{\prime} x_{i}\right)-\gamma_{u}\right](2-k)^{2}\right\} \beta \beta^{\prime}
\end{aligned}
$$

e

$$
\begin{aligned}
\frac{\partial U_{i \beta}^{*}(\theta)}{\partial \Sigma_{u}}= & \frac{1}{\phi}\left\{-\exp \left[(1-k)\left(\alpha+\beta^{\prime} x_{i}\right)-\xi_{u}\right] y_{i}(1-k)\right. \\
& \left.+\exp \left[(2-k)\left(\alpha+\beta^{\prime} x_{i}\right)-\gamma_{u}\right](2-k)\right\}\left(\beta^{\prime} \otimes \operatorname{Vec} I_{s}\right) \\
& +\frac{1}{2 \phi}\left\{-\exp \left[(1-k)\left(\alpha+\beta^{\prime} x_{i}\right)-\xi_{u}\right]\left[x_{i}-(1-k) \Sigma_{u} \beta\right] y_{i}(1-k)^{2} \otimes I_{s}\right. \\
& \left.+\exp \left[(2-k)\left(\alpha+\beta^{\prime} x_{i}\right)-\gamma_{u}\right]\left[x_{i}-(2-k) \Sigma_{u} \beta\right](2-k)^{2} \otimes I_{s}\right\} \beta \beta^{\prime},
\end{aligned}
$$

onde $\otimes$ denota o produto de Kronecker entre matrizes e Vec $I_{s}$ é o vetor dado por $\operatorname{Vec} I_{s}=$ $\left(e_{1}, e_{2}, \ldots, e_{s}\right)^{\prime}$, com $e_{i}$ denotando a $i$-ésima linha da matriz identidade $I_{s}$.

Portanto, $\frac{\partial U_{i \alpha}^{*}}{\partial \Sigma_{u}}\left(\theta_{0}\right)=0 \mathrm{e} \frac{\partial U_{i \beta}^{*}}{\partial \Sigma_{u}}\left(\theta_{0}\right)=0$, de modo que $\frac{\partial U_{i \alpha}^{*}}{\partial \gamma}\left(\theta_{0}\right)=0 \mathrm{e} \frac{\partial U_{i \beta}^{*}}{\partial \gamma}\left(\theta_{0}\right)=0$. Assim, $B\left(\theta_{0}\right)=0$ e a matriz de covariâncias assintótica de $\hat{\theta}_{c}$ sob $H_{0}$ é igual a $\Lambda^{-1}\left(\theta_{0}\right) \Gamma\left(\theta_{0}\right) \Lambda^{-1}\left(\theta_{0}\right)$. Conseqüentemente, a estatística de Wald corrigida é igual a $W_{c}$, dada em (3.5) com $\widehat{\Sigma}_{u}$ no lugar de $\Sigma_{u}$.

(b) $\operatorname{Sob} \beta=\beta^{(n)}$,

$$
\begin{array}{ll}
n^{1 / 2} \widehat{\beta}_{Z} & \stackrel{\mathcal{D}}{\longrightarrow} N\left(\Delta, D_{Z}\right), \\
n^{1 / 2} \widehat{\beta}_{X} & \stackrel{\mathcal{D}}{\longrightarrow} N\left(R \Delta, D_{X}\right),
\end{array}
$$

desde que, se $\beta=\beta^{(n)}, \widehat{\beta}_{X} \stackrel{\mathrm{p}}{\longrightarrow} \beta^{*(n)}$, com $\beta^{*(n)}=R \beta^{(n)}$ pelo Lema 3.2 , e

$$
n^{1 / 2} \widehat{\beta}_{c} \stackrel{\mathcal{D}}{\longrightarrow} N\left(\Delta, D_{c}\right) .
$$

Proposição 3.6 Dadas as suposições (i) - (v) e (3.61),

$$
\operatorname{ARE}\left(n^{1 / 2} \widehat{\beta}_{c}: n^{1 / 2} \widehat{\beta}_{Z}\right) \leq 1, \operatorname{ARE}\left(n^{1 / 2} \widehat{\beta}_{X}: n^{1 / 2} \widehat{\beta}_{Z}\right) \leq 1 \quad e \quad \operatorname{ARE}\left(n^{1 / 2} \widehat{\beta}_{X}: n^{1 / 2} \widehat{\beta}_{c}\right)=1
$$


Prova: Denotando por $\widehat{D}_{Z}, \widehat{D}_{X}$ e $\widehat{D}_{c}$ os estimadores de $D_{Z}, D_{X}$ e $D_{c}$, respectivamente, sob a seqüência de alternativas

$$
\begin{aligned}
W_{Z} & =n \widehat{\beta}_{Z}^{\prime} \widehat{D}_{Z}^{-1} \widehat{\beta}_{Z} \stackrel{\mathcal{D}}{\longrightarrow} \chi^{2}\left(M_{Z}\right), \\
W_{X} & =n \widehat{\beta}_{X}^{\prime} \widehat{D}_{X}^{-1} \widehat{\beta}_{X} \stackrel{\mathcal{D}}{\longrightarrow} \chi^{2}\left(M_{X}\right), \\
W_{c} & =n \widehat{\beta}_{c}^{\prime} \widehat{D}_{c}^{-1} \widehat{\beta}_{c} \stackrel{\mathcal{D}}{\longrightarrow} \chi^{2}\left(M_{c}\right),
\end{aligned}
$$

onde

$$
\begin{aligned}
M_{Z} & =\Delta^{\prime} D_{Z}^{-1} \Delta=d \Delta^{\prime} \Sigma_{z} \Delta, \\
M_{X} & =\Delta^{\prime} R^{\prime} \widehat{D}_{X}^{-1} R \Delta=d \Delta^{\prime} \Sigma_{z}\left(\Sigma_{z}+\Sigma_{u}\right)^{-1} \Sigma_{z} \Delta \\
M_{c} & =\Delta^{\prime} \widehat{D}_{c}^{-1} \Delta=d \Delta^{\prime} \Sigma_{z}\left(\Sigma_{z}+\Sigma_{u}\right)^{-1} \Sigma_{z} \Delta .
\end{aligned}
$$

Portanto, como

$$
\begin{aligned}
M_{Z}-M_{c} & =M_{Z}-M_{X}=d \Delta^{\prime}\left\{\Sigma_{z}-\Sigma_{z}\left(\Sigma_{z}+\Sigma_{u}\right)^{-1} \Sigma_{z}\right\} \Delta \\
& =d \Delta^{\prime} \Sigma_{z}\left(\Sigma_{z}+\Sigma_{u}\right)^{-1} \Sigma_{u} \Delta \geq 0
\end{aligned}
$$

segue que $\operatorname{ARE}\left(n^{1 / 2} \widehat{\beta}_{c}: n^{1 / 2} \widehat{\beta}_{Z}\right)=\operatorname{ARE}\left(n^{1 / 2} \widehat{\beta}_{X}: n^{1 / 2} \widehat{\beta}_{Z}\right) \leq 1, \operatorname{e} \operatorname{ARE}\left(n^{1 / 2} \widehat{\beta}_{X}: n^{1 / 2} \widehat{\beta}_{c}\right)=$ $\frac{M_{c}}{M_{X}}=1$.

O corolário seguinte é imediato.

Corolário 3.2 Se $s=1$, então

$$
\operatorname{ARE}\left(n^{1 / 2} \widehat{\beta}_{X}: n^{1 / 2} \widehat{\beta}_{Z}\right)=\operatorname{ARE}\left(n^{1 / 2} \widehat{\beta}_{c}: n^{1 / 2} \widehat{\beta}_{Z}\right)=\operatorname{corr}(z, x)^{2}=\frac{\sigma_{z}^{2}}{\sigma_{z}^{2}+\sigma_{u}^{2}} .
$$

Observação: Os resultados obtidos nesta seção também valem para o modelo de regressão linear normal. Pode ser mostrado que $\hat{\theta}_{X} \stackrel{\mathrm{p}}{\longrightarrow} \theta^{*}$, onde $\theta^{*}=\left(\alpha^{*}, \beta^{*^{\prime}}\right)^{\prime} \operatorname{com} \beta^{*}=R \beta$ e $\alpha^{*}=\alpha+\mu^{\prime}\left(I_{p}-R\right) \beta$, e $d$ na Proposição 3.5 é dado por $d=\phi$. 
Dado que a perda em eficiência medida em termos assintóticos não mostra necessariamente a perda real em pequenas amostras, a seguir apresentamos um estudo de simulação para comparar o comportamento dos três testes $W_{Z}, W_{X}$ e $W_{c}$ em pequenas amostras.

\subsubsection{Um estudo de simulação}

Com o objetivo de comparar o poder empírico dos testes $W_{Z}, W_{X}$ e $W_{c}$, um estudo de simulação foi realizado, baseado num modelo de regressão exponencial.

A verdadeira covariável $z_{i}$ foi gerada como uma normal padrão, i.e., $z_{i} \sim N(0,1)$.

A covariável observada $x_{i}$ foi gerada, condicionada na verdadeira covariável $z_{i}$, segundo o modelo $x_{i}=z_{i}+u_{i}$, onde $u_{i} \sim N\left(0, \sigma_{u}^{2}\right)$.

A variável $y_{i}$, condicionada em $z_{i}$ foi gerada como uma variável aleatória exponencial com parâmetro $\exp \left(\alpha+\beta z_{i}\right)$, com $\alpha=0$. A hipótese nula de interesse foi $\beta=0$.

1.000 replicações foram geradas para cada simulação, combinando os tamanhos amostrais $n=30$ e 50 com os valores de $\sigma_{u}^{2}=0.1,0.3$ e 0.5 .

Um ajuste foi feito usando as distribuições empíricas das estatísticas, obtidas da simulação, de forma que o tamanho empírico corresponda ao de $5 \%$.

Na Figura 3.2 apresentamos as curvas de poder dos testes obtidas para as diferentes combinações de tamanho amostral e variância do erro.

$W_{X}$ e $W_{c}$ perdem poder quando a variância do erro cresce. $\mathrm{O}$ poder das estatísticas $W_{X}$ e $W_{c}$ parece similar. Contudo, quando $\sigma_{u}^{2}$ cresce, o teste de Wald "naive" $W_{X}$ parece melhor do que o teste de Wald corrigido $W_{c}$. 
a)

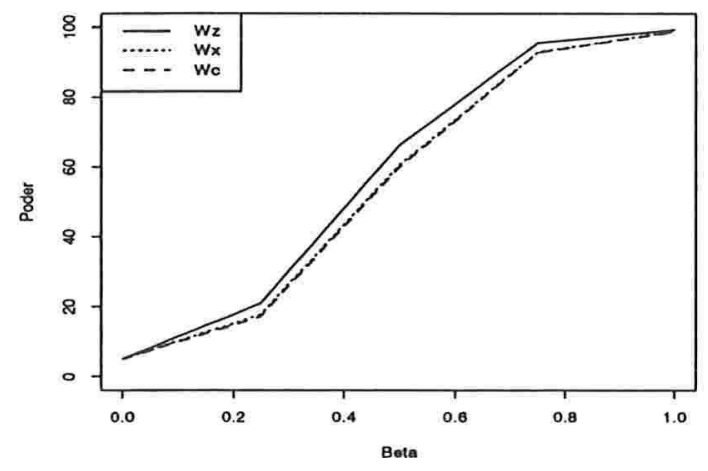

c)

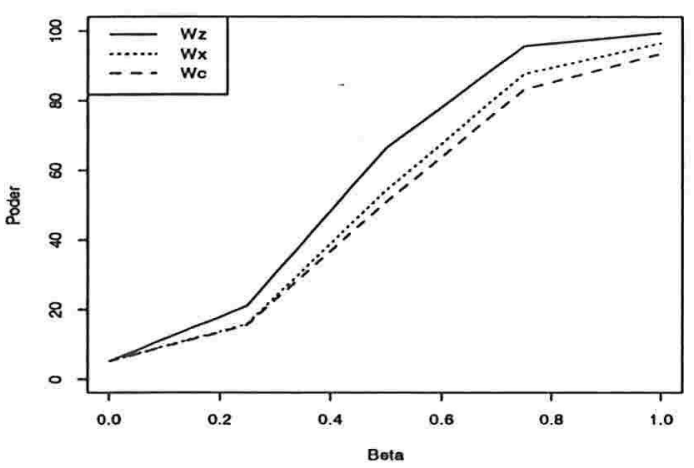

e)

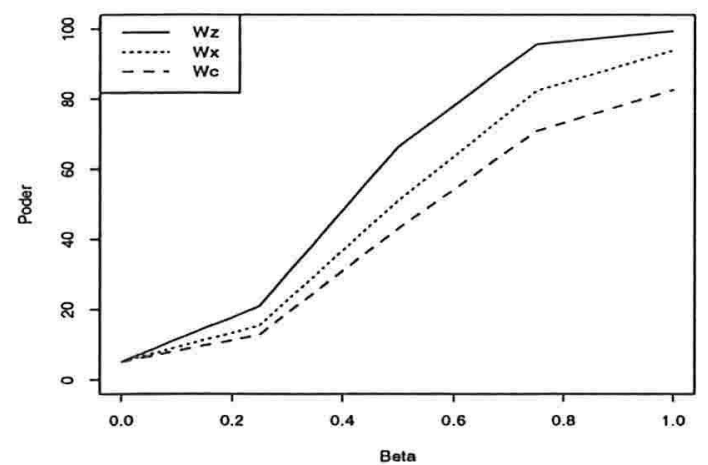

b)

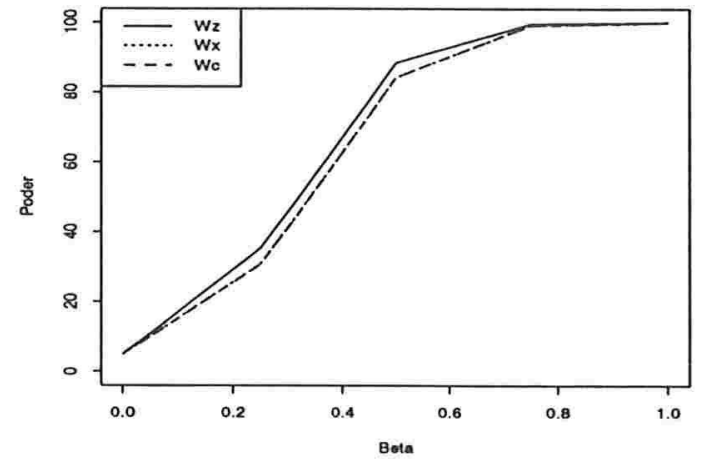

d)

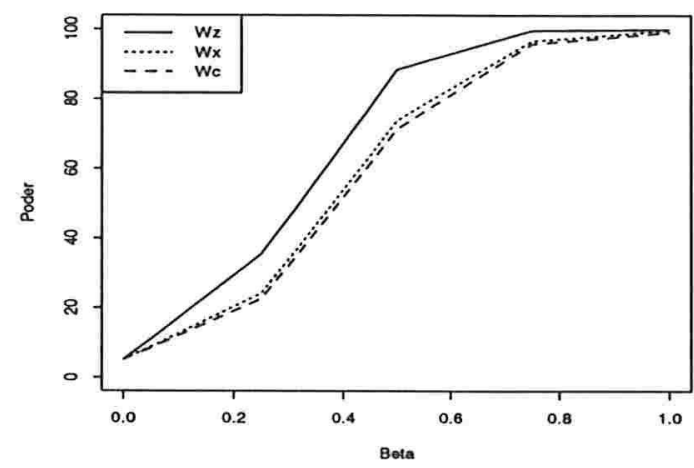

f)

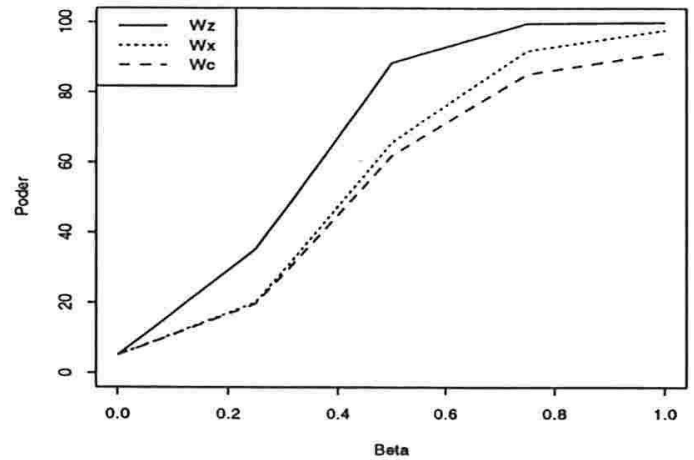

Figura 3.2: Curvas de poder dos testes $W_{Z}, W_{X}$ e $W_{c}$. a) $\sigma_{u}^{2}=0.1, n=30$, b) $\sigma_{u}^{2}=0.1, n=$ 50, c) $\sigma_{u}^{2}=0.3, n=30$, d) $\sigma_{u}^{2}=0.3, n=50$, e) $\sigma_{u}^{2}=0.5, n=30$, f) $\sigma_{u}^{2}=0.5, n=50$. 


\section{Capítulo 4}

\section{O modelo de calibração comparativa}

\subsection{Introdução}

Neste capítulo estudamos os modelos de calibração comparativa funcional e estrutural, utilizando a metodologia apresentada no Capítulo 2. Estes modelos são freqüentemente usados para comparar instrumentos ou métodos de medição. O termo "método" ou "instrumento" (de medição) refere-se a diferentes formas de medição da mesma quantidade desconhecida.

Suponha que dispomos de $p+1(p \geq 1)$ instrumentos para medir uma característica ou resposta $z$, num grupo de $n$ unidades.

Um modelo freqüentemente adotado na literatura (veja Barnett, 1969) para este tipo de situações é dado por

$$
\begin{aligned}
& y_{i}=\alpha+\beta z_{i}+\varepsilon_{i} \\
& x_{i}=z_{i}+u_{i}, \quad i=1, \ldots, n,
\end{aligned}
$$

onde $y_{i}=\left(y_{i 1}, \ldots, y_{i p}\right)^{\prime}, \varepsilon_{i}=\left(\varepsilon_{i 1}, \ldots, \varepsilon_{i p}\right)^{\prime}, i=1, \ldots, n$, são vetores aleatórios de dimensão $p \times 1$ e $\alpha=\left(\alpha_{1}, \ldots, \alpha_{p}\right)^{\prime}$ e $\beta=\left(\beta_{1}, \ldots, \beta_{p}\right)^{\prime}$ são os parâmetros sob os quais desejamos fazer inferências.

- $z_{i}$ representa o verdadeiro valor da característica de interesse na unidade $i, i=$ $1, \ldots, n$. 
- $y_{i k}$ corresponde à medida fornecida pelo instrumento $k$ para a característica da unidade $i, k=1, \ldots, p, i=1, \ldots, n$.

- $\alpha_{k}$ e $\beta_{k}$ são os vícios aditivo e multiplicativo do instrumento $k$, respectivamente, $k=1, \ldots, p$.

A equação (4.2) considera que um dos instrumentos $(p+1)$ mede a quantidade desconhecida $z_{i}$ correspondente ao indivíduo $i$ sem vício. Na literatura (Barnett, 1969) este instrumento é chamado de instrumento de referência ou padrão.

É assumido que $\varepsilon_{i} \stackrel{\mathrm{iid}}{\sim} N(0, \Sigma), \operatorname{com} \Sigma=\operatorname{Diag}\left(\sigma_{1}^{2}, \ldots, \sigma_{p}^{2}\right)$ e $u_{i} \stackrel{\mathrm{iid}}{\sim}\left(0, \sigma_{u}^{2}\right), i=1, \ldots, n$.

Este modelo é reconhecido como um caso especial de um modelo de regressão linear com erros nas variáveis (Kendall e Stuart, 1979, e Fuller, 1987).

Se as $z_{i}$ 's são constantes desconhecidas, o modelo descrito por (4.1) e (4.2) é um modelo linear funcional com erros nas variáveis. Quando as $z_{i}$ 's são uma amostra aleatória de uma população com média $\mu$ e variância $\sigma_{z}^{2}$, não correlacionadas com os $\varepsilon_{i k}$, o modelo (4.1) - (4.2) é denominado modelo linear estrutural com erros nas variáveis.

Quando $p=1$, o modelo (4.1) - (4.2) corresponde ao modelo de regressão linear simples com erros nas variáveis, extensivamente discutido na literatura, veja Fuller (1987) e referências ali citadas.

Para $p \geq 2$, o modelo estrutural sob normalidade dos $\varepsilon_{i}$ 's e $u_{i}$ 's tem sido estudado por vários autores. Para $p>2$, os EMV não têm forma explícita. Devido a isso, Barnett (1969) propõe estimadores usando o método dos momentos. Para $p=2$, consegue estimativas explícitas dos parâmetros do modelo e da matriz de covariâncias assintótica dos estimadores. Carter (1981) utiliza estimação de máxima verossimilhança restrita. Theobald e Mallinson (1978) reparametrizam o modelo proposto por Barnett, na forma de análise de fatores e usam pacotes desenvolvidos para esse tipo de modelos, para obter os EMV. Fuller (1987) dá um procedimento iterativo para obter os EMV e sua matriz 
de covariâncias assintótica. Galea-Rojas (1995) propõe uma forma alternativa para a obtenção dos EMV, via algoritmo EM (Dempster et al., 1977), no caso normal e $t$-elíptico. Kimura (1992) estuda o modelo funcional normal e encontra os EMV utilizando um tipo de algoritmo EM. Galea-Rojas (1995), baseado em alguns resultados de Mak (1982), complementa o trabalho de Kimura, apresentando os EMV, como também sua distribuição assintótica.

Os trabalhos antes citados estudam separadamente um dos dois tipos de modelos (estrutural ou funcional). No entanto, a metodologia do escore corrigido pode ser aplicada para tratar simultaneamente ambos os tipos de modelos.

Por outro lado, Gleser (1983) também mostra, no caso de modelos lineares de regressão, que não é necessário tratar os dois tipos de modelos separadamente. Consistência e normalidade assintótica de estimadores num modelo funcional implicam em resultados similares para tais estimadores num modelo estrutural associado.

É sabido (Gleser, 1983) que, identificabilidade do modelo (4.1) - (4.2), é condição necessária para a existência de estimadores consistentes dos parâmetros. Duas condições que tornam o modelo (4.1) - (4.2) identificável, serão consideradas neste capítulo: $\sigma_{u}^{2}$ conhecida e as razões de variâncias $\lambda_{k}=\frac{\sigma_{k}^{2}}{\sigma_{u}^{2}}, k=1, \ldots, p$, conhecidas. O método do escore corrigido será aplicado para a obtenção de estimadores consistentes e assintoticamente normais no modelo funcional em ambos os casos. Não assumiremos normalidade do erro $u_{i}$, exigindo apenas existência do quarto momento finito.

\subsection{O caso $\sigma_{u}^{2}$ conhecida}

Considere o modelo funcional

$$
\begin{aligned}
y_{i} & =\alpha+\beta z_{i}+\varepsilon_{i}, \\
x_{i} & =z_{i}+u_{i}, \quad i=1, \ldots, n,
\end{aligned}
$$


onde $y_{i}=\left(y_{i 1}, \ldots, y_{i p}\right)^{\prime}, \varepsilon_{i}=\left(\varepsilon_{i 1}, \ldots, \varepsilon_{i p}\right)^{\prime}, \alpha=\left(\alpha_{1}, \ldots, \alpha_{p}\right)^{\prime}$ e $\beta=\left(\beta_{1}, \ldots, \beta_{p}\right)^{\prime}$, com $\varepsilon_{i} \stackrel{\text { iid }}{\sim} N(0, \Sigma), \Sigma=\operatorname{Diag}\left(\sigma_{1}^{2}, \ldots, \sigma_{p}^{2}\right)$ e $u_{i} \stackrel{\text { iid }}{\sim}\left(0, \sigma_{u}^{2}\right), \sigma_{u}^{2}$ conhecida e $E\left(u_{i}^{4}\right)<\infty$.

\subsubsection{O estimador do escore corrigido, propriedades assintóticas}

Os $3 p$ parâmetros estruturais do modelo são dados por $\theta=\left(\alpha^{\prime}, \beta^{\prime}, \phi^{\prime}\right)^{\prime}$, onde $\phi=\left(\sigma_{1}^{2}, \ldots, \sigma_{p}^{2}\right)^{\prime}$ e $z_{1}, \ldots, z_{n}$ representam os parâmetros incidentais.

A log-verossimilhança "não observada" (i.e. baseada nos verdadeiros valores das covariáveis $z_{i}$ ) é dada por

$$
\ell(\theta ; Z, Y)=\sum_{i=1}^{n} \ell_{i}\left(\theta ; z_{i}, y_{i}\right)
$$

onde

$$
\ell_{i}\left(\theta ; z_{i}, y_{i}\right)=-\frac{p}{2} \log (2 \pi)-\frac{1}{2} \log |\Sigma|-\frac{1}{2}\left(y_{i}-\alpha-\beta z_{i}\right)^{\prime} \Sigma^{-1}\left(y_{i}-\alpha-\beta z_{i}\right)
$$

$i=1, \ldots, n$.

Portanto, o escore "não observado" é dado por

$$
U(\theta ; Z, Y)=\sum_{i=1}^{n} U_{i}\left(\theta ; z_{i}, y_{i}\right)
$$

onde $U_{i}=\left(U_{i \alpha}^{\prime}, U_{i \beta}^{\prime}, U_{i \phi}^{\prime}\right)^{\prime}$, com

$$
\begin{aligned}
& U_{i \alpha}(\theta)=\frac{\partial \ell_{i}(\theta)}{\partial \alpha}=\Sigma^{-1}\left(y_{i}-\alpha-\beta z_{i}\right) \\
& U_{i \beta}(\theta)=\frac{\partial \ell_{i}(\theta)}{\partial \beta}=\Sigma^{-1}\left(y_{i}-\alpha-\beta z_{i}\right) z_{i} \\
& U_{i \phi}(\theta)=\frac{\partial \ell_{i}(\theta)}{\partial \phi}=-\frac{1}{2} \Sigma^{-1} 1_{p}+\frac{1}{2} \Sigma^{-2}\left[\left(y_{i}-\alpha-\beta z_{i}\right) *\left(y_{i}-\alpha-\beta z_{i}\right)\right],
\end{aligned}
$$

onde $1_{p}$ é o vetor $p \times 1$ com todos os elementos iguais a 1 e $*$ denota o produto de Hadamard entre vetores.

Considerando $U_{i}^{*}=\left(U_{i \alpha}^{*^{\prime}}, U_{i \beta}^{*^{\prime}}, U_{i \phi}^{*^{\prime}}\right)^{\prime}$, onde

$$
\begin{aligned}
& U_{i \alpha}^{*}(\theta)=\Sigma^{-1}\left(y_{i}-\alpha-\beta x_{i}\right) \\
& U_{i \beta}^{*}(\theta)=\Sigma^{-1}\left[\left(y_{i}-\alpha-\beta x_{i}\right) x_{i}+\beta \sigma_{u}^{2}\right] \\
& U_{i \phi}^{*}(\theta)=-\frac{1}{2} \Sigma^{-1} 1_{p}+\frac{1}{2} \Sigma^{-2}\left[\left(y_{i}-\alpha-\beta x_{i}\right) *\left(y_{i}-\alpha-\beta x_{i}\right)-(\beta * \beta) \sigma_{u}^{2}\right],
\end{aligned}
$$


segue que

$$
E\left[U_{i}^{*}\left(\theta, x_{i}, y_{i}\right) \mid z_{i}, y_{i}\right]=U_{i}\left(\theta ; z_{i}, y_{i}\right), \quad i=1, \ldots, n
$$

De modo que, segundo a Definição $2.1, U^{*}(\theta ; X, Y)=\sum_{i=1}^{n} U_{i}^{*}\left(\theta ; x_{i}, y_{i}\right)$ é um escore corrigido.

Note que, neste caso, sendo

$$
\begin{aligned}
\ell_{i}^{*}\left(\theta, x_{i}, y_{i}\right)= & -\frac{p}{2} \log (2 \pi)-\frac{1}{2} \log |\Sigma|-\frac{1}{2}\left(y_{i}-\alpha-\beta x_{i}\right)^{\prime} \Sigma^{-1}\left(y_{i}-\alpha-\beta x_{i}\right) \\
& +\frac{1}{2} \beta^{\prime} \Sigma^{-1} \beta \sigma_{u}^{2},
\end{aligned}
$$

segue que

$$
E\left[\ell_{i}^{*}\left(\theta ; x_{i}, y_{i}\right) \mid z_{i}, y_{i}\right]=\ell_{i}\left(\theta ; z_{i}, y_{i}\right), \quad i=1, \ldots, n
$$

e

$$
\frac{\partial \ell_{i}^{*}(\theta)}{\partial \theta}=U_{i}^{*}(\theta), \quad i=1, \ldots, n .
$$

Resolvendo a equação $U^{*}(\theta ; X, Y)=0$, obtemos os estimadores do escore corrigido,

$$
\begin{aligned}
\widehat{\alpha} & =\bar{y}-\widehat{\beta} \bar{x} \\
\widehat{\beta} & =\frac{S_{x y}}{S_{x x}-\sigma_{u}^{2}}, \text { se } S_{x x}>\sigma_{u}^{2} \\
\mathrm{e} \quad \hat{\sigma}_{k}^{2} & =S_{y_{k} y_{k}}-\widehat{\beta}_{k} S_{x y_{k}}, \text { se } S_{x x} \geq \frac{S_{x y_{k}}^{2}}{S_{x x}-\sigma_{u}^{2}}, k=1, \ldots, p,
\end{aligned}
$$

onde

$$
\begin{aligned}
& \bar{x}=\frac{1}{n} \sum_{i=1}^{n} x_{i}, \quad \bar{y}=\frac{1}{n} \sum_{i=1}^{n} y_{i}, \quad \bar{y}_{k}=\frac{1}{n} \sum_{i=1}^{n} y_{i k}, \\
& S_{x x}=\frac{1}{n} \sum_{i=1}^{n}\left(x_{i}-\bar{x}\right)^{2}, \quad S_{x y}=\frac{1}{n} \sum_{i=1}^{n}\left(x_{i}-\bar{x}\right)\left(y_{i}-\bar{y}\right), \\
& S_{x y_{k}}=\frac{1}{n} \sum_{i=1}^{n}\left(x_{i}-\bar{x}\right)\left(y_{i k}-\bar{y}_{k}\right), \quad S_{y_{k} y_{k}}=\frac{1}{n} \sum_{i=1}^{n}\left(y_{i k}-\bar{y}_{k}\right)^{2} .
\end{aligned}
$$

De (4.4) e (4.5) segue que

$$
\begin{aligned}
& \widehat{\alpha}_{k}=\bar{y}_{k}-\widehat{\beta}_{k} \bar{x} \\
& \widehat{\beta}_{k}=\frac{S_{x y_{k}}}{S_{x x}-\sigma_{u}^{2}}, \quad k=1, \ldots, p,
\end{aligned}
$$


de modo que as componentes $k$-ésimas dos estimadores $\widehat{\alpha}$ e $\widehat{\beta}$ correspondem aos EMV de um modelo de regressão linear simples com erros nas variáveis, com $\sigma_{u}^{2}$ conhecida e $y_{k}$ como variável dependente (veja, por exemplo, Fuller, 1987).

Depois de alguma álgebra, as matrizes $\bar{\Lambda}_{n}(\theta)$ e $\bar{\Gamma}_{n}(\theta)$ definidas em (2.8) e (2.9), respectivamente, são dadas por

$$
\bar{\Lambda}_{n}(\theta)=\left(\begin{array}{cc}
A \otimes \Sigma^{-1} & 0 \\
0 & \frac{1}{2} \Sigma^{-2}
\end{array}\right), \quad \bar{\Gamma}_{n}(\theta)=\left(\begin{array}{cc}
\Gamma_{1} & \Gamma_{2} \\
\Gamma_{2}^{\prime} & \Gamma_{3}
\end{array}\right),
$$

onde

$$
\begin{aligned}
& \Gamma_{1}=B \otimes \Sigma^{-2} \beta \beta^{\prime}+C \otimes \Sigma^{-1} \\
& \Gamma_{2}=D \otimes \Sigma^{-3} \beta(\beta * \beta)^{\prime}-E \otimes \Sigma^{-2} D(\beta * \beta), \\
& \Gamma_{3}=\frac{1}{4} \Sigma^{-4}(\beta * \beta)(\beta * \beta)^{\prime}\left(\mu_{4}-\sigma_{u}^{4}\right)+\frac{1}{2} \Sigma^{-2}+\sigma_{u}^{2} \Sigma^{-3} D(\beta * \beta),
\end{aligned}
$$

com

$$
\begin{aligned}
A & =\left(\begin{array}{cc}
1 & \bar{z} \\
\bar{z} & S_{z z}+\bar{z}^{2}
\end{array}\right), \\
B & =\left(\begin{array}{cc}
\bar{z} \sigma_{u}^{2}+\mu_{3} \\
\bar{z} \sigma_{u}^{2}+\mu_{3} & \sigma_{u}^{2}\left(S_{z z}+\bar{z}\right)^{2}+\mu_{4}+2 \bar{z} \mu_{3}-\sigma_{u}^{2}
\end{array}\right) \\
C & =\left(\begin{array}{cc}
1 & \bar{z} \\
\bar{z} & S_{z z}+\bar{z}^{2}+\sigma_{u}^{2}
\end{array}\right) \\
D & =-\frac{1}{2}\left(\mu_{3}, \bar{z} \mu_{3}+\mu_{4}-\sigma_{u}^{4}\right)^{\prime} \\
E & =\left(0, \sigma_{u}^{2}\right)^{\prime}
\end{aligned}
$$

sendo

$$
\begin{aligned}
& \bar{z}=\frac{1}{n} \sum_{i=1}^{n} z_{i}, \quad S_{z z}=\frac{1}{n} \sum_{i=1}^{n}\left(z_{i}-\bar{z}\right)^{2}, \\
& \mu_{3}=E\left(u_{i}^{3}\right) \quad \text { e } \quad \mu_{4}=E\left(u_{i}^{4}\right) ;
\end{aligned}
$$

$\mathrm{com} \otimes$ denotando o produto de Kronecker entre matrizes e $D(\beta * \beta)=\operatorname{Diag}\left(\beta_{1}^{2}, \ldots, \beta_{p}^{2}\right)$. 
As condições de regularidade C1 - C5 da Seção 2.3.1 implicam nas seguintes condições sobre o comportamento da seqüência de parâmetros incidentais $z_{1}, \ldots, z_{n}$ :

$$
\begin{aligned}
& 0<\liminf \frac{1}{n} \sum_{i=1}^{n}\left(z_{i}-\bar{z}\right)^{2} \leq \limsup \frac{1}{n} \sum_{i=1}^{n}\left(z_{i}-\bar{z}\right)^{2}<\infty, \\
& \lim _{n \rightarrow \infty} \frac{1}{n^{1+\frac{\gamma}{2}}} \sum_{i=1}^{n}\left|z_{i}\right|^{2+\gamma}=0,
\end{aligned}
$$

para algum $\gamma>0$. Por conveniência, simplesmente escrevemos $z_{i}$ para os verdadeiros valores das covariáveis, $z_{i 0}$. Como a seqüência $z_{1}, \ldots, z_{n}$ de parâmetros incidentais não é observável, nunca teremos certeza se as suposições (4.7) e (4.8) são válidas. Na literatura, todos os resultados assintóticos obtidos para o modelo funcional impõem algum tipo de restrição na seqüência $z_{1}, \ldots, z_{n}$. Com exceção de Mak (1982), a restrição mais comumente adotada e a existência dòs limites $\mu$ e $\nu^{2}$ tais que

$$
\lim _{n \rightarrow \infty} \bar{z}=\mu \quad \text { e } \quad \lim _{n \rightarrow \infty} S_{z z}=\nu^{2}
$$

(veja por exemplo, Gleser, 1983, e Cheng e Van Ness, 1994). Gleser (1983) usa a terminologia "quase-aleatório" para referir-se a um modelo funcional onde a seqüência de parâmetros incidentais verifica (4.9). O termo "quase-aleatório" tem a ver com o fato de que a seqüência $z_{1}, \ldots, z_{n}$, mesmo não sendo aleatoriamente gerada, tem algumas das propriedades (convergência do primeiro e segundo momentos amostrais) de uma seqüência de variáveis aleatórias i.i.d. Também é mostrado em Gleser (1983), para modelos lineares de regressão que, consistência e normalidade assintótica de uma seqüência de estimadores para um modelo funcional "quase-aleatório" implica em resultados análogos para tais estimadores, num modelo estrutural onde as $z_{i}$ 's são v.a.i.i.d. com distribuição $N\left(\mu, \nu^{2}\right)$.

Sob as suposições (4.7) e (4.8), mais fracas do que a existn̂cia dos limites $\mu$ e $\nu^{2}$, pode ser provada a consistência e normalidade assintótica do estimador do escore corrigido, para o modelo funcional com erro nas variáveis. Conseqüentemente, resultados assintóticos análogos para tais estimadores serão válidos para o modelo estrutural. 
Se a seqüência de parâmetros incidentais verifica (4.7) e (4.8), segue dos Teoremas

2.1 e 2.2 que o estimador $\hat{\theta}=\left(\widehat{\alpha}^{\prime}, \widehat{\beta}^{\prime}, \widehat{\phi}^{\prime}\right)^{\prime}$ definido por (4.4), (4.5) e (4.6) é consistente e assintoticamente normal, com vetor de médias $\theta_{0}=\left(\alpha_{0}^{\prime}, \beta_{0}^{\prime}, \phi_{0}^{\prime}\right)^{\prime}$ e matriz de covariâncias dada por $n^{-1} \Omega_{n}$, onde

$$
\Omega_{n}=\bar{\Lambda}_{n}^{-1} \bar{\Gamma}_{n} \bar{\Lambda}_{n}^{-1}=\left(\begin{array}{cc}
\Omega_{1} & \Omega_{2} \\
\Omega_{2}^{\prime} & \Omega_{3}
\end{array}\right)
$$

com

$$
\begin{aligned}
& \Omega_{1}=F \otimes \beta_{0} \beta_{0}^{\prime}+G \otimes \Sigma_{0}, \\
& \Omega_{2}=H \otimes \beta_{0}\left(\beta_{0} * \beta_{0}\right)^{\prime}+\left(K \otimes \Sigma_{0}\right) D\left(\beta_{0}\right), \\
& \Omega_{3}=\left(\beta_{0} * \beta_{0}\right)\left(\beta_{0} * \beta_{0}\right)^{\prime}\left(\mu_{4}-\sigma_{u}^{4}\right)+2 \Sigma_{0}^{2}+4 \sigma_{u}^{2} \Sigma_{0} D\left(\beta_{0} * \beta_{0}\right),
\end{aligned}
$$

sendo $\Sigma_{0}=\operatorname{Diag}\left(\sigma_{10}^{2}, \ldots, \sigma_{p 0}^{2}\right)$,

$$
F=\frac{1}{S_{z z}^{2}}\left(\begin{array}{cc}
S_{z z}^{2} \sigma_{u}^{2}+\bar{z}^{2} a-2 \bar{z} S_{z z} \mu_{3} & -\bar{z} a+S_{z z} \mu_{3} \\
-\bar{z} a+S_{z z} \mu_{3} & 1
\end{array}\right),
$$

onde

$$
\begin{aligned}
a & =S_{z z} \sigma_{u}^{2}+\mu_{4}-\sigma_{u}^{4} \\
G & =\frac{1}{S_{z z}^{2}}\left(\begin{array}{cc}
S_{z z}^{2}+\bar{z}^{2}\left(S_{z z}+\sigma_{u}^{2}\right) & -\bar{z}\left(S_{z z}+\sigma_{u}^{2}\right) \\
-\bar{z}\left(S_{z z}+\sigma_{u}^{2}\right) & S_{z z}+\sigma_{u}^{2}
\end{array}\right), \\
H & =\frac{1}{S_{z z}}\left(\begin{array}{c}
-S_{z z} \mu_{3}+\bar{z}\left(\mu_{4}-\sigma_{u}^{4}\right) \\
-\left(\mu_{4}-\sigma_{u}^{4}\right)
\end{array}\right), \\
K & =\frac{2 \sigma_{u}^{2}}{S_{z z}}\left(\begin{array}{c}
\bar{z} \\
-1
\end{array}\right) .
\end{aligned}
$$

No caso de um modelo estrutural com $z_{i} \stackrel{\text { iid }}{\sim} N\left(\mu, \nu^{2}\right)$, temos que $\frac{1}{n} \sum_{i=1}^{n} z_{i} \rightarrow \mu$ e $\frac{1}{n} \sum_{i=1}\left(z_{i}-\bar{z}\right)^{2} \rightarrow \nu^{2}$, quando $n \rightarrow \infty$. De modo que, a matriz de covariâncias assintótica é dada por $n^{-1} \Omega \operatorname{com} \Omega$ como $\Omega_{n}$ em (4.10) e (4.11), com $\mu$ e $\nu^{2}$ no lugar de $\bar{z}$ e $S_{z z}$, respectivamente, nas expressões $(4.12)-(4.15)$.

\subsubsection{O modelo de regressão linear simples}

Quando $p=1$, o modelo dado por (4.3) corresponde ao modelo de regressão linear simples com erros nas variáveis (veja, por exemplo, Cheng e Van Ness, 1994, para um resumo dos 
resultados existentes para ambos os tipos de modelos, funcional e estrutural, no caso normal).

Considere agora $\varepsilon_{i} \stackrel{\text { iid }}{\sim} N\left(0, \sigma_{\varepsilon}^{2}\right)$, de modo que os três parâmetros estruturais do modelo são dados por $\theta=\left(\alpha, \beta, \sigma_{\varepsilon}^{2}\right)^{\prime}$.

Os estimadores obtidos pelo método do escore corrigido são

$$
\begin{aligned}
\hat{\alpha} & =\bar{y}-\hat{\beta} \bar{x}, \\
\hat{\beta} & =\frac{S_{x y}}{S_{x x}-\sigma_{u}^{2}}, \text { se } S_{x x}>\sigma_{u}^{2}, \\
\hat{\sigma}_{\varepsilon}^{2} & =S_{y y}-\widehat{\beta} S_{x y}, \text { se } S_{x x} \geq \frac{S_{x y}^{2}}{S_{x x}-\sigma_{u}^{2}},
\end{aligned}
$$

onde

$$
S_{x y}=\frac{1}{n} \sum_{i=1}^{n}\left(x_{i}-\bar{x}\right)\left(y_{i}-\bar{y}\right) \quad \text { e } \quad S_{y y}=\frac{1}{n} \sum_{i=1}^{n}\left(y_{i}-\bar{y}\right)^{2} .
$$

É sabido (Cheng e Van Ness, 1994) que para o modelo funcional (4.3) com $p=1 \mathrm{e}$ $u_{i} \stackrel{\mathrm{iid}}{\sim} N\left(0, \sigma_{u}^{2}\right)$, os EMV de $\left(\alpha, \beta, \sigma_{\varepsilon}^{2}\right)$ são inconsistentes.

Os estimadores dados em (4.16) são consistentes e assintoticamente normais e coincidem com os estimadores obtidos pelo método dos momentos no caso em que $u_{i} \stackrel{\text { iid }}{\sim} N\left(0, \sigma_{u}^{2}\right)$. Cheng e Van Ness (1991) mostram a consistência e normalidade assintótica destes estimadores e obtêm de forma explícita a matriz de covariâncias assintótica, usando o método delta, dada a expressão explícita dos estimadores $\left(\hat{\alpha}, \widehat{\beta}, \hat{\sigma}_{\varepsilon}^{2}\right)$. No caso do modelo estrutural, os estimadores dados em (4.16) correspondem aos EMV, que coincidem com os estimadores obtidos pelo método dos momentos.

Supondo $E\left[u_{i}^{4}\right]<\infty$, a matriz de covariâncias assintótica dos estimadores em (4.16), para o modelo funcional, é dada por $n^{-1} \Omega_{n} \operatorname{com} \Omega_{n}$ como em (4.10), com

$$
\begin{aligned}
& \Omega_{1}=F \beta_{0}^{2}+G \sigma_{\varepsilon_{0}}^{2}, \\
& \Omega_{2}=H \beta_{0}^{3}+K \sigma_{\varepsilon_{0}}^{2} \beta_{0}, \\
& \Omega_{3}=\beta_{0}^{4}\left(\mu_{4}-\sigma_{u}^{4}\right)+2 \sigma_{\varepsilon_{0}}^{4}+4 \sigma_{u}^{2} \sigma_{\varepsilon_{0}}^{2} \beta_{0}^{2},
\end{aligned}
$$


com $F, G, H$ e $K$ como em (4.12), (4.13), (4.14) e (4.15), respectivamente. Depois de alguma álgebra, podemos escrever

$$
\Omega_{n}=\left(\begin{array}{ccc}
\delta+\bar{z}^{2} \psi-2 \bar{z} \gamma & -\bar{z} \psi+\gamma & -\bar{z} \rho-\beta S_{z z} \gamma \\
-\bar{z} \psi+\gamma & \psi & \rho \\
-\bar{z} \rho-\beta S_{z z} \gamma & \rho & \beta_{0}^{2} \tau+2 \delta^{2}
\end{array}\right)
$$

onde

$$
\begin{aligned}
\tau & =\beta_{0}\left(\mu_{4}-3 \sigma_{u}^{4}\right), \\
\delta & =\beta_{0}^{2} \sigma_{u}^{2}+\sigma_{\varepsilon_{0}}^{2} \\
\psi & =\frac{1}{S_{z z}^{2}}\left[\left(S_{z z}+\sigma_{u}^{2}\right) \delta+\beta_{0}^{2} \sigma_{u}^{4}+\tau\right] \\
\gamma & =\frac{\beta_{0}^{2}}{S_{z z}} \mu_{3} \\
\mathrm{e} \quad & =-\frac{\beta_{0}}{S_{z z}}\left(2 \sigma_{u}^{2} \delta+\tau\right) .
\end{aligned}
$$

No caso particular em que $u_{i} \stackrel{\text { iid }}{\sim} N\left(0, \sigma_{u}^{2}\right)$, segue que $\tau=\gamma=0, \delta=\beta_{0}^{2} \sigma_{u}^{2}+\sigma_{\varepsilon_{0}}^{2}, \psi=$ $\frac{1}{S_{z z}^{2}}\left[\left(S_{z z}^{(2)}+\sigma_{u}^{2}\right) \delta+\beta_{0}^{2} \sigma_{u}^{4}\right]$ e $\rho=-\frac{2 \beta_{0} \sigma_{u}^{2} \delta}{S_{z z}}$.

Sob a suposição $\bar{z} \rightarrow \mu<\infty$ e $S_{z z} \rightarrow \nu^{2}<\infty$, quando $n \rightarrow \infty, \Omega_{n}$ em (4.18) converge para a matriz

$$
\Omega=\left(\begin{array}{ccc}
\delta+\mu^{2} \psi & -\mu \psi & -\mu \rho \\
-\mu \psi & \psi & \rho \\
-\mu \rho & \rho & 2 \delta^{2}
\end{array}\right)
$$

$\operatorname{com} \delta$ como em (4.19), $\psi=\frac{1}{\nu^{4}}\left[\left(\nu^{4}+\sigma_{u}^{2}\right) \delta+\beta_{0}^{2} \sigma_{u}^{4}\right]$ e $\rho=-\frac{2 \beta_{0} \sigma_{u}^{2} \delta}{\nu^{2}}$.

Verificamos então que a matriz dada em (4.20) coincide com a matriz de covariâncias assintótica obtida por Cheng e Van Ness (1991), para os estimadores de momentos no modelo de regressão linear simples, normal.

A matriz em (4.20) também corresponde à matriz de covariâncias assintótica dos estimadores dados em (4.16) para o modelo de regressão linear simples, estrutural, normal, onde $z_{i} \stackrel{\text { iid }}{\sim} N\left(\mu, \nu^{2}\right)$. 


\subsection{O caso $\lambda_{k}=\sigma_{k}^{2} / \sigma_{u}^{2}, k=1, \ldots, p$, conhecidas}

Considere o modelo funcional

$$
\begin{aligned}
y_{i} & =\alpha+\beta z_{i}+\varepsilon_{i}, \\
x_{i} & =z_{i}+u_{i}, \quad i=1, \ldots, n,
\end{aligned}
$$

onde $\varepsilon_{i} \stackrel{\text { iid }}{\sim} N(0, \Sigma), \Sigma=\operatorname{Diag}\left(\sigma_{1}^{2}, \ldots, \sigma_{p}^{2}\right), u_{i} \stackrel{\text { iid }}{\sim}\left(0, \sigma_{u}^{2}\right)$ e as razões de variâncias $\lambda_{k}=$ $\sigma_{k}^{2} / \sigma_{u}^{2}, k=1, \ldots, p$, são conhecidas.

\subsubsection{O estimador do escore corrigido, propriedades assintóticas}

Sem perda de generalidade, podemos supor $\lambda_{k}=1, k=1, \ldots, p$, ou seja, $\sigma_{k}^{2}=\sigma_{u}^{2}=\phi$, $k=1, \ldots, p$, de modo que $\Sigma=\phi I_{p}$, onde $I_{p}$ denota a matriz identidade $p \times p$. Os $2 p+1$ parâmetros estruturais do modelo são dados por $\theta=\left(\alpha^{\prime}, \beta^{\prime}, \phi\right)^{\prime}$. O termo da log-verossimilhança "não observada", correspondente ao $i$-ésimo indivíduo é dado por

$$
\ell_{i}\left(\theta ; z_{i}, y_{i}\right)=-\frac{p}{2} \log (2 \pi)-\frac{p}{2} \log \phi-\frac{1}{2 \phi}\left(y_{i}-\alpha-\beta z_{i}\right)^{\prime}\left(y_{i}-\alpha-\beta z_{i}\right),
$$

$i=1, \ldots, n$. De modo que $U_{i}(\theta)=\left(U_{i \alpha}(\theta)^{\prime}, U_{i \beta}(\theta)^{\prime}, U_{i \phi}(\theta)\right)^{\prime}$ com

$$
\begin{aligned}
U_{i \alpha}(\theta) & =\frac{\partial \ell_{i}(\theta)}{\partial \alpha}=\frac{1}{\phi}\left(y_{i}-\alpha-\beta z_{i}\right), \\
U_{i \beta}(\theta) & =\frac{\partial \ell_{i}(\theta)}{\partial \beta}=\frac{1}{\phi}\left(y_{i}-\alpha-\beta z_{i}\right) z_{i}, \\
U_{i \phi}(\theta) & =\frac{\partial \ell_{i}(\theta)}{\partial \phi}=-\frac{p}{2 \phi}+\frac{1}{2 \phi^{2}}\left(y_{i}-\alpha-\beta z_{i}\right)^{\prime}\left(y_{i}-\alpha-\beta z_{i}\right), i=1, \ldots, n .
\end{aligned}
$$

Considerando $U_{i}^{*}(\theta)=\left(U_{i \alpha}^{*}(\theta)^{\prime}, U_{i \beta}^{*}(\theta)^{\prime}, U_{i \phi}^{*}(\theta)\right)^{\prime}$ com

$$
\begin{aligned}
U_{i \alpha}^{*}(\theta) & =\frac{1}{\phi}\left(y_{i}-\alpha-\beta x_{i}\right), \\
U_{i \beta}^{*}(\theta) & =\frac{1}{\phi}\left(y_{i}-\alpha-\beta x_{i}\right) x_{i}+\beta, \\
U_{i \phi}^{*}(\theta) & =\frac{1}{2 \phi^{2}}\left(y_{i}-\alpha-\beta x_{i}\right)^{\prime}\left(y_{i}-\alpha-\beta x_{i}\right)-\frac{1}{2 \phi}\left(\beta^{\prime} \beta+p\right), i=1, \ldots, n,
\end{aligned}
$$


segue que

$$
E\left[U_{i}^{*}\left(\theta ; x_{i}, y_{i}\right) \mid z_{i}, y_{i}\right]=U_{i}\left(\theta ; z_{i}, y_{i}\right), i=1, \ldots, n
$$

e portanto, $U^{*}(\theta ; X, Y)=\sum_{i=1}^{n} U_{i}^{*}\left(\theta ; x_{i}, y_{i}\right)$ é um escore corrigido. Neste caso, a logverossimilhança corrigida, ou seja, $\ell^{*}(\theta ; X, Y)$ tal que $E\left[\ell^{*}(\theta ; X, Y) \mid Z, Y\right]=\ell(\theta ; Z, Y)$ é dada por $\ell^{*}(\theta ; X, Y)=\sum_{i=1}^{n} \ell_{i}^{*}\left(\theta ; x_{i}, y_{i}\right)$, onde

$$
\ell_{i}^{*}\left(\theta ; x_{i}, y_{i}\right)=-\frac{p}{2} \log (2 \pi)-\frac{p}{2} \log \phi-\frac{1}{2 \phi}\left(y_{i}-\alpha-\beta x_{i}\right)^{\prime}\left(y_{i}-\alpha-\beta x_{i}\right)+\frac{1}{2} \beta^{\prime} \beta .
$$

Note que, agora,

$$
\frac{\partial \ell_{i}^{*}(\theta)}{\partial \phi}=-\frac{p}{2 \phi}+\frac{1}{2 \phi^{2}}\left(y_{i}-\alpha-\beta x_{i}\right)^{\prime}\left(y_{i}-\alpha-\beta x_{i}\right) \neq U_{i \phi}^{*}(\theta)
$$

Portanto, o escore corrigido $U^{*}(\theta ; X, Y)$ não corresponde ao gradiente da log-verossimilhança corrigida.

Resolvendo a equação $U^{*}(\theta ; X, Y)=0$, obtemos os estimadores $\hat{\theta}=\left(\hat{\alpha}^{\prime}, \hat{\beta}^{\prime}, \widehat{\phi}\right)^{\prime}$ como solução do seguinte sistema de equações:

$$
\begin{aligned}
& \alpha=\bar{y}-\beta \bar{x}, \\
& S_{x y}-\beta S_{x x}+\beta \phi=0, \\
& \phi=\frac{1}{\left(\beta^{\prime} \beta+p\right)} \sum_{k=1}^{p}\left(S_{y_{k} y_{k}}+\beta_{k}^{2} S_{x x}-2 \beta_{k} S_{x y_{k}}\right) .
\end{aligned}
$$

Depois de alguma álgebra, segue que $\widehat{\beta}=\left(\widehat{\beta}_{1}, \ldots, \widehat{\beta}_{p}\right)^{\prime}$ é solução do sistema de $p$ equações dado por

$$
\sum_{k=1}^{p}\left\{S_{x y_{j}} \beta_{k}-2 S_{x y_{k}} \beta_{k}^{2} \beta_{j}+\left(S_{y_{k} y_{k}}-S_{x x}\right) \beta_{j}+S_{x y_{j}}\right\}=0, j=1, \ldots, p
$$

A solução do sistema de equações (4.22) - (4.24) pode ser obtida usando um método iterativo.

Seja $\widehat{\beta}^{(m)}=\left(\widehat{\beta}_{1}^{(m)}, \ldots, \widehat{\beta}_{p}^{(m)}\right)^{\prime}$ solução de $(4.23)$ na $m$-ésima iteração, na iteração $m+1$ o algoritmo procede como segue: 
Etapa 1: Calcule $\widehat{\phi}^{(m+1)}=\phi\left(\widehat{\beta}^{(m)}\right)$, segundo a expressão (4.24).

Etapa 2: Calcule $\widehat{\beta}_{k}^{(m+1)}, k=1, \ldots, p$, como solução de

$$
S_{x y_{k}}-\beta_{k} S_{x x}+\beta_{k} \widehat{\phi}^{(m+1)}=0,
$$

ou seja,

$$
\widehat{\beta}_{k}^{(m+1)}=\frac{S_{x y_{k}}}{S_{x x}-\widehat{\phi}^{(m+1)}}, k=1, \ldots, p .
$$

A estimativa de $\alpha$, que não precisa do processo iterativo, é obtida a partir de (4.22).

O sistema (4.22) - (4.24), ou equivalentemente (4.25), tem raízes múltiplas, portanto a escolha da raiz apropriada deve ser feita cuidadosamente, pois podem existir raízes que não são consistentes. Se as equações (4.22) - (4.24) tivessem sido obtidas a partir do gradiente da log-verossimilhança corrigida, como na Seção 4.2, a raiz apropriada teria sido aquela que maximiza a log-verossimilhança corrigida. Duas possíveis soluções para o problema de raízes múltiplas são propostas em Stefanski e Carroll (1987) e Stefanski (1989). A primeira recomenda escolher a raiz mais próxima do estimador "naive" e com o mesmo sinal que este. Uma segunda solução é resolver o sistema (4.22) - (4.24) iterativamente, tomando como valor inicial o estimador "naive". Na prática, em duas ou três iterações é obtida a raiz apropriada.

Para $p=2$, é possível obter soluções explícitas para o sistema de $p$ equações (4.25). Neste caso, o sistema tem quatro raízes reais dadas por

$$
\begin{aligned}
& \hat{\beta}_{1(1,2)}=\frac{S_{x y_{1}}}{2}\left\{A \pm\left[\frac{8}{S_{x y_{1}}^{2}+S_{x y_{2}}^{2}}+A^{2}\right]^{1 / 2}\right\}, \\
& \hat{\beta}_{2(1,2)}=\frac{S_{x y_{2}}}{2}\left\{A \pm\left[\frac{8}{S_{x y_{1}}^{2}+S_{x y_{2}}^{2}}+A^{2}\right]^{1 / 2}\right\},
\end{aligned}
$$

onde

$$
A=\frac{S_{y_{1} y_{1}}+S_{y_{2} y_{2}}-2 S_{x x}}{S_{x y_{1}}^{2}+S_{x y_{2}}^{2}}
$$


Como o estimador "naive" para $\beta_{k}$ é dado por $\widehat{\beta}_{k}=\frac{S_{x y_{k}}}{S_{x x}}, k=1,2$, a raiz mais próxima do estimador "naive" é obtida considerando

$$
\begin{aligned}
& \widehat{\beta}_{1}=\frac{S_{x y_{1}}}{2}\left\{A+\left[\frac{8}{S_{x y_{1}}^{2}+S_{x y_{2}}^{2}}+A^{2}\right]^{1 / 2}\right\}, \\
& \hat{\beta}_{2}=\frac{S_{x y_{2}}}{2}\left\{A+\left[\frac{8}{S_{x y_{1}}^{2}+S_{x y_{2}}^{2}}+A^{2}\right]^{1 / 2}\right\} .
\end{aligned}
$$

A seguir encontramos a matriz de covariâncias assintótica de $\hat{\theta}$, para $p \geq 1$.

Depois de alguma álgebra, as matrizes $\bar{\Lambda}_{n}(\theta)$ e $\bar{\Gamma}_{n}(\theta)$ podem ser escritas como

$$
\bar{\Gamma}_{n}(\theta)=\left(\begin{array}{cc}
\frac{1}{\phi}\left[(A+B) \otimes \beta \beta^{\prime}+(A+C) \otimes I_{p}\right. & \frac{1}{\phi}\left(\begin{array}{cc}
A \otimes I_{p} & (0,-1)^{\prime} \otimes \beta \\
0 & \frac{\beta^{\prime} \beta+p}{2 \phi}
\end{array}\right), \\
-\frac{1}{\phi}\left(D^{\prime} \otimes \beta^{\prime}\right) & \frac{1}{\phi}(D \otimes \beta)
\end{array}\right),
$$

onde

$$
\begin{aligned}
A & =\left(\begin{array}{cc}
1 & \bar{z} \\
\bar{z} & S_{z z}+\bar{z}^{2}
\end{array}\right) \\
B & =\frac{1}{\phi}\left(\begin{array}{cc}
0 & \mu_{3} \\
\mu_{3} & 2 \bar{z} \mu_{3}+\mu_{4}-\phi^{2}
\end{array}\right) \\
C & =\left(\begin{array}{ll}
0 & 0 \\
0 & \phi
\end{array}\right) \quad \text { e } \quad D=-\frac{1}{2 \phi^{2}}\left(\begin{array}{c}
\mu_{3} \beta^{\prime} \beta \\
\left(\bar{z} \mu_{3}+\mu_{4}-\phi^{2}\right) \beta^{\prime} \beta+2 \phi^{2}
\end{array}\right) .
\end{aligned}
$$

Se a seqüência de parâmetros incidentais verifica (4.7) e (4.8), segue dos Teoremas 2.1 e 2.2 que o estimador $\hat{\theta}=\left(\widehat{\alpha}^{\prime}, \widehat{\beta}^{\prime}, \widehat{\phi}\right)^{\prime}$, definido como solução do sistema $(4.22)-(4.24)$, é consistente e assintoticamente normal, com vetor de médias $\theta_{0}=\left(\alpha_{0}^{\prime}, \beta_{0}^{\prime}, \phi_{0}\right)^{\prime}$ e matriz de covariâncias dada por $n^{-1} \Omega_{n}$,

$$
\Omega_{n}=\bar{\Lambda}_{n}^{-1} \bar{\Gamma}_{n} \bar{\Lambda}_{n}^{-1}=\left(\begin{array}{ll}
\Omega_{1} & \Omega_{2} \\
\Omega_{2}^{\prime} & \Omega_{3}
\end{array}\right),
$$

onde

$$
\begin{aligned}
& \Omega_{1}=F \otimes\left(\beta_{0} \beta_{0}^{\prime}\right)+G \otimes I_{p}, \\
& \Omega_{2}=H \otimes \beta_{0} \\
& \Omega_{3}=\frac{1}{\left(\beta_{0}^{\prime} \beta_{0}+p\right)^{2}}\left[\left(\mu_{4}-\phi_{0}^{2}\right)\left(\beta_{0}^{\prime} \beta_{0}\right)^{2}+4 \phi_{0}^{2}\left(\beta_{0}^{\prime} \beta_{0}\right)+2 p \phi_{0}^{2}\right],
\end{aligned}
$$


com

$$
\begin{aligned}
F & =\frac{1}{S_{z z}^{2} a^{2}}\left(\begin{array}{cc}
S_{z z} \phi\left(S_{z z}+\bar{z}^{2}\right) a^{2}-2 p S_{z z} \bar{z} \mu_{3} a+\bar{z}^{2} b & -S_{z z} \bar{z} \phi a^{2}+p S_{z z} \mu_{3} a-\bar{z} b \\
-S_{z z} \bar{z} \phi a^{2}+p S_{z z} \mu_{3} a-\bar{z} b & S_{z z} \phi a^{2}+b
\end{array}\right) \\
G & =\frac{\phi}{S_{z z}^{2}}\left(\begin{array}{cc}
S_{z z}^{2}+\bar{z}^{2}\left(S_{z z}+\phi\right) & -\bar{z}\left(S_{z z}+\phi\right) \\
-\bar{z}\left(S_{z z}+\phi\right) & S_{z z}+\phi
\end{array}\right) \\
H & =\frac{p-a}{S_{z z} a^{2}}\left(\begin{array}{c}
S_{z z} \mu_{3} a-\frac{\bar{z} b}{p} \\
\frac{b}{p}
\end{array}\right)
\end{aligned}
$$

onde $a=\beta^{\prime} \beta+p$ e $b=p^{2} \mu_{4}-\left(p^{2}+2 p\right) \phi^{2}$.

No caso do modelo estrutural com $z_{i} \stackrel{\text { iid }}{\sim} N\left(\mu, \nu^{2}\right)$ a matriz de covariâncias assintótica é dada por $n^{-1} \Omega \operatorname{com} \Omega$ como $\Omega_{n}$ em (4.28), $\operatorname{com} \mu$ e $\nu^{2}$ no lugar de $\bar{z}$ e $S_{z z}$, respectivamente, em $(4.30)-(4.32)$.

Observação: Não há perda de generalidade em considerar $\lambda_{k}=1$, ou equivalentemente, $\sigma_{k}^{2}=\sigma_{u}^{2}=\phi, k=1, \ldots, p$. Com efeito, se no modelo (4.21) assumirmos $\varepsilon_{i} \stackrel{\text { iid }}{\sim} N(0, \Sigma)$, com $\Sigma=\operatorname{Diag}\left(\sigma_{1}^{2}, \ldots, \sigma_{p}^{2}\right)=\phi \Sigma_{0}$, onde $\Sigma_{0}=\operatorname{Diag}\left(\lambda_{1}, \ldots, \lambda_{p}\right), \lambda_{k}$ conhecidas, $k=1, \ldots, p$, e $u_{i} \stackrel{\text { iid }}{\sim}(0, \phi)$, e considerarmos os dados transformados $y_{i}^{*}=\Sigma_{0}^{-1 / 2} y_{i}$, o novo modelo

$$
\begin{aligned}
& y_{i}^{*}=\alpha^{*}+\beta^{*} z_{i}+\varepsilon_{i}^{*} \\
& x_{i}=z_{i}+u_{i},
\end{aligned}
$$

$\operatorname{com} \alpha^{*}=\Sigma_{0}^{-1 / 2} \alpha, \beta^{*}=\Sigma_{0}^{-1 / 2} \beta$ e $\varepsilon_{i}^{*}=\Sigma_{0}^{-1 / 2} \varepsilon_{i}$ é tal que $\varepsilon_{i}^{*} \stackrel{\text { iid }}{\sim} N\left(0, \Sigma^{*}\right)$ com $\Sigma^{*}=$ $\phi I_{p}$. Assim, os estimadores para $\alpha^{*}$ e $\beta^{*}$ baseados nos dados transformados podem ser convertidos em estimadores para $\alpha$ e $\beta$.

\subsubsection{O modelo de regressão linear simples}

Quando $p=1$, o modelo (4.21) corresponde ao modelo de regressão linear simples, com razão de variâncias conhecida. 
Os três parâmetros estruturais do modelo são dados por $\theta=(\alpha, \beta, \phi)^{\prime}$. As equações $(4.22)-(4.24)$ se reduzem a

$$
\begin{aligned}
& \alpha=\bar{y}-\beta \bar{x}, \\
& S_{x y}-\beta S_{x x}+\beta \phi=0, \\
& \phi=\frac{1}{\beta^{2}+1}\left(S_{y y}+\beta^{2} S_{x x}-2 \beta S_{x y}\right),
\end{aligned}
$$

de modo que

$$
\hat{\beta}_{(1,2)}=\frac{S_{y y}-S_{x x} \pm \sqrt{\left(S_{y y}-S_{x x}\right)^{2}+4 S_{x y}^{2}}}{2 S_{x y}}, \quad S_{x y} \neq 0 .
$$

A raiz apropriada é obtida como aquela mais próxima do estimador "naive" que, neste caso, corresponde a

$$
\widehat{\beta}=\frac{S_{y y}-S_{x x}+\sqrt{\left(S_{y y}-S_{x x}\right)^{2}+4 S_{x y}^{2}}}{2 S_{x y}},
$$

$\widehat{\alpha}$ e $\hat{\phi}$ são obtidos substituindo (4.36) em (4.33) e (4.35), respectivamente.

$\widehat{\alpha}$ e $\widehat{\beta}$ coincidem com os EMV para $\alpha$ e $\beta$, respectivamente, no modelo funcional, sob normalidade de $u_{i}, \widehat{\phi}$ sendo consistente é diferente do EMV, o qual resulta inconsistente (Lindley, 1947). $\widehat{\phi}$ corresponde ao estimador de momentos no modelo funcional. No caso do modelo estrutural, os estimadores $\widehat{\alpha}, \widehat{\beta}$ e $\widehat{\phi}$ coincidem com os EMV e os estimadores de momentos (veja Cheng e Van Ness, 1994).

A matriz de covariâncias assintótica de $(\widehat{\alpha}, \widehat{\beta}, \widehat{\phi})^{\prime}$ no modelo funcional é dada por $n^{-1} \Omega_{n}$, com $\Omega_{n}$ como em (4.28), onde

$$
\begin{aligned}
& \Omega_{1}=F \beta_{0}^{2}+G \\
& \Omega_{2}=H \beta_{0}, \\
& \Omega_{3}=\frac{1}{\left(\beta_{0}^{2}+1\right)^{2}}\left[\left(\mu_{4}-\phi^{2}\right) \beta_{0}^{4}+4 \phi^{2} \beta_{0}^{2}+2 \phi_{0}^{2}\right],
\end{aligned}
$$

com F, G e $H$ como em (4.30), (4.31) e (4.32), respectivamente. Depois de alguma álgebra, 
$\Omega_{n}$ pode ser escrita como

$$
\Omega_{n}=\left(\begin{array}{ccc}
\delta+\bar{z}^{2} \psi-2 \bar{z} \gamma & -\bar{z} \psi+\gamma & -\bar{z} \rho-\frac{\beta_{0}^{3}}{\beta_{0}^{2}+1} \mu_{3} \\
-\bar{z} \psi+\gamma & \psi & \rho \\
-\bar{z} \rho-\frac{\beta_{0}^{3}}{\beta_{0}^{2}+1} \mu_{3} & \rho & \frac{\beta^{4} \tau+2 \delta^{2}}{\left(\beta_{0}^{2}+1\right)^{2}}
\end{array}\right),
$$

onde

$$
\begin{aligned}
\tau & =\mu_{4}-3 \phi_{0}^{2}, \\
\delta & =\phi_{0}\left(\beta_{0}^{2}+1\right) \\
\psi & =\frac{\delta}{S_{z z}}+\frac{\beta_{0}^{2} \tau+\delta^{2}}{\left(\beta_{0}^{2}+1\right)^{2} S_{z z}^{2}}, \\
\gamma & =\frac{\beta_{0}^{2}}{\left(\beta_{0}^{2}+1\right) S_{z z}} \mu_{3}, \\
\rho & =\frac{-\beta_{0}^{3} \tau}{\left(\beta_{0}^{2}+1\right)^{2} S_{z z}} .
\end{aligned}
$$

No caso particular em que $u_{i} \stackrel{\text { iid }}{\sim} N\left(0, \sigma_{u}^{2}\right)$, temos que $\tau=\rho=\gamma=0, \delta=\phi_{0}\left(\beta_{0}^{2}+1\right)$ e $\psi=\frac{\phi_{0}\left(\beta_{0}^{2}+1\right)}{S_{z z}}+\frac{\phi_{0}^{2}}{S_{z z}^{2}}$, e a matriz $\Omega_{n}$ em (4.37) pode ser reescrita como

$$
\Omega_{n}=\left(\begin{array}{ccc}
\delta+\bar{z}^{2} \psi & -\bar{z} \psi & 0 \\
-\bar{z} \psi & \psi & 0 \\
0 & 0 & \frac{2 \delta^{2}}{\left(\beta_{0}^{2}+1\right)^{2}}
\end{array}\right)
$$

Esta matriz coincide com a correspondente matriz de covariâncias, dada em Mak (1982).

Sob a suposição que $\bar{z} \rightarrow \mu<\infty$ e $S_{z z} \rightarrow \nu^{2}<\infty$, quando $n \rightarrow \infty, \Omega_{n}$ em (4.39) converge para a matriz

$$
\Omega=\left(\begin{array}{ccc}
\delta+\mu^{2} \psi & -\mu \psi & 0 \\
-\mu \psi & \psi & 0 \\
0 & 0 & \frac{2 \delta^{2}}{\left(\beta_{0}^{2}+1\right)^{2}}
\end{array}\right),
$$

$\operatorname{com} \delta=\phi_{0}\left(\beta_{0}^{2}+1\right)$ e $\psi=\frac{\phi_{0}\left(\beta_{0}^{2}+1\right)}{\nu^{2}}+\frac{\phi_{0}^{2}}{\nu^{4}}$, coincidindo com a matriz obtida em Gleser (1985).

\subsubsection{Comparação de estimadores}

Nesta seção calculamos a eficiência relativa assintótica do estimador do escore corrigido do parâmetro $\beta$, com respeito a outros dois estimadores propostos por Galea-Rojas (1995), 
para o modelo (4.21) no caso $p=2$, com $u_{i}$ normalmente distribuído.

Galea-Rojas utiliza os resultados de Mak (1982) para encontrar os EMV de $\alpha, \beta$ e $\phi$ e sua matriz de covariâncias assintótica. É mostrado que os EMV de $\alpha, \beta$ e $\phi$, que denotaremos por $\tilde{\alpha}, \tilde{\beta}$ e $\tilde{\phi}$ satisfazem

$$
\begin{aligned}
\alpha & =\bar{y}-\beta \bar{x} \\
\beta & =\sum_{i=1}^{n}\left(y_{i}-\bar{y}\right) \widehat{z}_{i} / \sum_{i=1}^{n}\left(\widehat{z}_{i}-\bar{x}\right)^{2} \\
\phi & =\frac{1}{n(p+1)} \sum_{i=1}^{n}\left\{\left(x_{i}-\widehat{z}_{i}\right)^{2}+\left(y_{i}-\alpha-\beta \widehat{z}_{i}\right)^{\prime}\left(y_{i}-\alpha-\beta \widehat{z}_{i}\right)\right\}
\end{aligned}
$$

onde $\widehat{z}_{i}=\widehat{z}_{i}(\theta)=\frac{1}{c}\left[x_{i}+\left(y_{i}-\alpha\right)^{\prime} \beta\right]$ é o EMV de $z_{i}$ dado $\theta, \operatorname{com} c=\beta^{\prime} \beta+1$.

A solução de (4.40) - (4.42) é obtida usando um método iterativo. $\tilde{\phi}$ não é consistente, mas $\frac{p+1}{p} \tilde{\phi}$ o é. Assim, $\left(\dot{\tilde{\alpha}}^{\prime}, \widetilde{\beta}^{\prime}, \frac{(p+1)}{2} \widetilde{\phi}\right)^{\prime}$ é consistente e assintoticamente normal com vetor de médias $\theta_{0}=\left(\alpha_{0}^{\prime}, \beta_{0}^{\prime}, \phi_{0}\right)^{\prime}$ e matriz de covariâncias assintótica $n^{-1} \Omega_{n}$, onde

$$
\Omega_{n}=\frac{\phi_{0}}{c_{0}}\left(\begin{array}{cc}
K \otimes B_{0}^{-1} & 0 \\
0 & \frac{2 \phi_{0}^{2}}{c_{0} p}
\end{array}\right), \quad c_{0}=\beta_{0}^{\prime} \beta_{0}+1,
$$

com

$$
\begin{aligned}
& K=\frac{1}{S_{z z}}\left(\begin{array}{cc}
S_{z z}+\bar{z}^{2} d_{0} & -\bar{z} d_{0} \\
-\bar{z} d_{0} & d_{0}
\end{array}\right), d_{0}=1+\frac{\phi_{0}}{c_{0} S_{z z}}, \\
& B_{0}^{-1}=\frac{1}{c_{0}}\left[I_{p}+\beta_{0} \beta_{0}^{\prime}\right] .
\end{aligned}
$$

Estimadores de momentos para o parâmetro $\beta$ também são propostos em Galea-Rojas (1995), e suas propriedades assintóticas obtidas para o caso $p=2$.

Os estimadores de momentos de $\beta_{1}$ e $\beta_{2}$ são dados por

$$
\beta_{1}^{*}=\frac{S_{y_{1} y_{2}}}{S_{x y_{2}}} \quad \text { e } \quad \beta_{2}^{*}=\frac{S_{y_{1} y_{2}}}{S_{x y_{1}}} .
$$

É mostrado que

$$
\sqrt{n}\left(\beta_{1}^{*}-\beta_{1}\right) \stackrel{\mathcal{D}}{\longrightarrow} N\left(0, \sigma_{\beta_{1}^{*}}^{2}\right)
$$


com

$$
\sigma_{\beta_{i}^{*}}^{2}=\frac{\phi\left(1+\beta_{1}^{2}\right)}{\nu^{2}}\left(1+\frac{\phi}{\nu^{2} \beta_{2}^{2}}\right)
$$

e

$$
\sqrt{n}\left(\beta_{2}^{*}-\beta_{2}\right) \stackrel{\mathcal{D}}{\longrightarrow} N\left(0, \sigma_{\beta_{2}^{*}}^{2}\right)
$$

com

$$
\sigma_{\beta_{2}^{*}}^{2}=\frac{\phi\left(1+\beta_{2}^{2}\right)}{\nu^{2}}\left(1+\frac{\phi}{\nu^{2} \beta_{1}^{2}}\right),
$$

onde é assumido que $S_{z z} \rightarrow \nu^{2}<\infty$, quando $n \rightarrow \infty$.

Para $p=2$ e $u_{i} \stackrel{\text { iid }}{\sim} N(0, \phi)$ a matriz de covariâncias assintótica do estimador do escore corrigido $\hat{\theta}=\left(\widehat{\alpha}^{\prime}, \widehat{\beta}^{\prime}, \widehat{\phi}\right)^{\prime}$ é dada pela expressão (4.28) com $\mu_{3}=0, \mu_{4}=3 \phi^{2}, a=\beta_{1}^{2}+\beta_{2}^{2}+2$ e $b=4 \phi^{2}$ nas expressões $(4.30)-(4.32)$.

Portanto, assumindo que $S_{z z} \rightarrow \nu^{2}<\infty$, quando $n \rightarrow \infty$, de (4.28) segue que

$$
\sqrt{n}\left(\widehat{\beta}_{1}-\beta_{1}\right) \stackrel{\mathcal{D}}{\longrightarrow} N\left(0, \sigma_{\widehat{\beta}_{1}}^{2}\right)
$$

com

$$
\sigma_{\widehat{\beta}_{1}}^{2}=\frac{\phi}{\nu^{2}}\left(1+\beta_{1}^{2}\right)+\frac{\phi^{2}}{\nu^{4}}\left(1+\frac{4 \beta_{1}^{2}}{a^{2}}\right)
$$

e de (4.43) temos que

$$
\sqrt{n}\left(\widetilde{\beta}_{1}-\beta_{1}\right) \stackrel{\mathcal{D}}{\longrightarrow} N\left(0, \sigma_{\widetilde{\beta}_{1}}^{2}\right)
$$

com

$$
\sigma_{\widetilde{\beta}_{1}}^{2}=\frac{\phi}{\nu^{2}}\left(1+\beta_{1}^{2}\right)+\frac{\phi^{2}}{\nu^{4}} \frac{\left(1+\beta_{1}^{2}\right)}{c}
$$

onde $c=\beta_{1}^{2}+\beta_{2}^{2}+1=a-1$.

De (4.45) e (4.46) temos que a eficiência relativa assintótica do EMV $\widetilde{\beta}_{1}$ com respeito ao estimador do escore corrigido $\widehat{\beta}_{1}$ é dada por

$$
\operatorname{ARE}\left(\widetilde{\beta}_{1} \mid \widehat{\beta}_{1}\right)=\frac{\frac{\phi}{\nu^{2}}\left(1+\beta_{1}^{2}\right)+\frac{\phi^{2}}{\nu^{4}}\left(1+4 \frac{\beta_{1}^{2}}{a^{2}}\right)}{\frac{\phi}{\nu^{2}}\left(1+\beta_{1}^{2}\right)+\frac{\phi^{2}}{\nu^{4}}\left(\frac{1+\beta_{1}^{2}}{c}\right)}>1,
$$


pois $1+\frac{4 \beta_{1}^{2}}{a^{2}}>\frac{1+\beta_{1}^{2}}{c}$. Ou seja, o EMV $\widetilde{\beta}_{1}$ é mais eficiente que o estimador do escore corrigido $\widehat{\beta}_{1}$.

Por outro lado, de (4.44) e (4.45), temos que a eficiência relativa assintótica do estimador do escore corrigido $\widehat{\beta}_{1}$, com respeito ao estimador de momentos $\beta_{1}^{*}$ é dada por

$$
\operatorname{ARE}\left(\widehat{\beta}_{1} \mid \beta_{1}^{*}\right)=\frac{\frac{\phi}{\nu^{2}}\left(1+\beta_{1}^{2}\right)+\frac{\phi^{2}}{\nu^{4}}\left(\frac{1+\beta_{1}^{2}}{\beta_{2}^{2}}\right)}{\frac{\phi}{\nu^{2}}\left(1+\beta_{1}^{2}\right)+\frac{\phi^{2}}{\nu^{4}}\left(1+\frac{4 \beta_{1}^{2}}{a^{2}}\right)} .
$$

Note que, quando $\frac{1+\beta_{1}^{2}}{\beta_{2}^{2}}>1+\frac{4 \beta_{1}^{2}}{a^{2}}$, teremos $\operatorname{ARE}\left(\widehat{\beta}_{1} \mid \beta_{1}^{*}\right)>1 \mathrm{e}$, conseqüentemente, nesse caso o estimador do escore corrigido $\widehat{\beta}_{1}$ será mais eficiente do que o estimador de momentos $\beta_{1}^{*}$.

\subsubsection{Testes de hipóteses}

Usando os resultados da Seção 3.2, podemos implementar testes assintóticos para provar hipóteses de interesse. Particularmente, interessa testar se os instrumentos medem sem vício a característica de interesse $z$, ou seja, a hipótese

$$
H_{0}:\left(\begin{array}{c}
\alpha \\
\beta
\end{array}\right)=\left(\begin{array}{c}
0 \\
1_{p}
\end{array}\right)
$$

Para testar $H_{0}$, consideremos as estatísticas de Wald $W_{c}$ e escore $Q_{c}$, baseadas no estimador do escore corrigido, definidas em (3.5) e (3.6), respectivamente, com distribuição assintótica $\chi_{2 p}^{2}$.

Na notação da Seção $3.2, \psi=\left(\alpha^{\prime}, \beta^{\prime}\right)^{\prime}$ é o parâmetro de interesse e $\lambda=\phi$ é o parâmetro "nuisance". $\hat{\theta}=\left(\widehat{\alpha}^{\prime}, \widehat{\beta}^{\prime}, \widehat{\phi}\right)^{\prime}$ é o estimador do escore corrigido irrestrito, i.e., solução do sistema $(4.22)-(4.24)$ e $\widehat{\theta}_{0}=\left(0^{\prime}, 1_{p}^{\prime}, \widehat{\phi}_{0}\right)^{\prime}$ é o estimador restrito a $H_{0}$, onde

$$
\widehat{\phi}_{0}=\frac{1}{2 p} \sum_{k=1}^{p}\left(S_{y_{k} y_{k}}+S_{x x}-2 S_{x y_{k}}\right) \text {. }
$$

Assumimos que $u_{i} \stackrel{\text { iid }}{\sim} N(0, \phi)$ e que as matrizes $\bar{\Lambda}_{n}(\theta)$ e $\bar{\Gamma}_{n}(\theta)$ convergem para matrizes $\Lambda(\theta)$ e $\Gamma(\theta)$, definidas positivas. Esta suposição equivale a assumir que

$$
\bar{z} \underset{n \rightarrow \infty}{ } \mu<\infty
$$




$$
S_{z z} \overline{n \rightarrow \infty} \nu^{2}<\infty
$$

no caso de um modelo funcional (para o modelo estrutural a lei forte dos grandes números garante estas propriedades do primeiro e segundo momentos amostrais da variável $z$ ).

A estatística de Wald $W_{c}$ pode ser escrita como

$$
W_{c}=n\left[\hat{\alpha}^{\prime},\left(\widehat{\beta}-1_{p}\right)^{\prime}\right] \widehat{\Omega}_{\psi \psi}^{-1}\left(\hat{\theta}_{0}\right)\left[\hat{\alpha}^{\prime},\left(\hat{\beta}-1_{p}\right)^{\prime}\right]^{\prime},
$$

onde $\widehat{\Omega}_{\psi \psi}$ é um estimador consistente da matriz $\Omega_{\psi \psi}=\left(\Lambda^{-1} \Gamma \Lambda^{-1}\right)_{\psi \psi}$ dada por

$$
\Omega_{\psi \psi}=F \otimes\left(\beta \beta^{\prime}\right)+G \otimes I_{p},
$$

onde

e

$$
F=\frac{1}{\nu^{4} a^{2}}\left(\begin{array}{cc}
\nu^{2} \phi\left(\nu^{2}+\mu^{2}\right) a^{2}+\mu^{2} b & -\nu^{2} \mu \phi a^{2}-\mu b \\
-\nu^{2} \mu \phi a^{2}-\mu b & \nu^{2} \phi a^{2}+b
\end{array}\right)
$$

$$
G=\frac{\phi}{\nu^{4}}\left(\begin{array}{cc}
\nu^{4}+\mu^{2}\left(\nu^{2}+\phi\right) & -\mu\left(\nu^{2}+\phi\right) \\
-\mu\left(\nu^{2}+\phi\right) & \nu^{2}+\phi
\end{array}\right),
$$

com $a=\beta^{\prime} \beta+p$ e $b=2 p(p-1) \phi^{2}$ (veja as expressões (4.29) - (4.31) da Seção 4.3.1).

Depois de alguma álgebra, temos que $\Omega_{\psi \psi}^{-1}$ pode ser escrita como

$$
\Omega_{\psi \psi}^{-1}(\theta)=\frac{1}{\phi}\left(\begin{array}{cc}
\left(I_{p}+\beta \beta^{\prime}\right)^{-1} & \mu\left(I_{p}+\beta \beta^{\prime}\right)^{-1} \\
\mu\left(I_{p}+\beta \beta^{\prime}\right)^{-1} & \mu^{2}\left(I_{p}+\beta \beta^{\prime}\right)^{-1}+\frac{\nu^{4}}{\nu^{2}+\phi}\left(I_{p}+r \beta \beta^{\prime}\right)^{-1}
\end{array}\right),
$$

onde

$$
r=\frac{1}{\nu^{2}+\phi}\left[\nu^{2}+\frac{2 p(p-1) \phi}{\left(\beta^{\prime} \beta+p\right)^{2}}\right] .
$$

Note que, para estimar $\Omega_{\psi \psi}$ consistentemente, basta estimar $\mu$ e $\nu^{2}$ consistentemente. Com efeito, da lei fraca dos grandes números e das suposições (4.47) e (4.48), temos que

$$
\bar{x} \stackrel{\mathrm{P}}{\longrightarrow} \mu \quad \text { e } \quad S_{x x}-\phi \stackrel{\mathrm{p}}{\longrightarrow} \nu^{2} \text {, quando } n \rightarrow \infty .
$$

Assim, $\widehat{\Omega}_{\psi \psi}^{-1}\left(\widehat{\theta}_{0}\right)$ é dado por

$$
\widehat{\Omega}_{\psi \psi}^{-1}\left(\widehat{\theta}_{0}\right)=\frac{1}{\widehat{\phi}_{0}}\left(\begin{array}{cc}
\left(I_{p}+1_{p} 1_{p}^{\prime}\right)^{-1} & \bar{x}\left(I_{p}+1_{p} 1_{p}^{\prime}\right)^{-1} \\
\bar{x}\left(I_{p}+1_{p} 1_{p}^{\prime}\right)^{-1} & \bar{x}^{2}\left(I_{p}+1_{p} 1_{p}^{\prime}\right)^{-1}+\frac{\left(S_{x x}-\phi\right)^{2}}{S_{x x}}\left(I_{p}+\hat{r} 1_{p} 1_{p}^{\prime}\right)^{-1}
\end{array}\right),
$$


onde

$$
\widehat{r}=1-\frac{(p+1) \widehat{\phi}_{0}}{2 p S_{x x}} .
$$

O estimador "sanduíche" de $\Omega$ proposto na Seção 2.3.4 também poderia ser utilizado para estimar $\Omega_{\psi \psi}$.

Finalmente, usando (4.51), a estatística $W_{c}$ pode ser escrita como

$$
\begin{aligned}
W_{c}= & \frac{n}{\widehat{\phi}_{0}}\left\{\hat{\alpha}^{\prime}\left(I_{p}+1_{p} 1_{p}^{\prime}\right)^{-1} \hat{\alpha}+2 \bar{x} \widehat{\alpha}^{\prime}\left(I_{p}+1_{p} 1_{p}^{\prime}\right)^{-1}\left(\widehat{\beta}-1_{p}\right)\right. \\
& \left.+\left(\widehat{\beta}-1_{p}\right)^{\prime}\left[\bar{x}^{2}\left(I_{p}+1_{p} 1_{p}^{\prime}\right)^{-1}+\frac{\left(S_{x x}-\widehat{\phi}_{0}\right)^{2}}{S_{x x}}\left(I_{p}+\widehat{r} 1_{p} 1_{p}^{\prime}\right)^{-1}\right]\left(\widehat{\beta}-1_{p}\right)\right\}
\end{aligned}
$$

Por outro lado, temos que a estatística do escore $Q_{c}$ é dada por

$$
Q_{c}=n^{-1} U_{\psi}^{*}\left(\hat{\theta}_{0}\right)^{\prime} \hat{\Lambda}_{\psi \psi . \phi}^{-1}\left(\hat{\theta}_{0}\right) \hat{\Omega}_{\psi \psi}^{-1}\left(\hat{\theta}_{0}\right) \hat{\Lambda}_{\psi \psi . \phi}^{-1} U_{\psi}^{*}\left(\hat{\theta}_{0}\right),
$$

onde o escore corrigido $U_{\psi}^{*}(\theta)$ obtido na Seção 2.3.1 é

$$
U_{\psi}^{*}(\theta)=\left(\begin{array}{l}
U_{\alpha}^{*}(\theta) \\
U_{\beta}^{*}(\theta)
\end{array}\right)=\frac{1}{\phi} \sum_{i=1}^{n}\left(\begin{array}{c}
y_{i}-\alpha-\beta x_{i} \\
\left(y_{i}-\alpha-\beta x_{i}\right) x_{i}+\beta \phi
\end{array}\right) .
$$

Assim,

$$
U_{\psi}^{*}\left(\widehat{\theta}_{0}\right)=\frac{n}{\hat{\phi}_{0}}\left(\begin{array}{c}
\bar{y}-1_{p} \bar{x} \\
S_{x y}+\bar{x} \bar{y}-1_{p}\left(S_{x x}-\phi+\bar{x}^{2}\right)
\end{array}\right) .
$$

Dado que

$$
\Lambda_{\psi \psi \cdot \phi}^{-1}=\phi_{0}\left(A^{-1} \otimes I_{p}\right)
$$

(veja Seção 4.3.1), onde

$$
A^{-1}=\frac{1}{\nu^{2}}\left(\begin{array}{cc}
\nu^{2}+\mu^{2} & -\mu \\
-\mu & 1
\end{array}\right),
$$

um estimador consistente de $\Lambda_{\psi \psi . \phi}^{-1}$ é

$$
\widehat{\Lambda}_{\psi \psi . \phi}^{-1}\left(\widehat{\theta}_{0}\right)=\widehat{\phi}_{0}\left(\hat{A}^{-1} \otimes I_{p}\right),
$$

com

$$
\widehat{A}^{-1}=\frac{1}{S_{x x}-\widehat{\phi}_{0}}\left(\begin{array}{cc}
S_{x x}-\widehat{\phi}_{0}+\bar{x}^{2} & -\bar{x} \\
-\bar{x} & 1
\end{array}\right) .
$$


De (4.51) e (4.53), temos então que

$$
=\widehat{\phi}_{0}\left(\begin{array}{cc}
\hat{\Lambda}_{\psi \psi \cdot \phi}^{-1}\left(\hat{\theta}_{0}\right) \widehat{\Omega}_{\psi \psi}^{-1}\left(\hat{\theta}_{0}\right) \Lambda_{\psi \psi, \phi}^{-1}\left(\hat{\theta}_{0}\right) \\
-\frac{\bar{x}}{S_{x x}}\left(I_{p}+\widehat{r} 1_{p} 1_{p}^{\prime}\right)^{-1} & \frac{1}{S_{x x}}\left(I_{p}+\widehat{r} 1_{p} 1_{p}^{\prime}\right)^{-1}+\frac{\bar{x}^{2}}{S_{x x}}\left(I_{p}+\widehat{r} 1_{p} 1_{p}^{\prime}\right)^{-1}
\end{array}\right) .
$$

Portanto, a estatística $Q_{c}$ pode ser escrita como

$$
\begin{aligned}
Q_{c}= & \frac{n}{\widehat{\phi}_{0}}\left\{\left(\bar{y}-1_{p} \bar{x}\right)^{\prime}\left[\left(I_{p}+1_{p} 1_{p}^{\prime}\right)^{-1}+\frac{\bar{x}^{2}}{S_{x x}}\left(I_{p}+\widehat{r} 1_{p} 1_{p}^{\prime}\right)^{-1}\right]\left(\bar{y}-1_{p} \bar{x}\right)\right. \\
& -\frac{2 \bar{x}}{S_{x x}}\left[S_{x y}+\bar{x} \bar{y}-1_{p}\left(S_{x x}-\widehat{\phi}_{0}+\bar{x}^{2}\right)\right]^{\prime}\left(I_{p}+\widehat{r} 1_{p} 1_{p}^{\prime}\right)^{-1}\left(\bar{y}-1_{p} \bar{x}\right) \\
& +\frac{1}{S_{x x}}\left[S_{x y}+\bar{x} \bar{y}-1_{p}\left(S_{x x}-\widehat{\phi}_{0}+\bar{x}^{2}\right)\right]^{\prime}\left(I_{p}+\widehat{r} 1_{p} 1_{p}^{\prime}\right)^{-1}\left[S_{x y}+\bar{x} \bar{y}\right. \\
& \left.\left.-1_{p}\left(S_{x x}-\widehat{\phi}_{0}+\bar{x}^{2}\right)\right]\right\} .
\end{aligned}
$$

\subsubsection{Ilustração numérica}

Consideremos os seguintes dados (Tabela 4.1), tomados de Jaech (1985), relativos a um experimento no qual a densidade de 43 partes cilíndricas de urânio sintetizado foi medida por vários métodos. Analisaremos os dados correspondentes a três dos métodos.

Assumimos o modelo (4.21), com $u_{i}$ normalmente distribuída, $n=43$ e $p=2$.

Temos que

$$
\begin{aligned}
& \bar{x}=4.397, \quad \bar{y}=(4.370,4.436)^{\prime} \\
& S_{x x}=0.04391, \quad S_{x y}=(0.03449,0.03933)^{\prime} \quad \mathrm{e} \\
& S_{y y}=\left(\begin{array}{ll}
0.04013 & 0.03483 \\
& 0.06442
\end{array}\right) .
\end{aligned}
$$

Usando as expressões (4.22), (4.24), (4.26) e (4.27), obtemos os seguintes estimadores do escore corrigido:

$$
\begin{aligned}
\hat{\alpha} & =(-0.21963,-0.79769)^{\prime} \\
\hat{\beta} & =(1.04381,1.19029)^{\prime} \\
\text { e } \quad \hat{\phi} & =0.01397 .
\end{aligned}
$$


Tabela 4.1: Medidas de densidade de urânio sintetizado, fornecidas por três métodos

\begin{tabular}{ccc|ccc}
\hline Método padrão & Método 1 & Método 2 & Método padrão & Método 1 & Método 2 \\
$x$ & $y_{1}$ & $y_{2}$ & $x$ & $y_{1}$ & $y_{2}$ \\
\hline 4.30 & 4.06 & 4.14 & 4.11 & 4.23 & 4.07 \\
4.51 & 4.35 & 4.53 & 3.97 & 3.98 & 4.19 \\
4.42 & 4.52 & 4.57 & 4.40 & 4.36 & 4.33 \\
4.90 & 4.67 & 4.75 & 4.24 & 4.30 & 4.36 \\
4.60 & 4.70 & 4.84 & 4.51 & 4.52 & 4.57 \\
4.29 & 4.30 & 4.21 & 4.49 & 4.33 & 4.61 \\
4.30 & 4.47 & 4.01 & 4.42 & 4.14 & 4.22 \\
4.69 & 4.59 & 4.47 & 4.49 & 4.59 & 4.69 \\
4.02 & 4.05 & 4.09 & 4.14 & 4.32 & 4.45 \\
4.61 & 4.78 & 4.60 & 4.38 & 4.25 & 4.44 \\
4.35 & 4.25 & 4.60 & 4.45 & 4.60 & 4.28 \\
4.46 & 4.55 & 4.70 & 4.39 & 4.38 & 4.64 \\
4.08 & 4.12 & 3.85 & 4.56 & 4.35 & 4.83 \\
3.95 & 3.95 & 4.13 & 4.38 & 4.40 & 4.60 \\
4.21 & 4.27 & 3.94 & 4.44 & 4.34 & 4.66 \\
4.62 & 4.47 & 4.50 & 4.21 & 4.24 & 4.07 \\
4.81 & 4.81 & 4.98 & 4.51 & 4.52 & 4.54 \\
4.47 & 4.34 & 4.33 & 4.23 & 4.12 & 4.35 \\
4.38 & 4.24 & 4.47 & 4.33 & 4.47 & 4.68 \\
4.43 & 4.37 & 4.57 & 4.27 & 4.21 & 4.45 \\
4.74 & 4.52 & 4.58 & 4.67 & 4.52 & 4.60 \\
4.35 & 4.37 & 4.45 & & & \\
\hline
\end{tabular}

Fonte: Jaech (1985). 
A matriz de covariâncias assintótica estimada é dada por

$$
\frac{1}{43} \widehat{\Omega}=\left(\begin{array}{rrrrr}
0.55773 & 0.28491 & -0.12669 & -0.06471 & 1.72 \times 10^{-4} \\
& 0.63277 & -0.06471 & -0.14373 & 1.96 \times 10^{-4} \\
& & 0.02881 & 0.01472 & -3.90 \times 10^{-4} \\
& & & 0.03269 & -4.45 \times 10^{-5} \\
& & & & 5.94 \times 10^{-6}
\end{array}\right) .
$$

Por outro lado, temos que as estimativas de máxima verossimilhança e de momentos para o parâmetro $\beta$, apresentadas na Seção 4.3.3, têm os seguintes valores:

$$
\begin{aligned}
\text { MV }: \widetilde{\beta} & =(0.92157,1.21191)^{\prime}, \\
\text { Momentos : } \beta^{*} & =(0,88558,1.00986)^{\prime} .
\end{aligned}
$$

Note que a estimativa do escore corrigido do parâmetro $\beta$ está mais próxima da estimativa de MV do que a estimativa de momentos.

Finalmente, usando (4.52) e (4.54), temos que o valor da estatística de Wald é $W_{c}=$ 10.46 e da estatística escore é $Q_{c}=10.62$, com quatro graus de liberdade. Ou seja, ao nível de $5 \%$ concluímos que os métodos 1 e 2 medem com vício a densidade de urânio sintetizado. O valor da estatística de Wald baseada no estimador de máxima verossimilhança é 11.38. 


\section{Capítulo 5}

\section{O modelo de regressão Weibull}

\subsection{Introdução}

$\mathrm{Na}$ medicina, na engenharia e em outras áreas de pesquisa, são de grande interesse os métodos estatísticos para avaliar o efeito das covariáveis $z=\left(z_{1}, \ldots, z_{p}\right)^{\prime}$ no tempo de falha ou sobrevivência $T$. A falha refere-se à ocorrência de um evento de interesse, como por exemplo, morte durante um tratamento médico ou falha de um produto num teste de vida industrial acelerado. Por tempo de falha, nos referimos ao período de tempo até a ocorrência do evento. Usualmente, dados de tempo de falha estão sujeitos a censura, ou seja, alguns sujeitos ou itens não falharam até a finalização do estudo. Assumimos aqui que os mecanismos de censura e falha são independentes.

Freqüentemente, as covariáveis consideradas no estudo não podem ser medidas exatamente, como por exemplo, avaliação do estado de uma doença, variáveis biológicas como pressão sangüínea e consumo diário de gorduras saturadas, e medições de quantidades físicas.

O modelo de regressão Weibull tem grande importância entre os métodos paramétricos para a análise de tempos de falha, uma vez que permite modelar tanto taxas de falha crescentes quanto decrescentes como constantes. Em conseqüência dos erros de medição, o estimador "naive" dos parâmetros de interesse é assintoticamente viciado. 
Neste capítulo estudamos o modelo de regressão Weibull estrutural com erros nas variáveis, na presença de censura. Mostramos como o estimador do escore corrigido pode ser obtido para este modelo e propomos um novo estimador que é consistente na ausência de censura. Este estimador é baseado na correção do vício assintótico do estimador "naive". Na Seção 5.2 apresentamos estes estimadores, assim como outros propostos na literatura e analisamos seu comportamento mediante um estudo de simulação. As simulações mostram empiricamente que este novo estimador também se comporta bem na presença de censuras.

Alguns resultados em testes de hipóteses, que estendem aqueles apresentados no Capítulo 3, são obtidos na Seção 5.3.

\subsection{Estimação}

O modelo de regressão Weibull para o $i$-ésimo tempo de falha $T_{i}$ de uma amostra de $n$ observações é usualmente escrito em termos do seu logaritmo como

$$
\log T_{i}=\alpha+\beta^{\prime} z_{i}+\phi \varepsilon_{i}, \quad i=1, \ldots, n,
$$

onde $z_{i}$ é o vetor $p \times 1$ de covariáveis, $\alpha$ e $\beta$ são vetores $p \times 1$ de parâmetros, $\phi$ é o parâmetro de escala e $\varepsilon_{i}$ é um erro aleatório com distribuição valor extremo padrão, cuja densidade é dada por

$$
f\left(\varepsilon_{i}\right)=e^{\varepsilon_{i}} \exp \left(-e^{\varepsilon_{i}}\right) .
$$

Consideremos o mecanismo de censura aleatória, onde

$$
C_{1}, C_{2}, \ldots, C_{n} \text { são v.a.i.i.d., } \operatorname{com} C_{i} \text { independente de } T_{i},
$$

representando os tempos de censura.

Estamos interessados na situação em que $z_{i}$ não pode ser medida exatamente e no lugar de $z_{i}$ observamos

$$
x_{i}=z_{i}+u_{i}, \quad i=1, \ldots, n,
$$


onde $u_{i} \stackrel{\text { iid }}{\sim} N\left(0, \Sigma_{u}\right)$, independente de $z_{i}$ e $y_{i}$, e $z_{i} \stackrel{\text { iid }}{\sim} N\left(\mu, \Sigma_{z}\right)$. Assumimos que $\Sigma_{u}$ é conhecida ou pode ser estimada.

Os dados observados são $\left(y_{i}, \delta_{i}\right), i=1, \ldots, n$, onde

$$
\begin{aligned}
y_{i} & =\min \left(\log T_{i}, \log C_{i}\right) \\
\mathrm{e} \quad \delta_{i} & = \begin{cases}1 & \text { se } T_{i} \leq C_{i} \\
0 & \text { se } T_{i}>C_{i}\end{cases}
\end{aligned}
$$

é o indicador de falha.

Quando as variáveis $z_{i}$ são realmente observadas, a estimação de $\theta=\left(\alpha, \beta^{\prime}, \phi\right)^{\prime}$ é baseada na função de log-verossimilhança

$$
\begin{aligned}
\ell(\theta ; Z, Y) & =\sum_{i=1}^{n} \ell_{i}\left(\theta ; z_{i}, y_{i}\right) \\
& =\sum_{i=1}^{n}\left\{\delta_{i}\left(\frac{y_{i}-\alpha-\beta^{\prime} z_{i}}{\phi}-\log \phi\right)-\exp \left(\frac{y_{i}-\alpha-\beta^{\prime} z_{i}}{\phi}\right)\right\} .
\end{aligned}
$$

O EMV de $\theta$ é obtido maximizando (5.5), o que equivale a resolver a equação $U(\theta ; Z, Y)$ $=\sum_{i=1}^{n} U_{i}\left(\theta ; z_{i}, y_{i}\right)=0, \operatorname{com} U_{i}=\left(U_{i \alpha}, U_{i \beta}^{\prime}, U_{i \phi}\right)^{\prime}$, onde

$$
\begin{aligned}
U_{i \alpha}(\theta)= & \frac{\partial \ell_{i}(\theta)}{\partial \alpha}=\frac{1}{\phi}\left[\exp \left(\frac{y_{i}-\alpha-\beta^{\prime} z_{i}}{\phi}\right)-\delta_{i}\right] \\
U_{i \beta}(\theta)= & \frac{\partial \ell_{i}(\theta)}{\partial \beta}=\frac{1}{\phi}\left[\exp \left(\frac{y_{i}-\alpha-\beta^{\prime} z_{i}}{\phi}\right)-\delta_{i}\right] z_{i}, \\
U_{i \phi}(\theta)= & \frac{\partial \ell_{i}(\theta)}{\partial \phi}=-\delta_{i}\left[\frac{1}{\phi}+\frac{y_{i}-\alpha-\beta^{\prime} z_{i}}{\phi^{2}}\right] \\
& +\exp \left(\frac{y_{i}-\alpha-\beta^{\prime} z_{i}}{\phi}\right)\left(\frac{y_{i}-\alpha-\beta^{\prime} z_{i}}{\phi^{2}}\right), i=1, \ldots, n .
\end{aligned}
$$

Denotamos por $\hat{\theta}_{Z}$ ao estimador de $\theta$ obtido como solução de $U(\theta ; Z, Y)=0$ e por $\widehat{\theta}_{X}$ ao estimador "naive", ou seja, a solução de $U(\theta ; X, Y)=0$, quando as verdadeiras covariáveis $Z$ são substituídas pelas covariáveis observadas $X$. É sabido que, em geral $\widehat{\theta}_{X}$ é assintoticamente viciado (Stefanski, 1995). A seguir mostramos como o método do escore corrigido pode ser aplicado para a obtenção de estimadores consistentes e assintoticamente normais para o parâmetro $\theta$. 


\subsubsection{O estimador do escore corrigido}

Aplicando as propriedades (2.35) - (2.37) da função geradora de momentos de uma variável normal, segue a partir das equações (5.6) - (5.8) que a função escore corrigida é dada por $U^{*}(\theta ; X, Y)=\sum_{i=1}^{n} U_{i}^{*}\left(\theta ; x_{i}, y_{i}\right), \operatorname{com} U_{i}^{*}=\left(U_{i \alpha}^{*}, U_{i \beta}^{*^{\prime}}, U_{i \phi}^{*}\right)^{\prime}$, onde

$$
\begin{aligned}
U_{i \alpha}^{*}(\theta) & =\frac{1}{\phi}\left[\exp \left(\frac{y_{i}-\alpha-\beta^{\prime} x_{i}}{\phi}-\xi\right)-\delta_{i}\right] \\
U_{i \beta}^{*}(\theta) & =\frac{1}{\phi}\left[\exp \left(\frac{y_{i}-\alpha-\beta^{\prime} x_{i}}{\phi}-\xi\right)-\delta_{i}\right] x_{i}+\frac{1}{\phi^{2}} \exp \left(\frac{y_{i}-\alpha-\beta^{\prime} x_{i}}{\phi}-\xi\right) \Sigma_{u} \beta \\
U_{i \phi}^{*}(\theta) & =-\delta_{i}\left[\frac{1}{\phi}+\frac{y_{i}-\alpha-\beta^{\prime} x_{i}}{\phi^{2}}\right]+\exp \left(\frac{y_{i}-\alpha-\beta^{\prime} x_{i}}{\phi}-\xi\right)\left(\frac{y_{i}-\alpha-\beta^{\prime} x_{i}}{\phi^{2}}-\frac{\beta^{\prime} \Sigma_{u} \beta}{\phi^{3}}\right)
\end{aligned}
$$

onde $\xi=\frac{\beta^{\prime} \Sigma_{u} \beta}{2 \phi^{2}}$.

O estimador do escore corrigido, que denotamos por $\widehat{\theta}_{c}$, é solução da equação $U^{*}(\theta ; X, Y)=0$, ou seja, $\widehat{\theta}_{c}=\left(\widehat{\alpha}_{c}, \widehat{\beta}_{c}^{\prime}, \widehat{\phi}_{c}\right)^{\prime}$ é solução do sistema de equações

$$
\begin{aligned}
& \sum_{i=1}^{n} \exp \left(\frac{y_{i}-\alpha-\beta^{\prime} x_{i}}{\phi}-\xi\right)-r=0 \\
& -\sum_{N C} x_{i}+\frac{r \Sigma_{u} \beta}{\phi}+\sum_{i=1}^{n} \exp \left(\frac{y_{i}-\alpha-\beta^{\prime} x_{i}}{\phi}-\xi\right) x_{i}=0 \\
& -r-\frac{1}{\phi} \sum_{N C} y_{i}+\frac{1}{\phi} \sum_{i=1}^{n} \exp \left(\frac{y_{i}-\alpha-\beta^{\prime} x_{i}}{\phi}-\xi\right) y_{i}=0
\end{aligned}
$$

onde $r=\sum_{i=1}^{n} \delta_{i}$ (número total de falhas) e $\sum_{N C}$ denota somatória sobre as observações não censuradas.

Note que a função de log-verossimilhança corrigida é dada por

$$
\begin{aligned}
\ell^{*}(\theta ; X, Y) & =\sum_{i=1}^{n} \ell_{i}^{*}\left(\theta ; x_{i}, y_{i}\right) \\
& =\delta_{i}\left(\frac{y_{i}-\alpha-\beta^{\prime} x_{i}}{\phi}-\log \phi\right)-\exp \left(\frac{y_{i}-\alpha-\beta^{\prime} x_{i}}{\phi}-\xi\right)
\end{aligned}
$$

$\mathrm{e}$

$$
U_{i}^{*}\left(\theta ; x_{i}, y_{i}\right)=\frac{\partial \ell_{i}^{*}}{\partial \theta}\left(\theta ; x_{i}, y_{i}\right)
$$

de modo que $\hat{\theta}_{c}$ é a solução de $U^{*}(\theta ; X, Y)=0$, que maximiza $\ell^{*}(\theta ; X, Y)$. 


\subsubsection{Alguns estimadores propostos na literatura}

A seguir apresentamos resumidamente alguns dos métodos propostos na literatura para corrigir o vício de estimadores em modelos com erros nas variáveis. Em princípio, assumimos que $\Sigma_{u}$ conhecida.

\section{O método de regressão-calibração}

O método é proposto de maneira geral no trabalho de Carroll e Stefanski (1990). A idéia básica consiste em substituir a variável não observada $z$ por um estimador da esperança condicional de $z$ dado $x$ (métodos de estimação desta esperança condicional são discutidos em Carroll et al., 1995). Após esta aproximação, uma análise usual é realizada. O método é simples e potencialmente aplicável a qualquer modelo de regressão, desde que a aproximação considerada seja suficientemente boa.

Quando $u \sim N\left(0, \Sigma_{u}\right)$ e $z \sim N\left(\mu, \Sigma_{z}\right)$, temos que

$$
E[z \mid x]=\mu+\Sigma_{z}\left(\Sigma_{z}+\Sigma_{u}\right)^{-1}(x-\mu)
$$

sendo estimada por

$$
\widehat{E}[z \mid x]=\widehat{\mu}+\left(\widehat{\Sigma}_{x}-\Sigma_{u}\right) \widehat{\Sigma}_{x}^{-1}(x-\widehat{\mu}),
$$

onde

$$
\widehat{\mu}=\bar{x}=\frac{1}{n} \sum_{i=1}^{n} x_{i} \quad \text { e } \quad \widehat{\Sigma}_{x}=\frac{1}{n-1} \sum_{i=1}^{n}\left(x_{i}-\bar{x}\right)\left(x_{i}-\bar{x}\right)^{\prime} .
$$

Denotamos por $\hat{\theta}_{R}$ ao estimador do parâmetro $\theta$, obtido como solução de $\sum_{i=1}^{n} U_{i}(\theta$; $\left.\widehat{E}\left[z_{i} \mid x_{i}\right], y_{i}\right)=0$, com $\widehat{E}\left[z_{i} \mid x_{i}\right]$ dada por (5.12). Maiores detalhes sobre o método de regressão-calibração podem ser encontrados nos trabalhos de Carroll e Stefanski (1990), Gleser (1990) e Carroll et al. (1995). 


\section{O método baseado em estimadores de James-Stein}

A mesma idéia de substituir a covariável não observada por um estimador é considerada em Whittemore (1989). z é estimada pelo estimador de James-Stein

$$
\widehat{z}=\widehat{z}(x)=\widehat{B} \bar{x}+(1-\widehat{B}) x
$$

onde $\widehat{B}=(n-3) \Sigma_{u} S^{-1}$ com $S=\sum_{i=1}^{n}\left(x_{i}-\bar{x}\right)\left(x_{i}-\bar{x}\right)^{\prime}$. Efron e Morris $(1973,1975)$ mostram que o estimador de James-Stein aparece naturalmente num contexto bayesiano empírico.

O método produz estimadores consistentes dos parâmetros de regressão no modelo clássico de regressão linear com erros nas variáveis e se aplica a uma ampla classe de problemas de regressão não lineares, desde que o erro de medição seja normalmente distribuído com variância conhecida.

Note que (5.12) pode ser escrito como

$$
\widehat{E}[z \mid x]=\widehat{B}^{*} \bar{x}+\left(1-\widehat{B}^{*}\right) x
$$

com $\widehat{B}^{*}=(n-1) \Sigma_{u} S^{-1}$, de modo que (5.12) e (5.13) têm a mesma forma, a menos de uma pequena diferença entre $\widehat{B}$ e $\widehat{B}^{*}$. Denotamos por $\hat{\theta}_{W}$ ao estimador de $\theta$, obtido como solução de $\sum_{i=1}^{n} U_{i}\left(\theta ; \widehat{z}_{i}\left(x_{i}\right), y_{i}\right)=0$, com $\widehat{z}_{i}\left(x_{i}\right)$ dado por $(5.13)$.

\section{Correção do vício através de expansões de Taylor}

Stefanski (1985) propõe um método diferente para reduzir o vício assintótico do estimador "naive", baseado em uma expansão em série de Taylor.

Com a finalidade de simplificar a exposição, consideramos aqui $x$ e $\theta$ unidimensionais (o caso multidimensional é conceitualmente idêntico, embora mais complicado na notação). Note que, se $u \sim N\left(0, \sigma_{u}^{2}\right)$, então podemos escrever

$$
x=z+\sigma_{u} e,
$$


onde $e \sim N(0,1)$.

O estimador "naive" de $\theta, \hat{\theta}_{X}$ não converge para o verdadeiro valor do parâmetro $\theta_{0}$, mas a um valor $\theta\left(\sigma_{u}\right)$ que satisfaz

$$
E\left[U_{i}\left(\theta\left(\sigma_{u}\right) ; x_{i}, y_{i}\right)\right]=0, \quad i=1, \ldots, n,
$$

onde a esperança é tomada sob o verdadeiro valor do parâmetro $\theta_{0}$. Geralmente, $\theta\left(\sigma_{u}\right) \neq \theta_{0}$ e, conseqüentemente, $\widehat{\theta}_{X}$ é assintoticamente viciado.

A equação (5.14) define $\theta\left(\sigma_{u}\right)$ implicitamente como função de $\sigma_{u}$. Para o modelo de regressão Weibull considerado neste capítulo, na ausência de censura, $\theta\left(\sigma_{u}\right)$ pode ser obtido explicitamente. Baseado nesse resultado, na Seção 5.2 .3 é proposto um novo estimador.

A expressão (5.14) pode ser usada para expandir $\theta\left(\sigma_{u}\right)$ em série de Taylor em torno do ponto $\sigma_{u}=0$ (note que $\theta(0)=\theta_{0}$ ). Diferenciando (5.14) em relação a $\sigma_{u}$ e assumindo condições de regularidade convenientes, temos que

$$
E\left[U_{2}\left(\theta\left(\sigma_{u}\right) ; z+\sigma_{u} e, y\right) e+U_{1}\left(\theta\left(\sigma_{u}\right) ; z+\sigma_{u} e, y\right) \theta^{(1)}\left(\sigma_{u}\right)\right]=0,
$$

onde as derivadas parciais são denotadas com subíndices, i.e., $U_{2}(\theta ; z, y)=\frac{\partial}{\partial z} U(\theta ; z, y) \mathrm{e}$ $U_{1}(\theta ; z, y)=\frac{\partial}{\partial \theta} U(\theta ; z, y)$; com supraíndices denotamos as derivadas $\theta^{(k)}\left(\sigma_{u}\right)=\mathrm{d}^{k} \theta / \mathrm{d} \sigma_{u}^{k}$. Avaliando (5.15) em $\sigma_{u}=0$, é fácil ver que $\theta^{(1)}(0)=0$.

Novamente, uma diferenciação de (5.15) e avaliação em $\sigma_{u}=0$, produz

$$
\theta^{(2)}(0)=-A^{-1} E\left[U_{22}\left(\theta_{0} ; z, y\right)\right]
$$

onde $A=E\left[\frac{\partial}{\partial \theta} U\left(\theta_{0} ; z, y\right)\right]$.

Portanto, para $\sigma_{u}$ pequeno, temos que

$$
\theta_{0}=\theta^{*}\left(\sigma_{u}\right)+o\left(\sigma_{u}^{2}\right)
$$

onde

$$
\theta^{*}\left(\sigma_{u}\right)=\theta\left(\sigma_{u}\right)+\frac{1}{2} \sigma_{u}^{2} A^{-1} E\left[U_{22}\left(\theta_{0} ; z, y\right)\right] .
$$


Note que $\theta^{*}\left(\sigma_{u}\right)$ está mais próximo de $\theta_{0}$ que $\theta\left(\sigma_{u}\right)$, mas ainda é função de $\theta_{0}$ e dos verdadeiros $z_{i}$. Um estimador de $\theta^{*}\left(\sigma_{u}\right)$ pode ser definido por

$$
\hat{\theta}_{S}\left(\sigma_{u}\right)=\hat{\theta}_{X}+\frac{1}{2} \sigma_{u}^{2}\left\{\sum_{i=1}^{n} U_{1}\left(\hat{\theta}_{X} ; x_{i}, y_{i}\right)\right\}^{-1}\left\{\sum_{i=1}^{n} U_{22}\left(\hat{\theta}_{X} ; x_{i}, y_{i}\right)\right\} .
$$

$\hat{\theta}_{S}\left(\sigma_{u}\right)$ converge para $\theta_{S}\left(\sigma_{u}\right)$, em geral $\theta_{S}\left(\sigma_{u}\right) \neq \theta^{*}\left(\sigma_{u}\right)$, mas é simples mostrar que $\theta_{S}\left(\sigma_{u}\right)=\theta_{0}+o\left(\sigma_{u}^{2}\right)$. Segue então que, para $\sigma_{u}$ pequeno, $\theta_{S}\left(\sigma_{u}\right)$ é aproximadamente igual a $\theta_{0}$ e é consistentemente estimado por $\hat{\theta}_{S}\left(\sigma_{u}\right)$.

Portanto, para o modelo de regressão Weibull, um estimador "aproximadamente consistente" é dado por

$$
\widehat{\theta}_{S}=\widehat{\theta}_{X}+\frac{1}{2} \sigma_{u}^{2}\left\{\sum_{i=1}^{n} \frac{\partial}{\partial \theta^{\prime}} U_{i}\left(\hat{\theta}_{X} ; x_{i}, y_{i}\right)\right\}^{-1}\left\{\sum_{i=1}^{n} \frac{\partial^{2}}{\partial x_{i}^{2}} U_{i}\left(\hat{\theta}_{X} ; x_{i}, y_{i}\right)\right\},
$$

onde $U_{i}$ é o escore dado em (5.6) - (5.8) e $\widehat{\theta}_{X}$ é o estimador "naive".

\subsubsection{Novo estimador}

Mostramos a seguir que, na ausência de censura, o vício assintótico do estimador "naive" do parâmetro $\beta$ pode ser obtido explicitamente. Conseqüentemente, um novo estimador do parâmetro $\beta$, baseado na correção do vício, pode então ser proposto.

Com efeito, (5.14) implica que

$$
\begin{aligned}
& E\left[U_{i \alpha}\left(\theta\left(\Sigma_{u}\right) ; x_{i}, y_{i}\right)\right]=0 \\
& E\left[U_{i \beta}\left(\theta\left(\Sigma_{u}\right) ; x_{i}, y_{i}\right)\right]=0
\end{aligned}
$$

onde a esperança é tomada sob o verdadeiro valor do parâmetro $\theta_{0}=\left(\alpha_{0}, \beta_{0}^{\prime}, \phi_{0}\right)^{\prime}$, com $U_{i \alpha}$ e $U_{i \beta}$ definidas em (5.6) e (5.7), respectivamente.

De maneira análoga à prova do Lema 3.2, usando propriedades da função geradora de momentos de uma variável normal, segue a partir de (5.16) e (5.17) que

$$
\begin{aligned}
& -1+\exp \left[\frac{\left(\alpha_{0}-\alpha\right)+\left(\beta_{0}-\beta\right)^{\prime} \mu}{\phi}+\xi+\gamma\right] \Gamma\left(\frac{\phi_{0}}{\phi}+1\right)=0 \\
& -\mu+\exp \left[\frac{\left(\alpha_{0}-\alpha\right)+\left(\beta_{0}-\beta\right)^{\prime} \mu}{\phi}+\xi+\gamma\right] \Gamma\left(\frac{\phi_{0}}{\phi}+1\right)\left[\mu+\frac{1}{\phi} \Sigma_{z}\left(\beta_{0}-\beta\right)-\frac{1}{\phi} \Sigma_{u} \beta\right]=0 .
\end{aligned}
$$


Resolvendo (5.18) e (5.19), encontramos

$$
\beta\left(\Sigma_{u}\right)=R \beta_{0}
$$

onde $R=\left(\Sigma_{z}+\Sigma_{u}\right)^{-1} \Sigma_{z} \beta_{0}$.

Concluímos que, no modelo de regressão Weibull sem censura, o erro de medição atenua o estimador do parâmetro $\beta$, da mesma forma que no modelo de regressão linear. O mesmo resultado foi encontrado para a classe de modelos considerados na Seção 3.4.

A partir do resultado que $\widehat{\beta}_{X} \stackrel{\mathrm{p}}{\longrightarrow} R \beta_{0}$, podemos definir um estimador para o parâmetro $\beta$, que é consistente na ausência de censura, como

$$
\widehat{\beta}_{N}=\widehat{R}^{-1} \widehat{\beta}_{X}
$$

onde $\widehat{R}=\widehat{\Sigma}_{x}^{-1}\left(\widehat{\Sigma}_{x}-\Sigma_{u}\right) \operatorname{com} \widehat{\Sigma}_{x}=\frac{1}{n-1} \sum_{i=1}^{n}\left(x_{i}-\bar{x}\right)\left(x_{i}-\bar{x}\right)^{\prime}$.

Observação: Quando $\Sigma_{u}$ é desconhecida, são necessárias medidas repetidas das covariáveis $z_{i}$ para podermos estimá-la. Ou seja, se $x_{i j}$ é a $j$-ésima medida repetida de $z_{i}$, $j=1, \ldots, k_{i}$, assumindo

$$
x_{i j}=z_{i}+u_{i j}, \quad j=1, \ldots, k_{i}, i=1, \ldots, n
$$

onde $u_{i j} \stackrel{\text { iid }}{\sim} N\left(0, \Sigma_{u}\right)$, independentes de $z_{i}, \Sigma_{u}$ pode ser estimada consistentemente pelo seguinte estimador da análise de componentes de variância,

$$
\widehat{\Sigma}_{u}=\frac{\sum_{i=1}^{n} \sum_{i=1}^{k_{i}}\left(x_{i j}-\bar{x}_{i}\right)\left(x_{i j}-\bar{x}_{i \cdot}\right)^{\prime}}{\sum_{i=1}^{n}\left(k_{i}-1\right)},
$$

como mencionado na Seção 2.4. No cálculo dos estimadores antes definidos, substituímos $x_{i}$ por $\bar{x}_{i}, \Sigma_{u}$ por $\Sigma_{u} / k_{i}$ e $\bar{x}$ por $\bar{x} \ldots$

\subsubsection{Um estudo de simulação}

Nesta seção apresentamos um estudo de simulação de Monte Carlo comparando o comportamento dos estimadores do parâmetro $\beta$, definidos nas seções anteriores. 
O estudo é baseado num modelo de regressão Weibull com uma única covariável e parâmetro de forma igual a 2 .

A verdadeira covariável $z_{i}$ foi gerada como uma normal padrão, i.e., $z_{i} \sim N(0,1)$.

A covariável observada $x_{i}$ foi gerada condicionada na verdadeira covariável $z_{i}$, segundo o modelo $x_{i}=z_{i}+u_{i}$, onde $u_{i} \sim N\left(0, \sigma_{u}^{2}\right)$.

A resposta $T_{i}$, condicionada em $z_{i}$ foi gerada como uma variável aleatória Weibull $\left(2, \exp \left(\alpha+\beta z_{i}\right)\right), \operatorname{com} \alpha=0$ e $\beta=1$.

Uma variável $C_{i}$, independente de $T_{i}$, correspondente ao mecanismo de censura, foi gerada como uma variável uniforme no intervalo $(0, \tau)$. A proporção de censura $P\left(C_{i}<T_{i}\right)$ foi obtida controlando o valor do parâmetro $\tau$. Os dados observados $y_{i}=\min \left(T_{i}, C_{i}\right)$ e $\delta_{i}$ foram registrados, onde $\delta_{i}=1$ se $T_{i} \leq C_{i}$ e $\delta_{i}=0$ se $T_{i}>C_{i}$.

Várias combinações de tamanhos amostrais $(n=50,100,300)$, a variância do erro de medição $\left(\sigma_{u}^{2}=0.1,0.3,0.5\right)$ e a proporção de censura $(F=0 \%, 25 \%, 50 \%)$ na amostra foram consideradas no estudo.

Mil replicações foram geradas para cada simulação. Nas Tabelas 5.1, 5.2 e 5.3 apresentamos as médias, desvio padrão (DP) e a raiz quadrada do erro quadrático médio (REQM) dos diferentes estimadores de $\beta$. Nas Tabelas 5.4, 5.5 e 5.6, os estimadores são obtidos com $\sigma_{u}^{2}$ sendo estimada a partir de duas réplicas para cada $z_{i}$. A seguinte notação é utilizada:

$\widehat{\beta}_{Z}:$ EMV baseado nos dados verdadeiros $\left(y_{1}, z_{1}\right), \ldots,\left(y_{n}, z_{n}\right) ;$

$\widehat{\beta}_{X}:$ EMV "naive";

$\widehat{\beta}_{N}$ : novo estimador, definido em (5.20);

$\widehat{\beta}_{c}$ : estimador do escore corrigido;

$\widehat{\beta}_{S}$ : estimador proposto em Stefanski (1985), mediante correção do vício por expansões de Taylor;

$\widehat{\beta}_{W}$ : estimador proposto em Whittemore (1989), baseado em estimadores de 


\section{James-Stein;}

$\widehat{\beta}_{R}$ : estimador do método de regressão-calibração. 
Tabela 5.1 - Estimativas do parâmetro $\beta$ (sem censura)

\begin{tabular}{|c|c|c|c|c|c|c|c|c|c|}
\hline \multirow[b]{2}{*}{ Estimador } & \multicolumn{3}{|c|}{$n=50$} & \multicolumn{3}{|c|}{$n=100$} & \multicolumn{3}{|c|}{$n=300$} \\
\hline & Média & DP & REQM & Média & DP & REQM & Média & DP & REQM \\
\hline$\widehat{\beta}_{Z}$ & 1.00 & 0.30 & 0.30 & 1.00 & 0.20 & 0.20 & 1.00 & 0.12 & 0.12 \\
\hline \multicolumn{10}{|l|}{$\sigma_{u}^{2}=0.1$} \\
\hline$\widehat{\beta}_{X}$ & 0.90 & 0.28 & 0.30 & 0.90 & 0.19 & 0.22 & 0.91 & 0.12 & 0.15 \\
\hline$\widehat{\beta}_{N}$ & 0.99 & 0.31 & 0.31 & 0.99 & 0.21 & 0.21 & 1.01 & 0.13 & 0.13 \\
\hline$\widehat{\beta}_{c}$ & 0.99 & 0.31 & 0.31 & 0.99 & 0.21 & 0.21 & 1.01 & 0.13 & 0.13 \\
\hline$\widehat{\beta}_{S}$ & 0.98 & 0.31 & 0.31 & 0.98 & 0.21 & 0.21 & 1.00 & 0.13 & 0.13 \\
\hline$\widehat{\beta}_{W}$ & 0.98 & 0.31 & 0.31 & 0.98 & 0.21 & 0.21 & 1.01 & 0.13 & 0.13 \\
\hline$\widehat{\beta}_{R}$ & 0.99 & 0.31 & 0.31 & 0.99 & 0.21 & 0.21 & 1.01 & 0.13 & 0.13 \\
\hline \multicolumn{10}{|l|}{$\sigma_{u}^{2}=0.3$} \\
\hline$\widehat{\beta}_{X}$ & 0.75 & 0.26 & 0.36 & 0.75 & 0.18 & 0.31 & 0.76 & 0.11 & 0.27 \\
\hline$\widehat{\beta}_{N}$ & 0.96 & 0.33 & 0.34 & 0.98 & 0.23 & 0.23 & 1.00 & 0.14 & 0.14 \\
\hline$\widehat{\beta}_{c}$ & 0.97 & 0.33 & 0.33 & 0.98 & 0.24 & 0.24 & 1.00 & 0.15 & 0.15 \\
\hline$\widehat{\beta}_{S}$ & 0.92 & 0.33 & 0.33 & 0.93 & 0.22 & 0.24 & 0.94 & 0.14 & 0.15 \\
\hline$\widehat{\beta}_{W}$ & 0.95 & 0.33 & 0.33 & 0.97 & 0.23 & 0.23 & 1.00 & 0.14 & 0.14 \\
\hline$\widehat{\beta}_{R}$ & 0.96 & 0.33 & 0.33 & 0.97 & 0.23 & 0.23 & 1.00 & 0.14 & 0.14 \\
\hline \multicolumn{10}{|l|}{$\sigma_{u}^{2}=0.5$} \\
\hline$\widehat{\beta}_{X}$ & 0.64 & 0.24 & 0.43 & 0.65 & 0.17 & 0.39 & 0.65 & 0.10 & 0.37 \\
\hline$\widehat{\beta}_{N}$ & 0.94 & 0.35 & 0.37 & 0.97 & 0.25 & 0.25 & 0.99 & 0.16 & 0.16 \\
\hline$\widehat{\beta}_{c}$ & 0.94 & 0.36 & 0.36 & 0.96 & 0.26 & 0.26 & 0.99 & 0.16 & 0.16 \\
\hline$\widehat{\beta}_{S}$ & 0.86 & 0.33 & 0.36 & 0.86 & 0.23 & 0.26 & 0.87 & 0.14 & 0.19 \\
\hline$\widehat{\beta}_{W}$ & 0.92 & 0.34 & 0.35 & 0.96 & 0.25 & 0.25 & 0.98 & 0.16 & 0.16 \\
\hline$\widehat{\beta}_{R}$ & 0.94 & 0.35 & 0.36 & 0.97 & 0.25 & 0.25 & 0.99 & 0.16 & 0.16 \\
\hline
\end{tabular}


Tabela 5.2 - Estimativas do parâmetro $\beta$ (25\% de censura)

\begin{tabular}{|c|c|c|c|c|c|c|c|c|c|}
\hline \multirow[b]{2}{*}{ Estimador } & \multicolumn{3}{|c|}{$n=50$} & \multicolumn{3}{|c|}{$n=100$} & \multicolumn{3}{|c|}{$n=300$} \\
\hline & Média & DP & REQM & Média & DP & $\overline{R E Q M}$ & Média & $\mathrm{DP}$ & REQM \\
\hline$\widehat{\beta}_{Z}$ & 1.04 & 0.37 & 0.37 & 1.01 & 0.26 & 0.26 & 1.01 & 0.15 & 0.15 \\
\hline \multicolumn{10}{|l|}{$\sigma_{u}^{2}=0.1$} \\
\hline$\widehat{\beta}_{X}$ & 0.95 & 0.35 & 0.36 & 0.92 & 0.25 & 0.26 & 0.91 & 0.14 & 0.16 \\
\hline$\widehat{\beta}_{N}$ & 1.01 & 0.38 & 0.38 & 0.99 & 0.27 & 0.27 & 1.01 & 0.16 & 0.16 \\
\hline$\widehat{\beta}_{c}$ & 1.05 & 0.40 & 0.40 & 1.02 & 0.28 & 0.28 & 1.01 & 0.15 & 0.15 \\
\hline$\widehat{\beta}_{S}$ & 1.01 & 0.39 & 0.39 & 0.99 & 0.27 & 0.27 & 1.00 & 0.16 & 0.16 \\
\hline$\widehat{\beta}_{W}$ & 1.01 & 0.38 & 0.38 & 0.99 & 0.27 & 0.27 & 1.01 & 0.16 & 0.16 \\
\hline$\widehat{\beta}_{R}$ & 1.01 & 0.38 & 0.38 & 0.99 & 0.27 & 0.27 & 1.00 & 0.15 & 0.15 \\
\hline \multicolumn{10}{|l|}{$\sigma_{u}^{2}=0.3$} \\
\hline$\widehat{\beta}_{X}$ & 0.81 & 0.33 & 0.38 & 0.77 & 0.23 & 0.32 & 0.76 & 0.13 & 0.27 \\
\hline$\widehat{\beta}_{N}$ & 0.98 & 0.41 & 0.41 & 0.97 & 0.28 & 0.29 & 1.00 & 0.17 & 0.17 \\
\hline$\widehat{\beta}_{c}$ & 1.07 & 0.45 & 0.46 & 1.04 & 0.33 & 0.33 & 1.01 & 0.18 & 0.18 \\
\hline$\widehat{\beta}_{S}$ & 0.95 & 0.40 & 0.41 & 0.93 & 0.28 & 0.29 & 0.95 & 0.17 & 0.17 \\
\hline$\widehat{\beta}_{W}$ & 0.97 & 0.40 & 0.40 & 0.96 & 0.28 & 0.28 & 1.00 & 0.17 & 0.17 \\
\hline$\widehat{\beta}_{R}$ & 0.98 & 0.41 & 0.41 & 0.97 & 0.28 & 0.28 & 1.00 & 0.17 & 0.17 \\
\hline \multicolumn{10}{|l|}{$\sigma_{u}^{2}=0.5$} \\
\hline$\widehat{\beta}_{X}$ & 0.66 & 0.29 & 0.45 & 0.67 & 0.22 & 0.39 & 0.66 & 0.12 & 0.36 \\
\hline$\widehat{\beta}_{N}$ & 0.96 & 0.43 & 0.43 & 0.96 & 0.30 & 0.30 & 0.99 & 0.18 & 0.18 \\
\hline$\widehat{\beta}_{c}$ & 0.95 & 0.43 & 0.43 & 1.05 & 0.37 & 0.37 & 1.02 & 0.21 & 0.21 \\
\hline$\widehat{\beta}_{S}$ & 0.88 & 0.40 & 0.42 & 0.86 & 0.28 & 0.31 & 0.88 & 0.17 & 0.21 \\
\hline$\widehat{\beta}_{W}$ & 0.94 & 0.42 & 0.42 & 0.95 & 0.30 & 0.30 & 0.99 & 0.18 & 0.18 \\
\hline$\widehat{\beta}_{R}$ & 0.96 & 0.43 & 0.43 & 0.96 & 0.30 & 0.30 & 0.99 & 0.18 & 0.18 \\
\hline
\end{tabular}


Tabela 5.3 - Estimativas do parâmetro $\beta$ (50\% de censura)

\begin{tabular}{|c|c|c|c|c|c|c|c|c|c|}
\hline \multirow[b]{2}{*}{ Estimador } & \multicolumn{3}{|c|}{$n=50$} & \multicolumn{3}{|c|}{$n=100$} & \multicolumn{3}{|c|}{$n=300$} \\
\hline & Média & DP & REQM & Média & DP & REQM & Média & DP & REQM \\
\hline$\widehat{\beta}_{Z}$ & 1.06 & 0.47 & 0.47 & 1.03 & 0.33 & 0.33 & 1.01 & 0.19 & 0.19 \\
\hline \multicolumn{10}{|l|}{$\sigma_{u}^{2}=0.1$} \\
\hline$\widehat{\beta}_{X}$ & 0.94 & 0.42 & 0.43 & 0.91 & 0.31 & 0.32 & 0.91 & 0.18 & 0.20 \\
\hline$\widehat{\beta}_{N}$ & 1.02 & 0.48 & 0.48 & 1.00 & 0.34 & 0.34 & 1.00 & 0.19 & 0.19 \\
\hline$\widehat{\beta}_{c}$ & 1.03 & 0.48 & 0.48 & 1.01 & 0.34 & 0.34 & 1.01 & 0.20 & 0.20 \\
\hline$\widehat{\beta}_{S}$ & 1.03 & 0.49 & 0.49 & 1.01 & 0.35 & 0.35 & 1.01 & 0.20 & 0.20 \\
\hline$\widehat{\beta}_{W}$ & 1.02 & 0.47 & 0.47 & 1.00 & 0.34 & 0.34 & 1.00 & 0.19 & 0.19 \\
\hline$\widehat{\beta}_{R}$ & 1.02 & 0.48 & 0.48 & 1.00 & 0.34 & 0.28 & 1.00 & 0.19 & 0.19 \\
\hline \multicolumn{10}{|l|}{$\sigma_{u}^{2}=0.3$} \\
\hline$\widehat{\beta}_{X}$ & 0.78 & 0.38 & 0.43 & 0.75 & 0.26 & 0.36 & 0.75 & 0.16 & 0.30 \\
\hline$\widehat{\beta}_{N}$ & 0.98 & 0.50 & 0.50 & 0.97 & 0.35 & 0.35 & 0.99 & 0.21 & 0.21 \\
\hline$\widehat{\beta}_{c}$ & 0.99 & 0.50 & 0.50 & 0.98 & 0.36 & 0.36 & 1.00 & 0.22 & 0.22 \\
\hline$\widehat{\beta}_{S}$ & 0.96 & 0.51 & 0.51 & 0.94 & 0.35 & 0.36 & 0.95 & 0.21 & 0.22 \\
\hline$\widehat{\beta}_{W}$ & 0.97 & 0.50 & 0.50 & 0.97 & 0.35 & 0.35 & 0.99 & 0.21 & 0.21 \\
\hline$\widehat{\beta}_{R}$ & 0.98 & 0.50 & 0.50 & 0.97 & 0.35 & 0.35 & 0.99 & 0.21 & 0.21 \\
\hline \multicolumn{10}{|l|}{$\sigma_{u}^{2}=0.5$} \\
\hline$\widehat{\beta}_{X}$ & 0.68 & 0.34 & 0.47 & 0.65 & 0.24 & 0.43 & 0.64 & 0.14 & 0.39 \\
\hline$\widehat{\beta}_{N}$ & 0.95 & 0.53 & 0.53 & 0.96 & 0.37 & 0.37 & 0.98 & 0.22 & 0.22 \\
\hline$\widehat{\beta}_{c}$ & 0.95 & 0.51 & 0.51 & 0.95 & 0.38 & 0.38 & 0.99 & 0.23 & 0.23 \\
\hline$\widehat{\beta}_{S}$ & 0.89 & 0.52 & 0.53 & 0.87 & 0.35 & 0.37 & 0.88 & 0.20 & 0.23 \\
\hline$\widehat{\beta}_{W}$ & 0.94 & 0.52 & 0.52 & 0.95 & 0.36 & 0.36 & 0.98 & 0.22 & 0.22 \\
\hline$\widehat{\beta}_{R}$ & 0.95 & 0.53 & 0.53 & 0.95 & 0.37 & 0.37 & 0.98 & 0.22 & 0.22 \\
\hline
\end{tabular}


Tabela 5.4 - Estimativas do parâmetro $\beta$ (sem censura). Duas réplicas para cada $z$.

\begin{tabular}{|c|c|c|c|c|c|c|c|c|c|}
\hline \multirow[b]{2}{*}{ Estimador } & \multicolumn{3}{|c|}{$n=50$} & \multicolumn{3}{|c|}{$n=100$} & \multicolumn{3}{|c|}{$n=300$} \\
\hline & Média & DP & REQM & Média & DP & REQM & Média & DP & REQM \\
\hline$\widehat{\beta}_{Z}$ & 1.00 & 0.30 & 0.30 & 1.00 & 0.20 & 0.20 & 1.00 & 0.12 & 0.12 \\
\hline$\sigma_{u}^{2}=0.1$ & & & & & & & & & \\
\hline$\widehat{\sigma}_{u}^{2}$ & 0.10 & 0.02 & 0.02 & 0.10 & 0.01 & 0.01 & 0.10 & 0.01 & 0.01 \\
\hline$\hat{\beta}_{X}$ & 0.96 & 0.26 & 0.26 & 0.96 & 0.20 & 0.20 & 0.96 & 0.11 & 0.12 \\
\hline$\widehat{\beta}_{N}$ & 1.00 & 0.27 & 0.27 & 1.00 & 0.20 & 0.20 & 1.00 & 0.12 & 0.12 \\
\hline$\widehat{\beta}_{c}$ & 1.01 & 0.28 & 0.28 & 1.01 & 0.21 & 0.21 & 1.00 & 0.12 & 0.12 \\
\hline$\widehat{\beta}_{S}$ & 1.00 & 0.27 & 0.27 & 1.00 & 0.20 & 0.20 & 1.00 & 0.12 & 0.12 \\
\hline$\widehat{\beta}_{W}$ & 1.00 & 0.27 & 0.27 & 1.00 & 0.20 & 0.20 & 1.00 & 0.12 & 0.12 \\
\hline$\widehat{\beta}_{R}$ & 1.00 & 0.27 & 0.27 & 1.00 & 0.20 & 0.20 & 1.00 & 0.12 & 0.12 \\
\hline$\sigma_{u}^{2}=0.3$ & & & & & & & & & \\
\hline$\widehat{\sigma}_{u}^{2}$ & 0.30 & 0.06 & 0.06 & 0.30 & 0.04 & 0.04 & 0.30 & 0.03 & 0.03 \\
\hline$\widehat{\beta}_{X}$ & 0.90 & 0.26 & 0.28 & 0.89 & 0.19 & 0.22 & 0.88 & 0.11 & 0.16 \\
\hline$\widehat{\beta}_{N}$ & 1.01 & 0.29 & 0.29 & 1.01 & 0.22 & 0.22 & 1.01 & 0.13 & 0.13 \\
\hline$\widehat{\beta}_{c}$ & 1.01 & 0.30 & 0.30 & 1.01 & 0.23 & 0.23 & 1.01 & 0.13 & 0.13 \\
\hline$\widehat{\beta}_{S}$ & 1.00 & 0.30 & 0.30 & 1.00 & 0.22 & 0.22 & 0.99 & 0.12 & 0.12 \\
\hline$\widehat{\beta}_{W}$ & 1.00 & 0.29 & 0.29 & 1.00 & 0.22 & 0.22 & 1.00 & 0.13 & 0.13 \\
\hline$\widehat{\beta}_{R}$ & 1.00 & 0.29 & 0.29 & 1.01 & 0.22 & 0.22 & 1.00 & 0.13 & 0.13 \\
\hline$\sigma_{u}^{2}=0.5$ & & & & & & & & & \\
\hline$\widehat{\sigma}_{u}^{2}$ & 0.50 & 0.10 & 0.10 & 0.50 & 0.07 & 0.07 & 0.50 & 0.04 & 0.04 \\
\hline$\widehat{\beta}_{X}$ & 0.84 & 0.25 & 0.30 & 0.82 & 0.19 & 0.26 & 0.80 & 0.12 & 0.23 \\
\hline$\widehat{\beta}_{N}$ & 1.01 & 0.32 & 0.32 & 1.01 & 0.24 & 0.24 & 1.01 & 0.14 & 0.14 \\
\hline$\widehat{\beta}_{c}$ & 1.02 & 0.33 & 0.33 & 1.02 & 0.25 & 0.25 & 1.01 & 0.16 & 0.16 \\
\hline$\widehat{\beta}_{S}$ & 0.99 & 0.31 & 0.31 & 0.98 & 0.21 & 0.21 & 0.97 & 0.13 & 0.13 \\
\hline$\widehat{\beta}_{W}$ & 1.00 & 0.31 & 0.31 & 1.01 & 0.24 & 0.24 & 1.01 & 0.14 & 0.14 \\
\hline$\widehat{\beta}_{R}$ & 1.00 & 0.31 & 0.31 & 1.01 & 0.24 & 0.24 & 1.01 & 0.14 & 0.14 \\
\hline
\end{tabular}


Tabela 5.5 - Estimativas do parâmetro $\beta$ ( $25 \%$ de censura). Duas réplicas para cada $z$.

\begin{tabular}{|c|c|c|c|c|c|c|c|c|c|}
\hline \multirow[b]{2}{*}{ Estimador } & \multicolumn{3}{|c|}{$n=50$} & \multicolumn{3}{|c|}{$n=100$} & \multicolumn{3}{|c|}{$n=300$} \\
\hline & Média & $\mathrm{DP}$ & REQM & Média & DP & REQM & Média & DP & REQM \\
\hline$\widehat{\beta}_{Z}$ & 1.04 & 0.37 & 0.37 & 1.01 & 0.26 & 0.26 & 1.01 & 0.15 & 0.15 \\
\hline$\sigma_{u}^{2}=0.1$ & & & & & & & & & \\
\hline$\widehat{\sigma}_{u}^{2}$ & 0.10 & 0.02 & 0.02 & 0.10 & 0.01 & 0.01 & 0.10 & 0.01 & 0.01 \\
\hline$\beta_{X}$ & 0.98 & 0.32 & 0.32 & 0.97 & 0.24 & 0.24 & 0.95 & 0.13 & 0.14 \\
\hline$\widehat{\beta}_{N}$ & 1.02 & 0.34 & 0.34 & 1.01 & 0.23 & 0.23 & 1.00 & 0.14 & 0.14 \\
\hline$\widehat{\beta}_{c}$ & 1.02 & 0.34 & 0.34 & 1.02 & 0.26 & 0.26 & 1.00 & 0.14 & 0.14 \\
\hline$\widehat{\beta}_{S}$ & 1.02 & 0.34 & 0.34 & 1.02 & 0.26 & 0.26 & 1.00 & 0.14 & 0.14 \\
\hline$\widehat{\beta}_{W}$ & 1.02 & 0.34 & 0.34 & 1.01 & 0.26 & 0.26 & 1.00 & 0.14 & 0.14 \\
\hline$\widehat{\beta}_{R}$ & 1.02 & 0.34 & 0.34 & 1.01 & 0.26 & 0.26 & 1.00 & 0.14 & 0.14 \\
\hline$\sigma_{u}^{2}=0.3$ & & & & & & & & & \\
\hline$\hat{\sigma}_{u}^{2}$ & 0.30 & 0.06 & 0.06 & 0.30 & 0.04 & 0.04 & 0.30 & 0.03 & 0.03 \\
\hline$\beta_{X}$ & 0.91 & 0.32 & 0.33 & 0.89 & 0.24 & 0.26 & 0.87 & 0.13 & 0.18 \\
\hline$\widehat{\beta}_{N}$ & 1.02 & 0.36 & 0.36 & 1.01 & 0.27 & 0.27 & 1.00 & 0.15 & 0.15 \\
\hline$\widehat{\beta}_{c}$ & 1.03 & 0.37 & 0.37 & 1.02 & 0.28 & 0.28 & 1.01 & 0.15 & 0.15 \\
\hline$\widehat{\beta}_{S}$ & 1.02 & 0.37 & 0.37 & 1.01 & 0.28 & 0.28 & 0.99 & 0.15 & 0.15 \\
\hline$\widehat{\beta}_{W}$ & 1.01 & 0.36 & 0.36 & 1.01 & 0.27 & 0.27 & 1.00 & 0.15 & 0.15 \\
\hline$\widehat{\beta}_{R}$ & 1.02 & 0.36 & 0.36 & 1.01 & 0.27 & 0.27 & 1.00 & 0.15 & 0.15 \\
\hline$\sigma_{u}^{2}=0.5$ & & & & & & & & & \\
\hline$\widehat{\sigma}_{u}^{2}$ & 0.50 & 0.10 & 0.10 & 0.50 & 0.07 & 0.07 & 0.50 & 0.04 & 0.04 \\
\hline$\widehat{\beta}_{X}$ & 0.85 & 0.31 & 0.34 & 0.82 & 0.23 & 0.29 & 0.80 & 0.12 & 0.23 \\
\hline$\hat{\beta}_{N}$ & 1.02 & 0.38 & 0.38 & 1.01 & 0.28 & 0.28 & 1.00 & 0.16 & 0.16 \\
\hline$\widehat{\beta}_{c}$ & 1.04 & 0.40 & 0.40 & 1.01 & 0.30 & 0.30 & 1.01 & 0.16 & 0.16 \\
\hline$\widehat{\beta}_{S}$ & 1.02 & 0.39 & 0.39 & 0.99 & 0.29 & 0.29 & 0.97 & 0.15 & 0.16 \\
\hline$\widehat{\beta}_{W}$ & 1.02 & 0.37 & 0.37 & 1.00 & 0.28 & 0.28 & 0.99 & 0.16 & 0.16 \\
\hline$\widehat{\beta}_{R}$ & 1.02 & 0.38 & 0.38 & 1.01 & 0.28 & 0.28 & 1.00 & 0.16 & 0.16 \\
\hline
\end{tabular}


Tabela 5.6 - Estimativas do parâmetro $\beta$ (50\% de censura). Duas réplicas para cada $z$

\begin{tabular}{|c|c|c|c|c|c|c|c|c|c|}
\hline \multirow[b]{2}{*}{ Estimador } & \multicolumn{3}{|c|}{$n=50$} & \multicolumn{3}{|c|}{$n=100$} & \multicolumn{3}{|c|}{$n=300$} \\
\hline & Média & $\overline{\mathrm{DP}}$ & REQM & Média & DP & REQM & Média & DP & REQM \\
\hline$\widehat{\beta}_{Z}$ & 1.06 & 0.47 & 0.47 & 1.03 & 0.33 & 0.33 & 1.01 & 0.19 & 0.19 \\
\hline \multicolumn{10}{|l|}{$\sigma_{u}^{2}=0.1$} \\
\hline$\hat{\sigma}_{u}^{2}$ & 0.10 & 0.02 & 0.02 & 0.10 & 0.01 & 0.01 & 0.10 & 0.01 & 0.01 \\
\hline$\widehat{\beta}_{X}$ & 0.99 & 0.41 & 0.41 & 0.98 & 0.31 & 0.31 & 0.96 & 0.17 & 0.17 \\
\hline$\widehat{\beta}_{N}$ & 1.04 & 0.43 & 0.43 & 1.02 & 0.32 & 0.32 & 1.01 & 0.18 & 0.18 \\
\hline$\widehat{\beta}_{c}$ & 1.04 & 0.43 & 0.43 & 1.03 & 0.32 & 0.32 & 1.01 & 0.19 & 0.19 \\
\hline$\widehat{\beta}_{S}$ & 1.05 & 0.44 & 0.44 & 1.03 & 0.33 & 0.33 & 1.01 & 0.18 & 0.18 \\
\hline$\widehat{\beta}_{W}$ & 1.04 & 0.43 & 0.43 & 1.02 & 0.32 & 0.32 & 1.01 & 0.18 & 0.18 \\
\hline$\widehat{\beta}_{R}$ & 1.04 & 0.43 & 0.43 & 1.02 & 0.32 & 0.32 & 1.01 & 0.18 & 0.18 \\
\hline \multicolumn{10}{|l|}{$\sigma_{u}^{2}=0.3$} \\
\hline$\widehat{\sigma}_{u}^{2}$ & 0.30 & 0.06 & 0.06 & 0.30 & 0.04 & 0.04 & 0.30 & 0.03 & 0.03 \\
\hline$\widehat{\beta}_{X}$ & 0.93 & 0.40 & 0.40 & 0.90 & 0.29 & 0.31 & 0.88 & 0.16 & 0.20 \\
\hline$\widehat{\beta}_{N}$ & 1.04 & 0.45 & 0.45 & 1.02 & 0.33 & 0.33 & 1.00 & 0.19 & 0.19 \\
\hline$\widehat{\beta}_{c}$ & 1.05 & 0.48 & 0.48 & 1.03 & 0.35 & 0.35 & 1.01 & 0.19 & 0.19 \\
\hline$\widehat{\beta}_{S}$ & 1.06 & 0.48 & 0.48 & 1.03 & 0.35 & 0.35 & 1.01 & 0.19 & 0.19 \\
\hline$\widehat{\beta}_{W}$ & 1.04 & 0.45 & 0.45 & 1.02 & 0.33 & 0.33 & 1.00 & 0.19 & 0.19 \\
\hline$\widehat{\beta}_{R}$ & 1.04 & 0.45 & 0.36 & 1.02 & 0.33 & 0.33 & 1.00 & 0.19 & 0.19 \\
\hline \multicolumn{10}{|l|}{$\sigma_{u}^{2}=0.5$} \\
\hline$\widehat{\sigma}_{u}^{2}$ & 0.50 & 0.10 & 0.10 & 0.50 & 0.07 & 0.07 & 0.50 & 0.04 & 0.04 \\
\hline$\widehat{\beta}_{X}$ & 0.87 & 0.38 & 0.41 & 0.83 & 0.28 & 0.33 & 0.81 & 0.16 & 0.25 \\
\hline$\widehat{\beta}_{N}$ & 1.05 & 0.47 & 0.47 & 1.02 & 0.35 & 0.35 & 1.00 & 0.20 & 0.20 \\
\hline$\widehat{\beta}_{c}$ & 1.05 & 0.50 & 0.50 & 1.04 & 0.37 & 0.37 & 1.02 & 0.20 & 0.20 \\
\hline$\widehat{\beta}_{S}$ & 1.06 & 0.50 & 0.50 & 1.02 & 0.36 & 0.36 & 0.99 & 0.20 & 0.20 \\
\hline$\widehat{\beta}_{W}$ & 1.04 & 0.47 & 0.47 & 1.02 & 0.35 & 0.35 & 1.00 & 0.19 & 0.19 \\
\hline$\widehat{\beta}_{R}$ & 1.04 & 0.47 & 0.47 & 1.02 & 0.35 & 0.35 & 1.00 & 0.20 & 0.20 \\
\hline
\end{tabular}


Algumas conclusões podem ser extraídas do estudo de simulação apresentado nas Tabelas $5.1-5.6$.

O tamanho amostral e a proporção de censura parecem não afetar a magnitude do vício dos estimadores. O efeito é exclusivamente sobre o desvio padrão.

Como esperado, o estimador "naive" é atenuado na direção do zero quando $\sigma_{u}^{2}$ cresce. O mesmo parece acontecer com o estimador proposto por Stefanski, mas a uma velocidade muito menor. Os outros estimadores têm um bom desempenho em termos de correção do vício. $\mathrm{O}$ efeito conhecido de que estimadores com menor vício têm um desvio padrão maior, também é observado. Todos os estimadores têm maior variabilidade do que o "naive" e essa variabilidade cresce quando $\sigma_{u}^{2}$ cresce.

Da comparação de estimadores usando o erro quadrático médio, notamos que, quando a variância do erro de medição é pequena $\left(\sigma_{u}^{2}=0.1\right)$, o estimador "naive" tem o menor erro quadrático médio. Nas outras situações, os estimadores corrigidos parecem ser efetivos. Os estimadores $\widehat{\beta}_{W}$ e $\widehat{\beta}_{N}$ parecem ter um comportamento ligeiramente melhor do que os outros.

O estimador $\widehat{\beta}_{S}$ tem o maior vício entre os estimadores corrigidos, principalmente quando $\sigma_{u}^{2}$ cresce, isto era esperado como conseqüência do fato de que a correção é obtida considerando valores pequenos de $\sigma_{u}^{2}$. Por outro lado, os outros estimadores não impõem este tipo de restrições.

A disponibilidade de medições repetidas das verdadeiras covariáveis, que permitem a estimação de $\sigma_{u}^{2}$, parece ser um fato importante a ser levado em consideração. Nas Tabelas 5.4, 5.5. e 5.6, observamos uma redução no vício e no erro quadrático médio de todos os estimadores.

Em geral, todos os estimadores considerados têm um bom desempenho, com exceção do estimador $\hat{\beta}_{S}$ proposto por Stefanski, que parece não ser apropriado na medida em que $\sigma_{u}^{2}$ cresce. Contudo, o novo estimador $\widehat{\beta}_{N}$ é o mais simples de ser obtido. 


\subsection{Testes de hipóteses}

Nesta seção vamos mostrar as propriedades assintóticas dos testes baseados nas verdadeiras covariáveis, "naive" e corrigido, tanto do tipo escore como Wald. Os resultados são equivalentes àqueles provados nas Seções 3.3 e 3.4 .

\subsubsection{Testes escore}

Nesta seção, mostramos que os resultados da Seção 3.3 .1 obtidos sob a suposição de um modelo linear generalizado continuam valendo para o modelo de regressão Weibull dado por $(5.1)-(5.4)$, com $\phi$ conhecido.

Para o modelo Weibull, a função escore não observada é dada por $U(\theta)=\sum_{i=1}^{n} U_{i}(\theta)$, com

$$
\begin{aligned}
& U_{i \alpha}(\theta)=q_{i}\left(\alpha+\beta^{\prime} z_{i}\right), \\
& U_{i \beta}(\theta)=q_{i}\left(\alpha+\beta^{\prime} z_{i}\right) z_{i},
\end{aligned}
$$

onde

$$
q_{i}\left(\alpha+\beta^{\prime} z_{i}\right)=\frac{1}{\phi}\left[\exp \left(\frac{y_{i}-\alpha-\beta^{\prime} z_{i}}{\phi}\right)-\delta_{i}\right], \quad i=1, \ldots, n .
$$

Como mostrado na Seção 3.3.1, as estatísticas escore baseadas nas verdadeiras covariáveis, "naive" e eficiente, para testar $H_{0}: \beta=0$, na presença do parâmetro "nuisance" $\alpha$, são dadas por

$$
\begin{aligned}
& T_{Z}=\sum_{i=1}^{n} q_{i}\left(\widehat{\alpha}_{0}\right) z_{i}, \\
& T_{X}=\sum_{i=1}^{n} q_{i}\left(\widehat{\alpha}_{0}\right) x_{i}, \\
& T_{\tilde{X}}=\sum_{i=1}^{n} q_{i}\left(\widehat{\alpha}_{0}\right) \widehat{E}_{x_{i}}\left[z_{i}\right],
\end{aligned}
$$

respectivamente, onde $\hat{\alpha}_{0}$ satisfaz

$$
\sum_{i=1}^{n} q_{i}\left(\widehat{\alpha}_{0}\right)=0
$$


A proposição a seguir é análoga à Proposição 3.1 .

Proposição 5.1 Sob o modelo (5.1) - (5.4), temos que

(a) sob $H_{0}$,

$$
n^{-1 / 2} T_{Z} \stackrel{\mathcal{D}}{\longrightarrow} N\left(0, C_{Z}\right), n^{-1 / 2} T_{X} \stackrel{\mathcal{D}}{\longrightarrow} N\left(0, C_{X}\right) \text { e } n^{-1 / 2} T_{\widetilde{X}} \stackrel{\mathcal{D}}{\longrightarrow} N\left(0, C_{\tilde{X}}\right) .
$$

(b) sob uma seqüência de alternativas $\beta^{(n)}, n^{1 / 2} \beta^{(n)} \rightarrow \Delta,\|\Delta\|>0$,

$$
\begin{aligned}
& n^{-1 / 2} T_{Z} \stackrel{\mathcal{D}}{\longrightarrow} N\left(m_{Z}, C_{Z}\right), n^{-1 / 2} T_{X} \stackrel{\mathcal{D}}{\longrightarrow} N\left(m_{X}, C_{X}\right) \text { e } n^{-1 / 2} T_{\tilde{X}} \stackrel{\mathcal{D}}{\longrightarrow} N\left(m_{\tilde{X}}, C_{\tilde{X}}\right), \\
& \text { onde } \\
& C_{Z}=c \Sigma_{z}, C_{X}=c\left(\Sigma_{z}+\Sigma_{u}\right), C_{\tilde{X}}=c \operatorname{Var}\left[E_{x}[z]\right], \\
& m_{Z}=\Sigma_{z} \Delta, m_{X}=\Sigma_{z} \Delta, m_{\tilde{X}}=\operatorname{Var}\left[E_{x}[z]\right] \Delta, \\
& \text { com } c=\frac{1}{\phi^{2}} \lim _{n \rightarrow \infty} \frac{1}{n} \sum_{i=1}^{n} E\left[1-\exp \left(-e^{\frac{C_{i}-\alpha}{\phi}}\right)\right]<\infty .
\end{aligned}
$$

Prova: A prova é análoga à da Proposição $3.1, \operatorname{com} q_{i}(\alpha)=\frac{1}{\phi}\left[\exp \left(\frac{y_{i}-\alpha}{\phi}\right)-\delta_{i}\right]$. No entanto, note que agora no cálculo das variâncias de $t_{Z}, t_{X}$ e $t_{\widetilde{X}}$, temos que, por exemplo,

$$
\begin{aligned}
E\left[q_{i}(\alpha) z_{i} \mid z_{i}\right] & =\operatorname{Var}\left[q_{i}(\alpha) \mid z_{i}\right] z_{i} z_{i}^{\prime} \\
& =\frac{1}{\phi^{2}} E\left[\delta_{i}^{2}+\exp \left(2 \frac{\left(y_{i}-\alpha\right)}{\phi}\right)-2 \delta_{i} \exp \left(\frac{y_{i}-\alpha}{\phi}\right) \mid z_{i}\right] z_{i} z_{i}^{\prime} \\
& =\frac{1}{\phi^{2}} E\left[1-\exp \left(-e^{\frac{C_{i}-\alpha}{\phi}}\right)\right] z_{i} z_{i}^{\prime},
\end{aligned}
$$

de modo que

$$
\operatorname{Var}\left[q_{i}(\alpha) z_{i}\right]=\frac{1}{\phi^{2}} E\left[1-\exp \left(-e^{\frac{C_{i}-\alpha}{\phi}}\right)\right] E\left[z_{i} z_{i}^{\prime}\right]
$$

A condição $\frac{1}{n} \sum_{i=1}^{n} \frac{1}{\phi^{2}}\left[1-\exp \left(-e^{\frac{C_{i}-\alpha}{\phi}}\right)\right] \rightarrow c<\infty$, permite a aplicação do Teorema Central do Limite Multivariado, e o resultado da proposição segue como na Proposição 3.1. 
Por outro lado, temos que a estatística escore corrigido $Q_{c}$ definida em (3.6) é dada por

$$
Q_{c}=n^{-1} T_{c}^{\prime} \hat{C}_{c}^{-1} T_{c}
$$

onde

$$
T_{c}=\sum_{i=1}^{n} U_{i \beta}^{*}\left(\hat{\theta}_{c 0}\right),
$$

$\operatorname{com} \hat{\theta}_{c 0}=\left(\hat{\alpha}_{c 0}, 0^{\prime}\right)^{\prime}$ satisfazendo $\sum_{i=1}^{n} U_{i \alpha}^{*}\left(\hat{\theta}_{c 0}\right)=0, U_{i \alpha}^{*}(\theta)$ e $U_{i \beta}^{*}(\theta)$ definidas em $(5.9)$ e (5.10), respectivamente.

O resultado a seguir é análogo ao da Proposição 3.4 .

Proposição 5.2 Sob o modelo (5.1) - (5.4), $T_{c}=T_{X}, \forall n$. Conseqüentemente, $Q_{c}=Q_{X}$, $\forall n$.

Prova: $T_{c}=\sum_{i=1}^{n} U_{i \beta}^{*}\left(\hat{\theta}_{c 0}\right)$, onde $\hat{\theta}_{c 0}=\left(\hat{\alpha}_{c 0}, 0^{\prime}\right)^{\prime}$ satisfaz $\sum_{i=1}^{n} U_{i \alpha}^{*}\left(\hat{\theta}_{c 0}\right)=0$. Agora,

$$
\sum_{i=1}^{n} U_{i \alpha}^{*}\left(\hat{\theta}_{c 0}\right)=\sum_{i=1}^{n} \frac{1}{\phi}\left[\exp \left(\frac{y_{i}-\widehat{\alpha}_{c 0}}{\phi}\right)-\delta_{i}\right]=\sum_{i=1}^{n} U_{i \alpha}\left(\widehat{\theta}_{c 0}\right)=0
$$

e portanto $\hat{\theta}_{c 0}=\hat{\theta}_{0}$. Assim,

$$
\begin{aligned}
T_{c} & =\sum_{i=1}^{n} U_{i \beta}^{*}\left(\hat{\theta}_{c 0}\right)=\sum_{i=1}^{n} U_{i \beta}^{*}\left(\hat{\theta}_{0}\right) \\
& =\sum_{i=1}^{n} \frac{1}{\phi}\left[\exp \left(\frac{y_{i}-\hat{\alpha}_{0}}{\phi}\right)-\delta_{i}\right] x_{i}=T_{X},
\end{aligned}
$$

o que prova a proposição.

\subsubsection{Testes de Wald}

Devido à presença de censura, o vício assintótico do estimador "naive" do parâmetro $\beta$ não pode ser facilmente encontrado, como foi feito na Seção 5.2.3 na ausência de censura. Os efeitos do erro de medição podem ser complexos na presença de dados censurados. Contudo, as simulações apresentadas na Seção 5.4 parecem indicar que o mesmo efeito da atenuação continua ocorrendo na presença de censuras. 
Dado que não dispomos de uma expressão explícita para o vício assintótico do estimador "naive" para valores de $\beta$ distintos de zero, apresentamos nesta seção resultados análogos aos da Seção 3.4, para o modelo Weibull sem censura.

Proposição 5.3 Sob o modelo dado por (5.1) e (5.3), temos que

(a) sob $H_{0}$,

$$
n^{-1 / 2} \widehat{\beta}_{Z} \stackrel{\mathcal{D}}{\longrightarrow} N\left(0, D_{Z}\right), n^{-1 / 2} \widehat{\beta}_{X} \stackrel{\mathcal{D}}{\longrightarrow} N\left(0, D_{X}\right) \text { e } n^{-1 / 2} \widehat{\beta}_{c} \stackrel{\mathcal{D}}{\longrightarrow} N\left(0, D_{c}\right) .
$$

(b) sob uma seqüência de alternativas $\beta^{(n)}, n^{1 / 2} \beta^{(n)} \rightarrow \Delta,\|\Delta\|>0$,

$$
\begin{aligned}
& n^{-1 / 2} \widehat{\beta}_{Z} \stackrel{\mathcal{D}}{\longrightarrow} N\left(\Delta, D_{Z}\right), n^{-1 / 2} \widehat{\beta}_{X} \stackrel{\mathcal{D}}{\longrightarrow} N\left(R \Delta, D_{X}\right) \text { e } n^{-1 / 2} \widehat{\beta}_{c} \stackrel{\mathcal{D}}{\longrightarrow} N\left(\Delta, D_{c}\right), \\
& \text { onde } \\
& D_{Z}=d \Sigma_{z}^{-1}, D_{X}=d\left(\Sigma_{z}+\Sigma_{u}\right)^{-1} \text {, e } D_{c}=d\left(I+\sigma_{z}^{-1} \Sigma_{u}\right) \Sigma_{z}^{-1}, \\
& \text { com } R=\left(\Sigma_{z}+\Sigma_{u}\right)^{-1} \Sigma_{z} \text { ed } d=\phi^{2} .
\end{aligned}
$$

Prova: A prova é análoga à da Proposição 3.5. Agora,

$$
n^{1 / 2} \widehat{\beta}_{Z} \stackrel{\mathcal{D}}{\longrightarrow} N\left(0, D_{Z}\right),
$$

onde $D_{Z}=E[-\dot{q}(\alpha)] \Sigma_{z}^{-1}$

$$
n^{1 / 2} \widehat{\beta}_{X} \stackrel{\mathcal{D}}{\longrightarrow} N\left(0, D_{X}\right)
$$

onde $D_{X}=\{E[-\dot{q}(\alpha)]\}^{-2} E\left[q^{2}(\alpha)\right]\left(\Sigma_{z}+\Sigma_{u}\right)^{-1}$, e

$$
n^{1 / 2} \widehat{\beta}_{c} \stackrel{\mathcal{D}}{\longrightarrow} N\left(0, D_{c}\right),
$$

onde $D_{c}=\left[\left\{E\left[-\frac{\partial U_{i}^{*}}{\partial \theta^{\prime}}\left(\theta_{0}\right)\right]\right\}^{-2} E\left[U_{i}^{*}\left(\theta_{0}\right) U_{i}^{*}\left(\theta_{0}\right)^{\prime}\right]\right]_{\beta \beta}$.

Como

$$
q(\alpha)=\frac{1}{\phi}\left[\exp \left(\frac{y-\alpha}{\phi}\right)-1\right]
$$


é fácil ver que

$$
E[-\dot{q}(\alpha)]=E\left[q^{2}(\alpha)\right]=\frac{1}{\phi^{2}} .
$$

Por outro lado,

$$
E\left[-\frac{\partial U_{i}^{*}\left(\theta_{0}\right)}{\partial \theta^{\prime}}\right]=\frac{1}{\phi^{2}}\left(\begin{array}{cc}
1 & \mu^{\prime} \\
\mu & \Sigma_{z}+\mu \mu^{\prime}
\end{array}\right)
$$

e

$$
E\left[U_{i}^{*}\left(\theta_{0}\right) U_{i}^{*}\left(\theta_{0}\right)^{\prime}\right]=\frac{1}{\phi^{2}}\left(\begin{array}{cc}
1 & \mu^{\prime} \\
\mu & \Sigma_{z}+\Sigma_{u}+\mu \mu^{\prime}
\end{array}\right) .
$$

Assim,

$$
\begin{aligned}
D_{Z} & =\phi^{2} \Sigma_{z}^{-1} \\
D_{X} & =\phi^{2}\left(\Sigma_{z}+\Sigma_{u}\right)^{-1} \\
\mathrm{e} \quad D_{c} & =\phi^{2}\left(I+\Sigma_{z}^{-1} \Sigma_{u}\right) \Sigma_{z}^{-1}
\end{aligned}
$$

e a proposição fica provada. 


\section{Considerações finais}

Para certos modelos lineares generalizados e distribuições do erro de medição, o método do escore corrigido é facilmente aplicado, produzindo estimadores consistentes e assintoticamente normais, sem fazer suposições sobre a distribuição das verdadeiras covariáveis $z$. No modelo funcional, as variáveis $z$ são constantes fixas e, portanto, os parâmetros desconhecidos incluem as $z$ 's. Com uma estrutura aditiva de erros de medição, uma estratégia seria maximizar a densidade conjunta dos dados observados com respeito a todos os parâmetros desconhecidos, incluindo $\left\{z_{i}\right\}_{i=1}^{n}$. Enquanto isto funciona para o modelo de regressão linear (Gleser, 1981), não é aplicável em geral para modelos mais complexos. Um enfoque alternativo é considerar o modelo estrutural e aplicar técnicas de verossimilhança. No entanto, essas técnicas precisam assumir um modelo para a distribuição de $z$, às vezes condicionada às covariáveis observadas. Alguns desses métodos produzem estimadores consistentes em casos especiais importantes, como regressão linear e modelos com média log-linear, mas os estimadores são "aproximadamente consistentes", em geral. Além disso, um problema difícil de resolver é a possível falta de robustez da inferência devido a uma especificação incorreta da distribuição das $z$ 's.

Um outro método que produz estimadores consistentes e assintoticamente normais, sem necessidade de que sejam feitas suposições sobre as verdadeiras covariáveis, é o método baseado no escore condicional (Stefanski e Carroll, 1987). Este método só pode ser aplicado em modelos lineares generalizados na forma canônica. Contudo, tem certas vantagens em termos de eficiência. 
Não é possível concluir que tipo de enfoque é mais apropriado em geral. A escolha dependerá do problema particular, da informação disponível e do interesse do pesquisador.

Por outro lado, a aplicação da técnica do escore corrigido é limitada àqueles modelos para os quais a existência da função escore corrigida possa ser garantida. Para isto, a suposição de normalidade sobre a distribuição do erro de medição é essencial. Contudo, mostramos que o uso de uma normal contaminada permite levar em conta possíveis "outliers" presentes nas covariáveis observadas. Alguns modelos importantes para os quais a função escore corrigida não existe, são o modelo de regressão logística e o modelo de riscos proporcionais de Cox, de grande importância em análise de sobrevivência. O uso de escores corrigidos aproximados e o estudo das propriedades dos estimadores para estes modelos é motivação para futuros estudos.

Um outro problema que merece particular atenção é como o uso de medições repetidas das verdadeiras covariáveis para a estimação da variância do erro de medição afeta as estimativas dos parâmetros. As Tabelas 5.4, 5.5 e 5.6 mostram uma redução no vício e no erro padrão dos estimadores quando duas medições repetidas das verdadeiras covariáveis são consideradas. Uma pergunta que surge é quantas medições repetidas seriam apropriadas. Liu e Liang (1992) estudam a eficiência do procedimento baseado em equações de estimação de Liang e Liu (1991) em termos do número de medições repetidas.

$\mathrm{Na}$ literatura, o estudo de testes de hipóteses para modelos com erros nas variáveis, tem se concentrado no problema de testar a hipótese de não associação entre uma covariável e uma variável resposta em modelos lineares generalizados. Tosteson e Tsiatis (1988) mostram que o teste escore "naive" e o teste escore eficiente são assintoticamente equivalentes quando $E_{x}[z]$ for função linear de $x$. Na Proposição 3.3 mostramos que, se além disso, considerarmos uma estrutura aditiva de erros de medição, tais estatísticas coincidem, para todo $n$. Por outro lado, as estatísticas do teste escore corrigido e do teste escore "naive" também coincidem, para todo $n$, quando assumimos uma estrutura aditiva de erros de 
medição, normalmente distribuídos. A comparação de testes de Wald é considerada para a família Tweedie de modelos com média log-linear e para o modelo de regressão linear normal, dado que, para esses modelos, sob a suposição de normalidade nas verdadeiras covariáveis, o vício assintótico do estimador "naive" pode ser obtido explicitamente. Mostramos que, quando uma única covariável é considerada, a eficiência assintótica relativa do teste de Wald "naive" com respeito ao teste quando $z$ é realmente observada é igual ao quadrado da correlação entre $x$ e $z$. O mesmo resultado é obtido por Lagakos (1988) e Tosteson e Tsiatis (1988) para outros tipos de testes. O teste de Wald "naive" e o teste de Wald baseado no estimador do escore corrigido são assintoticamente equivalentes. Um resultado análogo foi obtido por Stefanski e Carroll (1990) em relação ao teste de Wald "naive" e o teste baseado no estimador de Stefanski (1985). Portanto, observamos que o poder local do teste não é afetado quando no lugar de utilizar um estimador inconsistente do parâmetro $\beta$, utilizamos um estimador consistente como o estimador corrigido. Este fato pode ser devido ao fenômeno conhecido como "bias versus variance trade-off" (Carroll et al., 1995). Dado que reduzir o vício de um estimador resulta num aumento da variância, isto faz com que o poder local do teste de Wald baseado num estimador inconsistente (mas com variância menor) não seja afetado quando substituído por um estimador consistente (mas com variância maior).

Finalmente, notamos que os resultados dos Capítulos 2, 4 e 5 podem ser estendidos sem dificuldade ao caso em que existem covariáveis medidas sem erro. Com efeito, considere $z=\left(z_{1}^{\prime}, z_{2}^{\prime}\right)$, onde $z_{1}$ é observada exatamente, e no lugar de $z_{2}$ observamos $x_{2}=z_{2}+u$. As$\operatorname{sim}$, se o escore usual é dado por $U\left(\theta ; Z_{1}, Z_{2}, Y\right)=\sum_{i=1}^{n} U_{i}\left(\theta ; z_{1 i}, z_{2 i}, y_{i}\right)$, o escore corrigido $U^{*}$ tem a propriedade que $E\left[U^{*}\left(\theta ; Z_{1}, X_{2}, Y\right) \mid Z_{1}, Z_{2}, Y\right]=U\left(\theta ; Z_{1}, Z_{2}, Y\right)$. Com respeito aos resultados do Capítulo 3 , se $\beta=\left(\beta_{1}^{\prime}, \beta_{2}^{\prime}\right)^{\prime}$ e $\beta^{\prime} z=\beta_{1}^{\prime} z_{1}+\beta_{2}^{\prime} z_{2}$, é de interesse estudar o comportamento dos testes, quando testamos $H_{0}: \beta_{2}=0$ na presença do parâmetro "nuisance" $\left(\alpha, \beta_{1}^{\prime}\right)^{\prime}$. 


\section{Referências bibliográficas}

ANDERSEN, E.B. (1970). Asymptotic properties of conditional maximum likelihood estimators. Journal of the Royal Statistical Society, Series B, 32, 283-301.

ANDERSON, T.W (1984). An Introduction to Multivariate Statistical Analysis. 2.ed. New York: Wiley.

ARMSTRONG, B. (1985). Measurement error in generalized linear models. Communications in Statistics, Series B, 14, 529-544.

BARNETT, V.D. (1969). Simultaneous pairwise linear structural relationship. Biometrics, 25, 129-142.

BERKSON, J. (1950). Are there two regressions? Journal of the American Statistical Association, 45, 164-180.

CARROLL, R.J.; RUPPERT, D. and STEFANSKI, L.A. (1995). Measurement Error in Nonlinear Models. Chapman \& Hall, London.

CARROLL, R.J.; SPIEGELMAN, C.; LAN, K.K.; BAILEY, K.T. and ABBOTT, R.D. (1984). On errors-in-variables for binary regression models. Biometrika, 71, 19-26.

CARROLL, R.J. and STEFANSKI, L.A. (1990). Approximate quasilikelihood estimation in models with surrogate predictiors. Journal of the American Statistical Association, 85, 652-663.

CARTER, R. (1981). Restricted maximum likelihood estimation of bias and reliability in the comparison of several measuring methods. Biometrics, 37, 733-741.

COX, D.R. and HINKLEY, D.V. (1974). Theoretical Statistics. Chapman \& Hall, London.

CHENG, C.L. and VAN NESS, J.W. (1991). On the unreplicated ultrastructural model. Biometrika, 78, 442-445. 
CHENG, C.L. and VAN NESS, J.W. (1994). On estimating linear relationships when both variables are subject to errors. Journal of the Royal Statistical Society, Series B, 56, 167-183.

CRAMÉR, H. (1957). Mathematical Methods of Statistics. Princeton: Princeton University Press.

DEMPSTER, A.; LAIRD, N. and RUBIN, D.B. (1977). Maximum likelihood estimation from incomplet data via the EM algorithm (with discussion). Journal of the Royall Statistical Society, B, 39, 1-38.

EFRON, B. and MORRIS, C. (1973). Stein's estimation rule and its competitors. An empirical Bayes approach. Journal of the American Statistical Association, 68, $117-130$.

EFRON, B. and MORRIS, C. (1975). Data analysis using Stein's estimators and its generalizations. Journal of the American Statistical Association, 70, 311-319.

FOUTZ, R.V. (1977). On the unique consistent solution to the likelihood equations. Journal of the American Statistical Association, 72, 147-148.

FOUTZ, R.V. and SRIVASTAVA, R.C. (1977). The performance of the likelihood ratio test when the model is incorrect. The Annals of Statistics, 5, 1183-1194.

FULLER, W.A. (1987). Measurement Error Models. John Wiley \& Sons, New York.

FULLER, W.A. (1995). Estimation in the presence of measurement error. International Statistical Review, 63, 121-147.

GALEA-ROJAS, M. (1995). Calibração Comparativa Estrutural e Funcional. Tese, IME-USP.

GLESER, L.J. (1981). Estimation in a multivariate errors in variables regression model: large sample results. Annals of Statistics, 9, 24-44.

GLESER, L.J. (1983). Functional, structural and ultrastructural errors-in-variables model. In Proc. Bus. Econ. Statist. Sect., Am. Statist. Assoc., 57-66.

GLESER, L.J. (1985). A note on G.R. Dolby's unreplicated ultrastructural model. Biometrika, 72, 117-124. 
GLESER, L.J. (1990). Improvements of the naive approach to estimation in nonlinear errors-in-variables regression models. In Statistical Analysis of Measurement Error Models and Application. P.J. Brown and W.A. Fuller, editors. American Mathematical Society, Providence.

GRIFFITHS, P. and HILL, I.D. (1985). Applied Statistics Algorithms. London: Horwood.

HOADLEY, B. (1971). Asymptotic properties of maximum likelihood estimators for the independent not identically distributed case. Annals of Mathematical Statistics, 42, 1977-1991.

HUBER, P.J. (1981). Robust Statistics. New York: Willey.

JAECH, J.L. (1985). Statistical Analysis of Measurement Errors. Exxon Monographs. New York: John Wiley.

JØRGENSEN, B. (1987). Exponential dispersion models (with discussion). Journal of the Royal Statistical Society, Series B, 127-162.

KANNEL, W.B.; NEATON, J.D.; WENTWORTH, D.; THOMAS, H.E.; STAMBER, J.; HULLEY, S.B. and KJELSBERG, M.O. (1986). Overall and coronary heart disease mortality rates in relation to major risk factors in 325,348 men screened for MRFIT. American Heart Journal, 112, 825-836.

KENDALL, M.G. and STUART, A. (1979). The Advanced Theory of Statistics, 2. 4.ed. New York: Hafner.

KIMURA, D.K. (1992). Functional comparative calibration using an EM algorithm. Biometrics, 48, 1263-1271.

KOLMOGOROV, A.N. and FOMIN, S.V. (1975). Elementos de la Teoría de Funciones y del Análisis Funcional. 2.ed. Moscu: Mir.

LAGAKOS, S.W. (1988). Effects of mismodeling and mismeasuring explanatory variables on tests of their association with a response variable. Statistics in Medicine, 7, 257-274.

LIANG, K.Y. and LIU, X.H. (1991). Estimating equations in generalized linear models with measurement error. In Estimating Functions, V.P. Godambe, editor. Clarendon Press, Oxford. 
LINDLEY, D.V. (1947). Regression lines and the linear functional relationship. Suppl. Journal of Royal Statistical Society, 9, 218-244.

LIU, X. and LIANG, K.Y. (1992). Efficacy of repeated measures in regression models with measurement error. Biometrics, 48, 645-654.

MAK, T.K. (1982). Estimation in the presence of incidental parameters. The Canadian Journal of Statistics, 10, 121-132.

MARAZZI, A. (1980). ROBETH, a subroutine library for robust statistical procedures. COMPSTAT 1980, Proceedings in Computational Statistics, Physica, Vienna.

McCULLAGH, P. and NELDER, J.A. (1989). Generalized Linear Models. 2.ed. London: Chapman \& Hall.

NAKAMURA, T. (1990). Corrected score functions for error-in-variables models: methodology and application to generalized linear models. Biometrika, 77, 127-137.

NEYMAN, J. and SCOTT, E. (1948). Consistent estimates based on partially consistent observations. Econometrica, 16, 1-32.

NORDEN, R.H. (1973). A survey of maximum likelihood estimation, II. Rev. Inst. Internat. Statist., 41, 39-58.

OKAJIMA, S.; MINE, M. and NAKAMURA, T. (1985). Mortality of registered A-bomb survivors in Nagasaki, Japan, 1970-1984. Radiation Research, 103, 419-431.

PATEFIELD, W.M. (1977). On the information matrix in the linear functional relationship problem. Applied Statistics, 26, 69-70.

PATEFIELD, W.M. (1978). The unreplicated ultrastructural relation: large sample properties. Biometrika, 65, 535-540.

PHILIPPOU, A.N. and ROUSSAS, G.G. (1975). Asymptotic normality of the maximum likelihood estimate in the independent not identically distributed case. Ann. Inst. Statist. Math., 27, 45-55.

PRENTICE, R.L. (1982). Covariate measurement errors and parameter estimation in a failure time regression model. Biometrika, 69, 331-342.

RAO, C.R. (1973). Linear Statistical Inference and its Applications. 2.ed. New York: Wiley. 
RAO, J.N.K. and SCOTT, A.J. (1981). The analysis of categorical data from complex sample surveys: chi-squared tests for goodness of fit and independence in two-way tables. Journal of the American Statistical Association, 76, 221-230.

ROTNITZKY, A. and JEWELL, J. (1990). Hypothesis testing of regression semiparametric generalized linear models for clustered correlated data. Biometrika, 77, 485-497.

ROYALL, R.M. (1986). Model robust inference using maximum likelihood estimators. International Statistical Review, 54, 221-226.

RUDIN, W. (1964). Principles of Mathematical Analysis. New York: McGraw Hill.

SCHAFER, D. (1987). Covariate measurement error in generalized linear models. Biometrika, 74, 385-391.

SEN, P.K. and SINGER, J.M. (1993). Large Sample Methods in Statistics: An Introduction with Applications. New York, Chapman \& Hall, Inc.

SERFLING, R.J. (1980). Approximation Theorems of Mathematical Statistics. John Wiley \& Sons, New York.

STEFANSKI, L.A. (1985). The effects of measurement error on parameter estimation. Biometrika, 78, 583-592.

STEFANSKI, L.A. (1989). Unbiased estimation of a nonlinear function of a normal mean with application to measurement error models. Communications in Statistics, Series A, 18, 4335-4358.

STEFANSKI, L.A. and CARROLL, R.J. (1985). Covariate measurement error in logistic regression. Annals of Statistics, 13, 1335-1351.

STEFANSKI, L.A. and CARROLL, R.J. (1987). Conditional scores and optimal scores in generalized linear measurement error models. Biometrika, 74, 703-716.

STEFANSKI, L.A. and CARROLL, R.J. (1990). Score tests in generalized linear measurement error models. Journal of the Royal Statistical Society, Series B, 52, 345-359.

THEOBALD, C.M. and MALlinSON, J.R. (1978). Comparative calibration, linear structural relationship and congeneric measurement. Biometrics, 34, 39-45. 
TOSTESON, T. and TSIATIS, A. (1988). The asymptotic relative efficiency of score tests in a generalized linear model with surrogate covariates. Biometrika, 75, 507514.

TUKEY, J.W. (1960). A survey of sampling from contamined distributions. In Contributions to Probability and Statistics. Olkin, Ed., Stanford University Press, Stanford, Calif.

WEISS, L. (1971). Asymptotic properties of maximum likelihood estimators in some nostandar cases. Journal of the American Statistical Association, 66, 345-350.

WEISS, L. (1973). Asymptotic properties of maximum likelihood estimators in some nonstandar cases, II. Journal of the American Statistical Association, 68, 428-430.

WEISS, L. (1975). The asymptotic distribution of the likelihood ratio in some nonstandar cases. Journal of the American Statistical Association, 70, 204-208.

WHITTEMORE, A.S. (1989). Errors in variables regression using Stein estimates. American Statistician, 43, 226-228.

WHITTEMORE, A.S. and KELLER, J.B. (1988). Approximations for regression with covariate measurement error. Journal of the American Statistical Association, 83, 1057-1066.

WOLTER, K.M. and FULLER, W.A. (1982). Esîimation of nonlinear errors-in-variables models. Annals of Statistics, 10, 539-548. 